\title{
SPECULATIVE COMPUTING: \\ INSTRUMENTS FOR INTERPRETIVE SCHOLARSHIP
}

\author{
Bethany Paige Nowviskie \\ Charlottesville, Virginia
}

\author{
B.A. University of Virginia, 1995 \\ MA.Ed. Wake Forest University, 1996
}

\begin{abstract}
A Dissertation Presented to the Graduate Faculty
of the University of Virginia in Candidacy for the Degree of

Doctor of Philosophy

Department of English

University of Virginia

May 2004
\end{abstract}

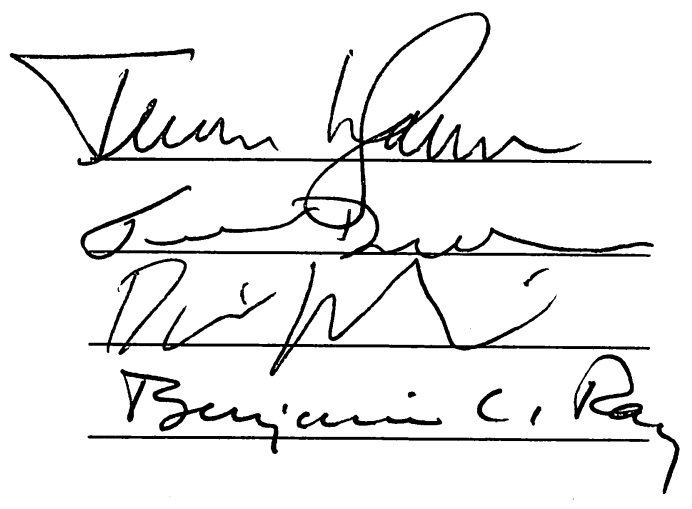




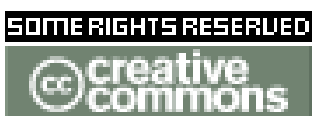

This work is licensed under the Creative Commons Attribution-NoDerivs License.

To view a copy of this license, visit http://creativecommons.org/licenses/by-nd/1.0/ or send a letter to Creative Commons, 559 Nathan Abbott Way, Stanford, California 94305, USA. 


\begin{abstract}
Like many modern humanities computing projects, Ramon Llull's Ars Magna, a system of inscripted, manipulable wheels dating to the thirteenth century, asserts that interpretation can be aided by mechanism without being generated mathematically or mechanically. That this assertion is sometimes lost on the larger academic community is not simply a failure of the devices scholar-technologists produce (although, as the work outlined here seeks to demonstrate, we could do a better job of anticipating and incorporating patently interpretive or subjective forms of interaction on the part of our users into the systems we create for them). Instead, it betrays our failure to articulate the humanistic and hermeneutic value of algorithmic work to a lay audience.
\end{abstract}

This dissertation uses Llull's Ars Magna to introduce the relationships of algorithm, ars combinatoria, aesthetic provocation, diagrammatic reasoning, and ludic practice to the work of humanities scholarship and then presents two major case studies in the design of digital instruments and environments that open themselves to performance and intervention on the part of interpretive agents. The first is the Temporal Modelling PlaySpace, a composition tool for sketching subjective and inflected timelines that (like temporal relations in humanities data generally) are not necessarily unidirectional, homogenous, or continuous. Temporal Modelling's innovation lies in its extraction for re-purposing of well-formed XML from users' intuitively-designed and even 
deliberately ambiguous diagrammatic models. The second case study deals with computational and interface or visualization strategies for turning problems in representing subjectivity and deixis into opportunities for critical engagement in the Ivanhoe Game, a ludic subset of the larger IVANHOE project, an interpretive roleplaying environment conceived by Jerome McGann and Johanna Drucker.

Both of these projects stem from work in progress at the University of Virginia's Speculative Computing Laboratory. The goals and methods of SpecLab are demonstrated (and tested in performance) in a trio of creative design exercises or “imaginary solutions” which make use of ideas developed in chapters on Llull, Temporal Modelling, and the Ivanhoe Game — and "speculative computing" is introduced as a new paradigm for exploratory digital work in the humanities. 


\section{Preconditions}

"Freedom is compatible with necessity."

— from the journals of Gerard Manley Hopkins

Essential Oils - are wrung -

The Attar from the Rose

Be not expressed by Suns - alone -

It is the gift of Screws -

Emily Dickinson (675, ca.1863)

"Theories thus become instruments, not answers to enigmas,

in which we can rest. We don't lie back upon them, we move forward, and, on occasion, make nature over again by their aid. Pragmatism unstiffens all our theories, limbers them up and sets each one at work."

— William James, "What is Pragmatism?” (1904) 


\section{TABLE OF CONTENTS}

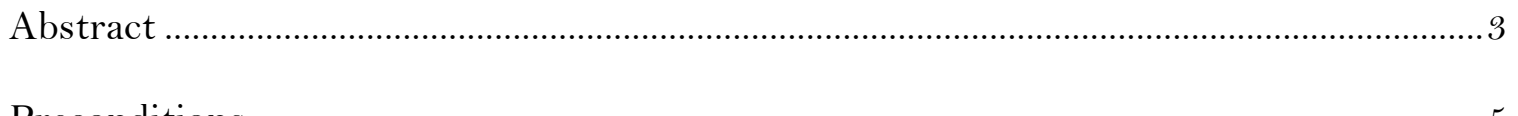

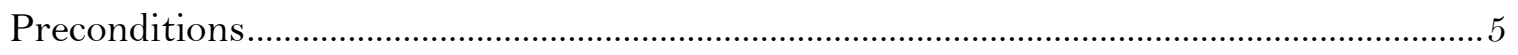

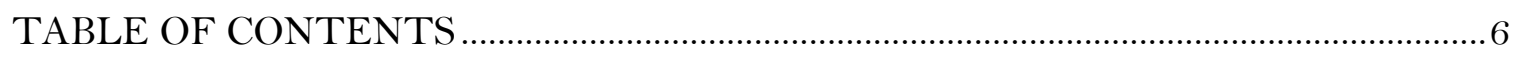

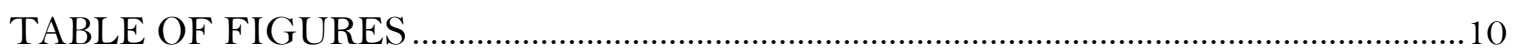

I. Mechanism and Interpretation in Humanities Computing............................................12

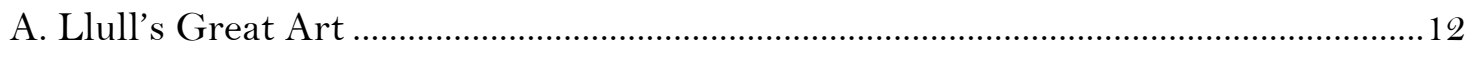

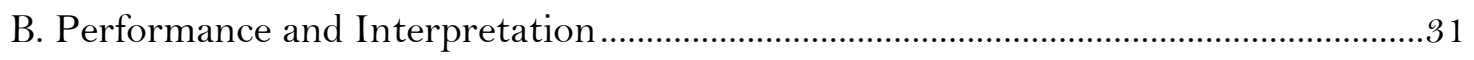

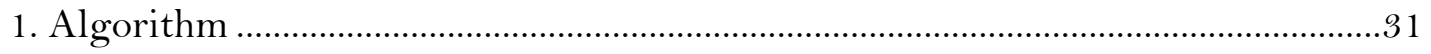

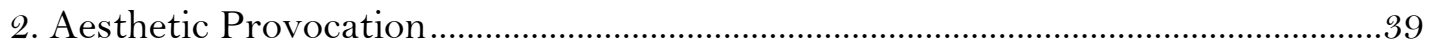

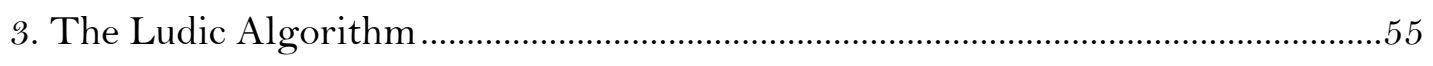

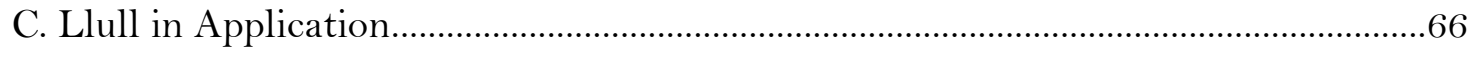

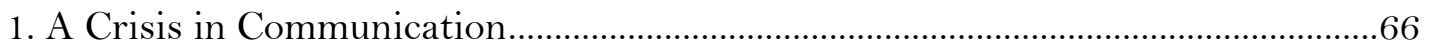

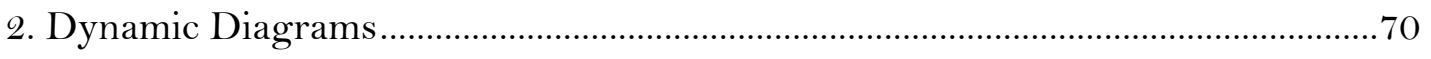

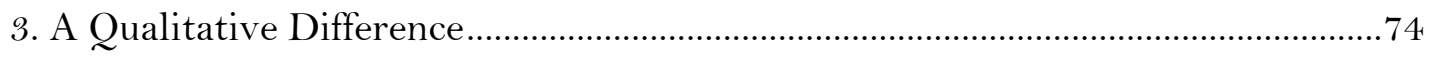

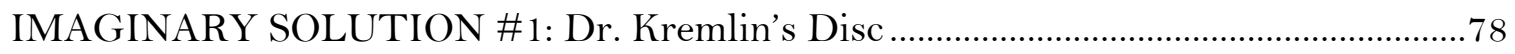




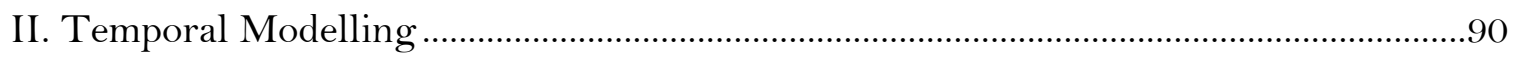

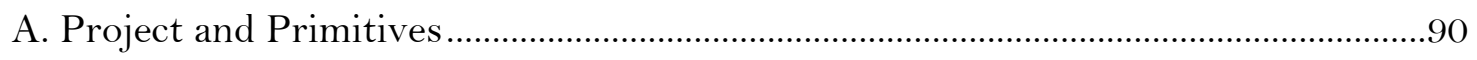

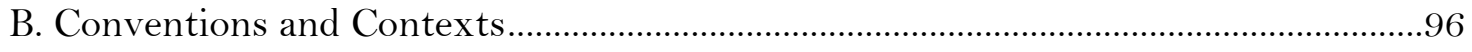

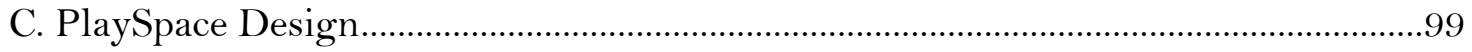

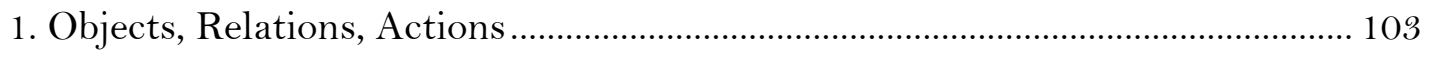

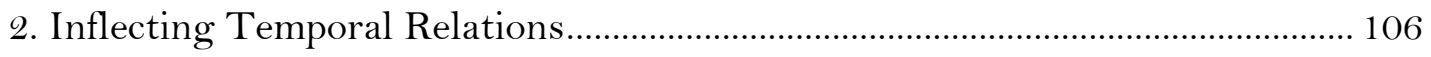

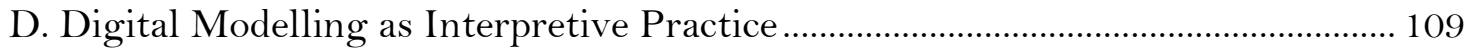

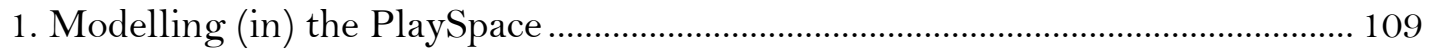

2. Diagrammatic Reasoning.................................................................................... 114

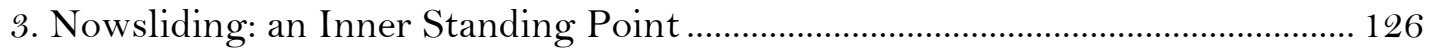

E. Directions: A Cognitive Science Approach.................................................................. 135

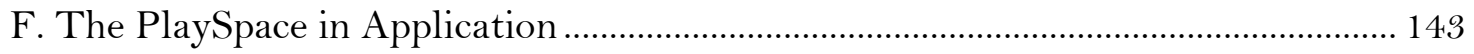

IMAGINARY SOLUTION \#2: Temporal Modelling at Sea …….................................... 151

III. Subjectivity in the Ivanhoe Game: Visual and Computational Strategies ............... 163

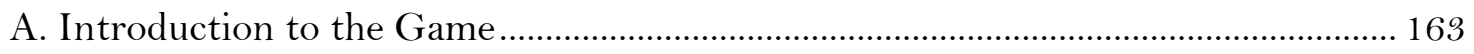

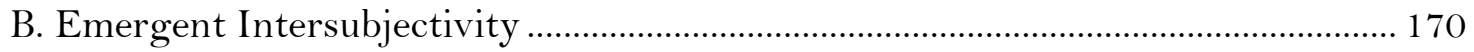

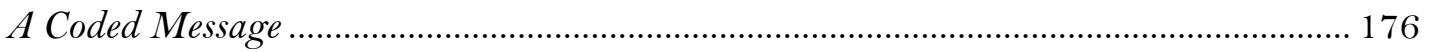

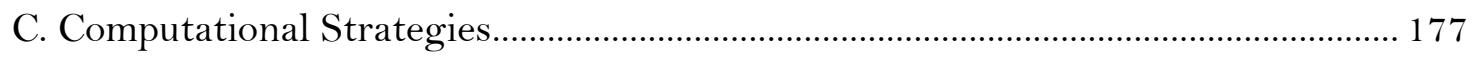

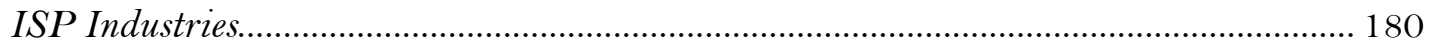




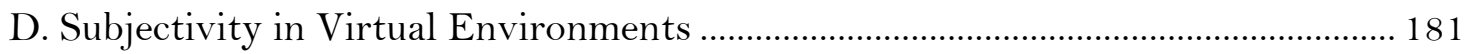

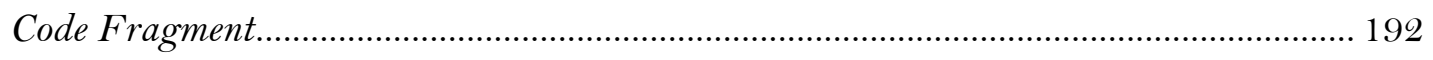

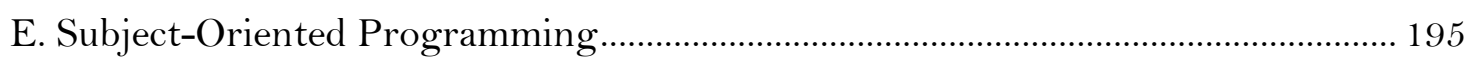

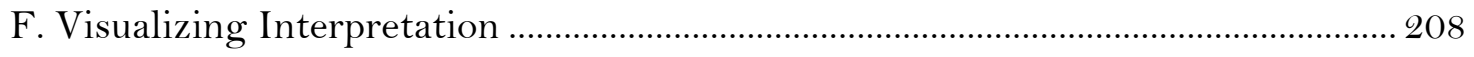

Bring Out Number Weight and Measure in a Year of Dearth...................................... 214

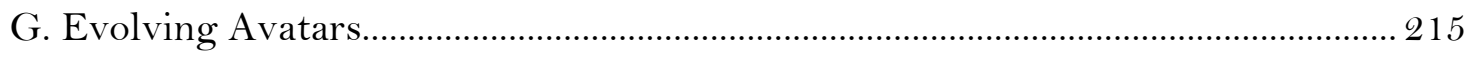

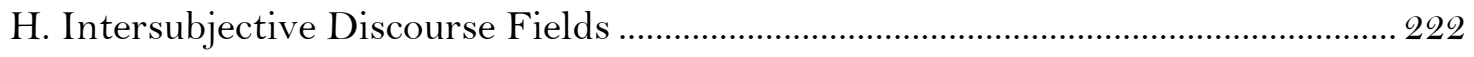

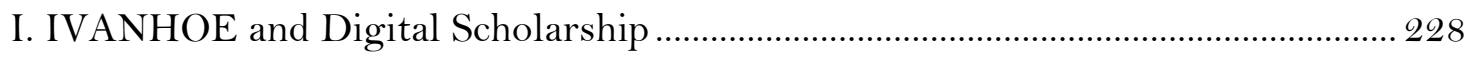

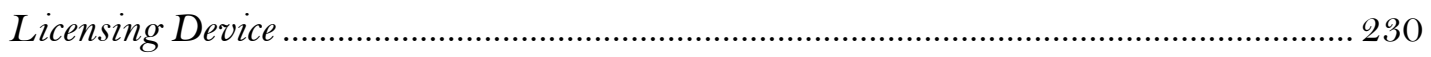

IMAGINARY SOLUTION \#3: The Somehow May Be Thishow ……...........................222

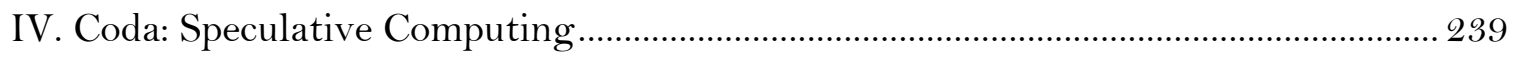

Appendix A. "Temporal Modelling: Conceptualization and Visualization of Temporal

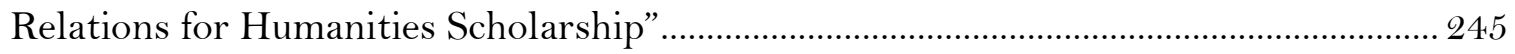

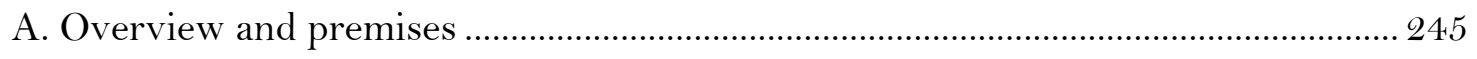

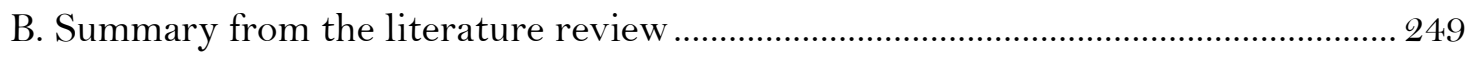

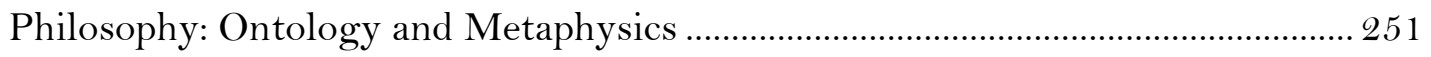

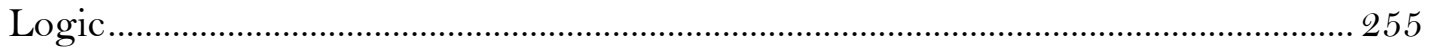

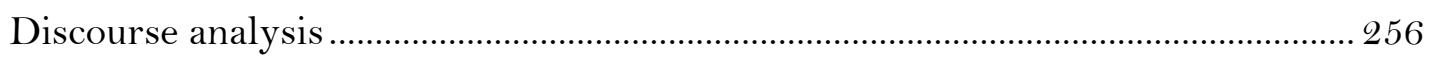

C. Graphic forms for representing time and temporality .............................................26 


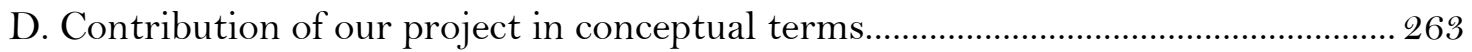

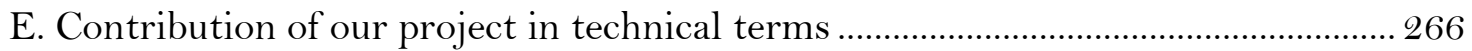

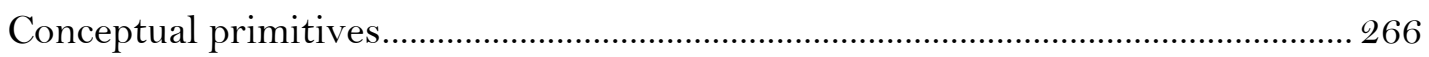

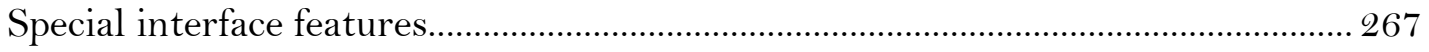

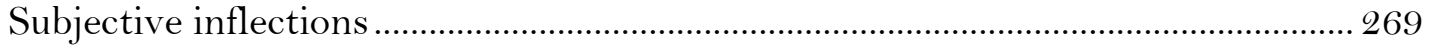

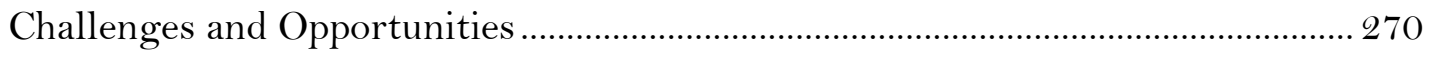

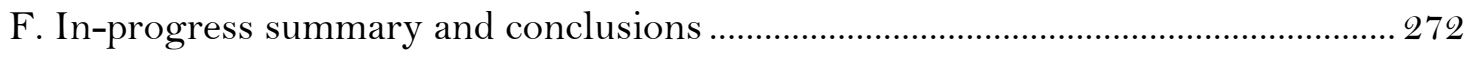

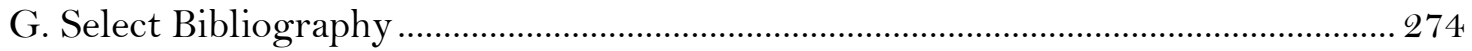

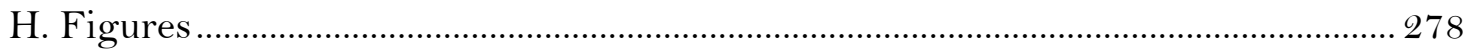

Appendix B. Original Temporal Modelling Software Proposal......................................... 283

Appendix C. Rossetti Archive Redesign Proposals..............................................................286

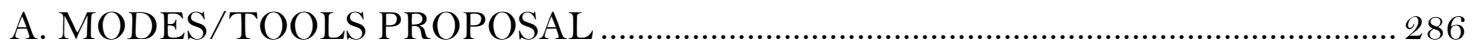

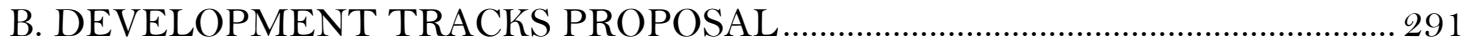

1. Redesign Job One: Supporting Pedagogy and the Scholarly Community ........ 291

2. Speculative Work: Showcasing Toolsets for Interpretive Application...............295

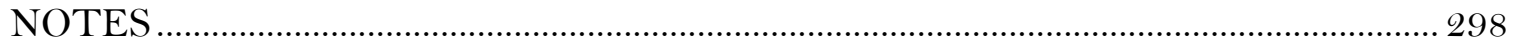

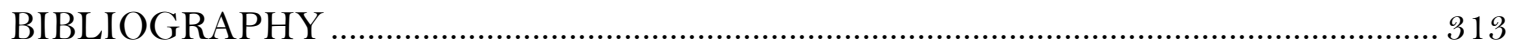




\section{TABLE OF FIGURES}

\section{CHAPTER I:}

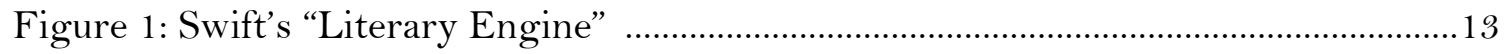

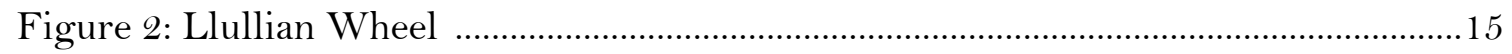

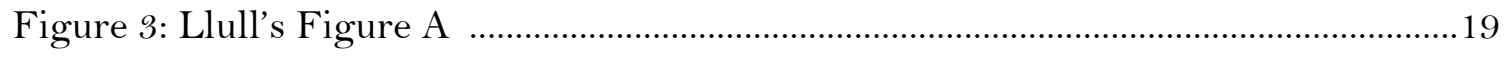

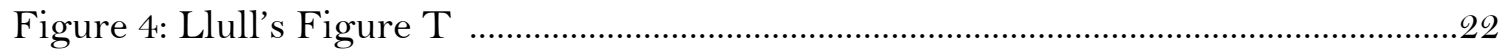

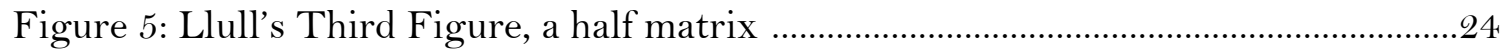

Figure 6: Llull's Fourth Figure, inter-rotating wheels ...................................................27

Figure 7a: Simple Algorithmic Deformation of Rossetti’s “Blessed Damozel” ...............64

Figure 7b: Intuitive, Computer-Assisted Deformation ......................................................65

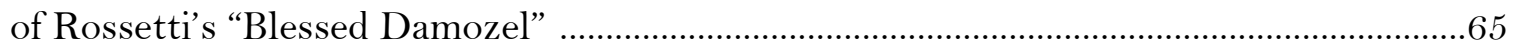

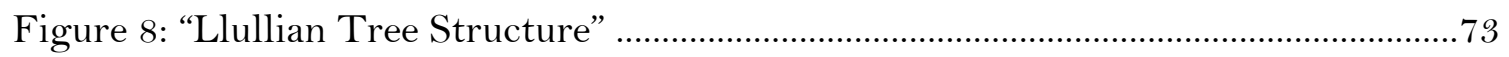

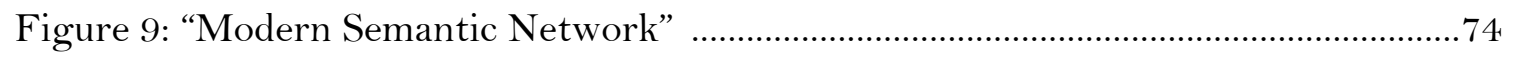

\section{CHAPTER II:}

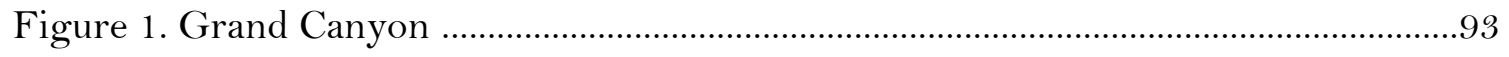

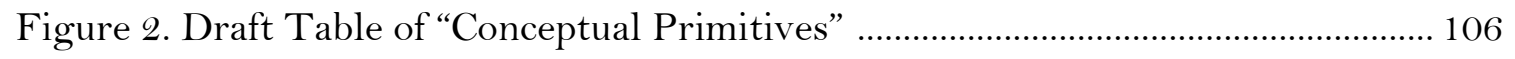

Figures 3ab: PlaySpace View and Corresponding Data Model …….............................. 112

Figures 4abc: Catastrophic Nowslider Screenshots and Descriptions ........................... 130 
Figure 5: Continuous Nowslider (a cut-your-own flip book) ..... 134

Figures 6abcd: Temporal Modelling PlaySpace in Application .....

\section{CHAPTER III:}

Figure 1: GraphVis Diagram of Turn of the Screw Gameplay, Andrea Laue 165

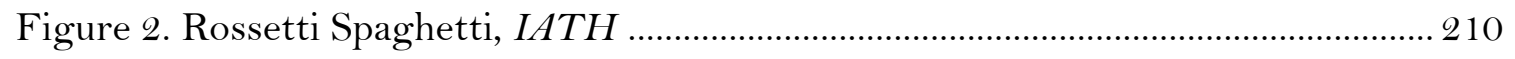

Figure 3. Evolving Avatars 217 


\section{Mechanism and Interpretation in Humanities Computing}

\section{A. Llull's Great Art}

Swift's Gulliver, on the aerial leg of his Travels, finds himself in the lofty scholastic community of Laputa, where he encounters a professor with a strange device. The mechanism consists of a series of rotating blocks on which are inscribed words in the Laputian language and which, in use, resemble nothing so much as a mystical foosball table (Figure 1). A few vigorous turns of the crank (for which the professor employs a team of undergraduates) produce what Robert De Beaugrande warns against as a "combinatoric explosion" of information: words combine randomly to produce sense and nonsense, the finest fragments of which are diligently recorded as the "wisdom" of Laputa (De Beaugrande). In this manner, Swift tells us, "the most ignorant person at a reasonable charge, and with a little bodily labour, may write books in philosophy, poetry, politics, law, mathematics, and theology, without the least assistance from genius or study" (Swift, Part III, Ch. 5).

The Laputian device, a "Project for improving speculative Knowledge by practical and mechanical means" (III. Ch.5), and Swift's unflattering description of the professor who invented it, are sometimes thought to satirize Leibniz, whose 1666 Dissertatio de Arte Combinatoria made far-reaching claims for the ability of mathematical and mechanical languages to generate wisdom and solve conflict. Leibniz went so far as to suggest that, 
in the future, every misunderstanding or disagreement "should be nothing more than a miscalculation... easily corrected.” Disputing philosophers could take up their abaci and settle even delicate theological arguments mechanically, saying "Calculemus!" "Let us compute!” (Leibniz).

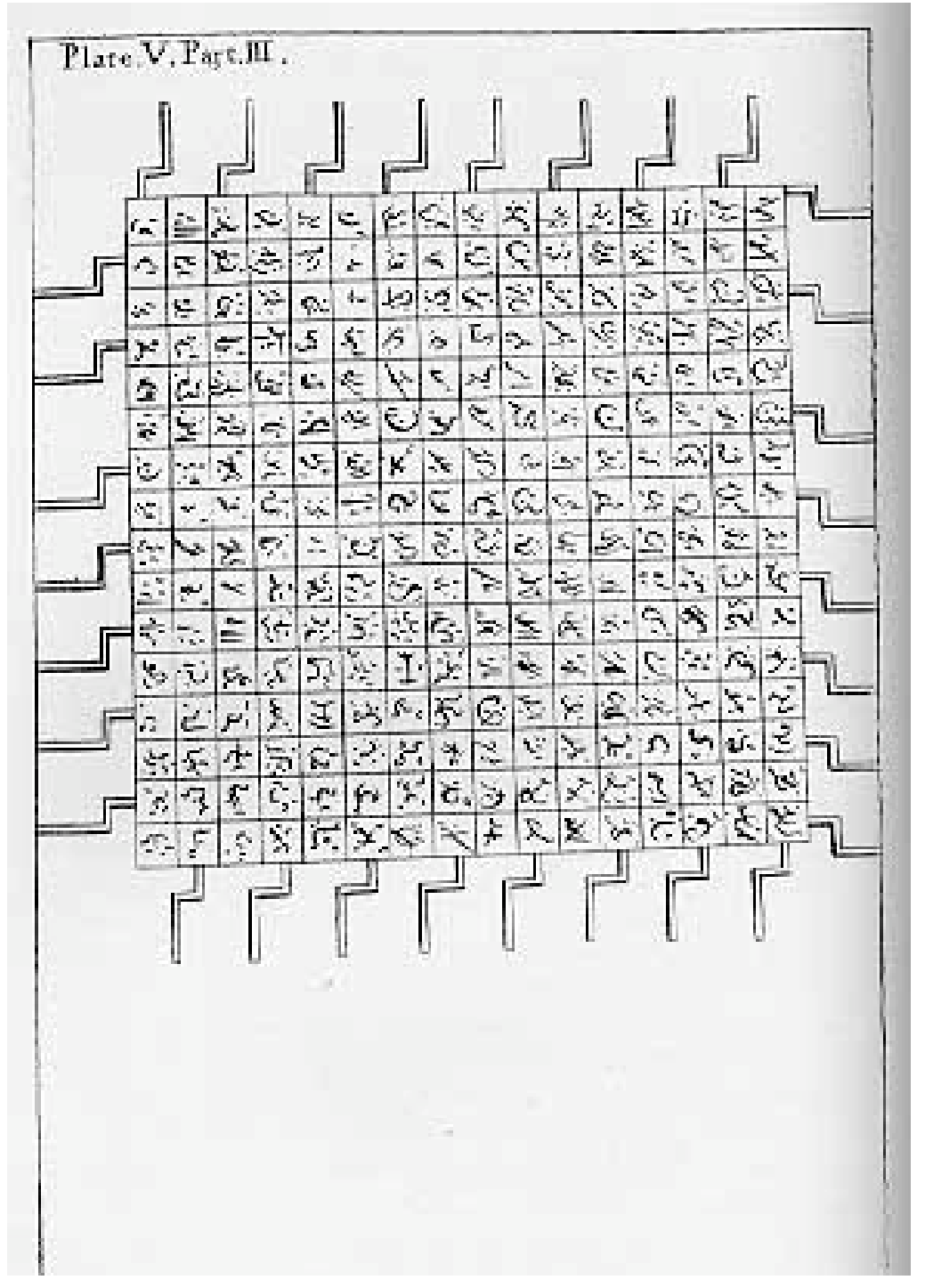

[ Figure 1: Swift's “Literary Engine”] 
In fact, a better-supported candidate for Swift's vitriol is Leibniz's acknowledged predecessor in the combinatoric arts, a colorful medieval polymath and sometime poet, rake, and martyr named Raimundus Lullus, or Ramon Llull (ca. 1232-1316). Lull's chief invention was a so-called Ars Magna of encoded, inter-rotating wheels developed in the latter decades of the thirteenth century and articulated in a treatise called the Ars Generalis Ultima. Its purpose was at once generative, analytical, and interpretive, and while its primary subject matter was theological, Llull was careful to demonstrate the applicability of the Ars Magna to broader philosophical and practical problems of the day. In other words, Llull's wheels constituted a user-extensible mechanical aid to hermeneutics and interpretive problem-solving (Figure 2). Properly understood, Llull and his Great Art can take their place, not in the soaring halls of Laputian "speculators" and pseudo-scientists, but among a cadre of humanists with fresh ideas about the relation of mechanism to interpretation.

A review and description of Llull's tool, with attention to its structure and function and to past misunderstandings as to its purpose, will help situate some instrumental design issues that seem novel in humanities computing today. Among these are problems involved in establishing scholarly primitives and developing the rules or algorithms by which they can be manipulated in creative and revelatory ways. We will also examine the relationship between algorithmic and combinatorial methods and subjective, interpretive practice in the humanities, and present preliminary results from the 
development of two performative instruments or environments that share in Llull's generative, emergent model of mechanism design.

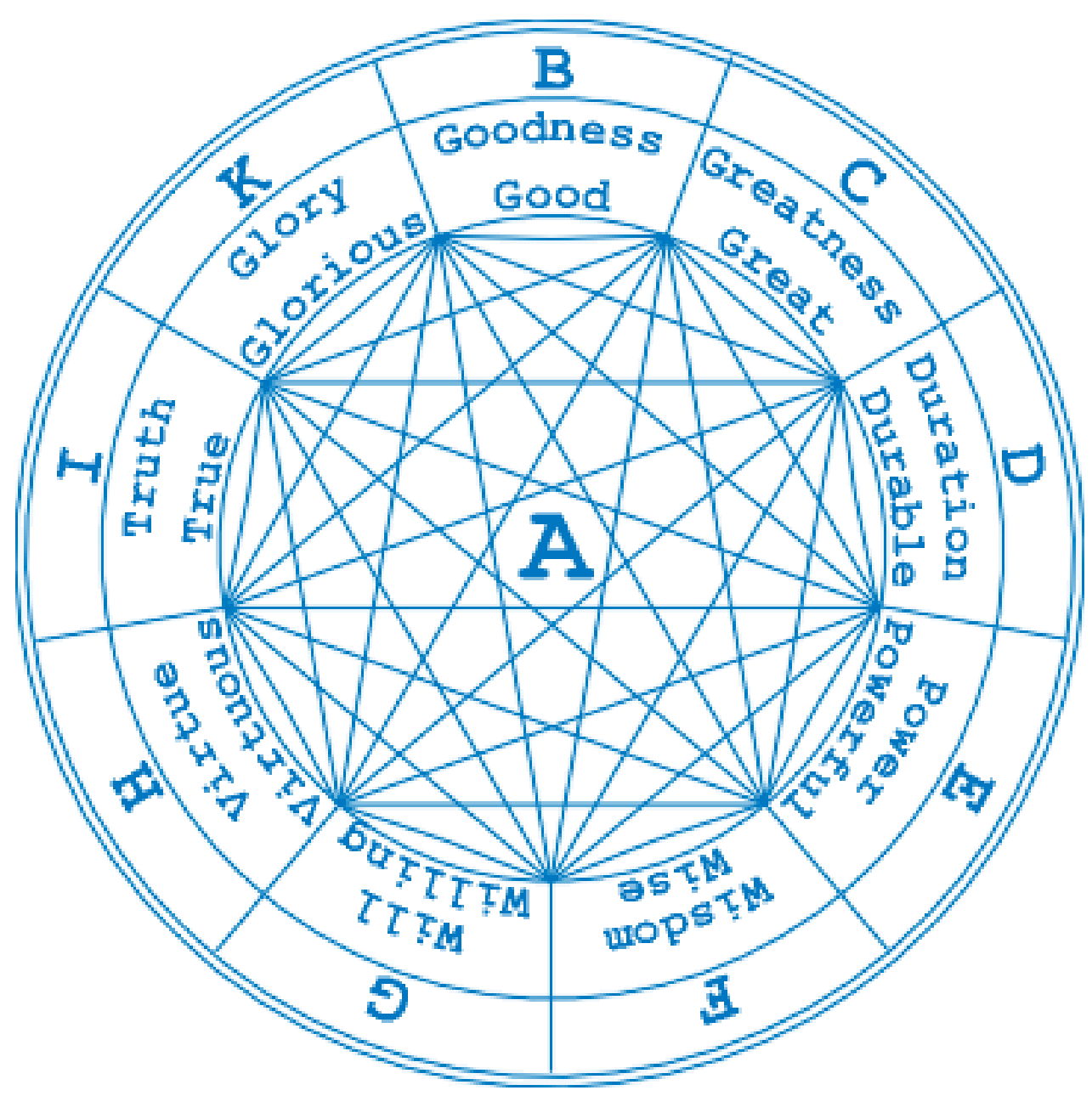

[ Figure 2: Llullian Wheel ]

Llull's intriguing mechanism is recognized as a precursor both to computer science in its emphasis on a mechanical calculus — and to the philosophy of language, in its use 
of symbols and semantic fields (Sales). However, after early widespread popularity in the universities of Renaissance Europe, it met with sharp criticism. ${ }^{1}$ Rabelais' Gargantua warns Pantagruel against Llullism in the same breath as "divinatory astrology;" it is only so much "fraud and vanity" (qtd. in Bonner 87). And Francis Bacon describes the Ars Magna as "a method of imposture... being nothing but a mass and heap of the terms of all arts, to the end that they who are ready with the terms may be thought to understand the arts themselves." Such collections, Bacon observes, "are like a fripper's or broker's shop, that has the ends of everything, but nothing of worth" (Bacon, VI.2).

Contemporary critics, too, deride Llull. Even Martin Gardner, whose 1950s study of Logic Machines and Diagrams views Llull as foundational to the history of visual and mechanical thinking — Llull is Chapter One! — suggests that the best uses for his once-influential combinatoric system are (in Gardner's words) "frivolous": for example, to generate possible names for a baby, to work anagram puzzles, or to compare and combine colors for application in design and interior decorating (Gardner 19).

Any more sophisticated or intellectually-laden use for Llull's device is, Gardner thinks, wholly inappropriate. The spinning wheels, when applied to humanistic subject matter lacking in native "analytic structure" and for which there is "not even agreement on what to regard as the most primitive, 'self-evident' principles," generate only circular proofs. "It was Lull's particular distinction," Gardner writes, "to base this type of 
reasoning on such an artificial, mechanical technique that it amounted virtually to a satire of scholasticism, a sort of hilarious caricature of medieval argumentation” (18). And it's true that examples of Llull's own symbolic and mechanically generated output read like something out of Swift's Laputa. Here is a characteristic excerpt from Chapter 335 of The Book of Contemplation, a precursor to the Ars Magna, in which Llull also asks alphabetic symbols like those inscribed on his wheels to stand in quasi-logical proofs for qualities of the Divine:

If in Thy three properties there were no difference... the demonstration would give the $\mathrm{D}$ to the $\mathrm{H}$ of the $\mathrm{A}$ with the $\mathrm{F}$ and the $\mathrm{G}$ as it does with the $\mathrm{E}$, and yet the $\mathrm{K}$ would not give significance to the $\mathrm{H}$ of any defect in the $\mathrm{F}$ or the $\mathrm{G}$; but since diversity is shown in the demonstration that the $\mathrm{D}$ makes of the $\mathrm{E}$ and the $\mathrm{F}$ and the $\mathrm{G}$ with the $\mathrm{I}$ and the $\mathrm{K}$, therefore the $\mathrm{H}$ has certain scientific knowledge of Thy holy and glorious Trinity. (Llull, Book of Contemplation)

Llull does himself no favors (to the modern mind) by using phrases like "certain scientific knowledge" of the Holy Trinity. However, it's not a far stretch for a computing humanist to become an unabashed fan of Ramon Llull. We may not wish to go so far (like his German proponents Peter Bexte and Werner Künzel) as to claim that Llull was “der erste Hacker in den himmlischen Datenbanken” (Bexte \& Künzel, intro), but it seems clear that the most scathing criticisms of the Ars Magna stem from a fundamental misunderstanding of the uses to which Llull meant his device to be put. 
Künzel is right, in The Birth of the Machine, to describe Llull's system of interlocking, inter-rotating wheels as an ancestor of the Turing Machine, a "logic machine, producing results, statements - output of data in general — by a clearly defined mechanical algorithm” (Künzel). However, we would be wrong to assume, with Bacon and Gardner, that the statements generated by this algorithm (that is, by the proscribed procedure of spinning wheels and diagramming results) are to be interpreted as truth. In fact, the linguistic combinations that Llull's wheels produce are only meant to be interpreted. That is, Llull invented a device for putting new ideas into the world out of the fragments of old ideas and constraining rulesets, but left the (inherently subjective) evaluation and explication of these emergent concepts up to a human user - a person explicitly figured in his writing as an "artista." Llull's machine generates "truthful” formulations equally with falsehood, and makes no claim about or evaluation of its own output: "naturally, only the artist using the machine is able to decide which statement is true and which is false. The machine independently produces both: the universe of truth and the universe of the false, step by step.” (Künzel). 


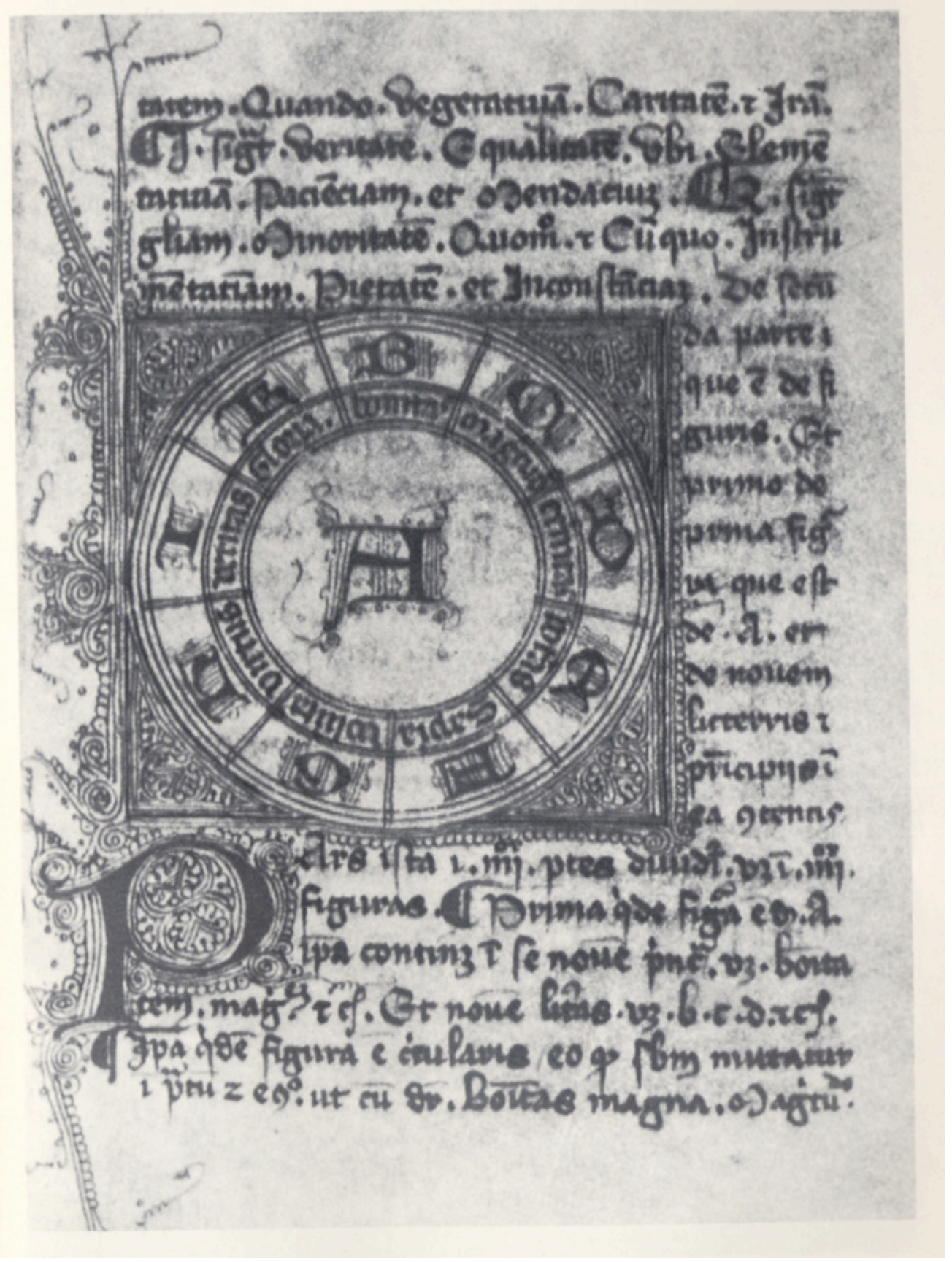

[ Figure 3: Llull's Figure A ] 
In building the Ars Magna, Llull began by establishing a manipulable alphabet of discrete, primary concepts or primitives on which his algorithmic and mechanical procedures could operate. The most commonly accepted (and least complex) version of this Art associates nine letters of the Latin alphabet, B through $\mathrm{K}$, with fundamental aspects of divinity: goodness, greatness, eternity or duration, power, wisdom, will, virtue, truth, and glory (Llull, Ars Brevis). The letter A stands in for the Divine, and is placed at the center of a circular diagram (Figure 3), which in itself becomes a hypothetical definition of God. ${ }^{2}$ When lines are drawn to connect each of the nine letter-coded aspects (showing in binaries, for example, that God's goodness is great [BC], God's virtue lies in truth [HI], etc.), Llull expresses the basic relational character not only of divinity, but of his very notion of an ars combinatoria. Combinatoric elements are not simply re-ordered, as with Swift's Laputian machine; here they are placed for careful consideration in conjunction.

The resultant graph — which, as we will later see, Llull considered to be dynamic rather than static - forms the most simple interpretive tool of the Ars Magna. The tool is properly thought of as interpretive rather than explicatory, because the conjoined components of the definition of God expressed in it were not meant to be flatly accepted by an audience, but rather contemplated, analyzed, and above all compared with their implied opposite, the qualities of fallen mankind. The rich rhetorical expression in these combinations comes into being through the user's own faculties of comparison and analogy as we see that, for example, the power (letter E) of human rulers - unlike that 
of the defined divinity — is not always commensurate with their wisdom (letter F).

As a next step, Llull's binary relationships are complicated by the application of a separate assemblage of meanings to his established alphabet. The letters B through K are secondarily associated with a "set of relational principles related among themselves in three groups of three”: difference, concordance, and contrariety; beginning, middle, and end; and majority, equality, and minority (Bonner, "What Was Llull Up To?”). These principles are laid out in another circle, Llull's Figure T, in which their tripartite associations are expressed through triangular graphs (Figure 4). "Beginning," "middle," and "end," for example, become the points of a triangle set within concentric circles - a pattern which is, in fact, the material expression of Llull's abstract, interpretive ruleset and which shows the domains to which these notions may be applied. The concept of an ending, for instance, may be interpreted here as it relates to labelled notions of privation, termination, or perfection. 


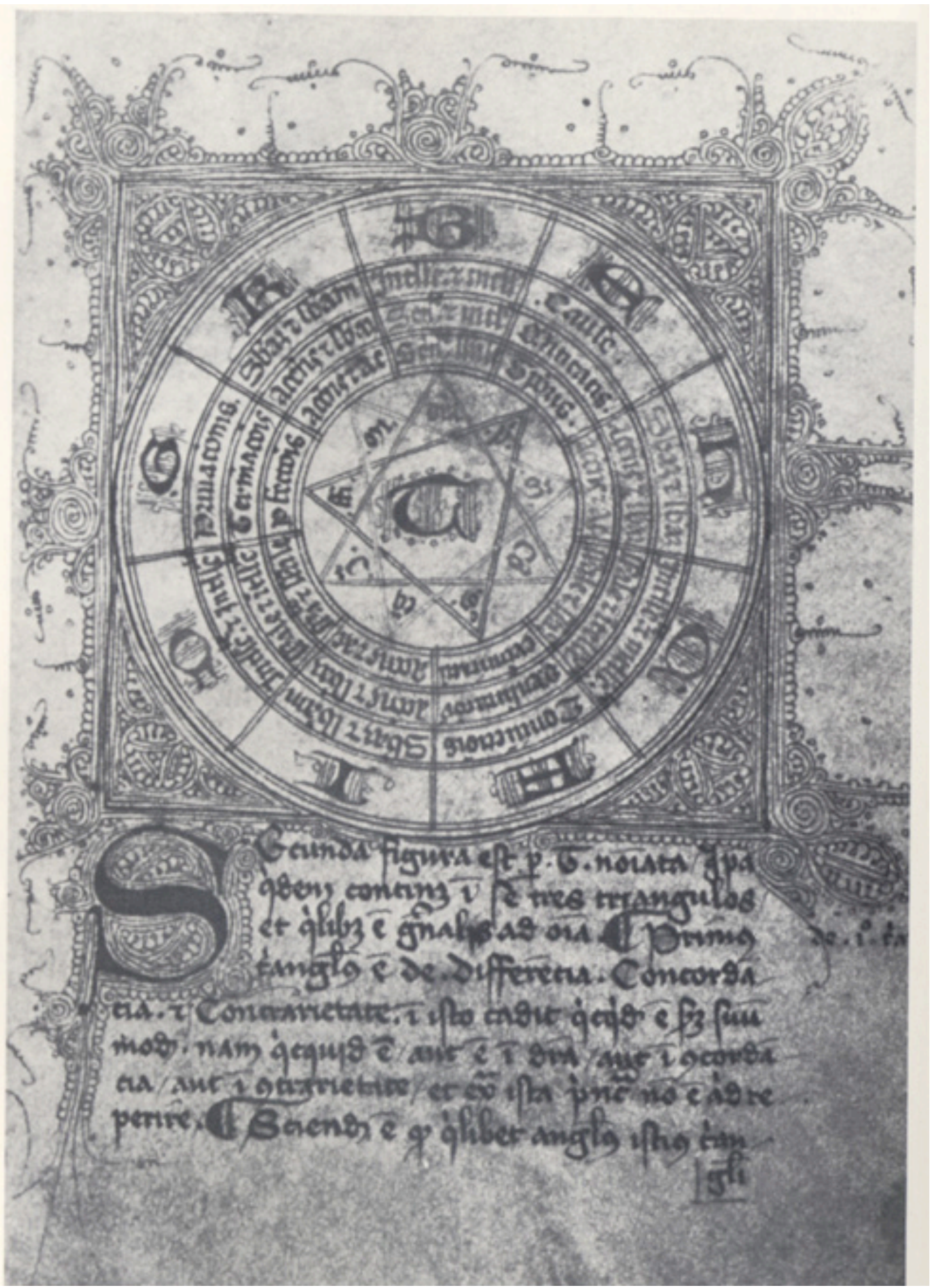

[ Figure 4: Llull's Figure T ] 
Figure $\mathrm{T}$ enables more active interpretative engagement on the part of a user when it is combined with the definitions expressed in Llull's Figure A to generate a chart, a structure technically known as a half matrix (Figure 5). Its appearance in this form alerts us that the chart is itself a further diagrammatic expression of some of Llull's principles of interpretation. Because Llull means his system to treat the combination "BC" as identical to "CB" it is possible for him to omit the symmetrical lower half of the matrix which would otherwise obtain, and since relational loops such as $\mathrm{BB}$ or $\mathrm{CC}$ are likewise not to be considered, Llull also omits the longest diagonal of the matrix (Bonner, “What Was Llull Up To?”). In this way, the very graphic organization of Llullian concepts participates in the expression of the enabling constraints under which the concepts are meant to function and through which they are enlivened.

Because the half matrix represents a combination of Llull's A and T circles, and all that they imply about the relations among their alphabetic ciphers, a simple enabling notation like "BC" becomes charged with meaning. B represents both goodness (from Figure A) and difference (from Figure T). C represents both greatness (from Figure A) and concordance (from Figure T). These embodied relations permit the generation for analysis of a phrase like "goodness has great difference and concordance." 


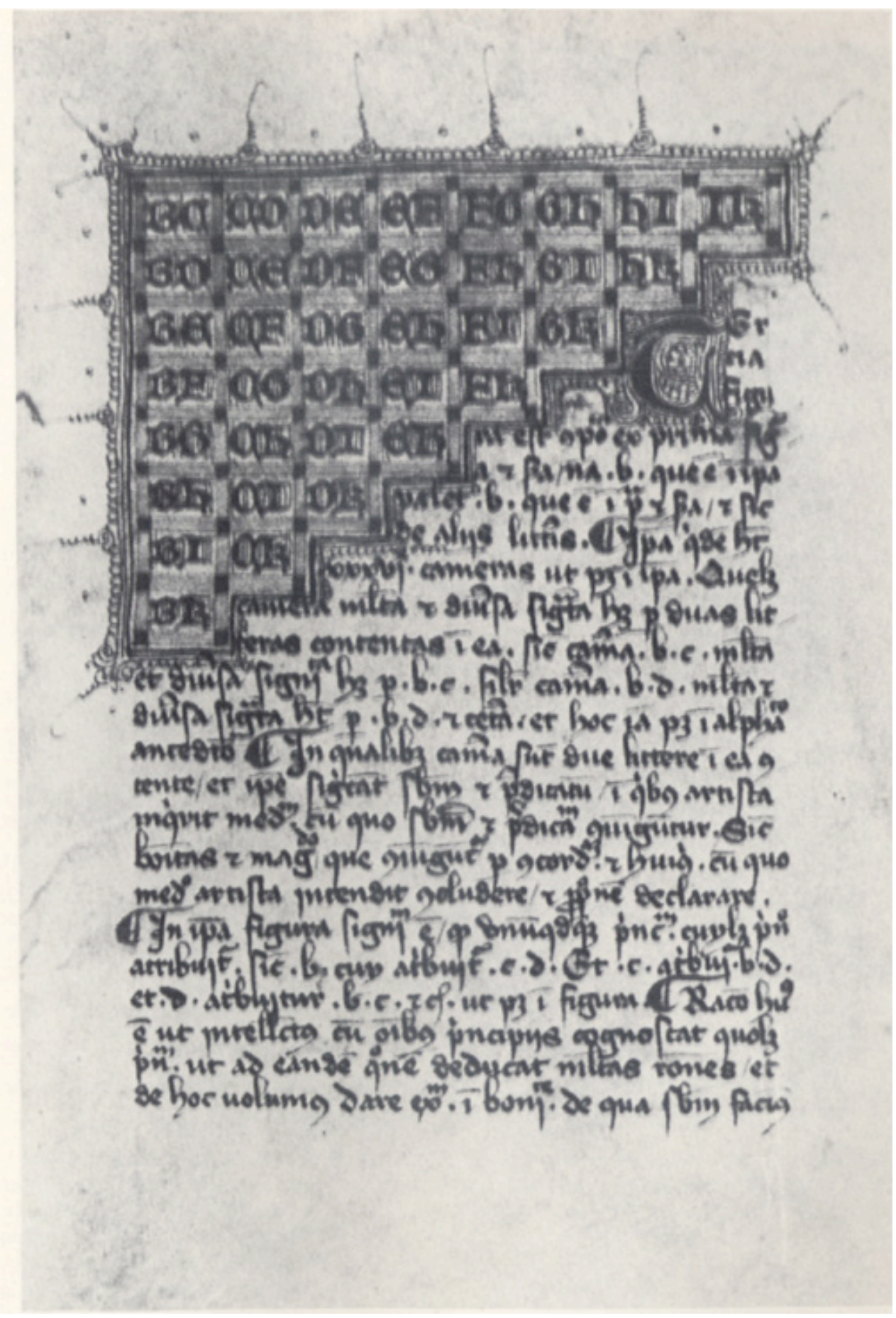

[ Figure 5: Llull's Third Figure, a half matrix ] 
An elevated pronouncement, indeed; but Llull takes steps to constrain — also through a generative process - output that could otherwise provoke an overly general discussion. When the concentric domain circles of Llull's Figure $\mathrm{T}$ are added to this equation, the user of the Ars Magna is prompted to analyze statements in terms of their applicability to a variety of specific sensual (or sense-perceptive) and intellectual relations. Llull offers an aid to such analysis, explaining these interpretive domains and their relation to the letter B (“difference") in the following terms:

There is a difference between sensual and sensual, as for instance between a stone and a tree. There is also a difference between the sensual and the intellectual, as for instance between body and soul. And there is furthermore a difference between intellectual and intellectual, as between soul and God. (Llull, Ars Brevis, qtd. in Bonner)

A statement like "goodness has great difference and concordance," then, is presented by Llull's circles not as an eternal truth, but rather in order that it be interpreted within a specified context - that of sensual and intellectual differences - and in all the embedded relations among those fundamental domains.

For all its complexity and utility in generating relational assertions, thus far the Great Art limits itself to binary structures, and to interpretations based on fundamentally invariable graphs and matrices. However, with the introduction of a novel fourth figure — in fact, a paper-based, codex-embedded device — Llull expands his system from 
binary into ternary relationships, and moves from abstract algorithm into the realm of mechanically-aided hermeneutic practice (Figure 6). He does this first by adding to the semantic weight of the primary alphabet a set of questions or — as he puts it interpretive "rules" and prompts: whether $(\mathrm{B})$, what $(\mathrm{C})$, of what $(\mathrm{D})$, why $(\mathrm{E})$, how much $(\mathrm{F})$, of what kind $(\mathrm{G})$, when $(\mathrm{H})$, where $(\mathrm{I})$, how and with what $(\mathrm{K})$. The prompts become part of a functioning ruleset for procedure and interpretation when they are inscribed, along with Llull's other encoded alphabets, on manipulable, inter-rotating wheels.

While versions of these wheels have been fashioned from a variety of media (including, most interestingly, the copper "covers" of a portable Italian Renaissance sundial masquerading as a book), they typically took the form of paper circles secured within incunables and manuscripts by small lengths of string (Dalton). The compartments or "camerae" of an outer circle would be inscribed on the page, while two inner circles were fastened on top of it in such a way as to permit them to rotate independently, mechanically generating interpretive problems based on ternary combinations of the inscribed alphabetic ciphers. 


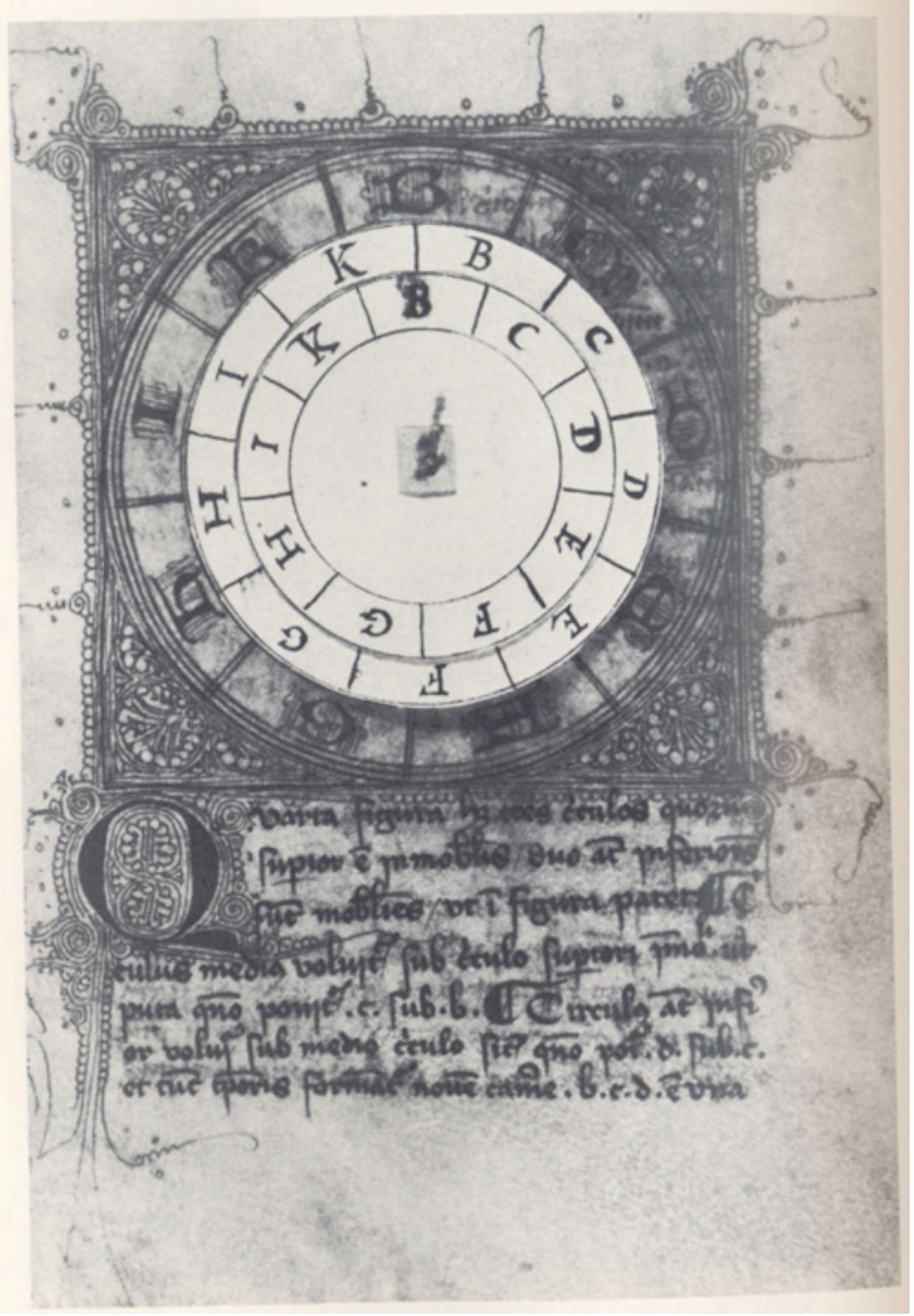

[ Figure 6: Llull's Fourth Figure, inter-rotating wheels ] 
In straightforward terms, the semantic combination of $\mathrm{B}$ and $\mathrm{C}$ then takes on six meaningful and relational concepts: goodness and greatness, difference and concordance, and the conditionals "whether" and "what.” Llull's wheels appear deceptively simple, but for the basic combination of $\mathrm{B}$ and $\mathrm{C}$ alone, they are capable of posing thirty-six issues to their human interpreters: twelve propositions (such as "goodness is great") and twenty-four questions or philosophical problems (like "what is great goodness?" and "whether goodness is great") multiplied down the succession of associations between, for example, goodness and difference, goodness and concordance, and so on. When three rather than two primary elements are combined with their associated questions or interpretive rules, as is enabled by the embedded, rotating wheels, even more complex problems can present themselves: for example, "whether goodness contains within itself difference and contrariety" (Bonner, "What Was Llull Up To?”).

Llull works out the results of his generative machine in tables similar to the half matrix used to express the simple relations of his first circular figure. In the Ars Brevis of 1308, a simplified version of his Great Art, the corresponding table has seven columns — but Llull's Ars Generalis Ultima presents the relations that emerge from expanded iterations of the rotating wheel concept in a table with no less than eighty-four long columns. Each alphabetic expression in these tables has been algorithmically, logically, and mechanically generated for human rhetorical and hermeneutic purposes. The 
cumulative effect is of an "extraordinary network of systems systematizing systems" (Bonner 574), and yet the Llullian apparatus exists in service of interpretive subjectivity.

Llull is thought to represent the "earliest attempt in the history of formal logic to employ geometrical diagrams for the purpose of discovering nonmathematical truths, and the first attempt to use a mechanical device — a kind of primitive logic machine to facilitate the operation of a logic system" (Gardner 1). Llull's wheels are often referred to as the "hardware" of this system, with the interpretive method he advocates for their use serving as "software," expressed, along with output from the devices, in "user manuals" like the Ars Generalis Ultima itself.

However, it is important to remember that most of the diagrammatic figures generated by Llull's wheels don't explore “truths” at all, but instead pose interesting queries and hypothetical situations for their users: for example, "when it might be prudent to become angry" or "when lust is the result of slothfulness." He also uses the wheels to help puzzle out such "typical medieval problems" as:

Could Adam and Eve have cohabited before they ate their first food? If a child is slain in the womb of a martyred mother, will it be saved by a baptism of blood? How do angels speak to each other? How do angels pass from one place to another in an instant of time? Can God make matter without form? Can He damn Peter and save Judas? Can a fallen angel repent? (Gardner 14) 
Llull's Book of the Ascent and Descent of the Intellect moves beyond the theological sphere to apply his method to eight categories of Nature to pose and suggest possible answers to scientific problems such as "where does the flame go when a candle is put out? Why does rue strengthen the eyes [while] onions weaken them? Where does the cold go when a stone is warmed?” (Gardner 15).

In the books accompanying his charts and diagrams, Llull sometimes offers full arguments and commentaries on such questions, sometimes outlines the combinatorial processes by which the questions could be addressed using his wheels, and sometimes simply demonstrates diagrammatically that such sophisticated questioning can be generated by means of the Ars Magna. At no point does Llull imply that his machine can produce "truth" independently from its human user, no matter how scientific his alphabetic abstractions appear. Instead, he himself tells us that the system employs "an alphabet in this art so that it can be used to make figures as well as to mix principles and rules for the purpose of investigating the truth" (qtd. in Zweig 22). That is, the mechanism enables interpretation through visualization by making the core elements it operates on and the rules by which it plays explicit. The flat generation of combinations is not the point of his Great Art: that's not hard to do. Instead, Llull provides his users not just with hardware, but also with a clearly-specified method for analyzing both process and output outside of the generative system — and more importantly, for refining that system iteratively, based on subjective human assessment of its mechanical 
output. Interpretation is the real activity of the Ars Magna, not the spinning of wheels.

Despite this fundamental hermeneutic interest, Llull's devices participate closely in two traditions exhibiting a vexed relationship with humanistic interpretation. Any "step by step" production of what Künzel terms interpretive "universes" is by nature an algorithmic production, and the mixing of principles and rules on which Llull's work depends is a nice elaboration of the notion of an ars combinatoria. An appreciation of both of these traditions and the methodologies that support them is critical to our understanding, not only of Llull and his interpretive devices, but also of new digital tools and environments being constructed by humanists to augment the methods and latitudes of modern scholarship.

\section{B. Performance and Interpretation}

\section{Algorithm}

Llull is often listed among the first philosophers "compelled to delineate clearly a general method" for deriving conclusions (Sales 2.3). Frances Yates goes so far as to assert that the "European search for method... began with Llull" (qtd in Zweig 23). We now commonly accept that "logical reasoning is, in a sense, computation" and that it “can be formalized and validated by controllable means" (Sales 2.1), but Llull's clear and materially embodied articulation of this concept has been seen as an advance in Western 
philosophy, constituting the first major formal extension of traditional mnemonics, a "now-forgotten integral part of medieval education: the complex set of elaborated techniques for reminding and structuring things in human memory in a printless age" (Yates). Perhaps more important, Llull's devices also implemented, for the first time in Western Europe, the newly-translated rule-based work of the Arabian mathematician Al-Khwarizmi, from whose name the word algorithm stems (Sales 3).

The relationship between algorithmic operation (as both a concrete and abstract methodology) and the design and use of interpretive toolsets like the Ars Magna is under-appreciated and perhaps easily misconstrued by liberal arts scholars outside of the tight community involved in building and making accessible the modern digital archive. Algorithms, when thought of as remote, inflexible mathematical structures underlying computer programming and the more deterministic branches of science and engineering, can seem irrelevant or even antithetical to the work of the humanities. By concentrating our efforts on the building of algorithmic "text analysis" tools, do we unthinkingly imply that the craft of scholarship can be mechanized? Are we tacitly putting algorithms forth as substitute for contemplation and insight? Or (a far more insidious assumption) are they, as the quiet servants delivering us the "content" of an archive, simply beneath our notice and contempt? In fact, algorithms — like various hermeneutic methods and historical schools of thought more dear to humanities scholars — can be understood as problem-solving and (with a slight methodological recasting I will suggest in a discussion of the "ludic algorithm") as open, participatory, 
explorative devices.

The algorithm is formally defined as a finite sequence of instructions, rules, or linear steps which, if followed, guarantees that its practitioner — whether a human or machine agent — will reach some particular, pre-defined goal or establish incontrovertibly that the goal is unreachable. The "guarantee" part of this description is important, as it differentiates algorithm from heuristic, or what is generally called a "rule of thumb." Like algorithms, heuristics can function iteratively to solve a problem and can be responsive to human input. Computer programs that modify themselves in response to their users, such as word processing spell-checkers, are sometimes — despite their algorithmic basis - termed heuristic. However, the heuristic process is fundamentally one of informal trial and error rather than constrained activity according to a set of predefined rules.

Almost any everyday problem can be solved heuristically or algorithmically. For example: I've lost my car keys. Ordinarily, a harried new mother faced with this situation will proceed by heuristics: I look in my purse. I look in my purse again. I brave the cluttered diaper bag. I check the front door, because I have developed a bad habit of leaving them dangling there. I go to the last place I remember holding them in my hand. I ask my husband if he's seen them. I wish the baby could talk. In formal, graph-based problem-solving, heuristics are sometimes used to guide the search for solutions, by identifying the most promising branches of a search tree for further exploration, or even by cutting out unpromising 
branches altogether. The weak point of the heuristic method becomes evident when its user needs to shift gears. I'm not finding my keys in the usual places. Should I retrace my steps next? Is it worth considering that I may have locked them inside the car? The basic "problem with heuristics" - in some cases a crippling problem, which could lead to a desired outcome's branch being wholly eliminated from the search tree - "is how to decide half-way what would be an appropriate next action, i.e. how to design heuristic rules that lead to good solutions instead of bad ones" (Lagus). Tellingly, we often attribute decisions in successful heuristic processes to intuition and those that result in undesirable outcomes to confusion and bad luck.

If the heuristic process fails or seems too unsystematic for comfort, a desperate searcher can always resort to a true algorithm:

For each room in the house; and

For each item in the room;

Pick up and examine the item.

If the item appears by objective criteria to be the missing object, terminate the search. If not, put down the item and continue this loop until all items have been tested.

Eventually, if this little program is executed perfectly, I will either find my keys or determine conclusively that they are not in the house. There's a kind of predestination or special providence about an algorithm, formally defined. That is to say, I know to expect one of these two outcomes before even undertaking the search process. And - 
as its strict definition requires — the algorithm is almost wholly generalizable. If I suspect I have left my keys at your house, I can run the process there. If the misplaced object is a watch, or a hat, the algorithm is equally applicable. (Of course, it isn't a very efficient algorithm, because it requires me, for example, to pick up and examine the house-cat — and to do so every time it saunters into a new room — but we can easily imagine more elegant versions of this basic method.)

Some common refinements to the concept of the algorithm are particularly relevant to interpretive or hermeneutic activity, which, by virtue of its humanistic realm of application, is generally predicated on ambiguity and flux. Algorithms are expected to be both perfectly precise and entirely implementable. An old bubblegum wrapper joke helps to make this point: How do you fit four elephants into a Volkswagen? The seemingly algorithmic answer is that you simply put two in the front seat and two in the back. While those steps are clearly unambiguous, they are however impossible to implement. In contrast is a commonplace algorithm for finishing one's dissertation:

Step 1: Write the next paragraph.

Step 2: Repeat Step 1 until dissertation is complete.

This procedure is clearly implementable - graduate students perform it all the time but it is far too ambiguous to be a textbook, or even a useful, algorithm. How exactly does one write a paragraph? What criteria indicate that the thing is "complete?" What 
is a "paragraph," anyway? How does the algorithm know that you're writing a "dissertation" and not a thesis, or a novel, or a comic book? (How do you know? That is to say, how determinable from the point of view of the algorithm's designer are the elements in this — in any — interpretive field?)

And so the algorithm, originally applied to mathematical operations and associated almost inextricably in the contemporary mind with computer science, emerges as a stepby-step, linear, precise, finite, and generalizable process that produces definitive, anticipated results by constraining the actions of the agent performing the process.

Almost as quickly as the application of algorithmic methodology to modern mechanical and computational apparatus became a fundamental aspect of design (with Charles Babbage’s 1837 “Analytical Engine”), algorithms themselves fell under fire as analytical or investigative devices. Babbage's colleague, Augusta Ada Byron King, Countess of Lovelace — the daughter of Lord Byron celebrated as the first computer programmer for her elaborations of the Jacquard loom-like cards on which the Engine operated famously critiqued the algorithm:

The Analytical Engine [and, by extension, the algorithmic method on which it is based] has no pretensions whatever to originate anything. It can do whatever we know how to order it to perform. It can follow analysis; but it has no power of anticipating any analytical relations or truths. Its province is to assist us in making available what we are already acquainted with. (Lovelace, "Note G") 
Lovelace's objection hinges on the reasonable idea that an algorithm can yield nothing more than its designer knew to ask it for in the first place. Algorithms are not fundamentally creative or revelatory. They merely perform pre-defined transformations and produce requested — and therefore anticipated or even presumed and therefore potentially flawed - results. We could see this quality, by way of example, in a purely mechanical performance of our car-key algorithm. The procedure's outcome (confirmation or disconfirmation of the presence of car keys) could be in no way unexpected; it is in fact built inextricably into the process. Algorithms are certainly applicable to problem solving, but Lovelace suggests that they only (perversely) solve problems whose answers are projected, which is to say pre-known.

The Lovelace Objection and its descendant Turing Machine critiques bear a striking resemblance to Martin Gardner's derisive description of Llull's Ars Magna as a means built toward inappropriate ends, and for the manipulation of intractable objects (Gardner 18). In such a case, any application of the algorithmic process to subjects for which, in McGann's formulation, "imagining what you don't know" is a desirable outcome seems misguided at best. At worst, the use of algorithmic process in an interpretive or humanistic context can be seen as self-delusion justified through pseudoscientific formalism. (Critiques of "frivolous" combinatorial and deformative text manipulations and dire warnings against AI optimism in our ability to apply computational methods to text analysis participate in this limited acceptance of the uses 
to which algorithms may be put.)

Algorithms admittedly define and constrain a field of activity, even as they enable certain pre-ordained interactions and solutions. Still, this is not to say that the results of algorithms - and even more, algorithmic methodology as subjective (most likely human) agents could actively and iteratively employ it — can't paradoxically expand our thinking rather than atomize it, or limit it to presumptive outcomes.

The precision a true algorithm requires of its elements and processes assumes a certain determinability and fixity of identity that is difficult if not impossible to maintain in interpretive fields. But to attempt, in data modelling or in performative criticism, an algorithmically-enforced specificity is to experience and exploit a productive brand of resistance in the materials of humanities scholarship. Real challenges and opportunities arise for expanding our understanding of such fields (including, at the most deceptively basic level, those graphic and textual artifacts we call books) in the rigorous and thoughtful application of algorithmic method to our analysis and manipulation of indeterminate objects and ideas.

Lovelace gets at these consequences of algorithmic method in a neglected passage immediately following her well-known “objection.” She explains that the Analytical Engine's facility in following rules and orders, producing expected results, and "making available what we are already acquainted with" is effected 
primarily and chiefly of course, through its executive faculties; but it is likely to exert an indirect and reciprocal influence on science itself in another manner. For, in so distributing and combining the truths and the formulae of analysis, that they may become most easily and rapidly amenable to the mechanical combinations of the engine, the relations and the nature of many subjects in that science are necessarily thrown into new lights, and more profoundly investigated. This is a decidedly indirect, and a somewhat speculative, consequence of such an invention. (Lovelace, "Note G")

Here Lovelace takes up, in the context of combinatorial mathematics, that product of algorithmic, diagrammatic, deformative, and mechanical method cited here under the broad rubric of "aesthetic provocation."

\section{Aesthetic Provocation}

Fostering of algorithmic and aesthetic provocation first came to my attention as an explicit, structuring goal for my work in designing digital tools for humanities interpretation during a series of instructive conversations with John Unsworth (then director of UVA's Institute for Advanced Technology in the Humanities), about the development of the Temporal Modelling PlaySpace, a specialized computational environment for promoting visual thinking (described in Chapter II below). The question at hand was the relation of on-screen information visualization techniques to the interpretation of humanities data, and more specifically the role of the computer as facilitator of — or participant in — the hermeneutic event. Unsworth's contention was 
that visualizations (defined in his formulation as any graphical manifestation of the abstract patterns embedded in data) are best produced algorithmically from information that has already been digitized, or entered into a computer system in an encoded form. ${ }^{3}$

This brand of visualization can be seen as a behind-the-scenes, culminant step in the process of digitization, made possible by the labor of content modelling and mark-up the step which, essentially, frees the human user of an electronic knowledge representation system from the necessary constraints of that system's construction. When previously marked data reach a state of complexity and size at which they lend themselves to algorithmic visualization techniques, the human interpreter can be presented with an "aesthetic provocation," a view of perhaps familiar information in a new format — a new picture calling for new exegesis.

We generally think of these provocations as taking visual form — word frequency in Alice in Wonderland becomes a set of bar graphs, author relations in Tottel's Miscellany are expressed as a color-coded network of linked nodes — but it is important to understand that any algorithmic transformation that opens a work or document to new interpretation is a variety of aesthetic provocation. The results of search engine queries fall into this category, as do those precursors of digital humanities, textual concordances and editorial apparatus appearing in and referring to the codex form. The only requirement here is that the new format be produced algorithmically from data marked or characterized by someone other than the end-user of the resource and that through the 
provocation, in Lovelace's terms, "the relations and the nature of many subjects... are necessarily thrown into new lights, and [can be] more profoundly investigated" ("Note G”).

This after-the-fact (after, that is, the data-marking or -modelling) application of aesthetic provocation is the principal manner in which information visualization enters the broader picture of humanities computing. This is in part because humanities computing itself, which came into proper being in the 1940 s with structured inquiries into the work of St. Thomas Aquinas by the Jesuit scholar Roberto Busa, has always orbited the double stars of corpus linguistics and database construction and mining. An intense emphasis on the encoding and analysis of (primarily textual) human artifacts, coupled with the pressures of re-constituting our shared archive and making it accessible in digital form, have contrived to make algorithmically-generated visualization - from the end-user's perspective - more a product to be received than a process in which to participate.

To some degree, this attitude toward algorithmically-generated visualization also reflects technical and institutional difficulties involved in processing, marking up, and producing meaningful images in a computational environment. In other words, texts divorced from their documentary instantiations and from the world of images - have been traditionally much easier to work with. And it is possible that the neglect of imaging by humanities computing scholars betrays a lack of training or interest in 
visual criticism in their native disciplines, or perhaps even a backlash against an undisciplined enthusiasm for pictures and animation evident on the World Wide Web. ${ }^{4}$ Regardless of the whys and wherefores, aesthetic provocation in the form of humanities computing information visualization is often, from the perspective of the end-user of a digital resource, inaccessibly "algorithmic" and after-the-fact.

That the algorithmic statements — in the form of text manipulations, graphic visualizations, Llullian ars combinatoria, et cetera — made possible by the systematizing work of database designers can be as novel and stimulating as any other acts of criticism is not in dispute. (The graphic representation of connections among Rossetti Archive documents presented in Chapter III below is a prime example of the ability of a visualization algorithm to draw attention to relations obscured in other data presentation formats.) We would be mistaken to assume — despite the fundamental validity of Lovelace's observation that algorithms "originate nothing" — that algorithmic output is not at the very least aesthetically provocative, and therefore of interest to humanities scholars. Aesthetic provocation, in its usual sense, is predicated on rhetorical revelation. ${ }^{5}$

Visionary computer scientist Alan Turing, in a noted critique of the Lovelace Objection, examines this revelatory propensity — the tendency of algorithmic mechanisms to provoke or surprise their users - and ultimately offers us a socialized, humanized view of algorithmic methodology. He begins the discussion with an attempt to reframe 
Lovelace:

A variant of Lady Lovelace's objection states that a machine can "never do anything really new." This may be parried for a moment with the saw, "There is nothing new under the sun." Who can be certain that "original work" that he has done was not simply the growth of the seed planted in him by teaching, or the effect of following well-known general principles? (Turing, Section 6)

These "well-known general principles" are perhaps commonly thought of by humanists as the informal, heuristic methods transferred to us over the course of a rich and varied education. (One would generally rather investigate this than that; when writing on this subject, one must avoid that quagmire; etc.) But what if Turing means us to understand our day-to-day practices in "following" these principles as little more than the playingout of socially-acquired algorithmic procedures, the output of which in a human context feels like originality, invention? In other words, might we not follow formal, specific (and wholly ingrained) rules even — or perhaps most of all — when we engage in our most creative and supposedly inventive work? What is it, precisely, that inspires us?

There is no question that algorithmic method as performed by humans or machines can produce unexpected (even if, as Lovelace points out, fundamentally predictable) and illuminative results. The religious traditions of gematria and Kabbalah, the conceptual art of Sol LeWitt, John Cage’s aleatory musical compositions, OuLiPian literary production, and the procedural experiments of Ron Silliman, Jackson Mac Low, and 
others (e.g. Lisa Samuels' poetic deformations) are primary examples of the inventive application of algorithmic method in the "analog" world. The inspirational power of constraining systems and algorithmic methodology is everywhere evident; it is the reason we have sestinas. In a practical, humanities computing context, computational algorithmic processes have been employed to perform revealing and sometimes startling graphical and statistical transformations under the rubric of text "analysis." And digital information artists like Benjamin Fry and W. Bradford Paley make exciting use of algorithms and strict systems of constraint in works that fruitfully blur the boundaries between creative and critical production. ${ }^{6}$

The contributions of cognitive science to the humanities over the past few decades have participated in what Colin Symes terms a "progressive demystification" of fundamental assumptions, long held in some quarters of the academy, about interpretive and artistic creativity. A Romantic vision of the artist unbound, as liberated in thought (a vision perhaps too easily countered with reference to the empowering constraints that drive even Romantic poetic practice), has given way among cognitive scientists to a growing "emphasis on the importance of a structured imagination" (Symes 87). ${ }^{7}$ According to this understanding, a top-down model of cognition which builds on Marvin Minsky's notion that mental framing devices both structure and filter our thought processes, creativity functions almost wholly through elaborate systems of constraint. The idea that, as Jon Elster posits, "artists tend to maximize their options through minimizing their choices," may strike some as counter-intuitive, but creative work in any number of 
disciplines bears this theory out, and it remains useful despite more contemporary critique (Elster, "Conventions;" see discussion in Chapter I. of MacEachren’s How Maps Work $)^{8}$

Perhaps equally peculiar is the suggestion that Minsky's framing system, which is structured hierarchically, could foster the subjective, non-hierarchical, out-of-the-box thinking we associate with interpretive and artistic production. According to this model of cognition, information filters progressively through top-level framing structures into lower-level “terminals.” Minsky’s primary interest is in the mechanisms of simple perception, but his concept of cognitive frames is equally applicable to more complex linguistic and creative processes. Uppermost frames in this case constitute a "range of primordial scripts" and "default grammars that control the structures of language" (Symes 88). There are, however, secondary constraining grammars. Margaret Boden terms these mental constraining systems, which structure critical and artistic thought and production within specific genres, forms, or disciplines, "computational spaces" (Boden). Non-hierarchical cognition is fostered therein through supporting structures "whose computational spaces or frameworks are derived from particular epistemological and aesthetic domains." These specialized spaces function both within and beyond the primary framing system that hosts them, generating, for instance, "forms of linguistic organization which transgress and even transcend those governing natural language" (Symes 88). 
Poetic composition provides a clear example of the use of meta-grammars both to organize and to provoke subjective response. This distinction between organization and provocation is an important one, because cognitive systems of constraint act simultaneously as matrices in which the fundamental units of language are placed, and as generative processes or algorithms. That is to say, a poet perceives the sophisticated metrical and rhythmic constraints of a sestina not simply as structures, but as a performative or procedural imperative. The linguistic patterns such constraints make impossible are as crucial to the composition of a poem as those they privilege and enforce. In this understanding of subjective response to algorithmic imperatives, poetry is shaped by what it cannot be, and poets by what their chosen forms will not let them do.

Such genre- and form-specific shaping may in fact become a physical or neurological condition of the performer. Cognitive scientist K. I. Foster has identified in the brain, with repeated linguistic use, a restructuring of the neural circuits or "connectionist pathways that excite mutually consistent arrays of language.” Interestingly, these pathways "at the same time inhibit those that are inconsistent with the exigencies of the constraint” (Symes 90). For the poet, the development of self-organizing mental systems results in a greater facility, over time, within his most familiar computational spaces and in the production of his chosen forms. And for this reason, writers exercise their faculties by engaging in rhetorical and metrical exercises and linguistic games, such as acrostics, bouts-rimés, or complex forms like hendecasyllabics. Gerard Manley Hopkins, who constructed poetic matrices of ever-increasing complexity, maintained (or 
perhaps sought to reassure himself) that "freedom is compatible with necessity" (Hopkins). In fact, scientific investigation of the processes underlying poiesis suggests that artistic freedom may only be embodied — both artifactually and physiologically — through the necessities of constraining and algorithmic systems. ${ }^{9}$

Experimental and synthetic work in analyzing literary expertise also tends to support a constraints-based reading of the poetic and interpretive process. Cognitive research by Marlene Scaramalia and Carl Bereiter indicates that the presence of strict constraining systems promotes greater linguistic fluency in writers, by lending "form and direction to the more localized decision-making" involved in word choice within a particular genre or format (Symes 89). In effect, as Jon Elster demonstrates, this concentrates creative energies by economizing on the number of aesthetic and subjective choices available to the artist at any one time ("Fullness," 164). Robert De Beaugrande explains the futility of any attempt at artistic composition unfettered by localized systems of constraint in terms of the "combinatoric explosion" that would occur should the range of choices become “unmanageable” (De Beaugrande).

The dual operation of computational spaces as structured matrices and generative algorithms functioning both within and beyond Minsky's top-down, framing filters becomes evident in our attempts at modelling and encoding the artworks these spaces engender. Poetic conventions generate linguistic artifacts that, despite the regularity their constraining patterns enforce, are essentially non-hierarchical. This fact is 
attested to by the infelicity of common text markup systems at capturing poetic (as opposed to informational) organization hierarchically. ${ }^{10}$ We should also note that constraint does not operate at the same, uniform scale throughout a creative or interpretive procedure, but rather shifts in specificity depending on choices made and exigencies encountered. And all these notions are complicated by a necessarily performative slant to any algorithmic or constraints-based methodology.

The acknowledged primacy of constraint in facilitating artistic production plays into a re-casting of (and some significant dissent over) the nature of intentional formalism by a small suite of associated 20th-century performative movements, each of which interrogated notions of constraint and algorithm. While, as in any artistic challenge, the lineaments of their debate were materially drawn, the disagreement between OuLiPo and its counterparts in Dada and Surrealism lay not in the objects these artists produced, but rather in their means or methods of production. How was enabling, ludic constraint best exploited, most fruitfully managed? The OuLiPian approach heightened formalism to dizzying levels and called on human creativity to scale appropriately. Dada, in a rejection of the lyrical subject, moved away from an inward-focused, traditionally "intentional" art and embraced mechanically-driven or random, generative composition. Surrealist art takes up some of the same methods and techniques as Dada and OuLiPo, but to different ends, attempting a return - through generative and aleatory forms - to a repressed, authentic, inner voice. 
Dada, seen as "the most elaborate expression of the anarchic aesthetic, and therein the most avid expression" of what OuLiPian critics like Colin Symes call a "denial of artistry," epitomized a push away from constrained creative production in its preference for found objects over crafted ones (Symes 90-91). Surrealist compositional practices verged even further from the synthetic imperatives of formal constraint in advocating randomness, chance - a host of "aleatoric procedures" meant to question traditional notions of authorship as controlled creation or decision-making within a structuring matrix. Artists began to appropriate external, even deliberately "inappropriate” systems of organization for their productions, and to devise working processes or algorithms that minimized their own subjective contributions to their artwork. This type of practice perpetuated itself in the experimental art of later generations, which has been interpreted as embodying a "negation of intentionality," a removal from the process — or at least a radical re-conception — of artistic subjectivity (Cramer and Gabriel). For instance, John Cage is known to have composed music by transcribing the positions of stars in the night sky onto a piece of tracing paper on which he had drawn a musical staff $(91) \cdot{ }^{11}$

A similar aesthetic, but with different implications and emerging from a radically different attitude toward process and organization, is evident in the creative efforts of OuLiPo, the Ouvroir de Littérature Potentielle. This group of mathematically-inclined writers positioned itself not only (as it is generally understood) against Romantic sensibility, but also in opposition to aleatoric Surrealism, even going so far as to self- 
identify as "l'anti-hasard" (Symes 91). Through the elaboration and use of ludic structuring mechanisms, OuLiPo called for a radical return of literature from randomness and so-called mindless mechanistic or algorithmic process to the conscious constraints and formalisms that had driven it for so long. The vehement opposition of OuLiPo to the differently-controlled energies of Dada and Surrealism brings a crucial distinction into focus.

Algorithmic process (that is, mechanistic procedural work, no matter how constrained the steps of that procedure may be) is not synonymous with the peculiar brand of ludic constraint evident in OuLiPian method. This is in part to say that method itself is not simply another word for formalism. The methods and algorithmic processes of the Surrealists (from the OuLiPian perspective adopted by critics like Symes, Cramer, and Gabriel) worked rhetorically to deny the role of constraint in performative aesthetic provocation, as a shaping force in human creativity and interpretive faculties. In fact, according to this critique, algorithmic method as employed by other procedural arts became in part a substitute for authorship. OuLiPo's heightened formalisms, in contrast, were positioned to bend all their rhetorical power toward the provocation of controlled creative response. The value in composition according to the strictures of OuLiPian constraint lay in the degree to which writers were spurred to find creative solutions to puzzling linguistic problems. If we accept the (admittedly arguable) idea that constraint works as an intensification of formalism in the service of aesthetic provocation - the creation of new (even imaginary) solutions - less participatory 
brands of algorithmic work can seem dependent upon mechanical procedures or methods running counter to human creativity, even resulting in predictable outcomes. The roll of the dice, despite Mallarme's objection ("Un coup de dés jamais n'abolira le hasard"), can always be made subject to a Lovelace Objection. For in the case of techniques employing a chance distribution of elements, algorithms that operate as closed systems, without opening themselves to iterative intervention on the part of a human performer, produce — as all proper algorithms do — anticipated, even predictable, results. Their final products may contain randomly generated or positioned elements, but such randomness occurs in predictable locales and to a paradoxically intended, intentional, even objectifying effect.

In the OuLiPian critique articulated by Colin Symes, "closed," aleatory Surrealist algorithms (as opposed to open, participatory constraining systems like elaborate poetic form) effectively short-circuit a subjective negotiation crucial to the development of understanding and of art. Their methods of use, which divorce creative (albeit implicitly structured) decision-making from the artistic process in favor of mechanical acquiescence to the fated found object — the Cagean dictates of the stars — result in artifactual and documentary manifestations of "pre-understanding." Strict procedural art (as characterized by OuLiPo and critics sympathetic to its aims) makes little room for those dialogic, iterative cycles of interpretation thought by hermeneutic philosophers to result in understanding and real poiesis. Instead, its algorithms (like all formally-defined algorithms) output precisely — and only — what they have been 
configured to produce. ${ }^{12}$

Cagean dissonance, a clang of tones changing with every performance, is in these terms a kind of illusion of difference. It is a product and method Max Bense, in a seminal discussion of what he termed "generative aesthetics," held to be fully "devoid of subjective interpretation" and for which all "specific elements of the aesthetic state... are pre-established" (Bense 57). If algorithms are designed to solve problems for which the answer is already fundamentally known, then simple, mindlessly obedient algorithmic work (in which, as in the typical "aesthetic provocation" paradigm of humanities computing, the algorithm is largely divorced from participatory intervention) is not fundamentally creative, although it might be productive or synthetic.

Participatory constraining systems like those favored by OuLiPo, in contrast, have been thought to provide access to the "computational spaces" in which linguistic and artistic creativity is fostered (Symes 88; Boden). By emphasizing and concentrating the artist's attention on those elements that — despite the structuring mechanisms of a given constraint — remain open to decision-making, such an interpretation of algorithmic method results in artifacts that are wholly deliberate, carefully crafted. Furthermore, these formalisms demand continual cogitation on the part of the artist, whose latitude for choice has been made clear and who is implicitly encouraged to weigh, balance, and reconfigure his choices. 
OuLiPian objections to Surrealist and Dadaistic method therefore came on two fronts. Fundamentally, Queneau and others saw the work of their counterparts as uncreative, mechanistic, and algorithmic in the sense that the processes involved resulted invariably in products that were predictable (despite the role of chance operation) and hence pre-understood. In this sense, the artistic and interpretive process was overdetermined. Compare, for instance, the Surrealist method of "coulage," or “involuntary sculpture," in which a molten substance like hot wax is poured into cool water, with Raymond Queneau's OuLiPian "Exercices de Style," where the same little incident is related in 99 different, deliberate voices or styles. Or see discussion of the role of authorial intention in the 1945 manifesto of Romanian surrealism, Gerashim Luca and Dolfi Trost's "Dialectique de Dialectique:"

We have returned to the problem of knowledge through images... by establishing a clear distinction between images produced by artistic means and images resulting from rigorously applied scientific procedures, such as the operation of chance or of automatism. We stand opposed to the tendency to reproduce, through symbols, certain valid theoretical contents by the use of pictorial techniques, and believe that the unknown that surrounds us can find a staggering materialization of the highest order in indecipherable images. In generally accepting until now pictorial reproductive means, surrealist painting will find that the way to its blossoming lies in the absurd use of aplastic, objective and entirely non-artistic procedures. (Luca and Trost, trans. Daniel C. Boyer) 
In complement to the critique of over-determinacy, OuLiPo also saw the Surrealist project as uncontrolled and self-deceptive. Surrealist assumptions that aleatoric procedures were liberating and that randomness and automatic, unconstrained compositional practices tapped into an uninhibited artistic subconscious were antithetical to an OuLiPian (almost Bensian) understanding of the workings of the mind. If the Surrealists were "wrong," their aleatory procedures became pointless, a form of "creative slavery" to "chance, automatism, and freedom" (Roubaud 87, Symes 94-5). And even if they were "right" about the nature of poiesis, they represented the elevation of what OuLiPo held to be the worst aspects of human thought and creativity: boundless, gratuitous subjectivity in the service of impulse.

Regardless, OuLiPo saw itself as the champion of a literature that was "conscious" and "voluntary," which is to say imaginative and deliberate. Self-imposed constraining or algorithmic structures, according to Colin Symes, "produced a system of composition based on a deterministic aesthetic, in which few creative decisions were left to chance, and in which the products of compositional accident and contingency were almost entirely eliminated” (Symes 95). Symes further observes that the effect of rigorous constraint is "the very obverse of an anarchic aesthetic" in that it results in diminished redundancy, "that factor in language that is crucial to eliminating potential ambiguity and opacity" (95). Not only was the OuLiPian process supposed to be more thoughtful, but its resultant products were meant to embody (oddly, given its ready embrace of 
controlled compositional subjectivity) a more disambiguated, informational approach to art than could Surrealist work.

OuLiPian constraint is sometimes dismissed as a "means to an end, a device for posing linguistic problems over which to triumph” (Symes 95). Jaques Bens concurs, suggesting an analogy between OuLiPo and "rats qui ont à construire le labyrinth dont il se proposent de sortir" (43). This view of the OuLiPian project only holds true if we privilege its ends over its means, the products of algorithmic work over the creative and constructive processes that generate them — if we assume that everything of value in this endeavor comes into play only at the moment the rats exit the maze. We may find it easier, of course, to hold such a view as critics of algorithmic art rather than as producers; after all, we often have nothing but the product of an artistic process to examine and critique. ${ }^{13}$ If, however, we take up Turing's performative (and Lovelace's speculative) interests in algorithm and look at our own constrained critical and interpretive methods in the same light, perhaps we are more likely to appreciate a different brand of "triumph" in such work — indeed, in algorithmic methodology on a broad scale, regardless of the complex alignments driving 20th-century art.

\section{The Ludic Algorithm}

In short, what may look inaccessibly, mechanistically algorithmic in (even) the OuLiPian project is better understood as evidence of a ludic algorithm, which we might define as a constrained, generative design situation, opening itself up — through 
performance by a subjective, interpretive agent — to participation, dialogue, inquiry, and play within its prescribed and proscriptive "computational spaces." This work may embed within itself a proposed method, but doesn't see its ultimate product as simply the output of a specified calculation or chance operation. In fact, the desired outcome of the ludic algorithm is sheer, performative and constructive enactment of the hermeneutic circle, the iterative "designerly" process we go through in triumphing over those interpretive or creative problems we pose ourselves. ${ }^{14}$ As Queneau observes: "l'appearance [of work derived from such dialogical algorithmic methods] est surréaliste... la méthode ne l'est pas" (125; Symes 95).

Turing touches on this brand of dialogue in his contemplation of the relationship between a machine (the very embodiment of algorithmic process) and its fallible, creative human interlocutor:

A better variant of the [Lovelace] objection says that a machine can never "take us by surprise." This statement is a more direct challenge and can be met directly. Machines take me by surprise with great frequency. This is largely because I do not do sufficient calculation to decide what to expect them to do, or rather because, although I do a calculation, I do it in a hurried, slipshod fashion, taking risks. Perhaps I say to myself, "I suppose the Voltage here ought to be the same as there: anyway let's assume it is." Naturally I am often wrong, and the result is a surprise for me for by the time the experiment is done these assumptions have been forgotten. These admissions lay me open to lectures on 
the subject of my vicious ways, but do not throw any doubt on my credibility when I testify to the surprises I experience.

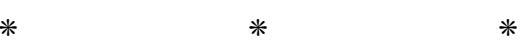

The view that machines cannot give rise to surprises is due, I believe, to a fallacy to which philosophers and mathematicians are particularly subject. This is the assumption that as soon as a fact is presented to a mind all consequences of that fact spring into the mind simultaneously with it. It is a very useful assumption under many circumstances, but one too easily forgets that it is false. A natural consequence of doing so is that one then assumes that there is no virtue in the mere working out of consequences from data and general principles. (Turing, Section 6)

If its performative and cooperative components are not appreciated, Turing's notion of algorithmic surprise lends itself to justifying a grossly limited vision of the interpretive activity possible in digital environments, an idea of algorithm that restricts its application to after-the-fact "aesthetic provocation." In fact, the real "surprise" involved here is less a matter of the algorithm working to its inevitable result on a set of data (as in a conventional information visualization) than of what that action, under observation, reveals about human thought processes. Turing is not a passive recipient of algorithmic output, but rather a predictive, constructive participant in its fashioning and reception. He makes assumptions, holds expectations, and awaits algorithmic response as just another part of a feedback loop. He is, in this, a reader of algorithms and their output, 
just as we all readers of the machine of the book. Still, despite the cumulative (socializing and humanizing) effect of Turing's assessment, as Stephen Ramsay reminds us, "to speak of an algorithm is usually to speak of unerring processes and irrefragable answers" - not of the participatory and iterative work of humanities interpretation (Ramsay, “Text and Algorithm”).

Turing's vision of the imperfect, risk-taking, intuitive human in conversation with a precise, calculating, fundamentally surprising machine partner is now familiar to us not only from science fiction and technological speculation but from our daily lives. We experience this brand of surprise perhaps most often as frustration in our interaction with algorithmic mechanisms like computers, ATMs, voice-response systems, handheld devices, and the DMV — interaction that can make us feel more like passive victims than active participants. We must realize, however, that Turing is documenting a fresh brand of dialectic, and by casting their facility in the "mere working out of consequences from data and general principles" as an anthropomorphized virtue machines can model for and perhaps teach us, he effectively rehabilitates computer-mediated algorithmic method as a creative and critical mode of performance. Recognition of the value of "working out... consequences" is as tangible a benefit, and perhaps as great a "surprise," as the mechanically-generated results of any imaginable algorithm. Performance (including human performance of algorithmic action) is to be valued here over passive reception. Turing's surprises are provocations to further action, not those "answers to enigmas in which we can rest” decried by James. That is, we are sure from his 
description and subsequent proposals (indeed from the whole character of his project) that Turing means to take the dialogue further.

My own desire for an enhancement of the typical aesthetic provocation paradigm (an enhancement we were later to embody in the Temporal Modelling PlaySpace environment) hinges — like Turing's observation and like OuLiPian practice generally - on the methodological uses of algorithmic constraint and calls for a new, more ludic and performative application of the notion of "aesthetic provocation." The problem with a visualization (or any other last-step provocation to interpretation) generated algorithmically from previously encoded data is that pre-encoded data is pre-interpreted data. And programmed algorithms that are flatly, "automagically" applied to a dataset, not opening themselves up to examination and modification by a user, filter the object of interpretation even further. The user of such a system is not properly figured as a user at all, but rather becomes an audience to statements being made by the designers of the system's content model and visualization or other representational algorithms.

While these statements can constitute — in all fairness — remarkable critical moves on their own part, the culminant effect of an unbalanced use of this approach is to reinforce a mistaken notion that digitization (and the concomitant application of algorithmic process of any sort) is a pre-critical activity, the work of a service industry providing socalled "content" to scholars. As an interpreter of algorithmic statements, a scholar (the end-user) is of course enfranchised to respond critically or creatively in conventional 
ways: by writing, speaking, teaching, or even by answering visualizations in kind, responding with new images. All of these responses, however, must take place outside of the system that provokes them, and to date (despite early encouragement by groups such as the MLA's committee on scholarly electronic editions), few digital projects have created real opportunities for critical engagement on the part of users. ${ }^{15}$ Sadly, the scholar's interpretive act plays a distant second to the primary interpretation or algorithmic performance encoded by the creators of most allegedly "interactive" digital environments.

A more fruitful interest in algorithms and algorithmic processes — as first embodied in Llull's combinatoric wheels and later articulated in OuLiPian schemata — lies in their design and our subjective experience in using them, rather than in their (oddly, at once) objective and Delphic output. A suggestion that we computing humanists move beyond the conventional application of "aesthetic provocation" is by no means a denigration of the measured use of traditional information visualization — of the algorithmic "product." The work outlined in this document, however, is much more invested in digitally or mechanically-assisted algorithmic methodology as an interpretive strategy. How are such provocative statements as those made by Fry's Valence or Paley's TextArc produced? Can we insinuate ourselves (our subjective responses, interpretations, participatory acts) more deeply into their production? We may find that the greater understanding of algorithmic process we gain in dialogue and cocreation with our Turing machines leads to a deeper appreciation of the self-replicant, 
recombinant documentary world in which humanities scholars live and move and have their being. For even the most pedestrian algorithmic construct opens itself up as an interpretive field in productive ways. Our simple car-key algorithm, for example, could easily, in performance, become a synthetic, interpretive, and creative ludic exercise - a game. ${ }^{16}$

Even at its most basic level — setting aside the intimate manipulations of a designer or programmer - algorithmic performance by subjective agents is revelatory. Imagine actually going through the prescribed physical process of picking up every item in your house, individually, and examining it for car-key-ness or not-car-key-ness. You might well find your keys by the end of the algorithm — but, by that time, the "success" of the operation would certainly seem beside the point. Undertaking this structured, constraints-based activity as a thinking human being, either practically or imaginatively, means more than performing it mechanically with one end in sight (confirmation or disconfirmation of the presence of car keys). Instead, you would be prompted continually to interpret and reinterpret your environment, your goal, your scope of activity, and your very actions, simply because a constraining system was compelling you to think algorithmically. You would, in performance, act on and reinterpret the objects of your ruleset and the ruleset alike.

Repositioning closed, mechanical or computational operations as participatory ludic algorithms requires acknowledgement of a primary definition, derived from the studies 
of game theorist Martin Shubik, a figure sadly neglected by academic game critics or ludologists. He concludes a powerful survey of "the scope of gaming" with the simple statement that "all games call for an explicit consideration of the role of the rules" (Shubik 34). Shubik means us to understand this "consideration" not only as adherence by players to a set of constraints, but also as appreciation of the impact of rules on the whole scope of play. The ruleset or constraining algorithm in any ludic system becomes another player in the process and, as expert gamers often testify, can seem to open itself to interpretation and subjective response - in some cases, to real, iterative (which in this case is to say, turn-based) modification. ${ }^{17}$ In our "consideration of the role of the rules" we must follow C. S. Peirce, and understand algorithmic rulesets "in the sense in which we speak of the 'rules' of algebra; that is, as a permission under strictly defined conditions" (4.361). The permission granted here is not only to perform but also to reimagine and reconfigure.

Seen in this light, the concept of deformance, made explicit as a critical practice by McGann and Samuels, is not so alien to traditions of literary interpretation as its critics may contend. Perhaps it is simply too easy for practitioners, in their own translations of deformative activities into verbal and illustrative explications, to emphasize the products of deformance (with their appearance surréaliste) over the interactive play involved in forming them. In fact, any act of translation or representation, especially algorithmic ones (or ones in which the algorithmic basis of operation is laid bare) can be seen as an exercise in deformative criticism (McGann 109-116). It is no accident that 
deformance stems from the twin performative traditions of art and editing. However, digital deformance machines and algorithmic generators of "aesthetic provocation" may seem far more interesting and open-ended to their developers during production than to their users and to their academic audiences later on. What many of them lack in use is present indeed during the development process — the kind of iterative adjustment based on human perception and intuition that open, dialogic instruments like Llull's Ars Magna enable.

Consider a notable example: Jerome McGann’s experiments in using Photoshop imaging software filters to produce “deformed” views of Dante Gabriel Rossetti's The Blessed Damozel. The manipulations became "interesting" at some point when the creative process fused with the analytical and mechanical process and McGann's adjustments created a "satisfying" image. Simple, automated application of a single embossing filter certainly revealed structural features of the painting that were less obvious (although still in some sense resolutely present) in its unaltered state — but noone who views McGann's deformations finds that instructive machine-generated deformance of the Damozel more compelling in what it seems to say about Rossetti's art than the intuitive and iterative (machine-aided) and practically unreplicable rainbowsmeared Damozel that follows (Figure 7; McGann, "Radiant Textuality"). The embedded subjective origin of interpretation evident in the second image - our instinctive notion that it encodes and embodies some individual perspective on the work rather than being the simple display of results from an "unerring" and "irrefragable" 
process - lends this version of the painting its power. As McGann puts it, the original "comes back to us through [the deformation] as if from an unimaginable world or point of view" (86).

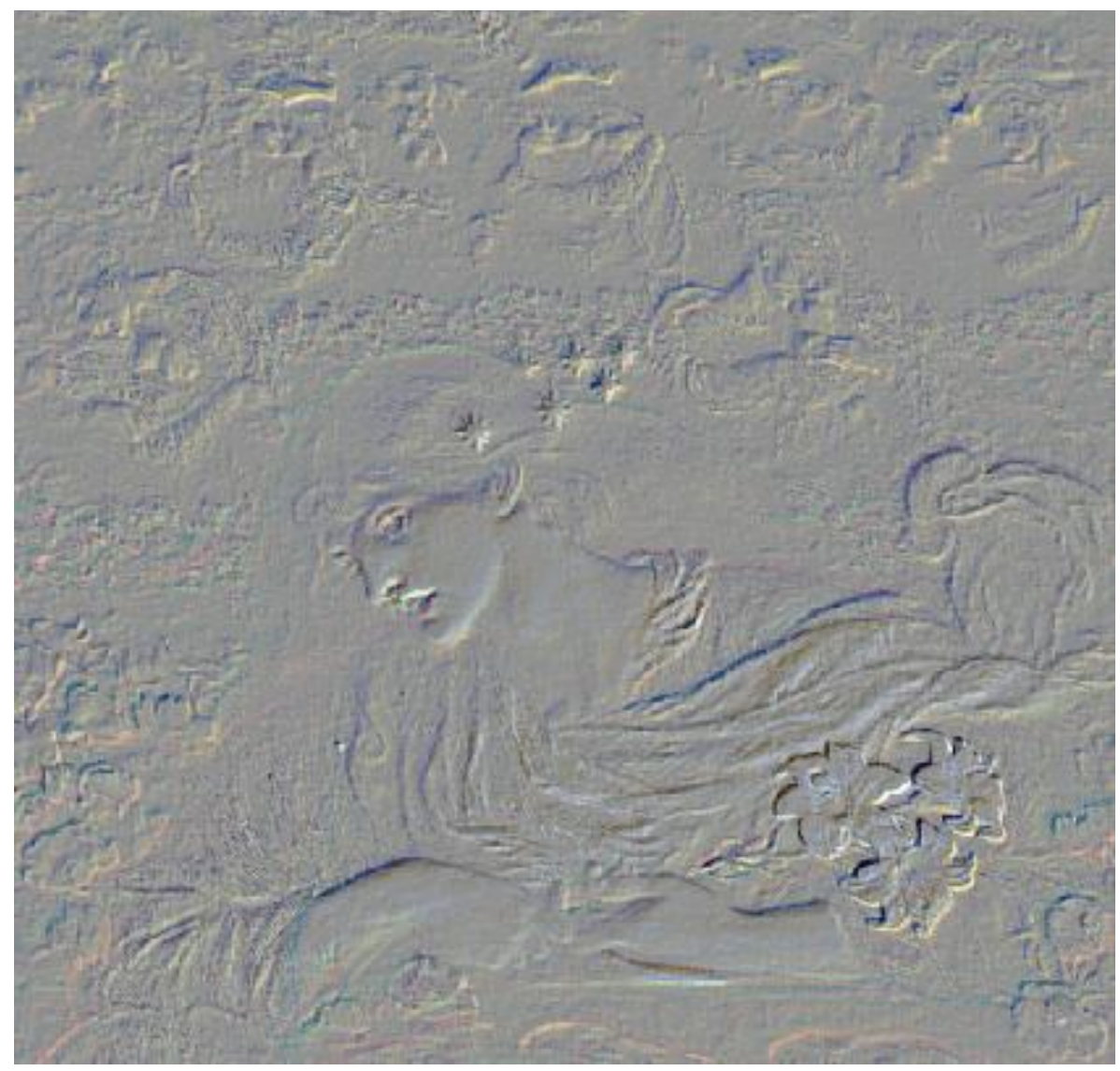

[ Figure 7a: Simple Algorithmic Deformation of Rossetti's “Blessed Damozel”] 


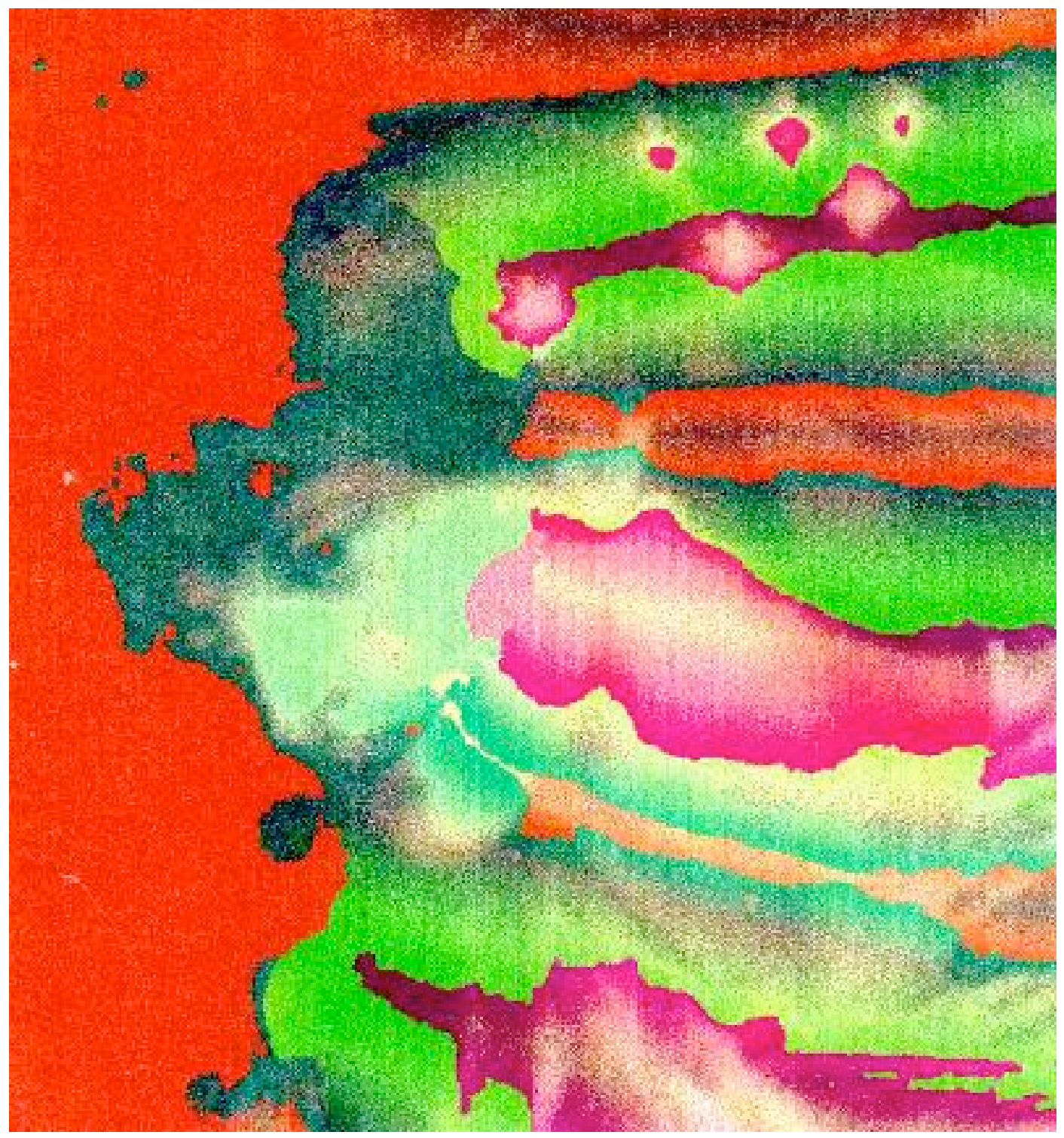

[ Figure 7b: Intuitive, Computer-Assisted Deformation

of Rossetti’s “Blessed Damozel” ] 


\section{Llull in Application}

\section{A Crisis in Communication}

Algorithmic and ludic operations, however fundamental to artistic and scholarly activity, remain exotic concepts to most humanists. Ramon Llull, our benchmark designer of the participatory, ludic algorithm, is more generally understood and discussed among academics in the historical context of ars combinatoria, a practice described by Janet $Z$ weig and others as rooted in mysticism and divination and leading up to the occasionally alienating aleatory experimentation of modern conceptual artists, composers, and mathematically-inspired writers (Zweig). Ars combinatoria have been called "the software of the baroque," with an output as rich as Bach's fugues, at once mechanical and occult (Bexte).

Anthony Bonner, in tracing the evolution of Llull's design from early forms more dependent on prose description, reference tables, and static figures, draws attention to the shift toward an ars combinatoria brought about with the introduction of the interrotating wheel:

Initially it appears as a device to compensate for the loss of basic principles that formerly constituted the building blocks of the Art; but soon one sees that it is in fact the replacement of a vast sprawling structure, whose parts are loosely and often only implictly (or analogically) interrelated, by a far more compact structure, whose parts are tightly and much more explictly and mechanically interrelated. (Bonner 573) 
Not only does the device, first embodied as the Fourth Figure of the Ars Brevis, serve that work's aim of making plain the complexities of Llull's Ars Magna, it also demonstrates that the essence of a "vast sprawling" and analogical structure can be usefully represented as a combinatoric art — so long as any shift toward concretization and precision is matched by flexibility in an open, interpretive ruleset.

Unfortunately, an ars combinatoria association for Ramon Llull's Great Art implies for some a focus that is either mystical (almost alchemical in scope) or tied up in an un- or pre-critical new artistic value placed on "pure process and play" (Zweig 23). What relevance can such flights of fancy have to serious scholarly issues of interpretation and analysis? We can begin to answer this question (an answer which can in fact only be embodied in the design and production of new tools) by contextualizing Llull's own design. His algorithmic and combinatorial device emerged not from mysticism or playful experimentation, but rather from a crisis in communication and interpretation. The Ars Magna was meant serve as an aid to hermeneutic thought and cross-cultural understanding in light of seemingly insurmountable (and unassailably rigorous) problems of textual criticism and rescension. That they seem playful in use is a mere fringe benefit of the serious interpretive burden Llull meant his spinning wheels to bear.

Llull was born on Majorca, only a few years after the King of Aragon and Catalonia had retaken the island from its Islamic conquerors. In Llull's time, Majorca was a melting pot: at least one third of the population was Muslim, there was a strong and influential 
Jewish minority in the economic and political center, and the rest of the island's inhabitants were Catholic. Künzel calls the Mediterranean of Llull's day "a kind of interface for three expanded cultural streams" (Künzel). Llull recognized many elementary commonalities among the three combative monotheistic religions represented on Majorca, but despite the sharing of basic concepts and notions of divinity, cultural tensions grew and Llull became deeply committed to the cause of resolution. We find it therefore "necessary to regard his invention as embedded within a special situation, i.e. embedded in a deep crisis of communication” (Künzel). Admittedly, Llull saw himself as a Christian missionary and his tools as enabling devices for the conversion of the infidels - not by the sword, as the failed Crusades had attempted, but by logical reasoning facilitated through the innovative combination of familiar, shared ideas.

Earlier attempts at peacefully convincing unbelievers, Llull recognized, had failed because of problems inherent to bibliographical analysis and textual criticism: theologians from the various camps had "based their arguments on sacred texts" (trying to point out errors in the Koran, the Talmud, or the Bible) — a practice which "invariably became bogged down in arguments as to which texts were acceptable to whom and how to interpret them” (Bonner, "What Was Llull Up To?”). A passage from Llull's Book of the Gentile and the Three Wise Men - written circa 1275 as a popular companion to the Ars Magna, in which the complex operands of that method are softened through presentation as the flowers and leaves of a tree - demonstrates the 
author's consciousness of the text-critical nature of religious problems of his day:

"I am quite satisfied," said the Gentile to the Jew, with what you have told me; but please tell me the truth: do Christians and Saracens both believe in the Law you mention?”

The Jew replied: "Christians and Saracens indeed believe that God gave the Law to Moses, and each believes that our Law is true. But because they believe other things that are contrary to our Law, therefore, and insofar as they believe these things contrary to our Law, they disbelieve our Law. Moreover, we and the Christians agree on the text of the Law, but we disagree in interpretation and commentaries, where we reach contrary conclusions. Therefore we cannot reach agreement based on authorities and must seek necessary arguments by which we can agree. The Saracens agree with us partly over the text, and partly not; this is why they say we have changed the text of the Law, and we say they use a text contrary to ours.” (Book II.3.6; trans. Bonner)

The innovation of the Ars Magna was to abstract philosophical concepts in play from their textual condition, by identifying notions common to the documentary sources of all three major religions and offering a combinatorial method for fusing them together and analyzing their relations. Llull's hope was that Christian arguments inspired by the Ars Magna would be satisfactory to Muslims and Jews, stemming as they did from logical combinations of their own basic beliefs. There is, however, no quality or assumption inherent in the Llullian method to enforce a certain interpretive slant. It's 
just as easy to use Llull's wheels to formulate arguments that favor Judaism or Islam.

All the interpretive impetus is placed on the artista, the human user of the Ars Magna. ${ }^{18}$

A similar and perhaps no less ideologically-charged crisis in communication plagues the academic community today. A crucial difference, however, lies in the fact that many of the principal interlocutors don't seem to realize (or at least acknowledge) that they are involved in a conversation at all. The subject is the role that digital media, inherently algorithmic and combinatoric, will play in the impending transformation of the scholarly archive. What are the appropriate structures for housing and describing our cultural texts and artifacts? With what tools might we undertake analysis and criticism in the years to come? The generative game described at the close of this chapter forms one imaginary solution to such problems.

\section{Dynamic Diagrams}

Llull's method (that first step in the Western "search for method") was not only notable for being clearly delineated; it was also self-testing, in the sense that the execution of iterative combinatorial motions was only carried out until contradictions or obvious untruths emerged. These untruths, naturally, would not appear as a parsing error or blue-screen breakdown in any material system at hand (the wheels, the diagrams), but rather in the conceptual system taking shape over the course of interaction with the Ars Magna in the mind of its user. At that point, the wheels themselves (and therefore all the marked primitives and practiced algorithms in play) could be examined and 
reconfigured. In this way, Llull's Art was both a generative and autopoietic mechanism, through which new posited truths and refined combinatorial and analytic methods could emerge.

Emergence, rather than derivation, is in fact the hallmark of Llullian method. The diagrams generated by Llull's wheels operate on principles of equivalency, not causeand-effect, generating statements "per aequiparantium, or by means of equivalent relations," in which ideas are not chained causally (the primary method for deriving logical and predictive relations), but are instead traced "back to a common origin" (Gardner 13). In the same way, Llull's idea of an ars combinatoria is not flatly combinatoric, but also fundamentally relational in structure and scope, in the manner of modern semantic tableaux (Sales 2.4). Even better, for Llull's uses, is the fact than this inherent value placed on human associations and the interpretive interplay of concepts ensures that Laputian "wisdom” or random nonsense can be rejected. We must, in looking at Llull's diagrams, appreciate his attitude toward their primary elements, the “constants" represented by an alphabetic notation. ${ }^{19}$ In Llull's estimation, nothing in the world was inactive. Nothing simply is; rather, everything does whatever its nature dictates it do. So Llull's emergent definitions (for example, the wheels may be spun to generate the simple statement "Goodness is Great"), which "to some commentators have seemed simply tautological, in fact imply a dynamic reality articulated in a large web of interactions” (Bonner, “What Was Llull Up To?”). Llull's definitions for alphabetic ciphers are "purely functional," after the style of "modern mathematicians, who do not 
say what a thing is, but only what it does" (Bonner 573). This dynamism provokes computer scientists like Ton Sales to argue that Llull invented the graph.

It is clear that "concept-structuring or taxonomic" graphical designs predate Llull (Bonner, “What Was Llull Up To?”). Tree-structures, for example, have been used to present genealogical or classificatory relations since the late Roman period. Llull himself, in a treatise on the Principles of Medicine, employs a (characteristically complex and dynamic) tree structure to express the relations among the bodily humors, the schools of medicine, and various aspects of health (Figure 8). ${ }^{20}$

Llull's typical graph was not built on the static, taxonomic model, but "conceived rather as a present-day's 'semantic network' and intended to be 'followed,' i.e. dynamically executed as though it were truly a fact-finding 'program' or a decision tree” as used in AI decision procedures (Sales 2.7). Such an image was therefore not a chart or illustration, but instead an "actual net of links that allowed the user to explore in a combinatorial fashion the relations that existed among the currently manipulated concepts” (Sales 2.9). In this way, Llull's designs resembled or prefigured modern conceptual graphs and semantic networks, as they "presupposed a dynamic interpretation" in which to know the concepts at hand meant to follow and explore their consequences and associations, to participate actively in the manufacture and representation of knowledge (Figure 9; Sales 2.9). ${ }^{21}$ 


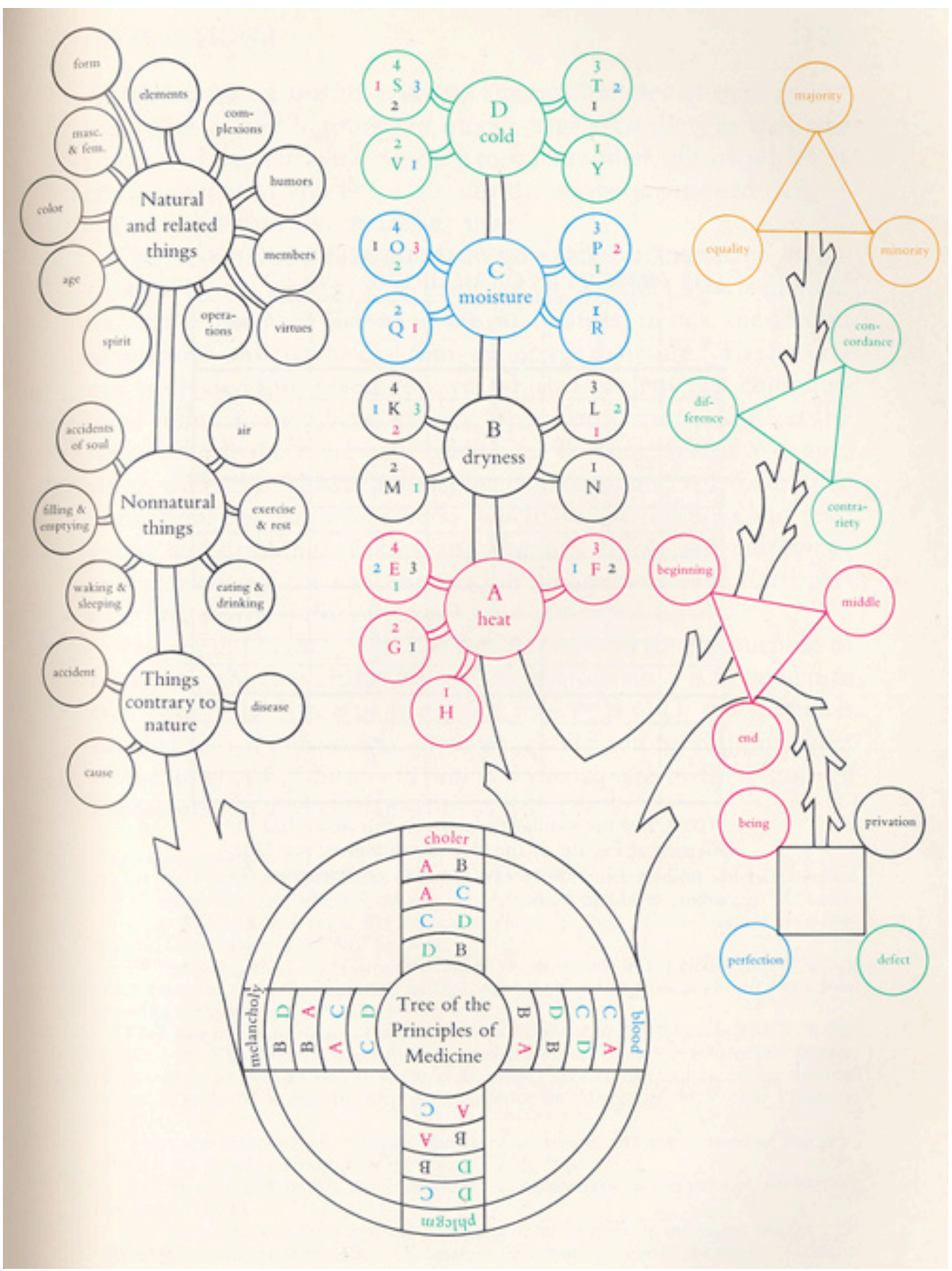

[ Figure 8: "Llullian Tree Structure"] 


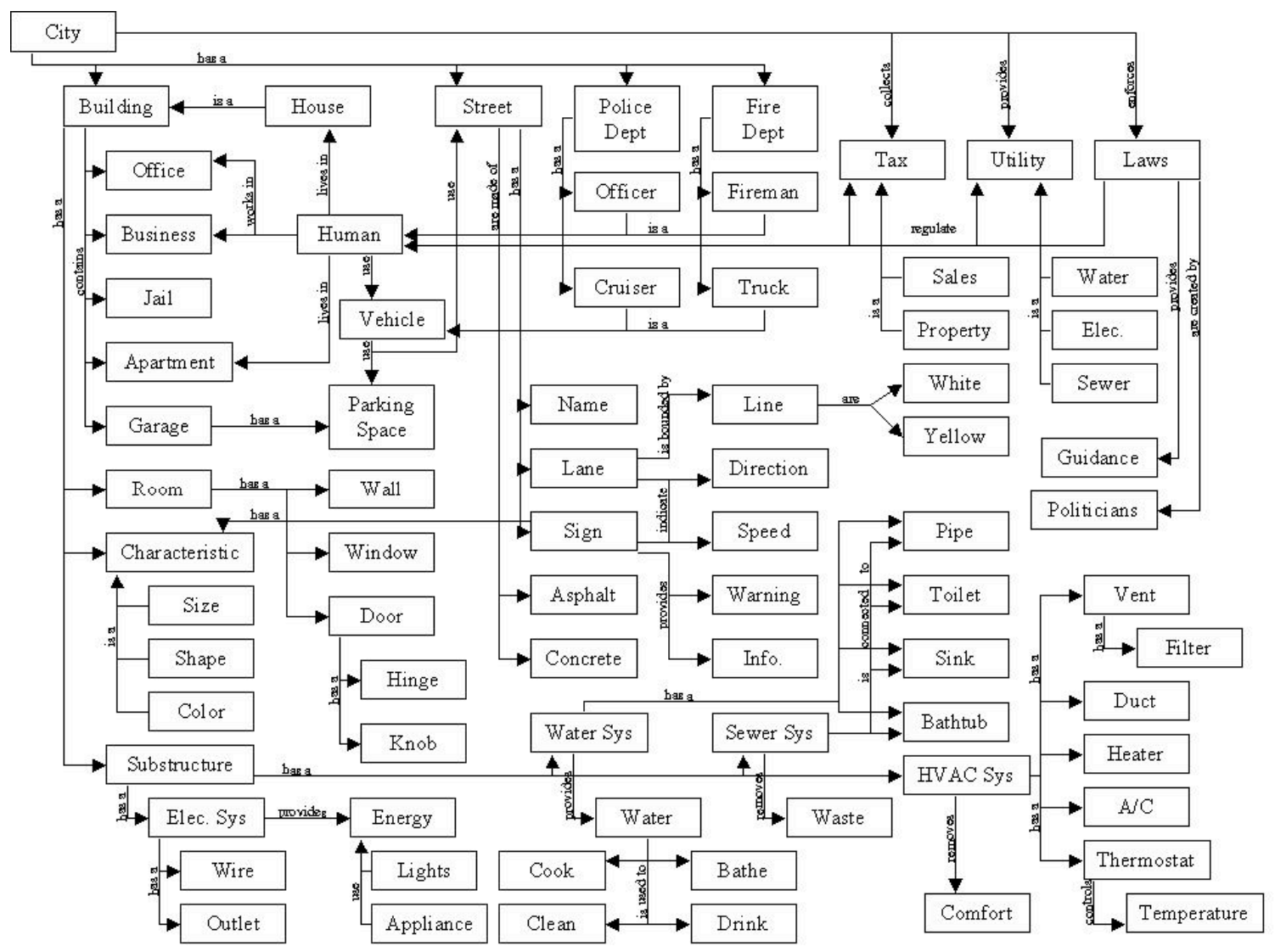

[ Figure 9: "Modern Semantic Network"]

\section{A Qualitative Difference}

Perhaps the finest quality of Llull's now-neglected system is that it assumes activity at all its levels. It works mechanically, graphically, and iteratively, and it asks its users to go even further in making their interpretive response to its combinatoric output in a purely interactive context. Here, we are not asked to feed data into a number cruncher or closed text-analysis system (the algorithms of which were perhaps fashioned by 
others, necessarily for other purposes and materials than our own) and wait passively for a report. We are instead meant to create, mark, and manipulate a wheel, to record its statements diagrammatically, and to follow and explore those resultant diagrams as a means of formulating, testing, and refining both ideas and rules, or algorithmic and combinatorial systems of interpretive constraint. No satanic mill, Llull's open-ended mechanical model instead follows Blake’s imperative: "I must create my own System, or be enslaved by another Man’s.” For no matter how benign and even profitable the typical enslavement to after-the-fact "aesthetic provocation" in humanities computing tools may be, algorithmic instruments that don't work on Llull's principle can only deliver us "answers" that are either pre-known or inaccessibly random - that is, either derivative from algorithms and content models that express deep-seated, framing preconceptions about our field of study (as in typical, last-stage "aesthetic provocation"), or derivative from deformative automations (like the embossed Damozel of Figure 7a) that too often do not open themselves adequately to the participation of a subjective agent during their operation. ${ }^{22}$

Janet Zweig, in her overview of ancient and modern ars combinatoria, asks a fundamental question, relevant to appreciating Ramon Llull and his Great Art in the context of digital humanities and computer-assisted hermeneutics: "What is the qualitative difference between permutational systems that are intentionally driven and those systems that are manipulated with chance operations?” (Zweig 20). It is important to understand - as Llull's critics and the slow forces that have driven him into obscurity 
did not — that the Ars Magna is not highfalutin, theological Twister: a governing, user-manipulating system of chance operations and random (or worse — insidiously circular) output.

Zweig's question about the qualitative difference between aleatory and intentionallydriven mechanisms implies its own answer: the differences are qualitative, embedded in and emergent from our familiar world of humanistic interpretation. We're not meant merely to get output from Llull's wheels. They are designed to generate insight into their own semi-mechanical processes and into our rhetorical and hermeneutic methodologies of use. Like so many (often misunderstood) humanities computing projects, Llull's wheels assert that interpretation is merely aided by mechanism, not produced mathematically or mechanically. That this assertion is sometimes lost on the general academic community is not simply a failure of the devices scholar-technologists produce (although, as my work seeks to demonstrate, we can do a better job of anticipating and incorporating patently interpretive forms of interaction on the part of our users into the systems we create for them). Instead, it displays our failure to articulate the humanistic and hermeneutic basis of our algorithmic work to a lay audience. Further, it reveals the rampant under-appreciation among scholars of the algorithmic nature of an over-familiar machine on which all our work is predicated: the book. ${ }^{23}$

When I began to examine Ramon Llull, I anticipated closing a description of the Ars 
Magna with some examples of how computing humanists might use his wheels to analyze and reconfigure combinatorially the hidden rules and assumptions that drive our own practice. Instead, I am inclined to argue that the best new use for Llull's old machines might be as defamiliarizing devices, modelling — for a larger and often skeptical or indifferent academic community — the application of mechanical or algorithmic systems to problems of interpretation with which scholars engage on a dayto-day basis. A dearth of clear and compelling demonstrations of this applicability to the interests of the academy is the central problem facing the field of humanities computing today. This is the reason our work, like the allegedly "pre-critical" work of bibliographers and textual critics before us, remains insular. ${ }^{24}$

Llull tells us that he chose a graphical and mechanical art partly through inspiration (God revealed the Ars Magna by inscribing with fiery letters the manipulable and discrete leaves of the lentiscus plants on Majorca's highest peak) — and partly out of a recognition that the elements of interpretation should be finite in number, explicit in definition and methodological use, and visually memorable. Seen in this (divine?) light, interpretation lends itself easily to algorithm and apparatus. The enabling devices for scholars described in the case studies that follow are, in their attempt at marrying a similar methodological openness and mechanical clarity to the practice of humanities interpretation, indebted to the work of that "Doctor Illuminatus," Ramon Llull. 


\section{IMAGINARY SOLUTION \#1:}

\section{Dr. Kremlin’s Disc}

\section{Introduction:}

"Dr. Kremlin's Disc" is a multi-player game of specification, association, and interpretation, in which participants cooperate to create and maintain elaborate relational idea-structures. It draws on three ludic traditions that emphasize interpretive flexibility and the free play of allusion but nonetheless require their players to explicate all objects of interest ("primitives" or manipulables) and to express those objects' behaviors and interrelations in near-algorithmic terms. These three ancestors are Ramon Llull's generative Ars Magna, the Glass Bead Game of Hermann Hesse's Das Glasperlenspiel (described by Hipbone creator Charles Cameron as a “game designer's holy grail”) and Peter Suber's emergent "game of amendment," Nomic. Like them, this game is fundamentally cooperative and performative in nature, but nothing in its structure prevents it from evolving in carnivorous ways.

The notion of ludic evolution comes to “Dr. Kremlin’s Disc” from Nomic (described in Chapter III.E below) and, as in that game, the ruleset governing play is open to regular re-interpretation and amendment by players. The "glass bead" connection is realized in the game tokens this document recommends and in the basic mode of play it outlines, which draws on Hesse's allusive ludology. Finally, the debt to Llull is immediately 
evident — not least in the layout of its revolving playing board — but "Dr. Kremlin's Disc" owes its name (along with a few optional end-game pressures) to a terminally shocking device in Marie Corelli’s theosophical triple-decker, The Soul of Lilith (1892):

The singular object that at once caught and fixed the eye in fascinated amazement and something of terror, was a huge Disc, suspended between ceiling and floor by an apparently inextricable mesh and tangle of wires. It was made of some smooth glittering substance like crystal, and seemed from its great height and circumference to occupy nearly the whole of the lofty tower-room. It appeared to be lightly poised and balanced on a long steel rod, — a sort of gigantic needle which hung from the very top of the tower. The entire surface of the Disc was a subdued blaze of light, — light which fluctuated in waves and lines, and zig-zag patterns like a kaleidoscope, as the enormous thing circled round and round, as it did, with a sort of measured motion, and a sustained solemn buzzing sound. Here was the explanation of the mysterious noise that vibrated throughout the house, — it was simply the movement of this round shield-like mass among its wonderful network of rods and wires. Dr. Kremlin called it his "crystal" Disc, — but it was utterly unlike ordinary crystal, for it not only shone with a transparent watery clearness, but possessed the scintillating lustre of a fine diamond cut into numerous prisms, so that El-Râmi shaded his eyes from the flash of it as he stood contemplating it in silence. It swirled round and round steadily; facing it, a large casement window, about the size of half the 
wall, was thrown open to the night, and through this could be seen a myriad sparkling stars. The wind blew in, but not fiercely now, for part of the wrath of the gale was past, - and the wash of the sea on the beach below had exactly the same tone in it as the monotonous hum of the Disc as it moved. At one side of the open window a fine telescope mounted on a high stand, pointed out towards the heavens, - there were numerous other scientific implements in the room, but it was impossible to take much notice of anything but the Disc itself, with its majestic motion and the solemn sound to which it swung. Dr. Kremlin seemed to have almost forgotten El-Râmi's presence, — going up to the window, he sat down on a low bench in the corner, and folding his arms across his breast gazed at his strange invention with a fixed, wondering, and appealing stare.

"How to unravel the meaning — how to decipher the message!" he muttered — "Sphinx of my brain, tell me, is there No answer? Shall the actual offspring of my thought refuse to clear up the riddle I propound? Nay, is it possible the creature should baffle the creator? See! the lines change again — the vibrations are altered, - the circle is ever the circle, but the reflexes differ, - how can one separate or classify them — how?” (Corelli 122-7)

The connection of this crushing Correllian brain-Sphinx to the game will be clarified under "The Kremlin Factor," below. 


\section{Requirements:}

People. This is a multiplayer game. Solitaire is possible, but is not recommended, as it may lead practitioners to an inflated self-opinion. Likewise, odd numbers of players may participate, and (with some obvious rule modifications) in unlimited numbers, but “Dr. Kremlin’s Disc” is optimized for two or four players or teams. Glass Beads. Players need a minimum of 28 small, manipulable tokens, such as marbles or beads — preferably distinguishable in terms of color, size, or shape. Any objects are acceptable, so long as they fit on the device. The Device. The game board or disc itself (as described below). Extra Stuff. Players may find scratch paper and writing utensils helpful. More beads or tokens may be needed for some versions of the game. Game discs made (optionally) of slate call for chalk rather than beads. Other assistive technologies (like six-sided dice) may be called for by player modifications to the ruleset.

\section{The Device:}

The game board, like the famous "Fourth Figure” of Ramon Llull's Ars Brevis, consists of three interlocked rings, each separated into box-like compartments or "camarae" capable of holding game tokens in place. The outer ring, with 16 small camarae, is fixed, but the two inner rings, with 8 and 4 larger camarae respectively, are removable and can rotate freely. At the very center of the ring-structure is a small dish or container (a "pivot") that holds all the game's beads at the outset of play. If gameplay with beads (an aesthetically pleasing but very challenging memoria technica) is not desired, the device can be made of chalkboard, whiteboard, or even paper — any surface 
fit for writing. In this case, box-like compartments are not needed, but the board should still be divided into 28 camarae as specified in the following diagram:

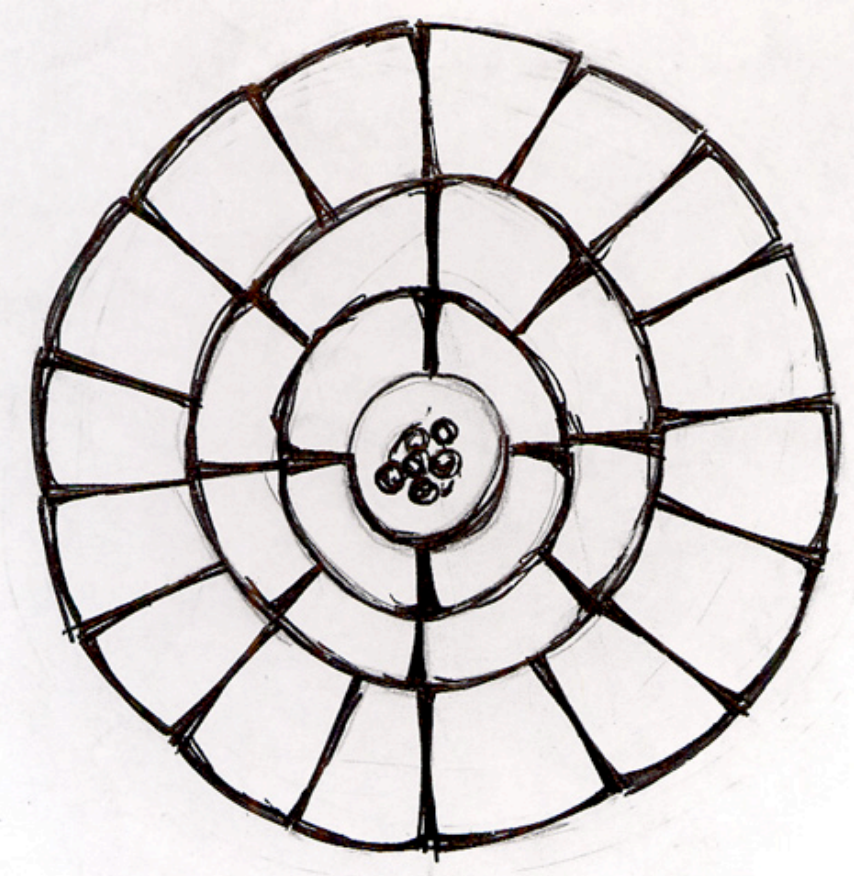

\section{Playing the Game:}

Players predetermine a topic or scope of play (generally some humanistic subject matter they wish to interrogate using this ludic method), or they agree to let that topic emerge in the early course of the game. The central dish or pivot is filled with beads, the two inner wheels are removed from the board, and gameplay proceeds in three stages.

\section{Stage One.}

Each player simultaneously chooses a bead from the pivot, names it for a person, place, 
object, or concept he wishes to have "in play," and then places it in one of the camarae of the outer ring, making sure — in this opening move - that no two beads are adjacent. (Adjacency is to hold special meaning in the game.) Novice players particularly should record the meanings assigned to each bead and may find a graph of the playing board helpful for charting bead positions.

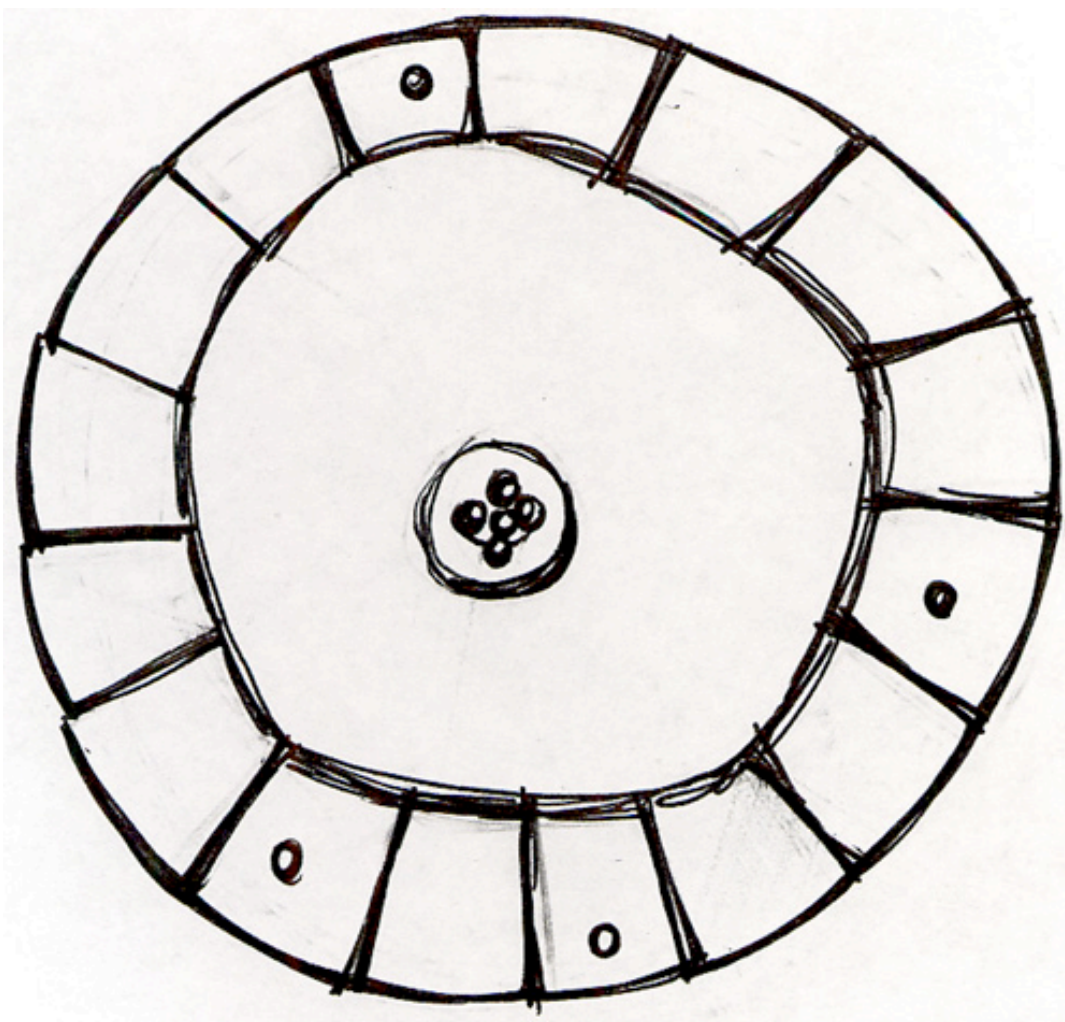

When all players are satisfied that they understand (at least in a general way) the intended semantics of the beads, a joint decision is reached as to syntactic relations among beads that will be deemed acceptable at this stage. A beginner's version of "Dr. Kremlin’s Disc” should limit syntax to adjacency and deem valid any relationship - 
allusive or direct - that a player can articulate to the satisfaction of others. (More complex versions of the game can limit the nature of valid relationships in any number of ways or enable syntactic relations that go beyond mere bead adjacency.) Play then proceeds in turns, with players naming and placing new beads, which can now be adjacent but must follow agreed-upon rules of syntax. In other words, a player may place a new bead ("Helen of Troy”) next to an existing one (“olives”), but only if he can define a relationship between them (perhaps citing H.D.'s poem, in which Helen becomes a Greek marble: "the lustre/ As of olives where she stands/ And the white hands...”).

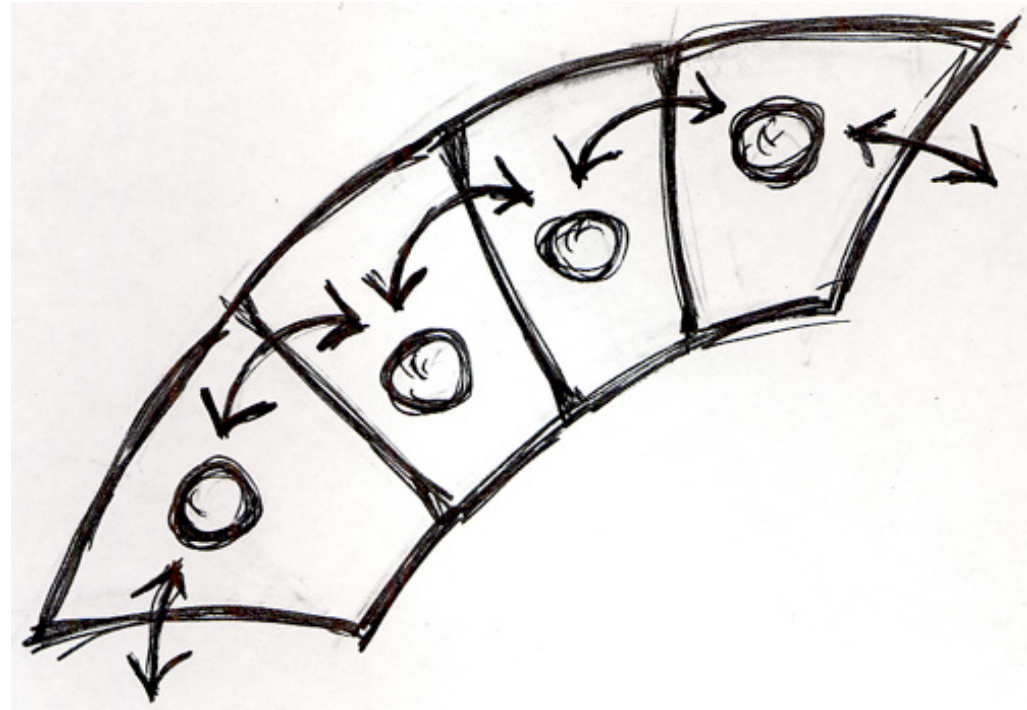

If a bead is placed between two occupied camarae, its relation to the beads on either side must be specified. Play continues until the outer wheel is full. 
Stage Two.

The middle wheel is added to the board and aligned to the outer wheel such that each of its eight camarae encompasses two outer-wheel camarae (now filled with beads).

Players decide whether the same syntactic rules apply as in the outer circle, or if beads must be placed in some new relation here. Play then proceeds in turns, with players placing new beads in relation to each other around the middle ring and to any adjacent beads in corresponding outer camarae.

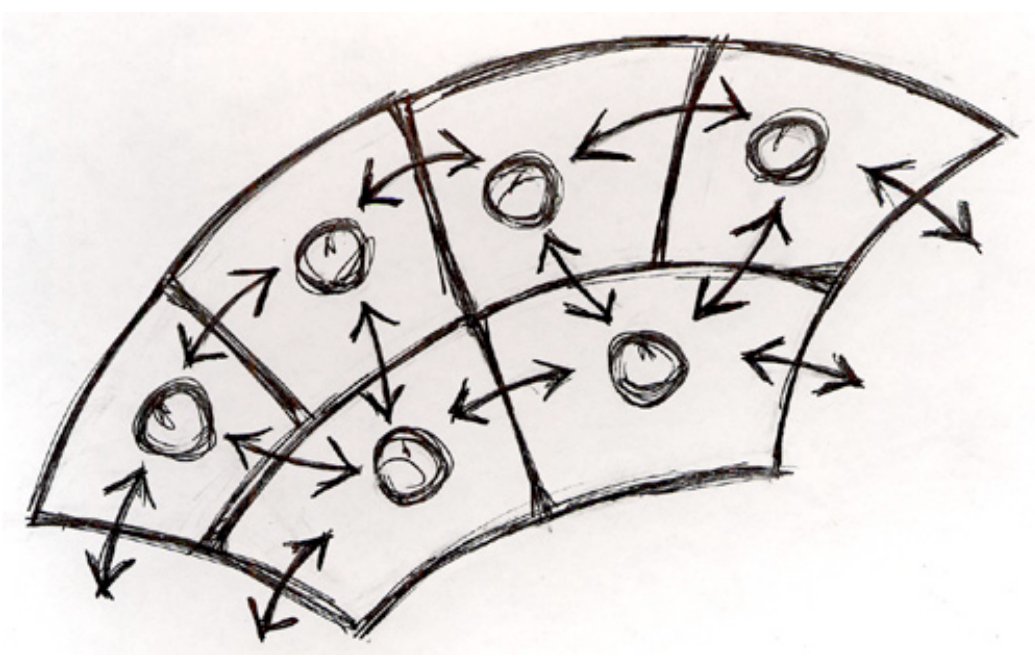

This continues until all camarae in the middle wheel have been filled.

\section{Stage Three.}

The inner wheel is placed on the game board so that each of its four camarae corresponds to two camarae in the middle wheel. Again, players make a determination as to appropriate syntactic rules (perhaps altering a previous formula to change the 
game's level of difficulty, to mirror practices in a specific humanities discipline, or to add constraints they find fruitful in thinking through their game's subject domain). Play proceeds in turn, this time even more tightly constrained, until the inner wheel is filled with beads placed in relation to each other and to beads in the middle wheel.

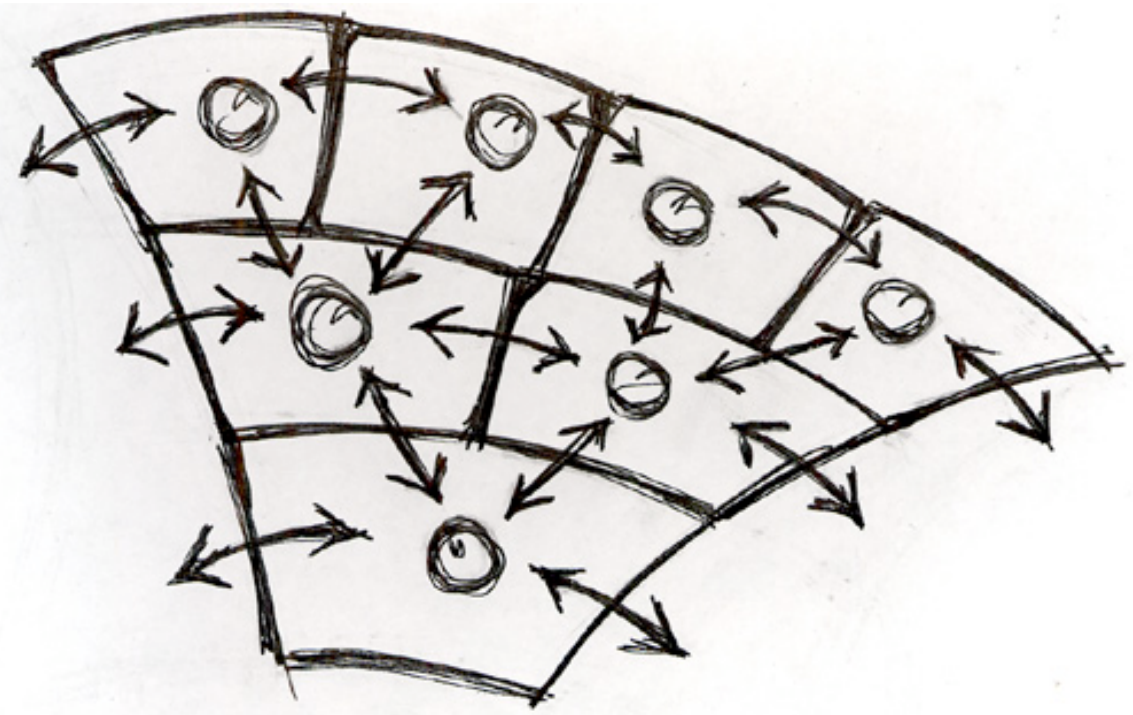

The entire disc is now traced over with a delicate structure of ideas and interactions, and — if players so desire - the game can come to an end. By other standards, however, "Dr. Kremlin’s Disc” has just begun. 


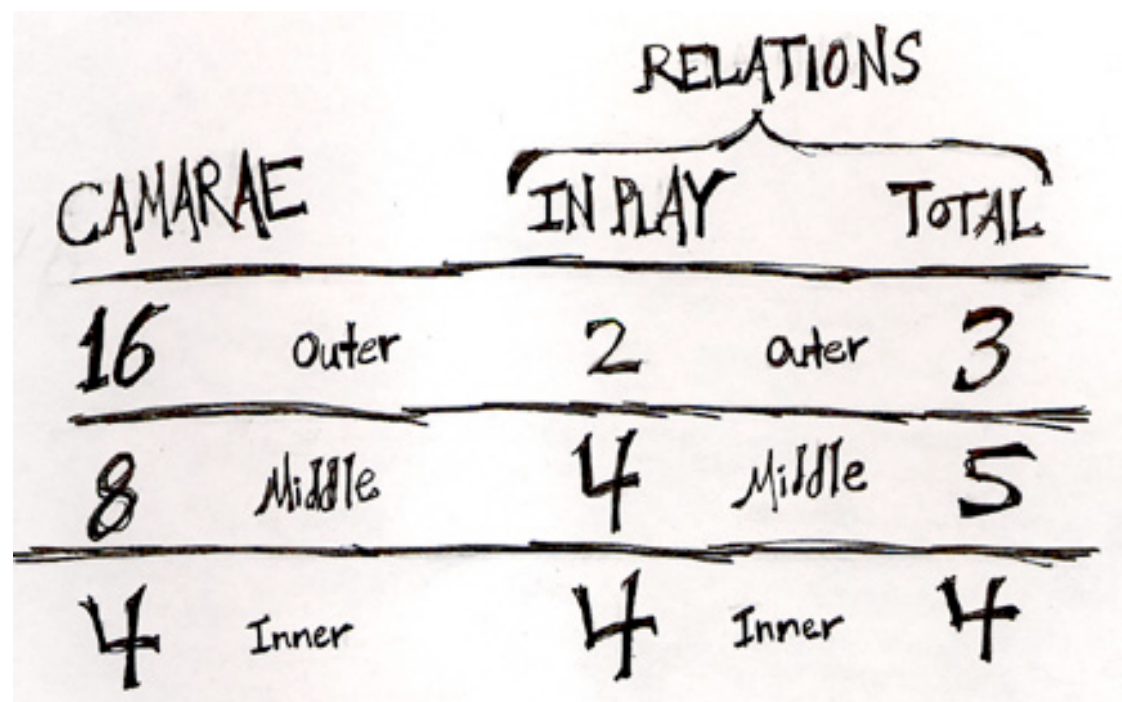

\section{The Kremlin Factor:}

El-Râmi Zaranos is a handsome Egyptian count with an astrally-projecting teenage brother and an undead Gypsy in his London townhouse, — but that's neither here nor there. The important thing, to his old friend Dr. Kremlin, is that he possesses an Elixir of Life. Years ago, El-Râmi helped Kremlin obtain the mysterious mineral of which the ancient scholar's huge, heavy, precariously-balanced disc is made, a mineral that absorbs and reflects starlight in intricate patterns begging elucidation. Kremlin has spent one lifetime analyzing his particular brand of Llullian wheel, and he knows he needs a few more to finish the job. He is therefore delighted when El-Râmi offers him a draught of the elixir, and wakens the next morning with a spring in his step and a healthy appetite, which gratifies his long-suffering German manservant, Karl. Karl has not much longer to suffer, however, because Dr. Kremlin's enthusiasm for disc-interpretation exceeds his 
good sense, and the old man remains in his tower in spite of some sort of tropical monsoon that suddenly batters the Irish coast. In true Corellian overkill, the good doctor is both struck by lightning in the storm and crushed to death by the toppling of his enormous, inscrutable disc.

Unlike Dr. Kremlin, “Dr. Kremlin’s Disc” is designed for extended play. Only a few game extensions are described here, but players are enfranchised to create their own modifications, exploiting the basic affordances of the game's board, its token set, and the ludic algorithm that governs interpretive play.

Some game sessions may profit from particularly Kremlinesque pressures, including: a set time limit for player moves (or for completing each ring of the disc); the incorporation of random events (such lightning strikes as bead swapping or disappearance, dictated by a roll of the dice); or balance-dependent gameplay, in which the camarae of the disk must be filled up in parallel patterns. All of these extensions add the pressure of collapse to “Dr. Kremlin’s Disc.”

Gameplay red in tooth and claw is another option (not necessarily only suited to competitive modes of play) in which players may wish to allow the capture or forced migration of beads according to pre-defined Darwinian laws. Similarly, a game of condensation might hinge on rules for gathering beads together from adjacent camarae under strict conditions of association. And of course, true Llullian games will 
incorporate the spinning of the disc's two inner wheels, together with rules that specify the circumstances and consequences of such an act. What is the purpose of a spin? How should the syntactic connections broken by the spin be handled? For instance, are players responsible for "repairing" them through inventive allusion and reinterpretation of the beads? Are links that break indicative of faulty beads and weak ideas — or do they represent the "exceptions" that shape a "patacritical science? 


\section{Temporal Modelling}

\section{A. Project and Primitives}

Gardner's critique of Ramon Llull's provocative device hinges on a misapprehension of the ends to which it should be applied. The Ars Magna is not, as Gardner assumes, a "logic machine," manipulating concepts as if they were fully-delineated, specified objects and generating universal, incontrovertible truths. If that were the case, his assertion that the varied subject matter on which Llullian wheels act lacks native "analytic structure" and "even agreement on what to regard as the most primitive, 'self-evident' principles” would be perfectly valid (Gardner 18). But few modern users of Llull's system would be inclined to search for absolutes in fields like literary interpretation, historiography, religious studies, or the criticism of art. Pragmatic humanists are more likely to side with William James in a desire to transform theoretical premises into “instruments, not answers to enigmas in which we can rest" (James).

Still, the development of mechanical aids to humanities interpretation requires the establishment of primitives, the basic, domain-specific building blocks or manipulables on which a machine or computer program can operate - even if (as in the case of Llull's alphabetic calculation) the purpose of the operation is to question the aptness or validity of those very primitives and the statements they combine to produce. And Gardner's implied questions deserve to be taken seriously: What does "native analytic structure" 
mean? Is it really lacking in the hermeneutic endeavor as it can be expressed in an enabling mechanism? How can we ever agree on the "most primitive, 'self-evident' principles" at play in a humanities context? How can we avoid, in the construction of interpretive digital environments, designing (or seeming to become) that overly disambiguated "satire of scholasticism" Gardner reviles in Llull?

In the spring of 2001, University of Virginia professor Johanna Drucker and I undertook a project that would test an approach to these problems. I rehearse its history here, before delving into the specifics of its conceptual and practical development and its theoretical and rhetorical goals, because it seems to me a useful example of the way such projects can come into being, and because the scope and limitations of our work were dependent on the material and intellectual pre-conditions outlined below.

Drucker and I were supported in our work by a grant from the Intel Corporation meant to strengthen ties between the computing industry and academia and to allow humanists to address common design problems in an intellectual and creative context removed (and somewhat protected) from the forces that shape the business world. We first came in contact with Intel through conversations with John David Miller, a software architect working in Intel's Architecture Labs (now Intel R\&D), who had, in cooperation with John Maeda of the MIT Media Lab, been at work on a software prototype known as Grand Canyon. Grand Canyon was conceived as an opportunity to 
exploit Intel's nascent 3-D architecture for the personal computer while breaking into a new market based on the growing popularity of digital photography. It was, in essence, an electronic photo album, organized chronologically, which would allow users to place photographs they had taken alongside nostalgic stock images in a three dimensional space (Miller and Maeda). The display mode of the software featured a long canyon or tunnel in which scaffold-like, semi-transparent calendar grids, populated with photos and labeled with dates, were spaced at regular intervals (Figure 1). The user would fly through this VR space, effectively touring and inhabiting a personalized, illustrated timeline.

On review, some problems with the representation were immediately evident. The first was an illogic of organization on the scaffold-like calendar grids. Their familiar structure and graphic intractability seemed to imply a one-to-one connection between locations on the vertical and horizontal axes and specific months and days of the year. Instead, each grid was meant to represent a single year on which images could be placed in random or purely aesthetic arrangements. Even given this broad and loose specification of chronology, the precision of placement of the images within discrete years was somewhat troubling, as it implied a unity of interpretation - a single valid take on "what happened when" — and a lack of concern for duration, causality, and other commonly assumed or applied aspects of temporality and temporal representation. However, as humanists and media scholars, we were most interested in a few promising features of Grand Canyon which struck us as having been perhaps inadequately 
appreciated and explored in its design.

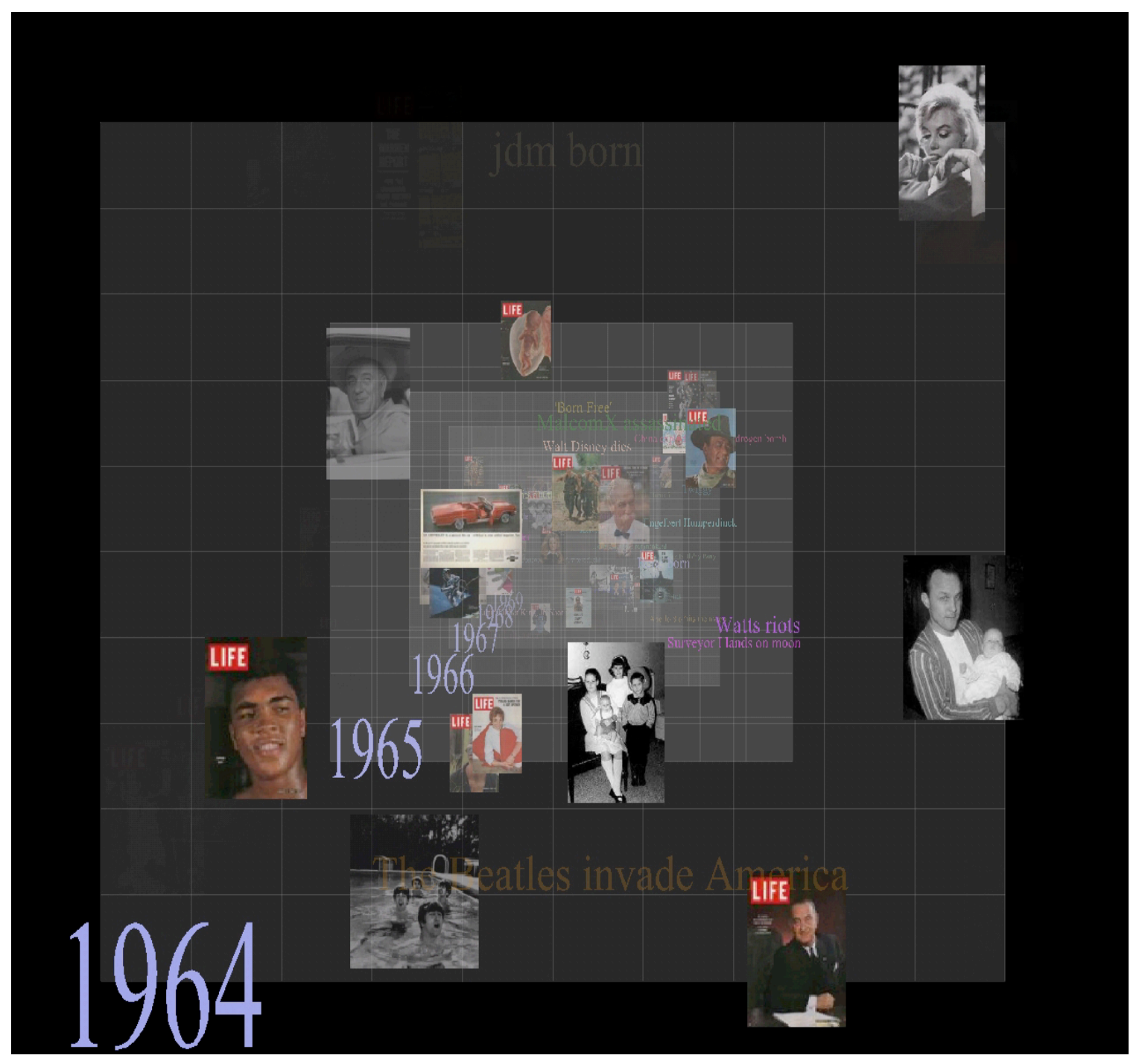

\section{[ Figure 1. Grand Canyon ]}

The first was simple: that its studied inexactitude embodied a diagrammatic approach to time and temporality far removed from the typical domain of the scientific or even the history-textbook timeline, which places great value on a one-to-one relationship 
between event occurrence and symbol placement on a carefully-defined axis. We were confronted here with the visual representation of a new approach to temporality, one to our minds more in keeping with the interpretive interests of humanities scholarship. The other fundamental design features of Grand Canyon were more complex and will be examined here under the categories of subjectivity, structure and flexibility.

The notion of subjectivity as expressed or implied by the practice of building and navigating the timelines seemed to be a critical feature of the software. A user was meant to inhabit the "canyon"-space, moving through some representation of temporality, generally — and specifically in the example we were shown — moving through a chronological representation of his own life and times. The imaginative placement of the viewer inside the timeline seemed to us to open the possibility of active engagement with the visualized environment. In other words, if I am placed within a VR timeline as a semi-active agent rather than — in the (perhaps deceptive) mode more familiar from print - somehow above it as a passive viewer, what, conceptually, is to prevent me from moving beyond mere navigation of the line to the enacting of real changes in its relational structure? And why shouldn't my very presence and the force of my regard exert a kind of quantum influence on the objects that surround me? Grand Canyon made its users into ghosts, passing ethereally through insubstantial arrays. We imagined a different level of engagement with a diagrammatic expression of temporality, and a fuller exploitation of perspectival subjectivity in the digital 
environment.

The flexibility of Grand Canyon as an editing and display environment - in other words, a user's ability to populate and arrange the representation as desired — also caught our attention. In essence, the software permitted its user to develop and embody crude, alternate interpretations of a single span of time. While the visual display as implemented would not accommodate the simultaneous navigation of two competing (or complementary) temporal systems, it was not difficult to imagine how different the experience of motion through the same "canyon" of time could become based on the choices made by users, the designers of the experience. These designers, whether consciously or not, would use the Grand Canyon tool to produce temporally-oriented interpretive representations - individualized models of human experience. As such, we came to see, the lines called for richer construction, for tools and designs that appreciated the subjective and interpretive experience of time, an understanding common to humanities scholarship that time and temporal relations are not uniform, predictable, and uninflected by perception.

Finally, the possibility (expressly facilitated by the digital medium) of extracting interpretive structure from a user-created visual display excited us — for it was evident that the material positioning of images on-screen was reflected in abstract models embedded perhaps inaccessibly somewhere in the software's works. Furthermore, these models, while sharing certain fundamental objects and relations with each other, would 
be of necessity different in each application of Grand Canyon, as users made different choices while building their displays. How much more diverse and revelatory might those structures be, if users were presented with greater freedom of customization and the ability to express subjective inflection? How could we best determine the fundamental temporal metaphors and templates — the Llullian primitives — that such presentation would require? What could we hope to learn from an explicit consideration of information structures extracted from the visual display? How might they be translated and re-purposed in a humanities context? And how much more vital, in the subjective and manipulable environment we were now beginning to imagine, might the interpretive agency of the user in creating temporal models become?

With these questions in mind, beginning in earnest in the summer of 2001 , we undertook research and hosted conversations at the University of Virginia under the rubric of the "Temporal Modelling Project," an ongoing effort which has led to the development of the "PlaySpace" described below, an interactive digital environment for embodying, manipulating, transforming, and analyzing interpretive models of time and temporal relations.

\section{B. Conventions and Contexts}

The first work of the Temporal Modelling Project, geared particularly toward solving the problem of primitives, was to locate and compile a set of core readings in temporal 
representation and its application in a humanities context. Because certain cultural understandings about time and temporality underlie diagrammatic and textual embodiments of temporal relations, we began our work at what Dante Gabriel Rossetti might have recognized as an inner standing point, a subjective prospect embraced with all consciousness of its perspectival limitations and with an intent to exploit them as defining characteristics of a realm of action. ${ }^{25}$ The development of new theories and definitions on which to base our PlaySpace design was therefore not only unnecessary, but also undesirable. Much more effective would be an interpretive modelling environment predicated on traditional understandings of time and temporal relations the assumptions under which humanities documents are produced and explicated. Three core assumptions that framed our work are outlined in an article co-authored with Professor Johanna Drucker of the University of Virginia, which we submitted for publication to Information Design Journal in November of 2001 (reproduced here as Appendix A).

Having assessed the literature on time and temporality, our next step was to assemble a visual archive on which the Temporal Modelling Project could draw during its PlaySpace design phase. We made the scope of this archive manageable by keeping it somewhat book-centered, limited to Western (largely academic) depictions of time. Thus for instance Aboriginal paintings of the Dream Time and the temporal dimension of medieval cathedral architecture did not play a large role in our survey, which was 
meant rather to assess diagrammatic, academic, and rhetorical approaches to time familiar to humanities scholars who might use the Temporal Modelling tool.

The resultant text and image archive facilitated discussion at an Intel-sponsored seminar we hosted in the summer of 2001 , and factored into our emergent goal: the "production of a responsive interface" housing "temporal primitives" (defined as early as September 2001 as a co-dependent "set of objects, actions, and relations") that were "legible, customizable, and sufficiently constrained to generate parameterized output in XML-compatible formats without prior technical knowledge on the part of a user" (Drucker and Nowviskie 1). In other words, we wished to build a visual environment that would allow humanists to model the temporal relations at play in their own fields of study, and to analyze those (perhaps intuitively sketched-out) relations in a rigorous way. This posited environment, first outlined in a software specification and animated storyboard in April and May 2001, became the Temporal Modelling PlaySpace described in depth below. ${ }^{26}$

Invited speakers and participants in the summer seminar at UVA represented the varied interests in temporality of fields such as anthropology, art history, literary interpretation, bibliographical and textual studies, religious studies, history and historiography, information and library science, and the visual and conceptual arts. We presented them with a categorized and annotated packet of images and readings from our literature review. Topics covered in this compilation included conceptual and 
representational issues, informatics and temporality, visualizations, and a set of "themes" related to our field of study: cultural and cross-cultural concepts, geospatial temporality, and narrative theory. In addition to several overviews and abstracts of central goals and concepts, we also included a bibliography and postscript on relativity. ${ }^{27}$ Johanna Drucker's summary of this work (reproduced here in the opening sections of Appendix A) highlights relevant cross-disciplinary scholarship and artistic production and contextualizes our decisions about what we were to call "temporal primitives" - those Llullian constants that would drive our own combinatorial machine. The observation that, throughout our process of research and design, "we were guided by the conviction that our primary user-base for the interface [would] be humanities scholars working [like our seminar participants] from documentary evidence, rather than [from $]$ empirically gathered data" is essential to an understanding of the Temporal Modelling Project (Drucker and Nowviskie 2). In addition, we recognized that "subjective information (detailed annotation and expressive visual forms) might need to be introduced" by scholars into their diagrammatic representations at any point in the modelling process they would undertake using our tool (Drucker and Nowviskie 2).

\section{PlaySpace Design}

The rhetorical scope of our practical work was meant to reach beyond the varied subject domains to which humanities scholars might apply our tool. In essence, the Temporal Modelling PlaySpace we were to develop represents a "time machine" for the discipline 
of humanities computing. Not only does this interactive environment take time and the temporal relations inherent in humanities data as its computational and aesthetic domain, enabling the production and manipulation of elaborate, subjectively-inflected timelines, it also allows its users to intervene in and alter the conventional interpretive sequence of visual thinking in digital humanities.

An overview of traditional ways of thinking about and visualizing time and research on time and temporality informed our design process — primarily the search for and establishment of those "most primitive, 'self-evident' principles" that Martin Gardner's critique suggests are foundational to the reasonable development of any Llullian mechanism (Gardner 18). This section will place that process of research and decisionmaking and its resulting composition and display tools in a humanities computing context, describing the project's practical and rhetorical goals and examining some special visual features for encoding and manipulating subjectivity in temporal relations.

The Temporal Modelling environment we developed embodies a reversal of the increasingly-familiar practice of generating visualizations algorithmically from marked or structured data, data that has already been "modelled" and made to conform to a logical system. Computing humanists certainly take an interest in the visual as a form of algorithmically-generated aesthetic provocation to interpretation (as we have seen in the opening chapter on Llull), but we frequently relegate this activity to the end of humanities computing projects, after the "real work" of content modelling — 
classification, organization, and markup - is complete. Visualization comes into play in the form of graphs and charts generated according to strict, predetermined procedures for knowledge representation from large bodies of pre-organized data. These visualizations, essentially graphical abstractions of patterns embedded in data, enchant scholars with their ability to reveal hidden relationships in information and augment our understanding of encoded material. They are, however, fundamentally static and (as they depend on structured data and defined constraints) predictable, and we are hard-pressed to argue that they instantiate any truly new perspective on the information they reflect. Why, given the fresh possibilities for graphesis the computer affords, should we be content with an after-the-fact analysis of algorithmically-produced representations alone? Temporal Modelling suggests a new ordering of aesthetic provocation, algorithmic process, and hermeneutic understanding in the work of digital humanities, a methodological reversal that makes visualization a procedure rather than a product and integrates interpretation into digitization in a concrete way.

How concrete? The project's composition tools came to enable an intuitive kind of sketching within a loosely constrained but highly defined visual environment delivered through a Web browser using Macromedia Flash MX and open-source server and database software. This sketching forms an integral part of the earliest phases of content modelling, thereby allowing visualization to drive the intellectual work of data organization and interpretation in the context of temporal relations. Aesthetic provocation becomes dynamic, part of a complex dialogue in which the user is required 
to respond to visualizations in kind. Response in kind, that is, in the visual language of the Temporal Modelling toolset, opens up new ways of thinking about digital objects, about the relation of image to information, and about the subjective position of any interpreter within a seemingly logical or analytic system. In fact, it calls for a new definition within the humanities computing discipline of visualization as an activity rather than a result: more responsive, user-created, interpretive, and (like Llull's estimation of his diagrams) both more active and interactive. Visualizations may still result from an algorithmic process, but by locating itself in a responsive composition environment this process has opened itself up to intervention (even co-creation) by a human interpreter in ways unprecedented in digital scholarship.

Our chief innovation in this regard is the translation of user gestures and imageorderings that arise from an iterative dialogue with our composition environment into an accurate and expressive XML representation, which can be exported to other systems, transformed using XSLT, and even employed in the development of a document type definition (DTD) or schema for conventional data-markup practices. The sketching environment in which this rich data capture takes place - the Temporal Modelling PlaySpace — is closely wedded to a theorized (but yet unexecuted) sisterenvironment, the DisplaySpace. There, we hope to provide a set of filters and interactive tools for the manipulation and display of more familiar, algorithmicallygenerated visualizations, derivative either from PlaySpace schemata or the alreadyencoded data structures of established humanities computing projects. Like the 
PlaySpace, though, the projected Temporal Modelling DisplaySpace emphasizes the flux and subjectivity common to both our human perception of time and our facility for interpretation in the humanities. We have not rejected display in favor of the playful engagement our composition environment fosters; instead, we hope to show that a new, procedural understanding of graphic knowledge enhances and even transfigures visualization in the older modes.

\section{Objects, Relations, Actions}

Our work in building the PlaySpace has required a constant articulation of its conceptual distinction from the DisplaySpace (the implementation of which will constitute the next major phase of the Temporal Modelling Project). What quality of appearance or use distinguishes a display environment from an editing environment? At their heart, the mechanisms and processes of the PlaySpace are bound up in:

the positioning of temporal objects (carefully-designed graphical markers representing events, intervals, and points in time) on the axis of a timeline; ${ }^{28}$

the labelling of those objects using text annotation, a user-defined legend explaining color and tone, and a variety of other visual qualities (size, alpha value, etc);

the relation of objects to specific temporal granularities (the standards by which 
we mark hours, seasons, aeons — represented in our PlaySpace by tic-marks which open themselves to user-adjustment and automatic calendrical calculations);

and, in complex interaction, the interrelation of objects, as expressed both graphically on the PlaySpace stage and in the underlying temporal data model.

Each of these interpretive actions - the specification of objects and orderings, their explication and interrelation — additionally involves a practice we designate inflection. Inflection is the graphic manifestation of subjective and interpretive positioning toward a temporal object or (in a sometimes startling display of warping and adjustment) toward an entire region of time. This positioning can be on the part of the prime interpreter, the user of the PlaySpace, or inflections can be employed to represent and theorize external subjectivities: the inferred interpretive standpoint of a character in a work of fiction, for instance, or of an historical figure, movement, or Zeitgeist.

The energies of the PlaySpace are all focused on enabling understanding through iterative visual construction in an editing environment that implies infinite visual breadth and depth. In contrast, the proposed DisplaySpace channels energy into iterative visual reflection by providing a responsive, richly-layered surface in which subjectivity and inflection in temporal relations are not fashioned but may be reconfigured. 
The "conceptual primitives," or "least common denominators" of our temporal schema, are detailed in Appendix A below (Nowviskie 2003, p.5; cf. Drucker and Nowviskie: Appendix A: "Conceptual Primitives"). My own working notes from a conversation with Johanna Drucker and Worthy Martin, in which I proposed that we view Temporal Modelling software in terms of a table of objects, relations, and actions, are presented in Figure 2 below (see also note to Figure 2). Subsequent work at SpecLab and with the newly-formed University of Virginia group for Applied Research in 'Pataphysics (ARP) has suggested the utility of these three basic categories for conceptualizing the requirements of humanities software. When we think of humanities computing design problems in terms of relevant objects, actions, and relations, we tend to balance our attention fruitfully between the intrinsic qualities of the documents and materials under scholarly analysis and the established practices and new opportunities we wish to support in a digital environment. Furthermore, when all of these elements join in humanities computing software that reflects common practices and affordances of a humanistic understanding of temporality and permits — as freely as possible within a fruitfully constrained editing environment — basic scholarly activities of inflection, annotation, and manipulation, we can achieve a kind of Llullian openness of interpretation to algorithmic autopoiesis. Like Llull's alphabetic constants, Temporal Modelling "objects" need not imply an untoward "objectivity" about the subjects and structures they depict. Instead, the identities of our objects (such as temporal points, events, and intervals) are fluid and determined as much as possible by the users of our system, rather than by the system itself. 


\section{[ Figure 2. Draft Table of “Conceptual Primitives"]}

(Nowviskie and Drucker, with input from Worthy Martin; ca. 11 September 2001) ${ }^{29}$

\section{OBJECTS or ELEMENTS:}

points, events, intervals

temporal axis (timeline)

temporal grid (calendar)

temporal dial (clock face)

granularity markers

metrics (intrinsic and extrinsic)

start and stop points

"now" and the nowslider

\section{RELATIONS or STRUCTURES:}

order and ruptured order

the "dividing instant"

multiple and/or inflected granularities

visual positioning of elements "certainty" of temporal position

"certainty" of boundedness

alternative iterations (nowslider)

degrees of inflection and relation

\section{ACTIONS or OPERATIONS:}

generate/view "slices" of time

position/label elements

order and reorder elements

“inflect" elements

attach and detatch metrics

choose, inflect, and zoom metrics

define intrinsic granularities

"nowsliding"

\section{Inflecting Temporal Relations}

Temporal Modelling inflections (such as causality indicators, notation for literary foreshadowing, or markers for "special" events) were originally classified into two groups, contingent on the degree of their relation to - and interrelation with - userdefined objects in the PlaySpace. What we came to call "semantic" or "vocabulary" and "syntactic" or "grammar" inflections are discussed in Appendix A (subheading: “Subjective Inflections”). 
Semantic inflections are considerably easier to define and implement. Syntactic inflections have proven a good test of our model's robustness. As we entered the implementation phase for syntactic inflections in the autumn of 2003, my conversations with Johanna Drucker and with Jim Allman of Interrobang Digital Media, a programmer and designer who has assisted on the project from its early stages, led to a new conception of inflection, which may take shape in future implementation phases. The new approach results in a broadening of the original scope for inflections, which itself emerged largely from our review of narratological and discourse theory and evolved in response to requests by Temporal Modelling seminar participants and potential users at UVA's two humanities computing hubs, the Institute for Advanced Technology in the Humanities and Virginia Center for Digital History. It is attractive to the project chiefly because it meshes well with our goal of enabling iterative sketching and user-defined visualization techniques as an aid to humanities interpretation — but it also forms a compelling addition to the Temporal Modelling toolset because of its relation to another effort at melding history with graphesis and the hermeneutic with the algorithmic method: Michael Leyton’s work on process grammar.

Leyton's grammar posits that any shape may be expressed mathematically in terms of six phase transitions, roughly corresponding to a squashing action, continuing to the point of indentation into the body of the object and ultimately bifurcating into a bay, and 
internal resistance, continuing to the point of protrusion outward from the body of the object, which ultimately bifurcates into a lobe. The real purpose of the grammar is not to specify actions to be performed on an object, but rather to infer the metamorphic history of any object from its present shape. Using the predictive equations these phase transitions imply, Leyton historicizes the visual artifact: "a shape is simply a single state, a frozen moment, a step outside the flow of time; and yet we are able to use it as a window into the past” (Leyton, Process Grammar).

Users of the Temporal Modelling PlaySpace likewise want to create windows - not into the past per se, but into interpretive representations of the past (or of the future or present). We therefore propose to apply the process grammar concept not as an archaeological or revelatory tool but rather as an artistic, constructive one. This is in tune with our notion of visualization as active and procedural. PlaySpace visualization tools are not meant to show hidden patterns in data, but rather, as employed by a human interpreter, to co-create them. In fact, scholars working to create temporal models in the PlaySpace may wish to design shapes that seem to be the products of forces acting over time, that embed history and subjective response to history. Where Leyton figures his process grammar as a deductive rule-system for deriving history from the visual, our syntactic inflections embody a constructive set of rules for implying historical states and changes through visualization.

To that end, a next step in the implementation of the PlaySpace will involve a shift from 
exclusive dependence on prescribed, pre-defined syntactic inflections (such as "foreshadowing" and "causality"), which participate perhaps too exclusively in certain interpretive disciplines, to the application of a broadly-applicable structural "palette of possible effects" (Drucker, personal communication 2003). In simple terms, we hope to design a flexible set of tools whose generic graphical consequences as enacted on temporal objects — such as stretching, merging, morphing, linking, verging, surrounding, displacing — can be labeled freely by our users, much in the same way that the PlaySpace currently supports user-defined color legends in model-saving and display.

\section{Digital Modelling as Interpretive Practice}

\section{Modelling (in) the PlaySpace}

This description has focused on specific qualities and tools of the Temporal Modelling Project, especially as they relate to the embeddedness of subjectivity, uncertainty, and interpretation in every act of representation. Our very process of design selfconsciously embodied this orientation toward information and software engineering. We made every effort to work from imagery as much as from ontology, coupling our research efforts in the philosophy and data-driven classification of temporal relations with the intuitive and experimental work of artists, students, and scholars of whom we asked questions such as: "What does a slow day look like?" or "How might you paint anticipation or regret?” As our underlying architecture became more stable and we began to assemble a preliminary notation system for temporal objects and inflections, 
we made a practice of asking of each sketch we floated, "What does this imply?" and "What relationships might it express?" No visual impulse was dismissed out of hand; instead, we retained all evocative images, frequently finding use for them later, when our iterative process of development had revealed more about their implications in context.

In this way, the necessity of a special feature of the Temporal Modelling Project was impressed on us: its capacity for expansion and adjustment. The objects, actions, and relations defined by our schemata and programming are not married inextricably with particular graphics and on-screen animations or display modes. Just as we have provided tools for captioning and coloring (and the ability to regularize custom-made systems with legends and labels), we also seek to make possible the upload and substitution of user-made standard vector (SVG) graphics for the generic notation systems we've devised. This is more than mere window-dressing. Our methodological emphasis on the importance of visual understanding allows the substitution of a single set of graphics (representing inflections for, say, mood or foreshadowing) to alter radically the statements made possible by Temporal Modelling's loose grammar. Users are invited to intervene in the interpretive processes enabled by our environment almost at its root level.

A similar sensibility governs the output of a session in the PlaySpace environment. PlaySpace visualizations consist of objects and inflections in relation to each other and 
(optionally) to the metric of one or more temporal axes. The editing process

involves the placement and manipulation of these graphics on a series of user-generated, transparent layers, which enable groupings and operations on groups (such as zooms, granularity adjustments, panning and positioning, simple overlays, and changes in intensity or alpha-value) meant to enhance experimentation and iterative information design inside the visual field. When the user is satisfied that a particular on-screen configuration represents an understanding of his data worth preserving, he may elect to save his work as a model. This means that the PlaySpace will remember both the positioning of graphic notations on-screen and the underlying data model - in the form of an XML schema - that these positions express (Figures $3 \mathrm{a}$ and b).

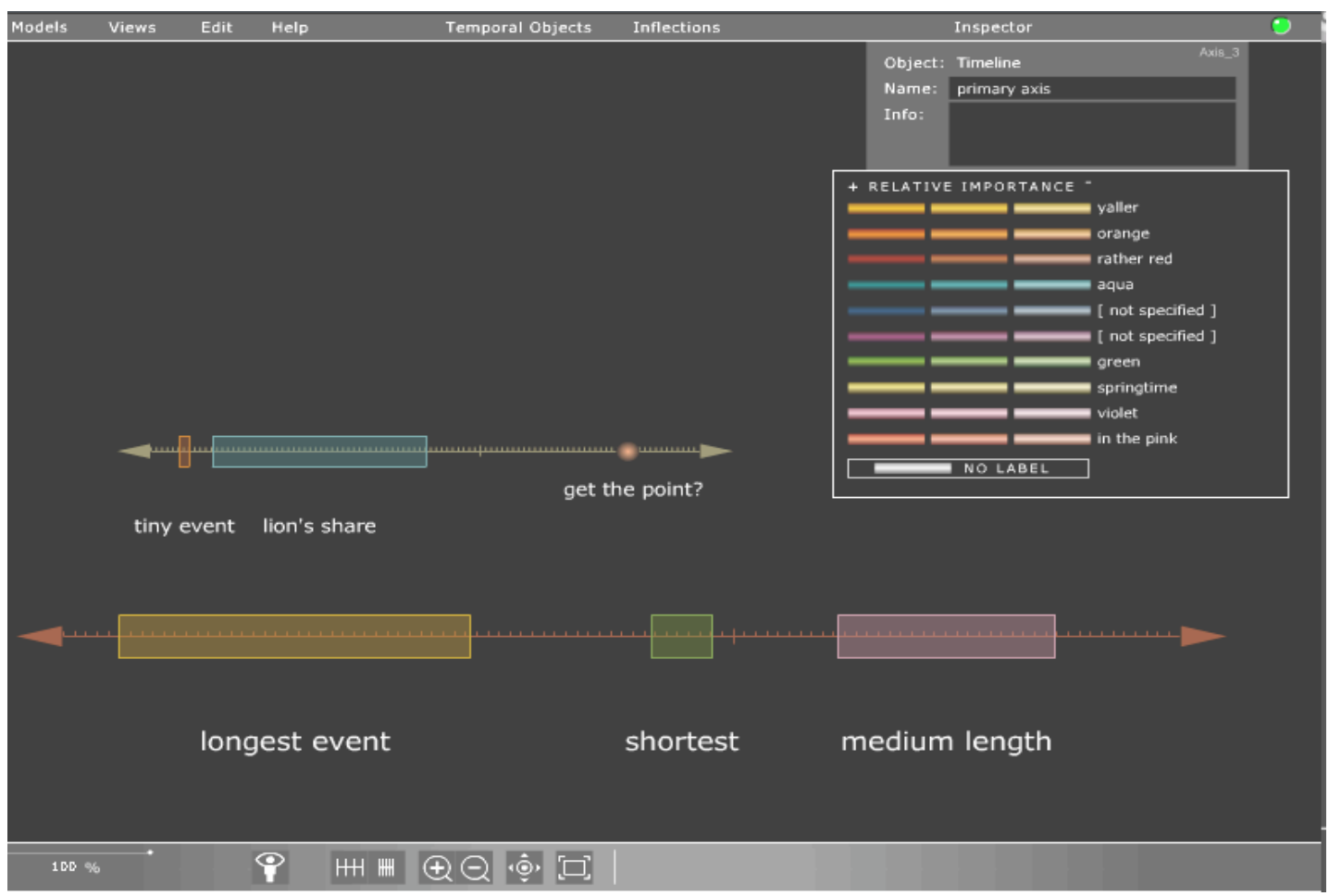

[ Figure 3a: Simple PlaySpace View ] 


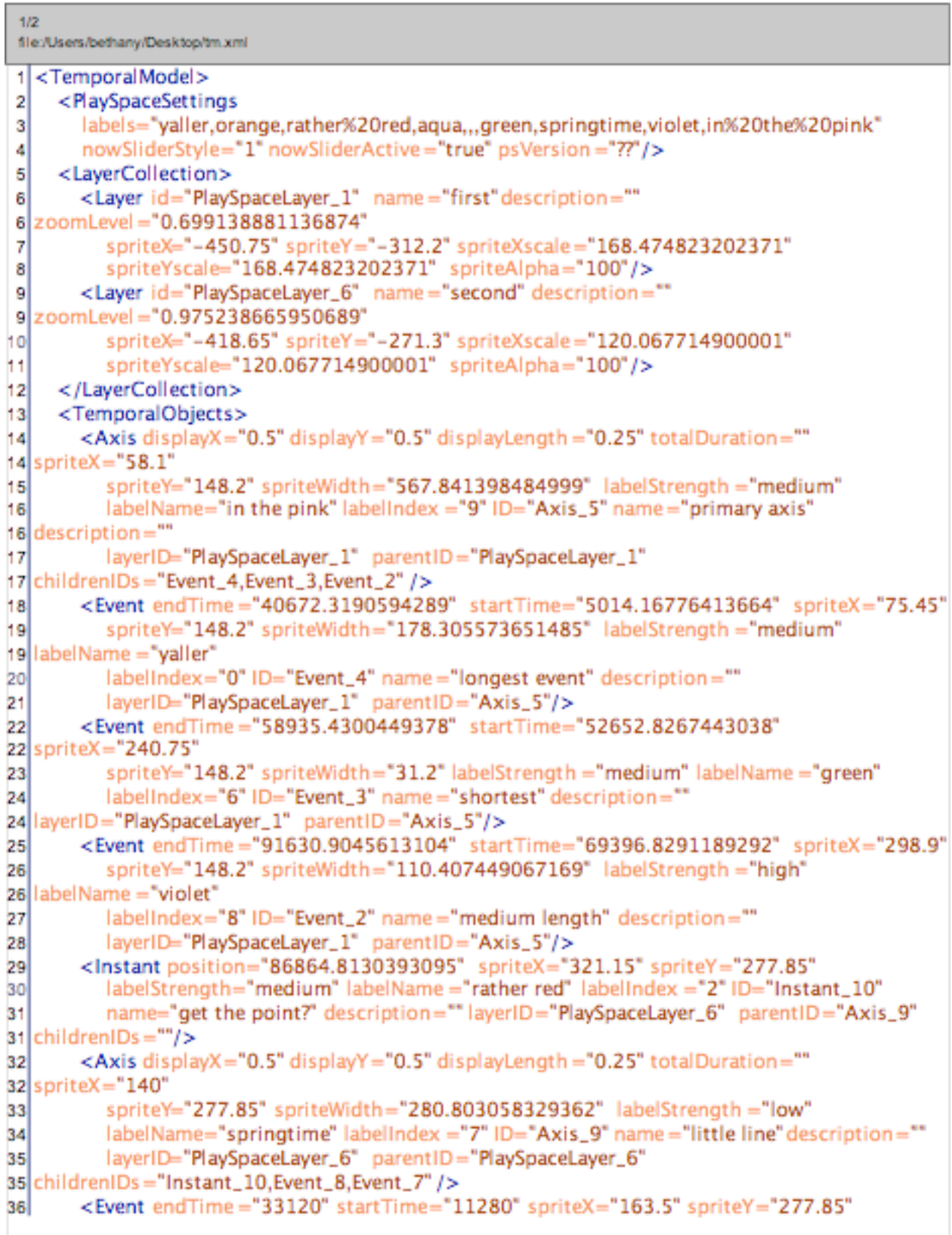

\section{[ Figure 3b: Corresponding Data Model ]}


This data model can then be exported and used elsewhere or even edited outside the PlaySpace and uploaded again for visual application. Most interesting is the way in which transparent editing layers function in the definition of PlaySpace models. The process of saving a model requires that the user explicitly identify those layers belonging to a particular, nameable interpretation of his material. This means that a single PlaySpace session (which can support the creation of as many layers as hardware limitations make feasible) might embed dozens of different visually-expressed interpretive models: some of which are radical departures from a norm; some of which differ from each other by a small, yet significant, margin; and some of which are old friends, imported into the PlaySpace from past sessions, from collections of instructional models representing conventional understandings of history or fiction, or from the efforts of colleagues working in collaboration on research problems in time and temporal relations.

Our software — and its underlying vision for tool-building in humanities computing — defines a model quite simply as an interpretive expression of a particular dataset. More importantly, it is what the interpreter (the user of our composition tool) says it is at any given point in time — not a schema dictated wholly from above, a priori to the actions of the end-user, hard-wired into the system. Exclusive use of the latter sort of modelling in digital archives and collections is perhaps justly reviled by some humanists suggesting to their better angels that computerized models are alien things: if not alien to imaginative practice as such, 
then certainly alien to the received inheritance of literature and art... In this community of readers, the very idea of a "standard generalized markup," which is to say a standard generalized interpretation, is either problematic or preposterous. (McGann, Radiant Textuality 139-40)

We find the flexibility inherent in our Temporal Modelling mode of operation akin to the intuitive and analytical work of the traditional humanities at its best. ${ }^{30}$

\section{Diagrammatic Reasoning}

The Temporal Modelling Project was in part undertaken in order to challenge a commonplace "long-standing prejudice against diagrammatic representation" in the academy (Shin and Lemon 1). "Sentential representation systems (e.g. first-order logic) have been dominant" in philosophy, logic, and cognition, and in trickle-down models and theories in other humanities disciplines, in part due to a feeling that diagrams are too rhetorical a mode — inherently misleading and suited only to heuristic ends (Shin and Lemon 1). Diagrams may be used to explain a concept or articulate a proof, but they are rarely seen as an integral part of the thinking and exploratory process. Diagrams, as typically employed, are purely illustrative.

But new interest in multi-modal reasoning has begun to rehabilitate the diagram as a tool not only for expressing interpretive subsets of relational information but also for thinking through and modelling complex systems. (This is of course no new idea. We have already seen how Ramon Llull represented complex statements and worked 
through interpretive problems diagrammatically in the thirteenth century, affording through a series of graphs and tables the "basis for calculation in accordance with prescribed rules" (Bonner 65).)

However, B. Chandrasekaran, co-editor of the standard textbook on diagrammatic reasoning, credits current research in cognitive neuroscience with bringing issues of representation to the forefront of computational and logical systems-building. "Antirepresentationalism," Chandrasekaran writes, "is not a practical option any longer: information and representation are an inevitable part of the needed vocabulary" for examining the manner in which agents process, store, and reason about abstract and concrete problems (2). To this end, some fundamental clarity is required about our definitions of representation and reasoning, and about the distinctions between visual, spatial, and diagrammatic representation.

Ambiguity has crept into the cog-sci discourse on visual and spatial systems at its most basic level. Glasgow makes a clear distinction between the visual as purely instrumental - uninterpreted images which come to their viewers like scattered pixels on a screen — and the spatial as such: visually-acquired images interpreted in terms of their indicated spatial or topological relations (Glasgow \& Papadias). Others, however, apply the term visual to "representations, interpreted or not, rising from the visual modality," while spatial denotes "knowledge of space, its occupants and their mutual spatial relations, leaving open [the] modality or modalities — visual, auditor [sic], 
kinesthetic or haptic - that supplied the information” (Chandrasekaran 3).

Chandrasekaran further applies attributes of color and texture as part of the exclusive "representational repertoire" of the visual (they are absent from spatial representation), while motion is figured as a potential quality of both modes.

The concept of diagrammmatic representation elicts more general agreement. A diagram is defined as a "form of spatial representation, explicitly constructed and intended to be visually processed, containing elements that have a conventional semantics, displaying the spatial relations among the elements" (Chandrasekaran 3). These elements commonly exhibit visual properties, such as color or texture, that extend the "representational vocabulary" through which they are meant to be interpreted. Furthermore, some elements of diagrams, like labels or annotations, are meant to stand outside of or above the spatialized field and do not constitute part of the representation. In these cases, "conventionality in element semantics provides clues to the consumer of the representation about which aspects of the diagram are to be taken seriously and which are to be taken as incidental" to the fundamental statements being made (Chandrasekaran 3). A 1997 session of the NSF Workshop on Visual Cognition and Decision-Making in the Spatial Domain proposes a more basic definition: diagrams are abstractions, meaning that they extract and summarize a selective subset of information and represent (along with their "content") the rhetorical and interpretive decisions of their creators (Iwasaki). 
This definition betrays a lasting, Platonic tension in the field of diagrammatic reasoning. As rhetorical constructs, must diagrams always lie? Recent independent work by logicians Shin and Hammer seeks to demonstrate that there is "no intrinsic difference between symbolic [that is, first-order sentential] and diagrammatic systems as far as their logical status goes" (Shin 1994; Hammer 1995; Shin \& Lemon 1). The Stanford Encyclopedia of Philosophy entry on diagrams presents a history of the articulation of diagrammatic set systems from Euler to Venn with later extensions by Peirce and Shin in order to show how "simple intuition about diagramming syllogistic reasoning has gradually been developed into a formal representational system” and to illustrate an "interesting tension and trade-off between the expressive power and visual clarity of diagrammatic systems," all of which tends toward the proof that diagrams and graphical systems such as Peirce’s Existential Graphs are logically “equivalent to a predicate language” (2). Ultimately, Barwise and Etchemendy, developers of the multimodal Hyperproof system, conclude that "there is no principled distinction between inference formalisms that use text and those that use diagrams. One can have rigorous, logically sound (and complete) formal systems based on diagrams” (1995; 214).

But perhaps we might not want diagrams to marry themselves so closely to formal logic that they become divorced from human eloquence. (If they are not to lie, perhaps after Huck Finn — they could still help us tell a constructive "stretcher," or show us when we're stretching.) The history of critique and extension of diagrammatic systems is directly related to problems of expressivity and ambiguity — those primary fields of 
expertise of humanists from a variety of disciplines. Venn's criticism of Euler's illustrative system for syllogistic representation (Euler Circles) hinged on his identification of "the weak point in this and all similar schemes:" that they depict "in strictness the actual relation of classes to each other, rather than the imperfect knowledge of these relations which we may possess or may wish to convey" (Venn 510). Similarly, Peirce critiqued Venn's diagrams (a modification of Euler), by pointing out that they have "no way of representing the following kinds of information: existential statements, disjunctive information, probabilities, and relations” (Shin \& Lemon 2.3). Peirce's resulting diagrams increased the expressivity of Venn, but at the cost of more complexity - effectively defeating that great advantage of the diagrammatic statement identified by Barwise and Shimojima: that it constitutes a kind of cognitive "free ride," in which a conclusion or inference "seems to be read off automatically from the representation of premises" (Barwise \& Shimojima 1995; Shin \& Lemon 4.3). A later revision by Shin re-injects some of Venn's original visual clarity (by means of shading) into Peirce's system for disjunction and existential expression (Shin 48ff).

Shin's other major contribution is the presentation of the diagrammatic as a formal representation system equipped with its own syntax and semantics. The syntax tells us which diagrams are acceptable, that is, which are well-formed, and which manipulations are permissible in each system. The semantics defines logical consequences among diagrams. Using these tools, it is proven that the systems are sound and complete, in the same sense that some symbolic logics 
are. (Shin \& Lemon 2.4; Shin 48ff.)

My own explicit definition of some diagrammatic elements of the Temporal Modelling interface as "syntactic" and some as "semantic" came about as an intuitive, descriptive move in the development of our inflection system. This is just, perhaps, another example of our approach to the tool-building exercise as humanistic, but it is interesting to note that Shin and Lemon identify a related trend in diagrammatic work, posing a "fundamental challenge to some of the assumptions held about representation systems." The traditional application of some critical concepts such as "syntax, semantics, inference, logical consequence, validity, and completeness to sentential representation systems only" has been challenged by this new approach. It concludes that, "for any representation system, whether it is sentential or diagrammatic, we can discuss two levels, a syntactic and a semantic level” (Shin \& Lemon 2.4).

The presence of a syntactic and semantic level in any diagram leads us naturally to the notion of diagrammatic reasoning. Chandrasekaran points out that "reasoning" is too often used as "a generic equivalent of computation," meaning that we speak of computer programs "reasoning" about geometric or spatial relations when in fact they are simply performing computations on geometric and spatial information. A "narrower use" of the term is more appropriate to diagrammatic reasoning:

In this sense of reasoning, the agent starts with some given assertions, and makes additional assertions as inferences, using rules of inference. For example, when Barwise and Etchemendy speak of diagrammatic reasoning, they intend for the 
diagrams to be assertions of some facts or hypotheses, and the goal is to arrive at inferences that satisfy the standard notions of valid inference. (Chandrasekaran 4).

She further identifies three distinct types of diagrammatic reasoning, all of which bear on our notion of temporal modelling and contribute to the kind of work we wish to enable on the part of our users. The types include predicate extraction and projection, the use of diagrams in simulation, and spatial reasoning.

The extraction of predicates from spatial information is a formalized activity akin to the data relations we proposed for our Temporal Modelling PlaySpace from its outset — its (somewhat illusory) ability to "extract" a data model in well-formed XML from the onscreen sketches or visualizations a user creates using our paintbox-like set of widgets. ${ }^{31}$ Predicate extraction depends on a commonplace about spatial representation: given a diagrammatic image, a set of "open-ended and domain-and task-dependent" corresponding facts can be asserted, facts which are "computable from the spatial information in the representation" (Chandrasekaran 4.1).

Predicate projection, on the other hand, is a fascinating complement to this process, the future applicability of which to Temporal Modelling is immediately evident: "given a set of spatial predicates pertaining to a situation, this... process creates a spatial or diagrammatic representation, or modifies an existing one, so as to match the assertions" made in sentential form, externally to any visualization (Chandrasekaran 4.1; Davis ch. 
6). In effect, this notion extends our present use of the XML translations stored in our data library when users create and save models. Currently, we allow users to work with visualizations and their complementary data structures in isolation or concurrently, driving representation in both modes intentionally, as solitary agents in the interpretive process. Predicate projection allows us to imagine the intervention of a machine interlocutor in this process (similar to that emerging from thought experiments about a current SpecLab project, the 'Patacritical Demon). ${ }^{32}$ What if the PlaySpace began to play along, by actively intervening in the user's production of diagrams? This particular "demon" could, behind the scenes, run a predicate projection process which allowed it to respond to the assumptions and relations embedded in a user's diagram, by extending the representation (in "constructive" or appropriately deformative ways) or by creating new diagrams which could serve as a form of aesthetic provocation. In effect, it would say: "Given the visualization you've created, I make the following set of assumptions, which I then use to generate this temporal diagram - an hypothesis — in response.” What new insights might emerge?

Simulation is a mode of diagrammatic reasoning closely related to predicate extraction. Here, programs or cognitive models "do not reason in the sense of moving from cognitive state to cognitive state by processing truth-values of assertions. Instead they make use of simulation to generate the next state" (Chandrasekaran 4.3). These simulations have both predictive and (like Leyton's process grammar discussed in section C3 above) archaeological value: they compute spatial relations in stages 
according to a set of proposed actions and interactions - the rules or algorithms which govern change. The means by which diagrammatic simulation is accomplished in a computational environment (usually with the use of an array model) are considerably less constrained than in current models of human spatial information processing. Cognitive scientists participate in the longstanding debate on the nature of imagery by proposing that internal "analog" processes guide our ability to reason spatially — we think in pictures, and imagine manipulating them in the same way real-world objects can be manipulated. The leading alternate proposal in fact models human spatial cognition in terms of predicate extraction and projection (Chandrasekaran 4.3).

Finally, spatial reasoning comes to us meaning little more than the "making [of] trustworthy inferences with spatial and diagrammatic representations," in which an interpretive agent (human or machine) behaves toward a diagram in the same way, using the same inferential processes, it would if reasoning about a sentential statement or other model (Shin \& Lemon 3). This is certainly not to say that the objects of spatial reasoning have no particular limitations or affordances that bear out special treatment.

Shin and Lemon speak of these as the "consequences of spatial properties of diagrams" (Stanford 3). As an example, they point out (with Bertrand Russell) that spatial relations between objects in a diagram can be used to represent relations between objects in some other domain. Sequential languages (e.g., symbolic logics, natural languages), however, use only the relation of concatenation to 
represent relations between objects. (Russell 1934; Shin \& Lemon 3)

The question, as they pose it, is "how well can spatial relations and objects represent other (possibly more abstract) objects and relations?” Diagrammatic spatial relations may be "direct and intuitive," but they too have their "perils" - most notably in the constraints leveled on them by their common planar mode of representation (Shin \& Lemon 3).

One such example of topological and geometric limitations infringing on diagrammatic expressivity comes to us from graph theory, in which it is well known that some quite simple structures cannot be represented in a plane. A case in point is the conventional non-planar graph known as K5, which consists of five nodes, each of which is connected to the others by arcs. None of the arcs should cross, and this small constraint makes K5 impossible to draw in two dimensions. (It is therefore not reproduced in these pages!)

Shimojima’s “constraint hypothesis" helps define the problem:

Representations are objects in the world, and as such they obey certain structural constraints that govern their possible formation. The variance in inferential potential of different modes of representation is largely attributable to different ways in which these structural constraints on representations match with the constraints on targets of representation. (Shimojima 1996a)

In other words, constraints on diagrammatic expressivity remind us that "spatial representations are not of general quantified propositions, but of concrete instances," 
and as such they "often provide a convenient representation for models of propositions" (Chandrasekaran 4.2).

We too identify the inherent spatial tension in diagrammatic representation and modelmaking as fruitful: users of the Temporal Modelling PlaySpace are invited to work within a system of visual and conceptual constraint in order to interrogate their own models more closely. Our digital environment itself works along lines Chandrasekaran has identified as "useful" — in which a "cognitive state is represented as having two components, a spatial one and a corresponding predicate representation, with the two components matching each other" (4.2). The PlaySpace composition tool, with its open stage for visualization, is matched in our project with a "corresponding predicate representation" in the form of XML models stored in the Data Library.

This arrangement is the very definition of "multi-modal" representation, the concept with which I opened our discussion of diagrammatic reasoning. Multi-modal representation, in which correlated information exists simultaneously in perceptual and conceptual modes, is generally agreed not only to match natural cognitive processes most closely, but to result in the production of the most effective diagrams: Representational changes in one mode typically give rise to corresponding changes in other modalities, giving the multi-modal representation some degree of inter-modal coherence. Problem-solving is driven by the most relevant information in any domain. The reasoning might be driven by spatial predicates 
at one moment and by conceptual predicates at the next. Each move forward results in a new state and in updating of representations in all modalities. (Chandrasekaran 5)

The work of Lemon et al. further demonstrates that once intuitive notions about the efficacy of diagrams can now be evaluated in terms of the "standard formal properties of languages" (Lemon 1999).

But it is worth noting - especially in the humanities context in which we have deliberately situated our electronic environment — that so-called "efficacy" in representation must not necessarily be a major, structuring goal. After all, the Temporal Modelling Project is intended to serve humanists who may wish to represent qualities in their models that are as evocative and ephemeral as the documentary sources from which they are drawn. And removing all potentially-misleading "rhetorical" components from our diagrammatic system may be to remove the system wholly from aesthetic and pedagogical application as we have imagined it.

Fortunately, we come to diagrammatic reasoning at a point in its long history when the notion of efficacy is still very much up in the air. Some recent work, for instance, makes an argument for the deliberate retention of "vagueness" in diagramming, demonstrating that this quality can be exploited for effective problem-solving (Goel 1995). And even Shin and Lemon, staunch defenders of the logical equivalency of diagrams with traditional linear proof calculi, observe that effective limitations of diagrams resulting in 
our "not being able to represent contradictions" could generate "interesting insights into the nature of diagrammatic representation:"

If a central goal of language is to represent the world or a state of affairs, then representing contradictions or tautologies is called into question. Neither contradictions nor tautologies are part of the world. How can we draw a picture, or take a picture, of the contradiction that "it is raining and it is not raining?" How about the picture of the disjunctive information "it is either raining or not raining?” Now, we seem to be much closer to Wittgenstein's classic picture theory of language. (Shin \& Lemon 3.2)

Of course, our own modelling project wishes to assert — through a similar application of diagramming and visualization — that contradictions and tautologies are absolutely "part of the world," that in fact they can be seen in a humanities context to constitute our world in temporal relation. The primary tool for generating such contradictory diagrams of human experience is described below.

\section{Nowsliding: an Inner Standing Point}

Our policies of anticipating (and welcoming) the upload of user-designed graphic notation, of enabling easy transition among multiple modes of diagrammatic and conceptual reasoning, and of enforcing the formalization of interpretive models in the loosest terms possible are examples of Temporal Modelling's encouragement of hermeneutic practice in computational contexts. In some sense, this practice - 
exemplified by users' saving and exporting of visually-constructed models as XML structures - is still external to the composition environment we have built, even as it forms an integral part of the methodology Temporal Modelling is designed to reinforce. I will close this discussion by describing what we consider our most exciting tool for encoding interpretation and subjectivity within the designed Temporal Modelling environment: the mechanism we call the nowslider.

Nowsliding is a neologism for an interpretive exercise all of us undertake constantly on which, to some degree, our understanding of ourselves and of our lived experience depends. Likewise, it is an exercise intimately familiar to students of imaginative literature and other content areas in which subjective interpretation of time and temporal relations is a shaping factor. ${ }^{33}$ The Temporal Modelling Project defines nowsliding as the subjective positioning of the self (or of any interpreting agent such as a fictional or historical personage or perspective) along a temporal axis and in relation to the points, events, intervals, and inflections through which experience is classified and time made meaningful. You nowslide when you picture your world at the present moment and, some ticks of the clock later, again at another ever-evolving present. You nowslide, too, when you imagine and project the future or interpret and recall the past. Our toolset allows a graphic literalization of this subjective positioning and temporal imagining, in the shape of configurable, evolving timelines whose content and form at any given "moment" are dependent on the position of a sliding icon, representative of the subjective viewpoint. 
In other words, we have literalized a notion of temporal subjectivity as a Rossettian "inner standing point" (serving as a "locus of interpretation within" historical or narrative invention) into a concrete and adaptable control mechanism in the PlaySpace (cf. Drucker and Nowviskie, Appendix A: "Special Interface Features"). Modelling of multiple independent or interdependent points of view is possible in the context of a single set of data, and the visual characteristics through which a nowsliding event is expressed may be specified by a user in the construction of a particular model. In this way several interpretive perspectives can be modelled and diagrammed even on a single axis, which facilitates their interrelation in novel ways.

At present, two display modes for the nowslider are in development. ${ }^{34}$ The first is a catastrophic mode, in which new axial iterations (or imagined past- and future-lines) spring in a tree structure from well-defined instances on a primary temporal axis. In this way, PlaySpace users can express the human tendency to re-evalute the past or make predictions about the future in the face of sudden, perspective-altering events. New subjective positions on the primary axis of time (and new happenings, figured as “initiating instants") can provoke more iterations, which do not supplant past imaginings or interpretations, but rather co-exist with them, attached as they are to a different temporal locus - the moment that spawned a new imagining. In this way, timelines are made to bristle with possibility, while still preserving a distinct chronology and single fundamental path in the form of the primary axis, against which 
all other measurements are taken (Figure 4). More explicitly topological

expressions of the notion of "catastrophe" in terms of a nowslider mechanism are planned for future implementation.

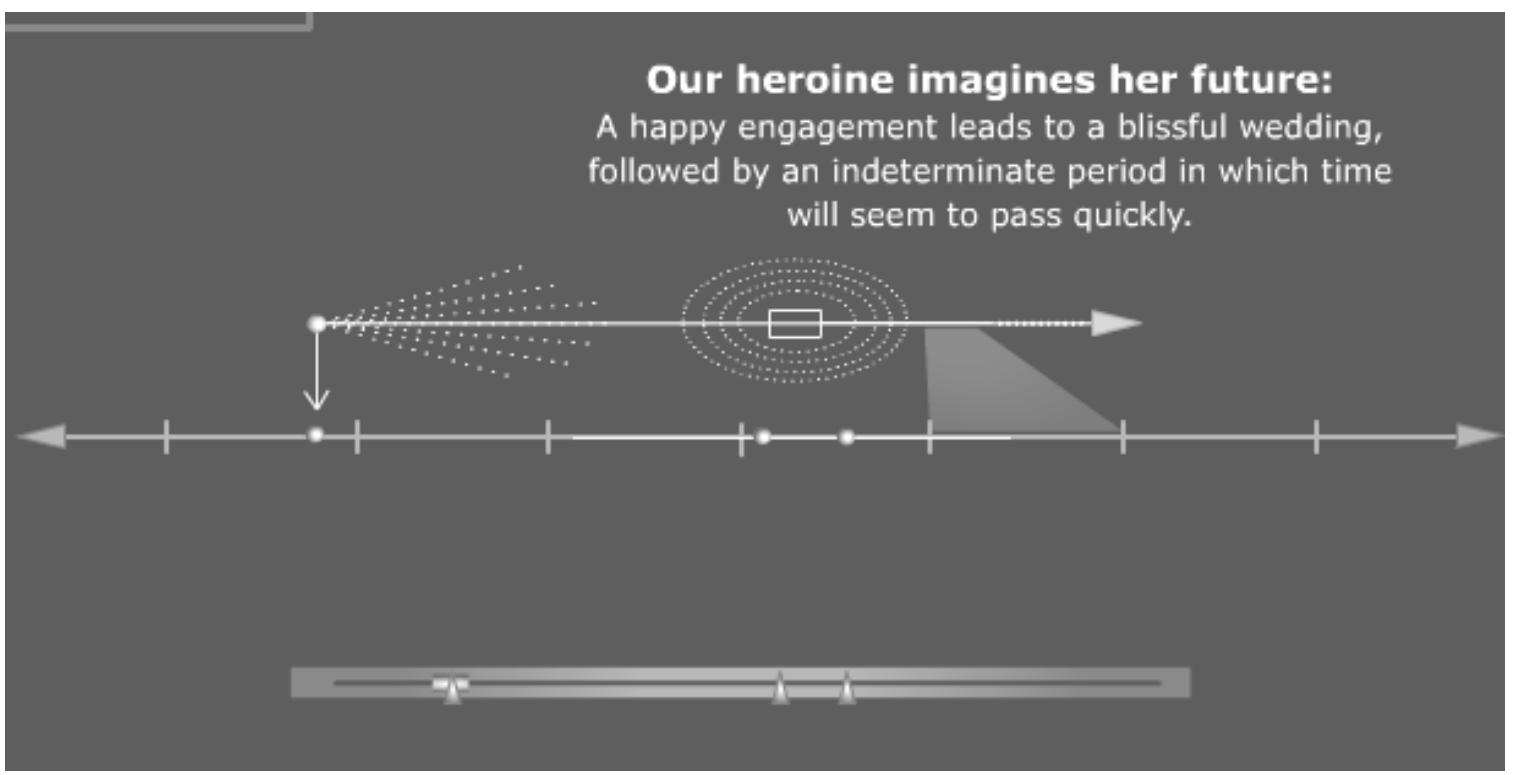

4a. First Stage: forward prospect

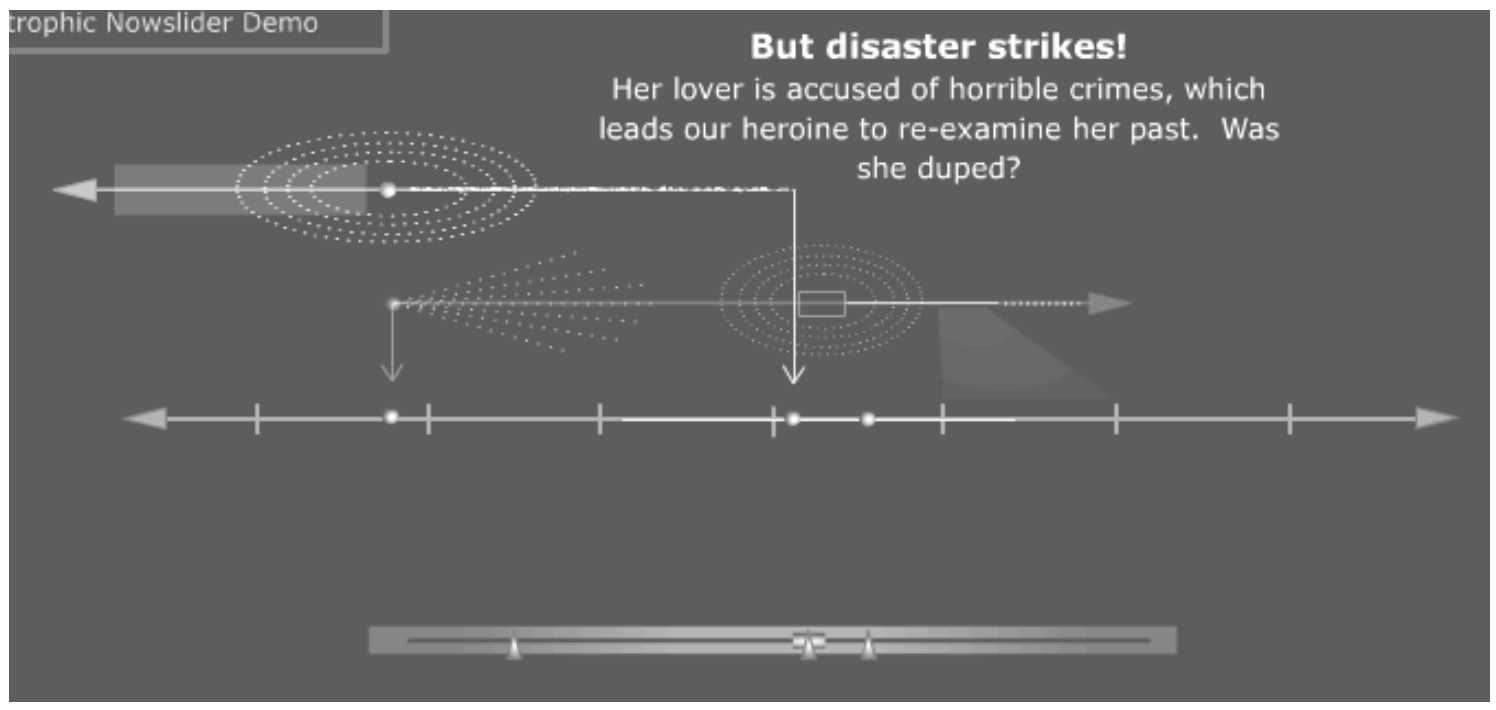

4b: Second Stage: backward retrospect 


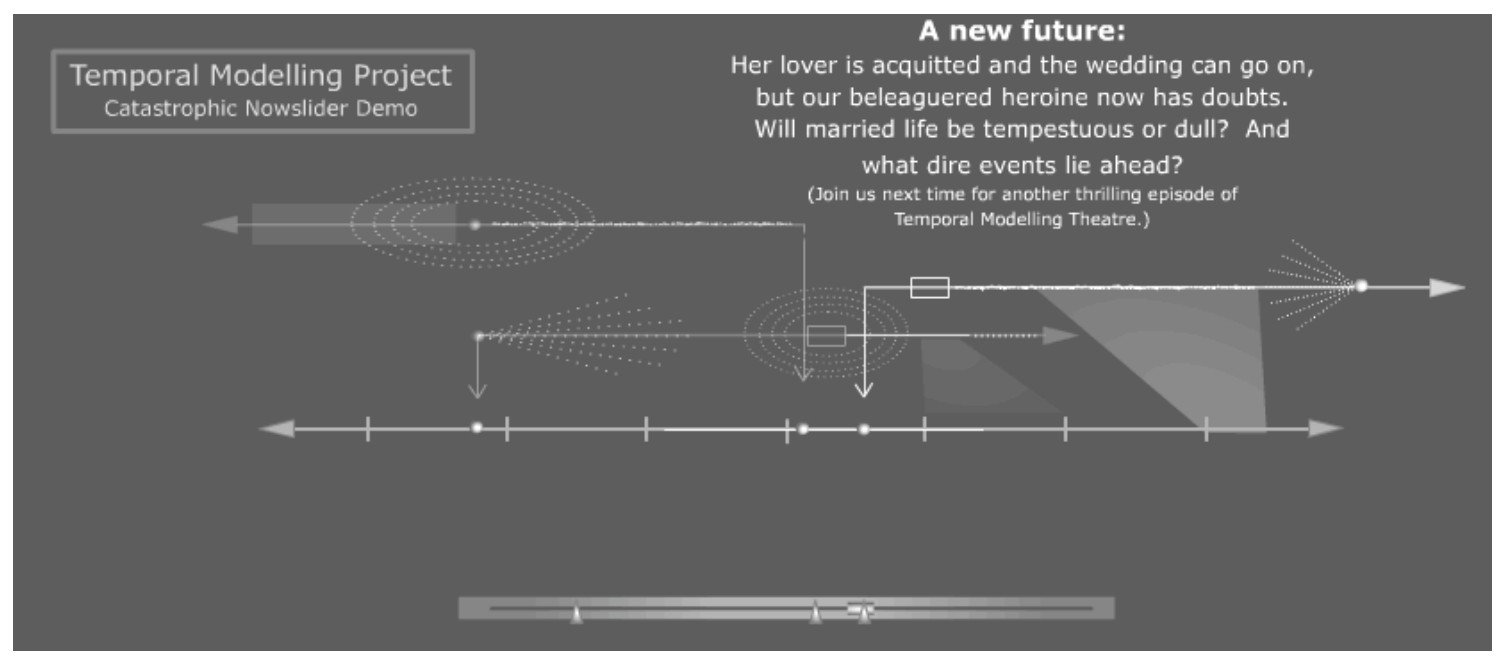

4c: Third Stage: revised forward prospect

[ Figures 4abc: Catastrophic Nowslider Screenshots and Descriptions ]

Our nowsliders also function in a continuous mode - distinct from the catastrophisms of intiating instants and of collapsing basins of order — in which past and future iterations fade in and out, change in position or quality, appear or disappear, all within the primary axis of the subjective viewpoint. That is to say, forward motion "in time" of an icon representing a particular, subjective viewpoint causes the timeline itself, and all the other objects that, at any given moment, might appear fixed and external, to shift and mutate. No new lines are spawned; instead, this mode presents time as a continuum of interpretation, in which past and present are in constant flux and their shape and very content are dependent on the interpretive pressure of the now (Figure 5). 
Temporal Modelling Project

Continuous Nowslider Demo

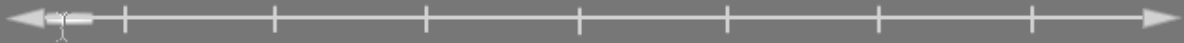

Temporal Modelling Project

Continuous Nowslider Demo

Engagement! A joyous mood, an extended (imagined) future together...

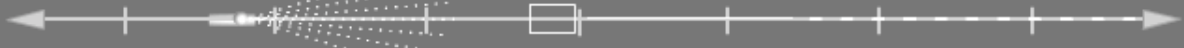

Temporal Modelling Project

Continuous Nowslider Demo

and the wedding date gets established.

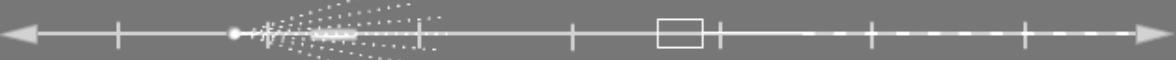


Temporal Modelling Project

Continuous Nowslider Demo

and the wedding date gets established.

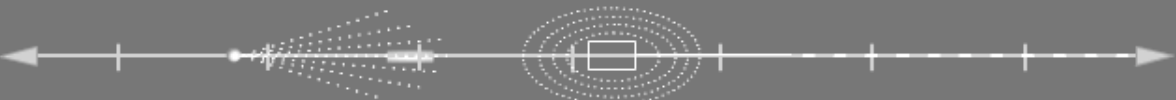

Temporal Modelling Project

Continuous Nowslider Demo

Alas! The bridegroom goes to jail!

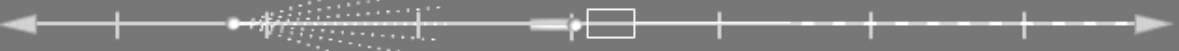

Temporal Modelling Project

Continuous Nowslider Demo

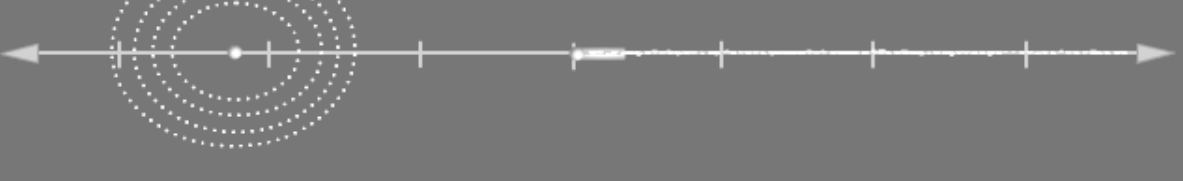


Temporal Modelling Project

Continuous Nowslider Demo

...which changes everything. Why did she say yes? And what will the future hold?

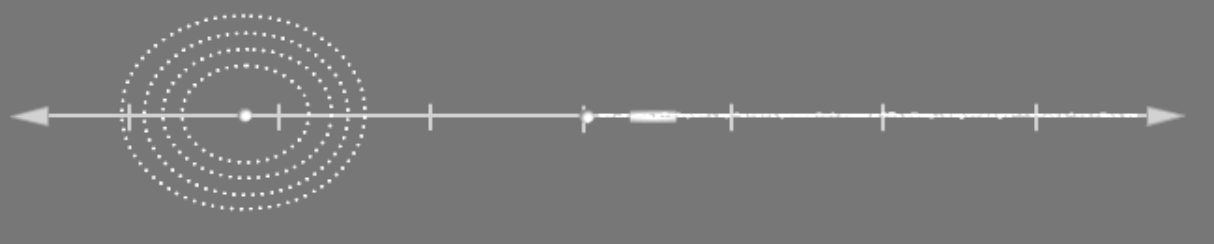

Temporal Modelling Project

Continuous Nowslider Demo

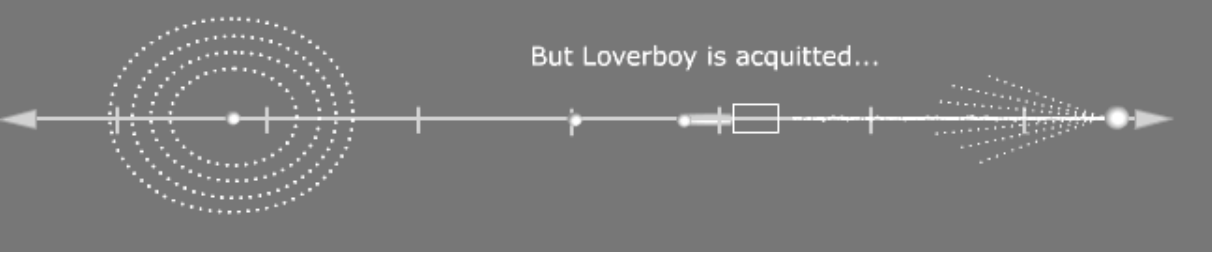

Temporal Modelling Project

Continuous Nowslider Demo

And, despite her misgivings, the wedding will go on.

A pall falls over their past together, and she dreads an unknowable future disaster.

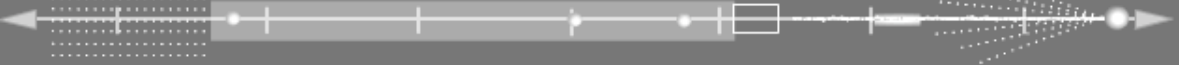


Temporal Modelling Project

Continuous Nowslider Demo

And, despite her misgivings, the wedding will go on. A pall falls over their past together, and she dreads an unknowable future disaster.

Temporal Modelling Project

Continuous Nowslider Demo

Even as her terror fades, the shadow of the past persists.

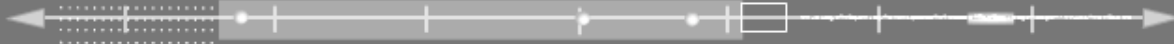

Temporal Modelling Project

Continuous Nowslider Demo

(The course of true love never did run smooth.)

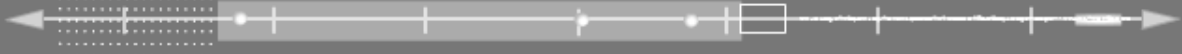

[ Figure 5: Continuous Nowslider (a cut-your-own flip book)] 
Continuous nowsliding is highly dependent on the motion of the controllermechanism as it can be manipulated by the model's creator during editing or by a viewer in a display format. A static screenshot does this brand of nowslider little justice. But more: the patterns of use it requires make a theoretical point about temporal subjectivity and its visual expression. This mode (its very availability and its basic, diachronic display requirement) implies that, for some interpretive purposes, a static "snapshot" view of subjective positioning and iteration is necessarily impoverished. The digital medium permits a flexibility and fluidity in design that carries over into the sorts of analytical statements it facilitates. Just as easily — and inevitably — certain interpretive acts vis-à-vis subjective positioning will be thwarted by our interface. We can only hope that the relative richness and user-customizability of the PlaySpace offsets its limitations, and that the visual and analytic constraints it places on users will (like all constructive systems of constraint) spur them on to resistant acts of creativity.

\section{E. Directions: A Cognitive Science Approach}

We have seen that the analysis and visual representation of temporal relations as part of lived experience is a practice with a long tradition and established methodology, both in scientific and interpretive disciplines. However, our Temporal Modelling Project takes shape at an historical moment in which newly-facilitated computational work on time focuses much attention on concepts suddenly (fruitfully) in flux. Our own intense pragmatic concentration on modelling time and temporal relations as they are 
constructed by subjective interpreters is part of a larger growing interest in cognitive science fields in dynamics, especially the dynamics of internal mental representation - how we sentient beings represent ourselves in time, to ourselves (Port \& van Gelder; Bickhard; Christensen \& Hooker).

The temporal dimension of such representation has, after a long period in which static cognitive models predominated, become central — to such a degree that recent research outlines the functional profile of a mental state in terms of that primary aspect of temporalized interpretation: causality. A mental state becomes, in fact, "the set of all causal paths running through it” (Bickle 198). Causal paths may be mapped as they play out over time, and Catholijn Jonker, Jan Treur, and Wouter Wijngaards' development of a "temporal modelling environment for internally grounded beliefs, desires, and intentions" seeks to do just that (Jonker et al 2003).

Construction of this computational environment is predicated on the BDI (belief, desire, intention) model of thought and action common to cognitive science approaches. The crucial difference is that Jonker adds a temporal dimension to an otherwise static model, treating all three elements as states open to change, and which take time to play out. Jonker employs a notion of "real time" rather than relying on instantaneous computational steps in order to produce a "more sincere formulation" of a subject's mental state than that commonly available to BDI applications (5). In other words, as in our own temporal schema, delays between causes and effects and the durations of 
cause and effect situations are appreciated. Jonker's model, unlike many BDI approaches, also takes mind-matter relationships into account just as we permit our users to do with the interrelation of objects and inflections in the PlaySpace (24).

This cognitive science work in defining a temporal modelling language for mental states is related to Leyton's "process grammar" for shapes (reviewed above in relation to syntactic inflection) in that its ends are predictive rather than reflective. Jonker and her colleagues mean to enable simulation in a software environment (written in $\mathrm{C}++$ ) to "automatically generate the consequences of... temporal relationships" (10). This is done "by generating consequences over time from the specified set of temporal relationships [in her modelling language], according to the paradigm of executable temporal logic" expressed by Barringer (Jonker et al, 2; see Barringer et al). In Jonker's digital environment, a set of temporally-related beliefs, desires, and intentions is attributed to an agent, and an algorithmic "simulation determines the consequences of the temporal relationships forwards in time" (10).

Jonker's taking into account of the passage of time is an advance in cognitive modelling; however, her definition of "real time" does reinforce the common rationalistic temporal assumptions against which our own project positions itself: time is figured in her model as linear, neutral, and homogenous. And, of course, the automatic and immutable generation of "consequence" from an invariable set of externally-applied interpretive rules flies counter to the concept of the ludic algorithm expressed in the Temporal 
Modelling PlaySpace. The diagrams and equations that result from Jonker's simulations participate in Unsworth's paradigm of aesthetic provocation (discussed above, I.B2), in that they use algorithmic processes to tease out for viewers a new and valuable picture of information already fundamentally embedded in the structure at hand. In contrast, our own model of temporal relations as they connect to subjective belief, desire, and intent is designed to support a constructive environment where users can express even — or perhaps especially — illogical consequences playing out over time.

Another crucial disparity in these two "temporal modelling" systems lies in their different approaches to the possible co-existence of conflicting belief systems. The Temporal Modelling Project enables the graphical representation not only of conflicting beliefs, but of alternate and co-existing realities in the form of multiple, equally-weighted temporal axes in catastrophic and constantly-morphing primary axes in continuous nowsliders. In contrast, Jonker's model enforces a simple relationship between event and interpretation along a single, authorized and regular temporal axis: When the world situation suddenly changes, the beliefs [of a subject $]$ may follow suit. The belief B1 and the belief B2 of two opposite world properties should not hold at the same time; they should be exclusive. As the external world state fluctuates, the beliefs should change accordingly, but never should there be both a belief for a world property and a belief for the opposite world property at the same time. (8) 
Our nowslider mechanism is meant to enable and encourage, for the purposes of humanities interpretation, exactly the opposite sort of thinking Jonker's software specification enforces. In our view, human interpretive systems and the documentary records on which they act and through which they are expressed embody (and in fact depend upon) the possibility of conflicting yet congruent beliefs.

The most valuable aspect of Jonker's work to a humanities computing project such as ours is not the resulting main piece of software - which creates, for example, graphs of a mouse's belief, desire, and intention to eat cheese charted against its cycles of hunger and the presence or absence of a barrier that thwarts it — but rather her "intentional attribution checker," a subroutine which works backwards from observed or modelled data to flag seemingly spontaneous actions not explainable by a temporalized intentional relationship and to mark instances where a predicted action, for reasons unaccountable to the model, did not occur. The output of this procedure appears as an equation written in Jonker's temporal BDI language, translating roughly to "true" or "false."

However: "in addition to checking whether the rules hold, the checker produces an informal reading of the trace. The reading is automatically generated, using a simple substitution, from the information in the intentional trace" (12). This customizable "informal reading" articulates the beliefs, desires and intentions of the subject relative to 
its environment (for example, "the mouse desires and intends to go to point $\mathrm{P}$ and believes that no obstacle is present") and the behavior model governing the checker (“absent perceived or real obstacles to an intended and desired goal, the mouse will proceed directly toward the goal”). It likewise expresses violations of the intentional model in natural language (which could be made as "natural" as its user desires): "Despite its hunger, the mouse unexpectedly takes the scenic route to the cheese at point $\mathrm{P}$ via point $\mathrm{B}$, thereby beginning to violate the given rules of the model. It eventually reaches $\mathrm{P}$ as expected, stopping the rule violation.” (17).

It is easy enough to imagine this intentional attribution checker being applied by humanities scholars to models developed in a narrative or historiographic context. Its inviolate inflexibility and mechanistic output are - perhaps paradoxically to those whose stock and trade traditionally lie in analogic thinking and (at least) seven types of ambiguity - its greatest strengths. At what point might Hamlet's actions violate the interpretive ruleset (the hypothesis or model) set up for him by an algorithmic translation of Greenblatt's critical stance? Of Bloom's?

Jonker et al. provide an example of narratological application in an analysis of a children's story — for her purposes, in order to determine the intentional understanding of three- to four-year-old children (20-22). Here, inconsistencies are flagged either when the agent (fictional Jason, a little boy who would rather play in the snow than go to football practice) doesn't do as the model expects or when the 
environment (the bus meant to take Jason to the football field) doesn't do as the agent expects. Happily for Jason (and quite predictably for any reader familiar with the narrative conventions of childrens' literature) the bus-driver gets lost and winds up stopping in the snowy mountains rather than on the playing field. The simplistic, mechanical representation of intention played out over time in Jonker's software may look a bit tedious at first, but the defamiliarizing effect of watching those narrative events that just seem "right" marked as logical inconsistencies soon becomes thrilling.

Imagine a scholar inputting a model of cause and effect which seeks to explain patterns of accusation, conviction, and acquittal during the Salem Witch Trials — the precise interest of a notable IATH project currently in partnership with Temporal Modelling. If we could apply this sort of algorithmic analysis to a model created in the PlaySpace, the scholar's attention would be drawn to loci where his interpretive premises don't hold true. (For example, the mechanism might analyze the encoded temporal and geographic parameters of information extracted from historical documents in order to flag violations of a scholar's algorithmically-specified hypothesis about the role of time and proximity in fostering witchcraft accusations. Inconsistent "accusation events" plotted on a graphical timeline could then be highlighted so that their own internal patterns become more comprehensible.) Such an application of Barringer’s “executable temporal logic" within an editing and model-making environment like the PlaySpace could first spur users to develop interpretive models that account fully for intentional inconsistencies and then perhaps start to draw other deeply-embedded, unconscious 
assumptions to their attention.

In other words, negative results could have positive consequences for the refinement of a model, in just another example of the "importance of failure" in humanities computing (Unsworth). The interesting thing about the application of executable logic within the PlaySpace is that it would make the temporal modelling process explicitly self-testing, in the same way that Llull's combinatorial wheels invite the adjustments of an artista based on logical analyses or intuitive assessments occuring exterior to the modelling system.

Does the very presence of a refinement process enabled by algorithmically-enforced temporal logic somehow assume that a "correct" interpretation is possible? Knee-jerk relativism is probably unhelpful here. Certainly the Salem Witch Trials project would like to offer as complete and valid a model of the events of that annus horribilis as possible; on the other hand, the hallmark of good humanities scholarship lies in its proclivity to hold open the door for alternate creative interpretations, new models. This problem centers in the notion that true and false (binary values necessary to the functioning of Jonker's program) are not nuanced enough for most humanities purposes. The ideal situation would be one in which the Temporal Modelling PlaySpace could support integration of some executable logics but fundamentally remain a rich and evocative palette for the painting of subjectivities and interpretive expressions that don't necessarily lend themselves to mechanical verification. 


\section{F. The PlaySpace in Application}

The following four screen shots demonstrate the basic interface of the Temporal Modelling PlaySpace and show the tool as it might be applied to experimentation with an interpretive research problem. Here, the dataset takes an imaginary look at a real humanities computing project, the Salem Witch Trials archive (IATH; Benjamin Ray). We hope to begin real beta testing with the Salem project and other user groups in the summer of 2004, when important, in-process features such as the nowslider and inflection software have been implemented more fully. (Those features are therefore not presented among the screenshots that follow.) Please bear in mind that, simultaneous with the user's graphic sketching of timelines in these screenshots, the Temporal Modelling machine is always formulating and revising a rigorous XML model of the temporal relations given visual expression. A less photogenic interface for editing that XML has already been incorporated into Temporal Modelling; it is, however, not pictured here.

In the first screenshot, the user has created a single timeline at the center of the PlaySpace stage. The line has been populated with four points, one event, and one interval. Each of these "temporal objects" (including the axis of the line itself) has been given a text label, and the whole structure has been placed on a single, transparent layer. This layer, called "accusations," may be activated with the white tab at the bottom of the screen. Immediately above the tab is a set of display controls, including a 
slider for transparency or alpha value, a nowslider controller (the human figure), paired sets of controls for granularity and optical zooms, a panning tool, for sliding the contents of an entire layer around the screen, and a snap-to tool, for optimizing the user's view of a selected layer that may have been zoomed or panned excessively.

The long, dark bar to the right of the display tools is a viewslider, a special mechanism for handling Temporal Modelling's important re-figuring of the space of a computer screen. Because our tool is designed to allow unlimited access to the conceptual real estate existing along the horizontal axis (that is, to the left and right of the visible space bounded by the screen), the viewslider is necessary both as an orientation and navigational aid. The thin grey line inside the viewslider marks the average, relative size and position of timelines in the user's selected layer. When the user zooms out from the selected layer, this line shrinks. When the user zooms in, it expands - even to the point of extension beyond the edges of the viewslider's black rectangle, which scales appropriately. In other words, depending on the degree of zoom desired, the viewslider can represent a small grey line in a large, dark field, or a small, dark porthole on a long, grey horizon. The overall PlaySpace view responds to the user's clicks and drags inside the viewslider in a fluid way.

Tools for loading, saving, and editing PlaySpace models are located at the top of the screen. Pull-down menus give access to temporal objects and inflections, to contextspecific "help" features, and to an "inspector" though which various types of labeling 
(including text and color-coding) can be applied.

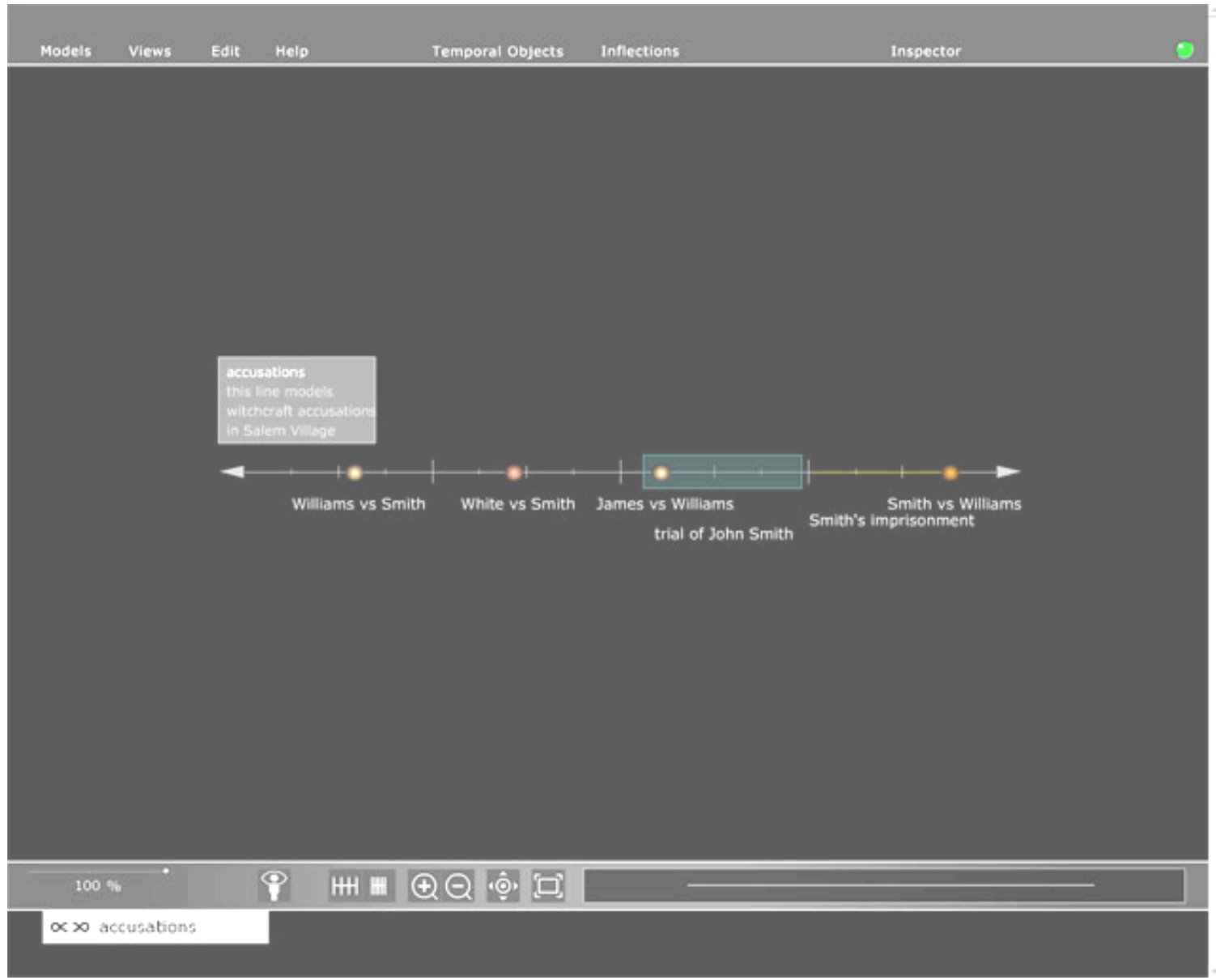

\section{[ Figure 6a: Temporal Modelling PlaySpace in Application ]}

The second figure shows the inspector, which is also designed to provide an alternate method for making those visual adjustments to models primarily effected with the mouse. Here, the user has opened an inspector tool for applying color-coding to a selected temporal object. Notice that the user can specify text labels for available color ranges, thereby creating a custom legend. The legend is model-specific, and can be 
saved and optionally re-loaded for future model-making. Color ranges (for example, the three tones from light to dark yellow visible at top) are built into the PlaySpace, but users can decide what they signify in the context of their saved models. In this example, the user has assigned degrees of "relative importance" to the three tones. Behind the color legend, a basic inspector for the "sermons" timeline is visible. Here, the user has assigned that axis a name and text description (visible on mouseover).

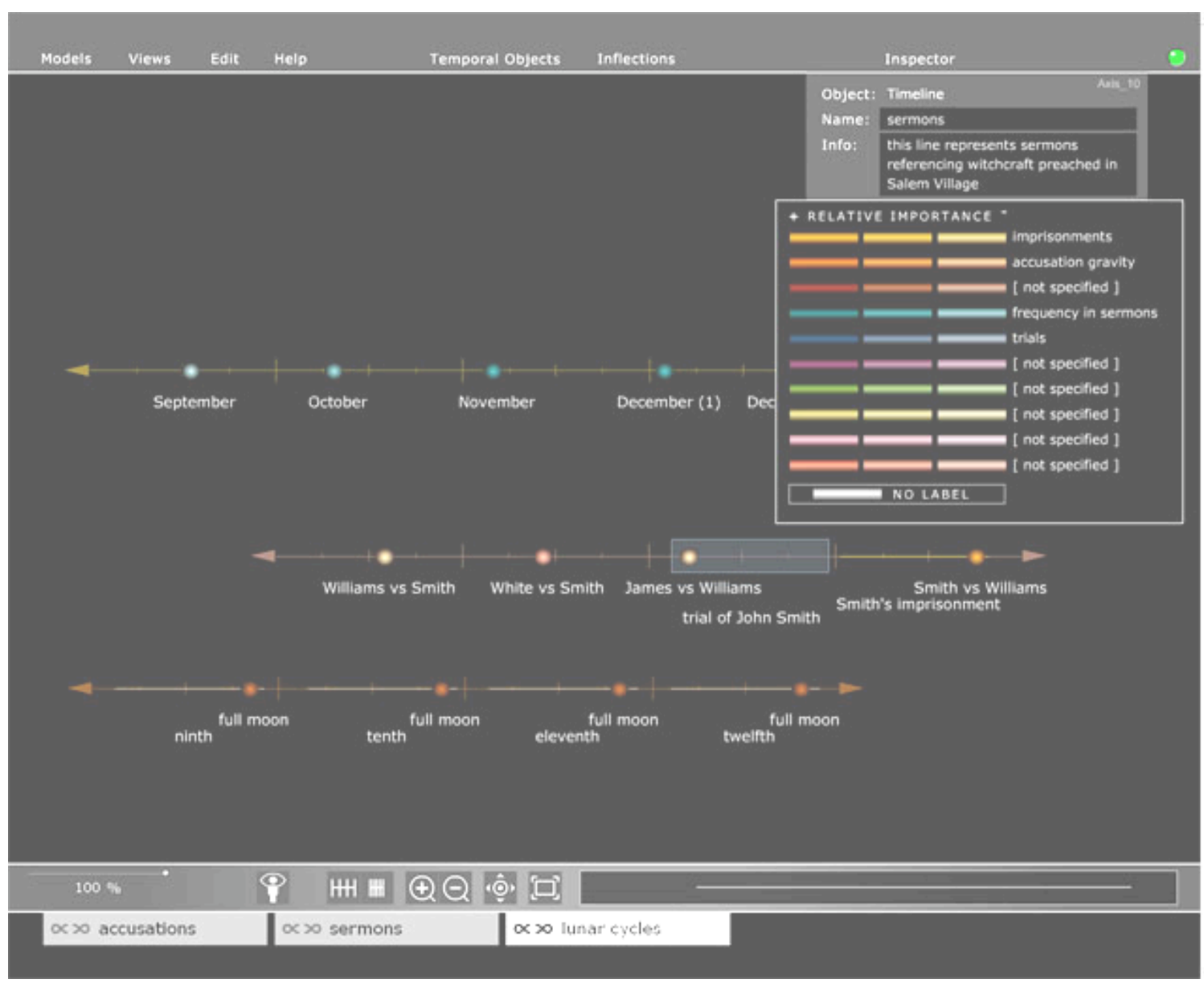

[ Figure 6b: Temporal Modelling PlaySpace in Application ] 
Three timelines, each on separate layers, have now been constructed in the PlaySpace. Our imaginary user is here charting imaginary patterns of witcheraft accusation against hypothetical lunar cycles and correlating that with the frequency (marked through color intensity) of appearance of certain Biblical passages in imaginary sermons delivered in Salem Village. In the third screenshot, two more layers and associated lines have been added. Now legislative cycles and pretend diary entries are in the mix. Notice that some layers have been zoomed in so as to show greater detail. This facility becomes particularly important when densely-populated timelines are the norm.

In our sample model, each timeline has been placed on a separate layer. This system is purely conventional and is a choice of the user. Depending on the data being modelled, other organizational schemes may be more appropriate. We have, however, built a tool for handling the visual characteristics (zoom, transparency, position, etc.) of layers both jointly and separately, in order to facilitate editing and comparison. Notice that, where in the previous image the layer tabs at the bottom of the screen showed "broken-link" icons, all layers in Figure 6c display fused links. The user has simply clicked on the link icons for those layers he wished to meld, thus joining them for panning, zooming, or other operations. This is purely a display feature, although the PlaySpace remembers these settings and applies them to the user's model the next time it is loaded. 


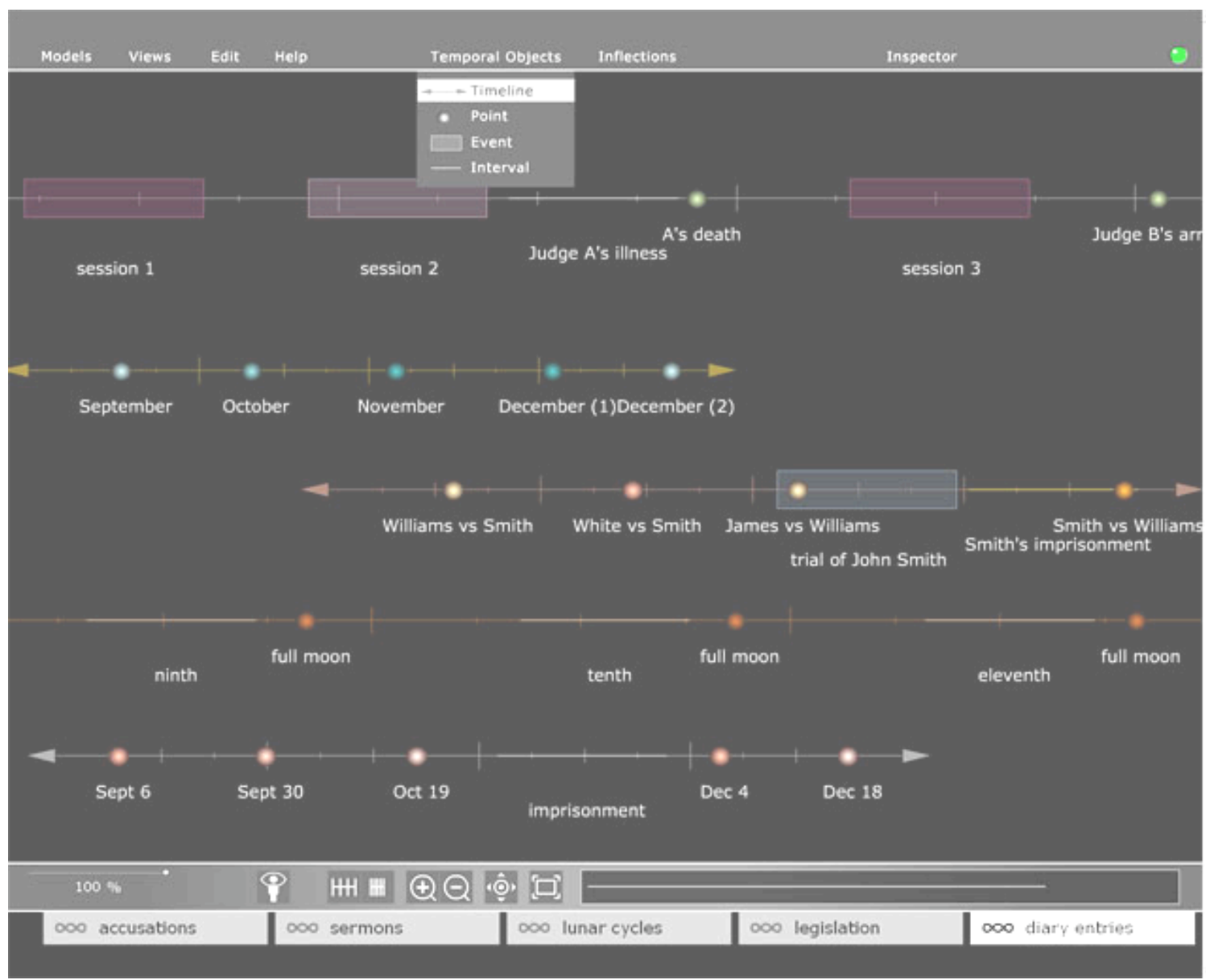

[ Figure 6c: Temporal Modelling PlaySpace in Application ]

The final screenshot shows an open menu from which the user can apply semantic and syntactic inflections to objects in the PlaySpace. This is done with click, drag, and snapto behavior identical to that powering the positioning of simple temporal objects. (Some very recent changes to this menu and the implementation of semantic "cancelled" and "special," and syntactic "causality" inflections by project programmer Jim Allman are not represented here, although their effects are visible in Imaginary Solution \#2:

Temporal Modelling at Sea.) Notice that our set of timelines and layers is zoomed out in 
Figure $6 \mathrm{~d}$ so as to take up only approximately fifty percent of the visible screen

space. The degree of zoom possible is actually much greater, and care has been taken to create PlaySpace graphics that are legible even at very small resolutions. This is simply another example of our project's commitment to the design of an open-ended modelling environment that makes as few assumptions as possible about the uses to which a rehabilitated "aesthetic provocation" should be put.

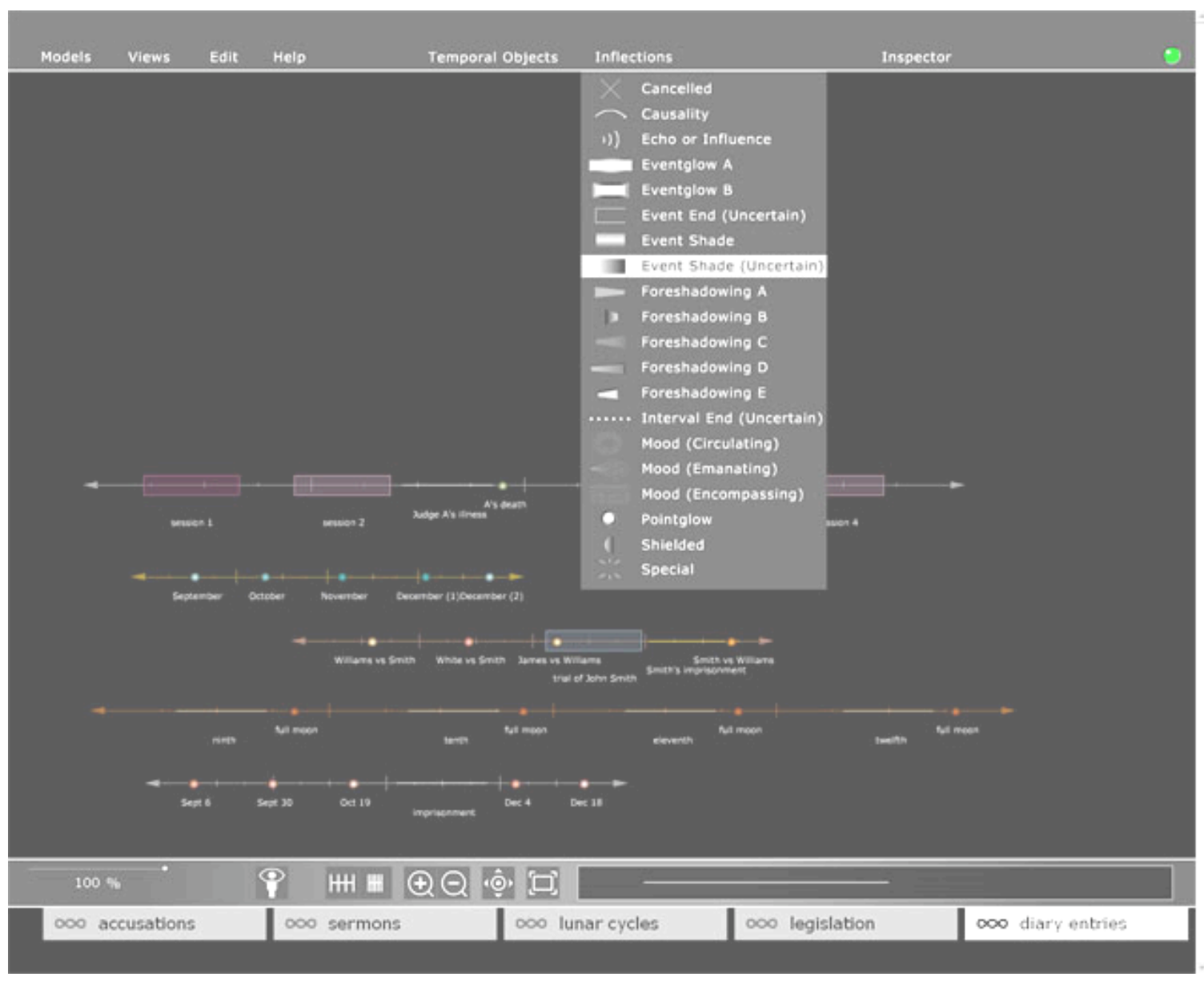

[ Figure 6d: Temporal Modelling PlaySpace in Application ] 
Our taking of temporal subjectivity and the shaping force of interpretation as the content and over-arching theme of our PlaySpace and DisplaySpace environments is meant to reinforce the goal of the Temporal Modelling tools, and by extension, the goals of SpecLab, the humanities computing research collective at the University of Virginia from which the projects outlined here stem. ${ }^{35}$ Our aim is to place the human interpreter inside a visual and algorithmic system, where his very presence alters an otherwise mechanistic process almost at the quantum level. Humanists are already skilled at the abstract classification and encoding that data modelling requires. We are beginning to understand algorithmic work and to appreciate the transformative and revelatory power of visual and structural deformance. We at least think we know what to do with a picture or a graph. What we haven't yet tried in a rigorous and systematic way is the injection of the subjective positioning any act of interpretation both requires and embodies into a computational, self-consciously visual environment. This is the contribution Temporal Modelling wishes to make to the methods and outcomes of digital humanities. 


\section{IMAGINARY SOLUTION \#2:}

\section{Temporal Modelling at Sea}

When you're thoroughly at sea, an imaginary solution promises no terra firma. Still, you might take one last bearing and somehow slip ashore. This, for instance:

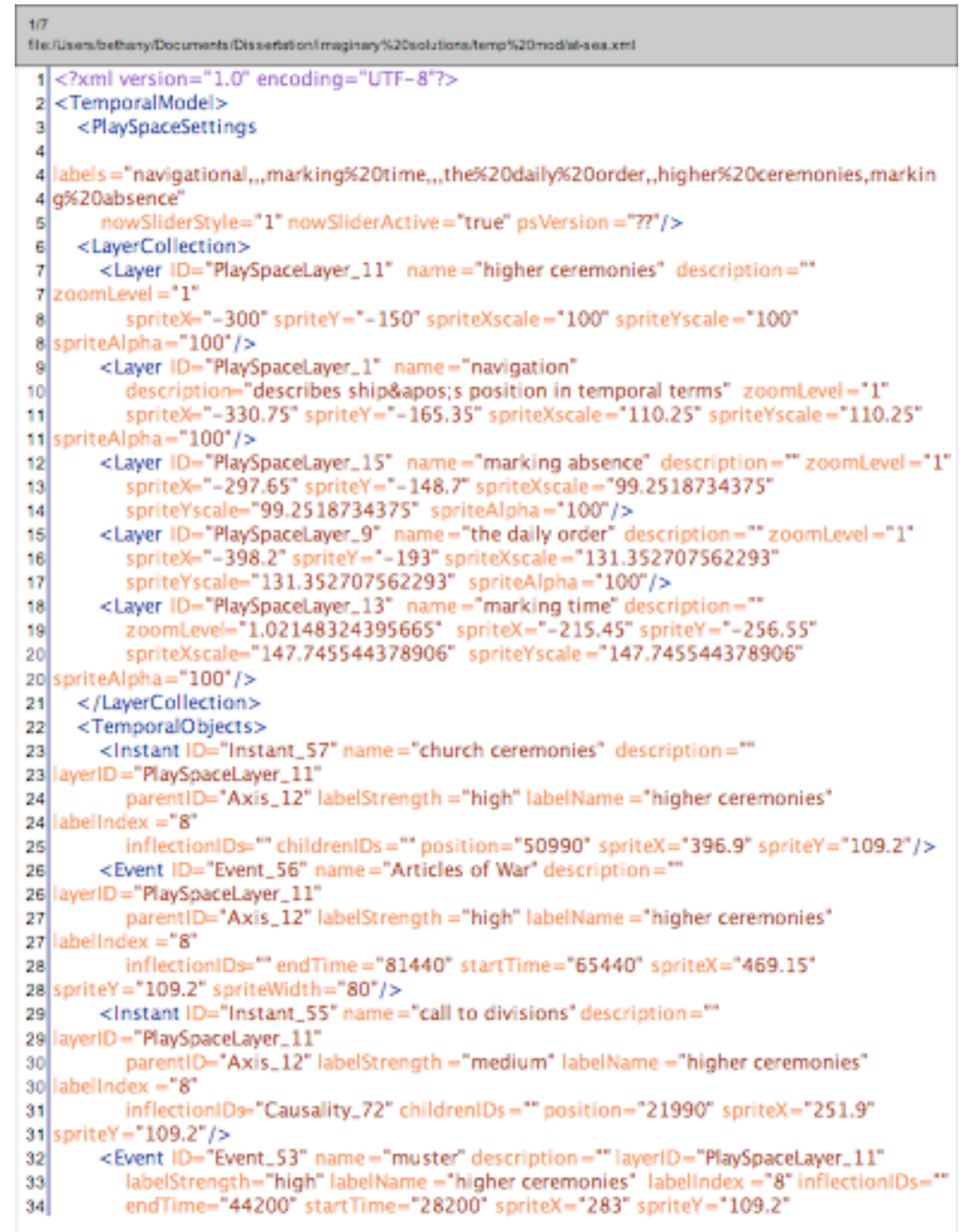




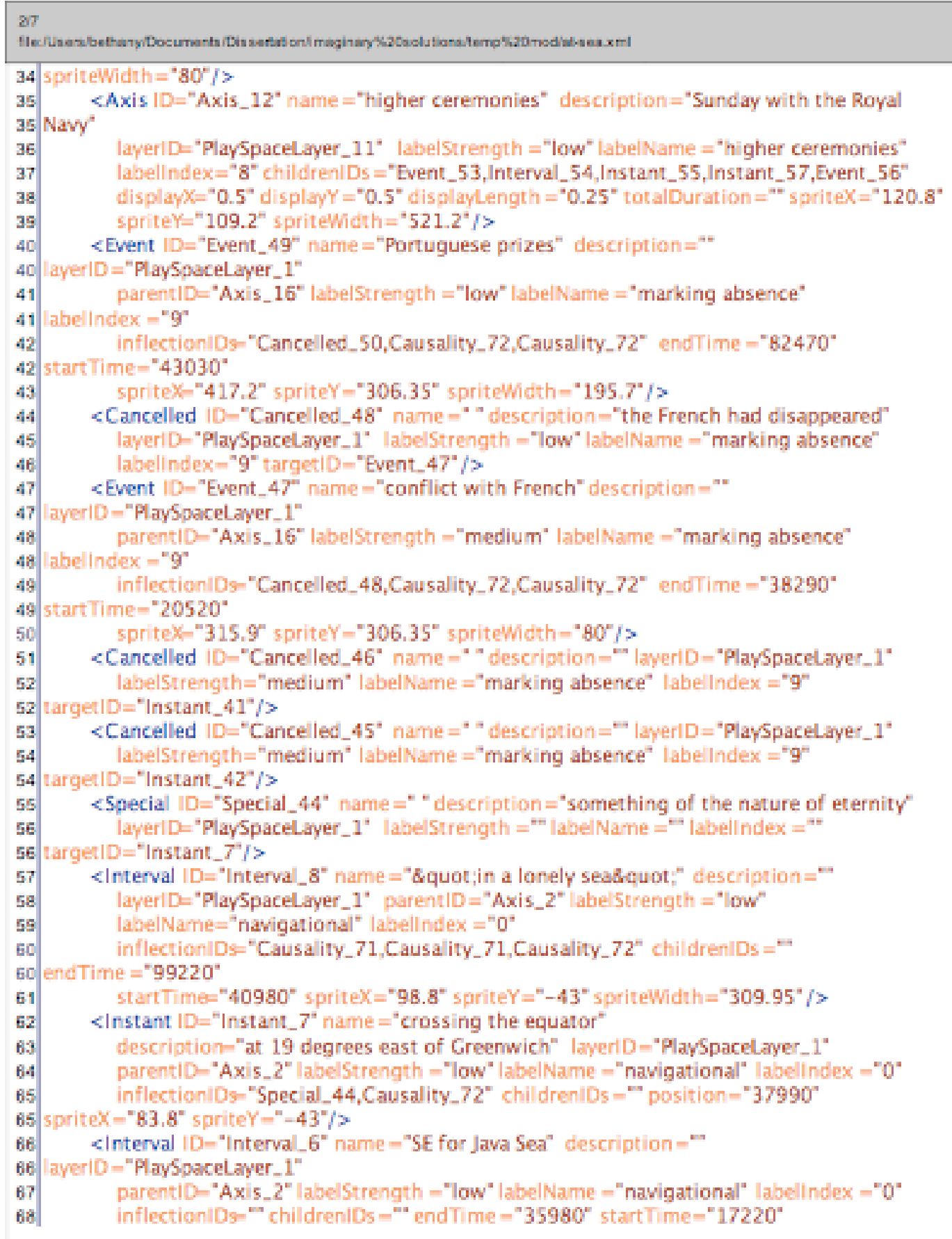




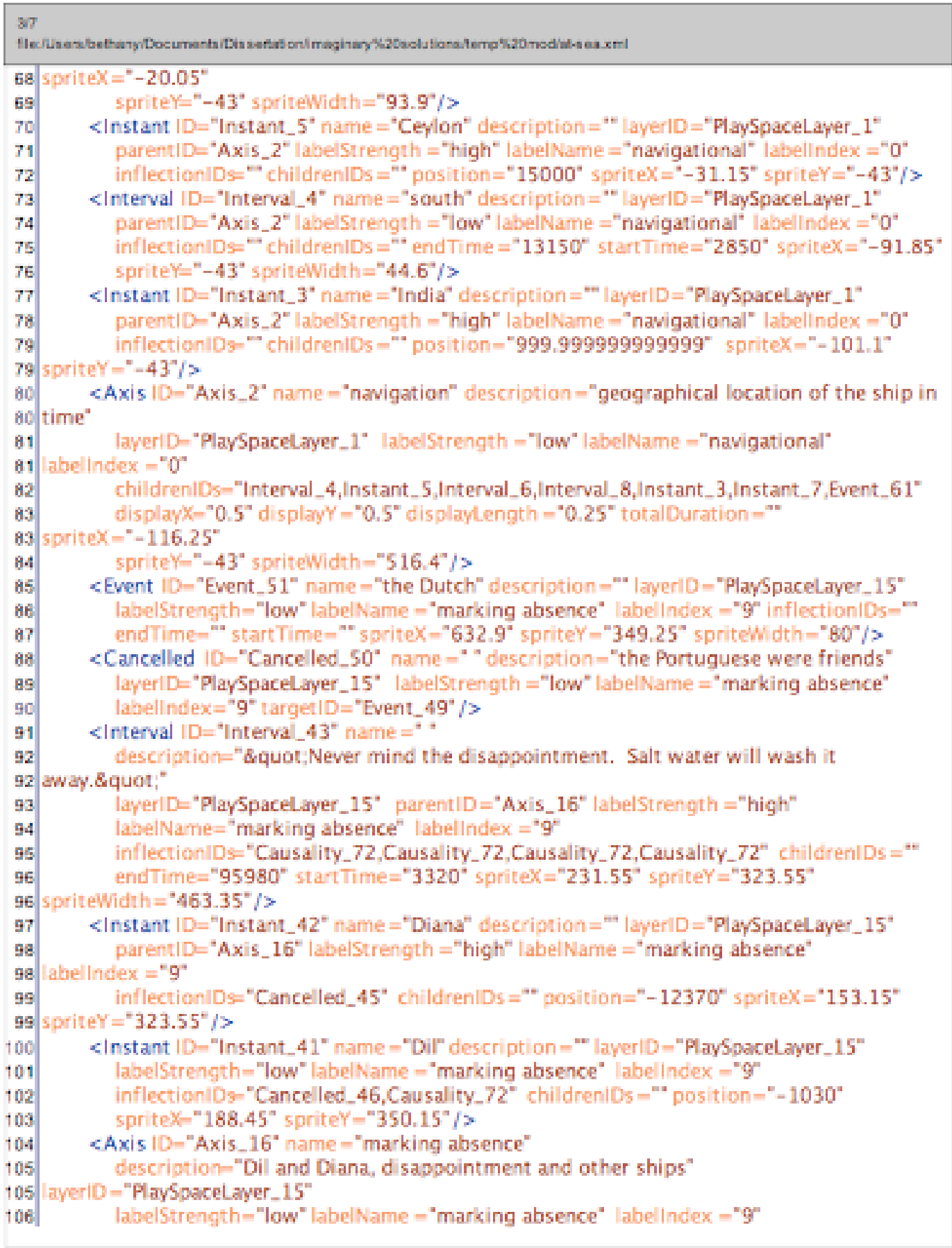




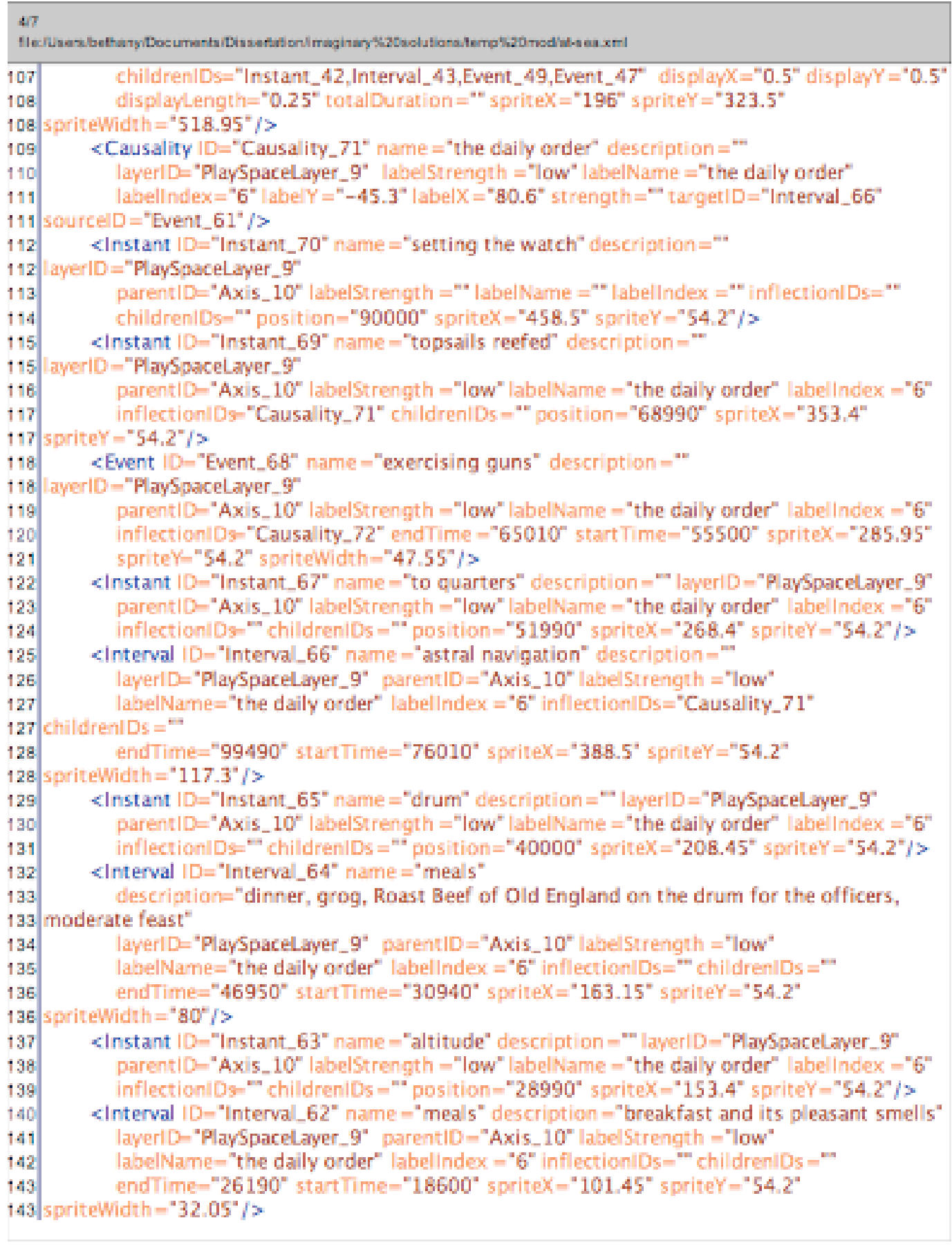




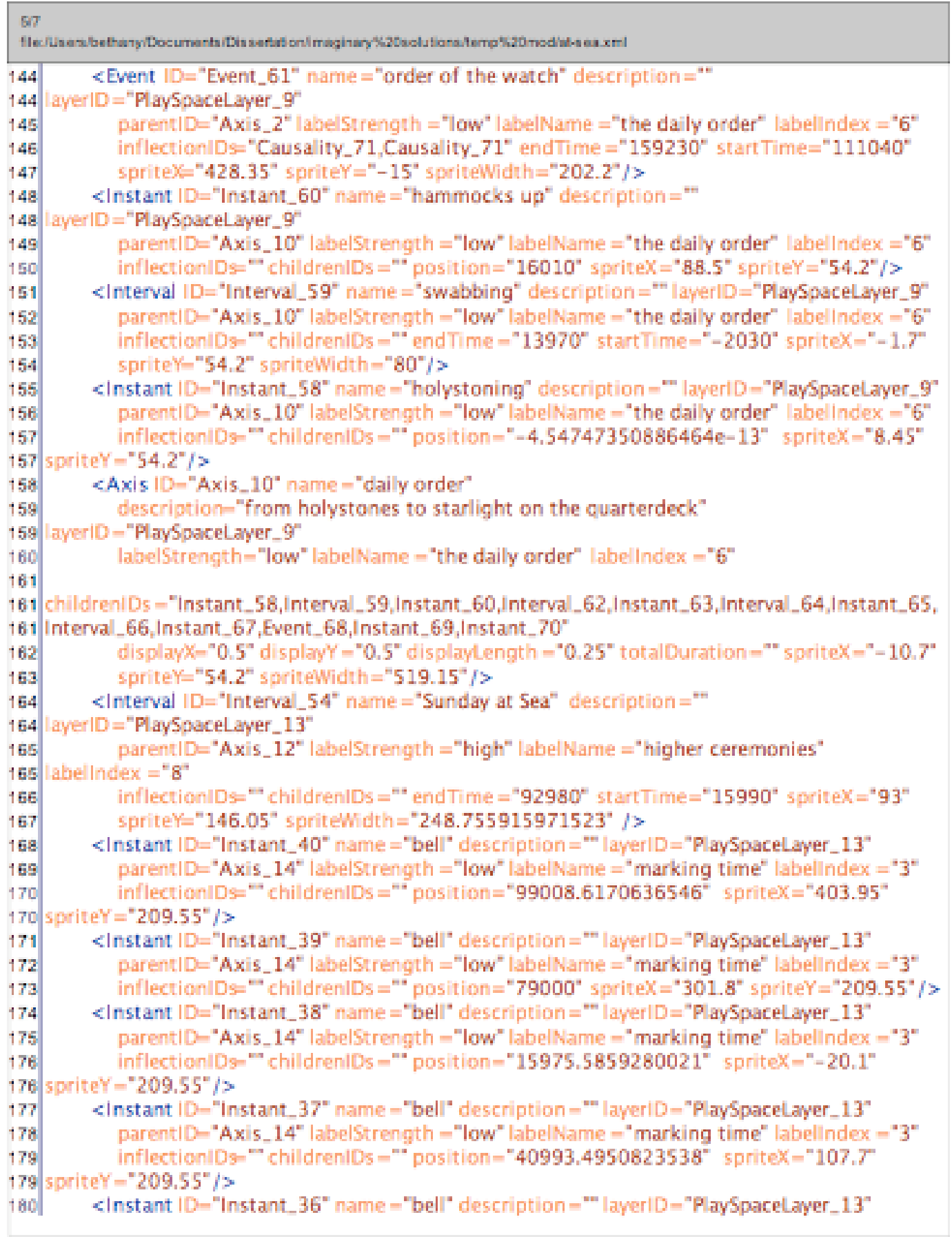




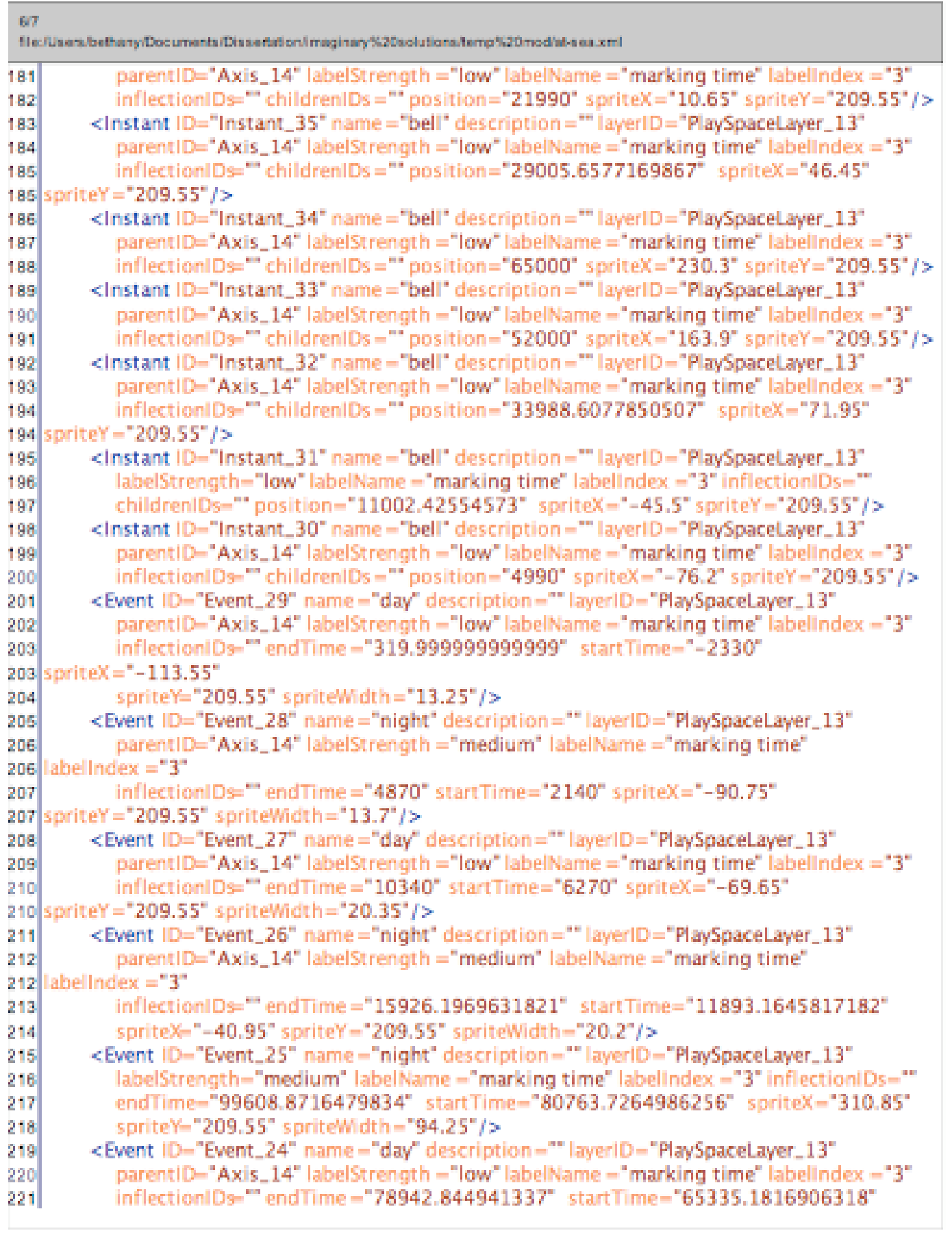




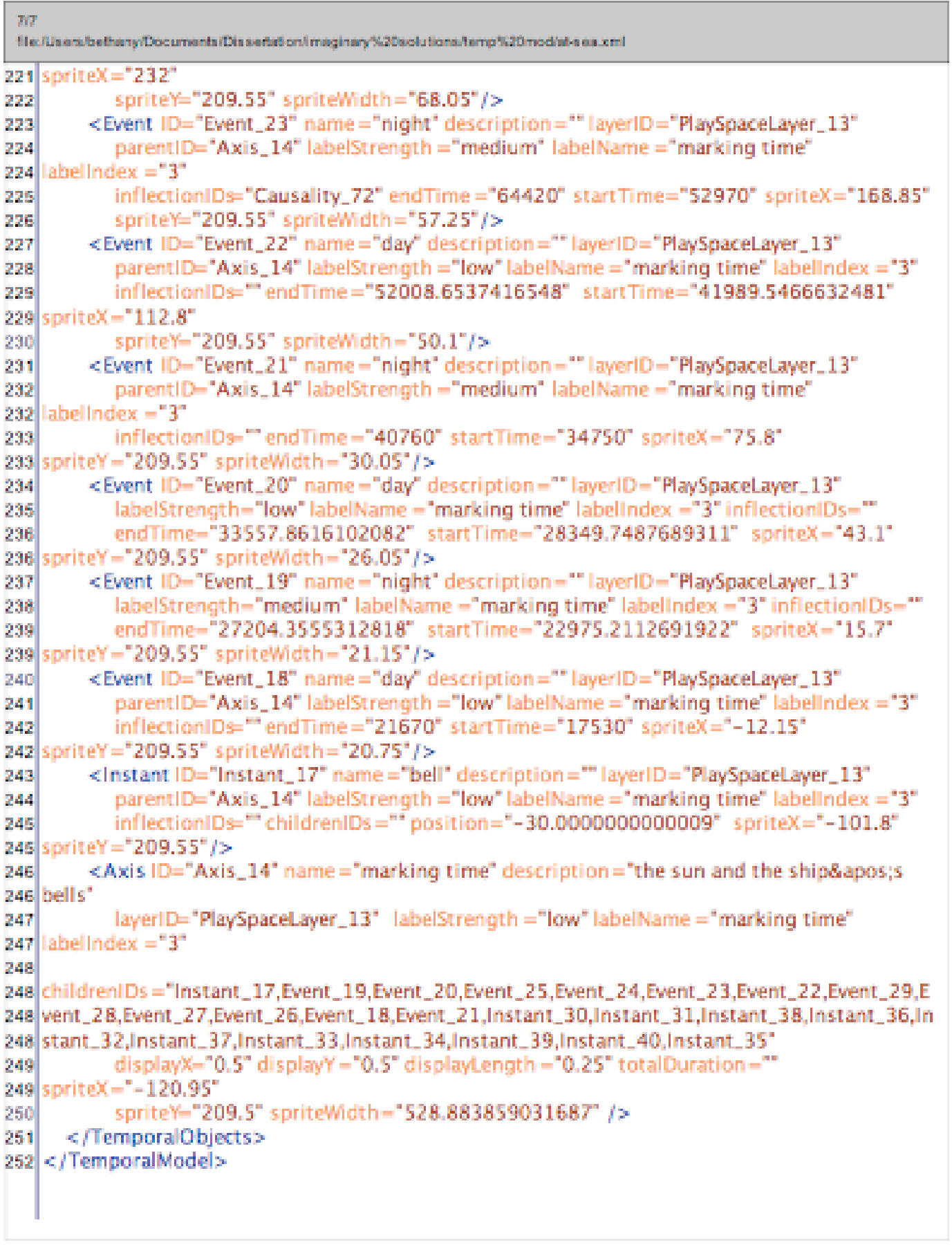


is almost (not quite) this:

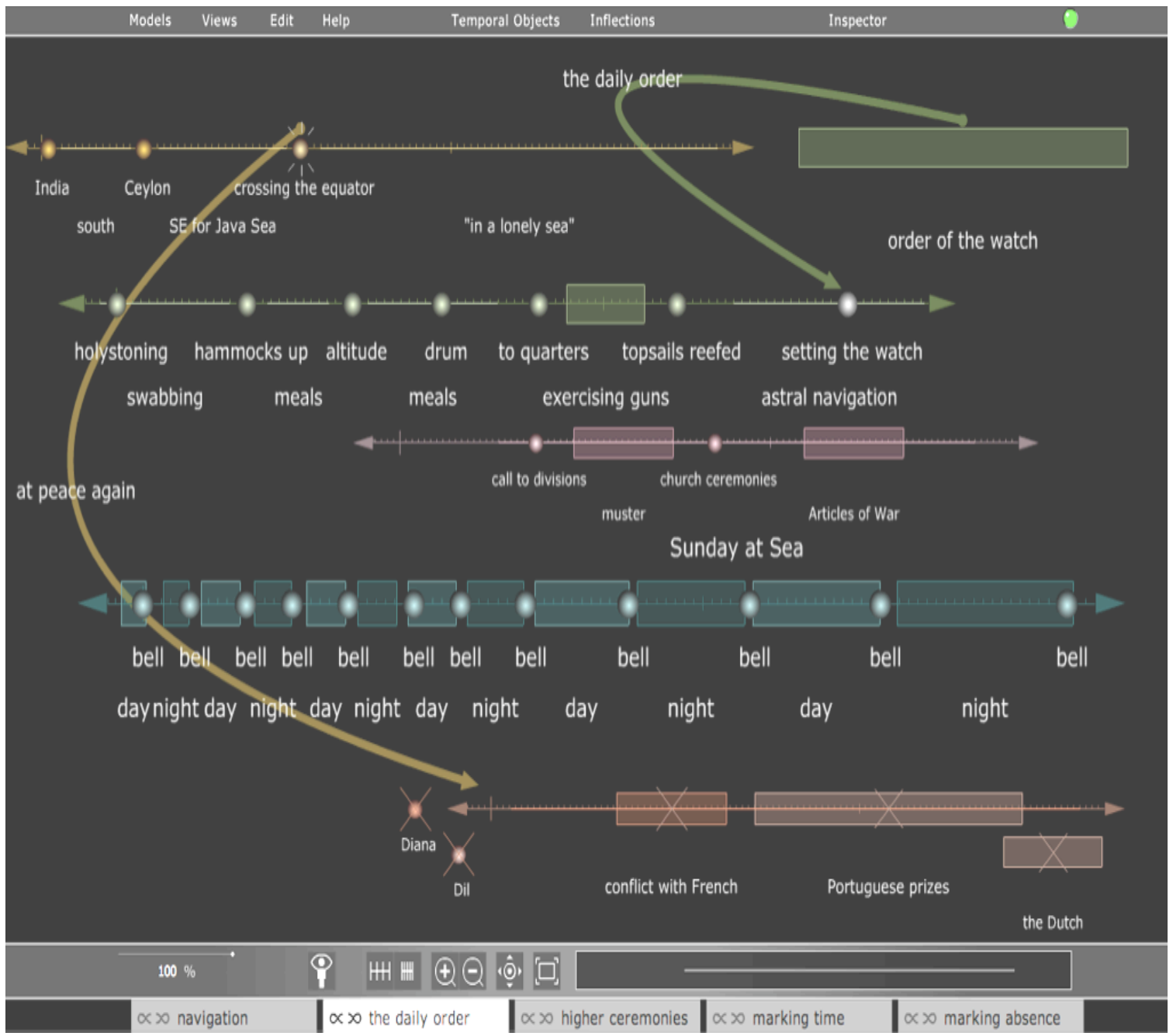

but it's not even close to this:

Never mind the disappointment. Salt water will wash it away. You will be amazed how unimportant it will seem in a week's time - how everything will fall into place. 
It was the true word: once the Surprise had turned south about Ceylon to head for the Java Sea, the daily order seized upon them all. The grind of holystones, the sound of swabs and water on the decks at first light; hammocks piped up, breakfast and its pleasant smells; the unvarying succession of the watches; noon and the altitude of the sun, dinner, grog; Roast Beef of Old England on the drum for the officers; moderate feast; quarters, the beating of the retreat, the evening roar of guns, topsails reefed, the setting of the watch; and then the long warm starlight, moonlit evenings, often spent on the quarterdeck, with Jack leading his two bright midshipmen through the intricate delights of astral navigation. This life, with its rigid pattern punctuated by the sharp imperative sound of bells, seemed to take on something of the nature of eternity as they slanted down towards the line, crossing it in ninety-one degrees of latitude east of Greenwich. The higher ceremonies of divisions, of mustering by the open list, church, the Articles of War, marked the due order of time rather than its passage; and before they had been repeated twice most of the frigate's people felt both past and future blur, dwindling almost into insignificance: an impression all the stronger since the Surprise was once more in a lonely sea, two thousand miles of dark blue water with never an island to break its perfect round: not the faintest smell of land even on the strongest breeze - the ship was a world self-contained, swimming between two perpetually-renewed horizons. Stronger still, because in these waters there was no eager impatience to see over the eastward rim: they sailed with no relation to an enemy, nor to any potential 
prize. The Dutch were bottled up; the French had disappeared; the Portuguese were friends.

- Patrick O’Brian. H.M.S. Surprise, New York: W.W. Norton \& Company, 1991.

Three points must be made.

\#1. The ocean's relation to temporal perception is a common theme in Patrick O'Brian's seafaring novels. Time pitches and bucks, dips and drags just like the surface of the earth against sea-stable legs whenever Lucky Jack Aubrey and his crew return to land. But (save in storm or battle) there's no irregular crush of events onboard ship. O’Brian's sailors already lie where they long to be.

The concept represented three ways above — as an XML data model, a PlaySpace visualization, and a text transcription — is, however, somewhat exceptional, because it spells out this relation (diffused through the 20 novels of the Aubrey/Maturin series) with great economy. It also marks a precise relationship between geography and temporality. Here, it's not just life "at sea" that inflects time, but rather life on the Equator at 91 degrees east of Greenwich. Aubrey's Surprise is scrupulously situated (by the "altitude of the sun" and through the "intricate delights of astral navigation"), but is nonetheless located in a far-flung, featureless space. Temporized geography swells to a "perfect round," experienced as "perpetually-renewed horizons" where not even the scent of a spot that marks time differently can be discerned. 
An imaginary solution to the problems posed by such a passage must recognize: geography is integral to the experience of time.

\#2. Once the "daily order seize[s] upon them all," two weeks' travel southeast of India brings O'Brian's sailors to a state in which the "higher ceremonies" of shipboard life mark the "due order of time rather than its passage." Gone is time's arrow, that "eager impatience to see over the eastward rim" that characterizes temporal representation in a variety of Western disciplines and against which our Temporal Modelling tool strains.

It doesn't strain hard enough. The current PlaySpace, which organizes and presents XML models against user-created "temporal axes," is not up to the task O'Brian's prose has set. An acceptable imaginary solution should understand that subjective agents can organize time linearly and cyclically — that the same stretch of time can be lived in both ways, and that one inhabited organizational system may quite easily bleed into another.

\#3. Stephen Maturin, the good ship's doctor to whom Aubrey addresses his advice ("Never mind the disappointment. Salt water will wash it away."), wants lavation. He leaves India having inadvertently caused the death of untouchable Dil, and with the fresh wound of Diana Villiers' rejection still untouched by time. The "disappointment" that fades away during his voyage to the Java Sea is therefore only one of many 
absences this passage of the novel marks. When shipboard life, still "punctuated by the imperative sound of bells... take[s] on the nature of eternity" and "past and future blur, dwindling almost into insignificance," other things fade out as well. The Surprise sails outside the common network of relations - personal, global, and temporal — that commonly define experience: "they sailed with no relation to an enemy, nor to any potential prize." The Dutch, the French, the Portuguese, are no longer on the map.

Any adequate imaginary solution would imagine absence, so simple to evoke in a book — so silently absent from digital representation in humanities computing. 


\section{Subjectivity in the Ivanhoe Game: Visual and Computational Strategies}

\section{A. Introduction to the Game}

The principles and practices of McGann and Drucker's IVANHOE project and its ludic expression in the Ivanhoe Game (as they have been fixed at given points in their evolution) are adequately documented elsewhere. ${ }^{36}$ This particular introduction, as befits my topic and the game itself - that structured subset of IVANHOE's larger interpretive and perspectival interests on which the following chapter builds - shows a more subjective slant.

I began the study that follows in order to make a practical contribution to ongoing work at developing a suitable Ivanhoe Game interface and corresponding architecture for the information (including aesthetic information) that players might generate in the course of gameplay. By the time I finished, I realized that I was really working to answer a personal question that emerged through several play-test sessions in which I participated — from the second instance of the game, played with the text of Wuthering Heights, to a Turn of the Screw play session meant to evaluate the impact of points and scoring on participants' actions and attitudes, to a little, closet-drama of a game, in which three players pushed quietly, steadily at the margins of Rossetti’s "Jenny." 37 
The tension I felt in these sessions (and in others) lay in my simultaneous desires - at once, to play a cooperative, social game in which an emergent interpretation of the work at hand could be shared, and at the same time to be left the hell alone, so that I could peacefully work through the private engagement I felt with my role and its texts. When my colleague Andrea Laue began experimenting with GraphVis software to generate visualizations of actual gameplay, I found physical evidence of that tension (Figure 1).

Here you see a diagrammatic representation of me - of my two roles and their moves in the game - as self-involved little islands to the far left and upper right, separated from the mainland of The Turn of the Screw and its community of players by a gulf that denied real, practical connection (in the form of explicit linking and commenting) at the same time that I felt that each of my moves embodied thematic and artistic connections to an overwhelming number of personally-defined textual "locations" in the discourse field of the game. That is to say, I knew my moves to be infused with subjective meaning and relation — in fact, with multiple subjectivities of their own, as well as of mine and of my roles — all of which remained uncapturable and (I feared) unable to be appreciated in the gamespace. So the question that faced me, appropriately, was a doubled one: How could IVANHOE, in the necessarily disambiguated software manifestation required by the Ivanhoe Game, not only allow but encourage the brand of subjectivity on which my playing style was predicated; and how could it do so while 
representing the simultaneous interpretive connectivity and autonomy I desired in formulating my role and its moves?

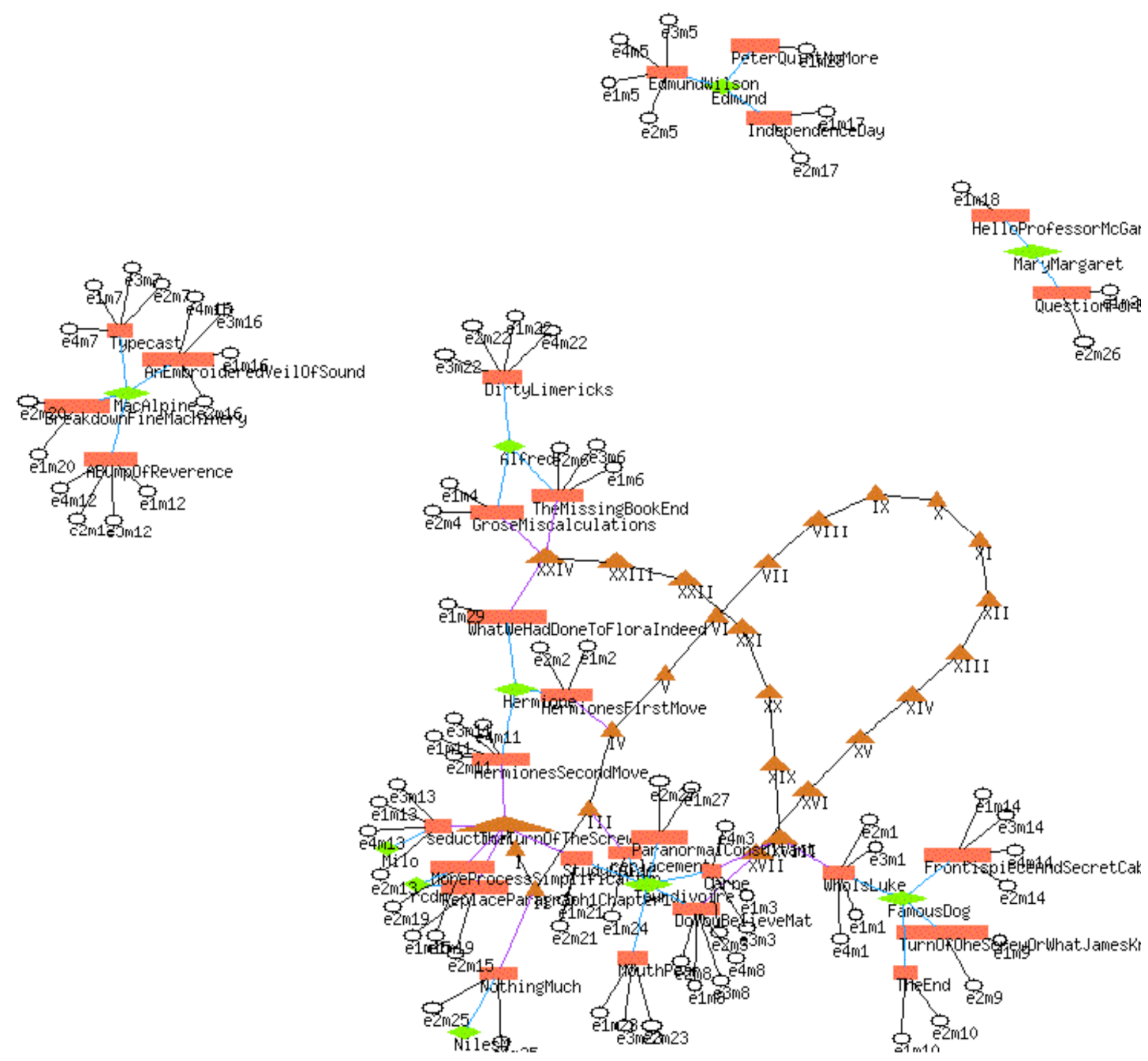

[Figure 1: GraphVis Diagram of Turn of the Screw Gameplay, Andrea Laue]

This chapter documents Ivanhoe Game-specific concepts and designs for the 
representation of subjectivity in a collaborative digital environment and contextualizes that issue with reference to pre-existing visual and computational approaches.

The task of IVANHOE's interface (ludic or otherwise) is not merely to offer a visual representation of subjectivity, but instead to create an interactive matrix in which it can be enacted and performed, and where its results are emergent at the intersection of multiple subjectivities in dialogue. But what might emergent intersubjectivity look like? How can we respond to and fuel its representation through critical engagement with texts and documents and with a community of interpreters? Computationally and in terms of design, how would such an interface function? What visual cues might players need in order to engage with the Ivanhoe Game in a manner appropriate to its own theoretical stance regarding the social and autopoietic instantiation of creative works?

These concerns may at first seem far removed from the painstaking tasks involved in the creation of digital archives and electronic editions: content modelling, text and image markup, and the development of clear and useful interfaces. In fact, the core problem facing any digital implementation of the Ivanhoe Game is identical to that which plagues the construction of more conventional electronic archives and editions: how may a rich visual environment, suitable to the expression of documentary particularity, be made to work in concert (and not just in parallel) with a rich 
computational environment, suitable to the transformation and analysis of texts?

But the case of IVANHOE is special. Because it can be figured as a game with a formalized (or formalizable) ruleset, the needs of users and their modes of interaction with the textual and spatial environment that makes up a ludic instance of IVANHOE are perhaps more explicit and easy to enumerate than the needs and actions of most users of electronic editions. Chief among these is the requirement, crucial to the playing of the game, that players be able to locate themselves and their peers - the subjective viewpoints from which all their interpretative contributions stem — at every moment of their interaction with the digital resource. This sort of interpretive mapping, if done well, should make possible new, perspectival insights into the texts at hand and into the emergent hermeneutic project of the entire playing community. Furthermore, when careful representations of the self and of subjective relations among players and the texts they both react to and transform are designed in the same visual language, a beneficial state of flow in gameplay (and therefore in critical thinking) may be achieved. The spatialized texts presented through such an interface open themselves to response in kind by players; that is, players of a subjective Ivanhoe Game might learn to manipulate, navigate, and transform the discourse field seamlessly, because they are made to feel a part of it.

Visual embodiments of subjectivity and intersubjectivity in preliminary Ivanhoe Game models come in many forms. Each of these has been designed to meet specific needs 
related to perspectival positioning:

core visualizations must be fundamentally self-centered, in that users are able to locate themselves and appreciate game-related state changes to their avatars quickly;

this self-centeredness can only exist in an obviously social context, in which meaning emerges in the comparison between personal points of view and the visualization of other perspectives;

the interface must allow for fluid and personalized regions of activity, always expressed visually and including spaces for reading, writing, evaluating, and navigating;

the gamespace must retain an emergent character in keeping with the constant transformation of a generative and collaborative hermeneutic project;

and the graphic design of game elements must facilitate a sense of response in kind in that players immediately grasp the relation of visual representation on the screen to modes of action in gameplay.

The combination of these fundamental requirements, all of which must be laid on a solid 
foundation of text-analytical functionality, challenges us as the developers of the Ivanhoe Game to push the state of the art in interface design for text-based electronic archives and annotation engines. The simple, yet highly self-conscious addition of multiple subjectivities to information visualization promises to enhance our interpretive engagement with electronic resources. In this way, IVANHOE parallels its sisterproject at the University of Virginia's SpecLab, Temporal Modelling, described in Chapter II (Drucker and Nowviskie). Our hope is that all the experimental work in tool and environment design for humanities computing now going on at SpecLab will help demonstrate the inherent hermeneutic possibilities of digital environments. We should not limit ourselves to an understanding of electronic archives and editions that excludes interpretation and scholarly engagement from their very constitution as they appear before us on a screen. These structures, and the interfaces that give access to them, need not embody mere static repositories of information.

This chapter will examine some designs for what the SpecLab team has come to call "Ultimate Ivanhoe," an implementation of the game that takes full advantage of the graphic and interactive possibilities of a spatialized and subjective discourse field, and which permits players to navigate, contribute, and respond to changes in that field using the same visual vocabulary that constitutes it. Chief among these are concepts such as the evolving avatar, a changing map of player activity that comes to represent the player himself and thereby exert a subtle influence on his gameplay by heightening selfconsciousness, and the navigable dimensional display or discourse field, a shimmering 
network of linked textual nodes that can be drawn into the foreground or pushed into the background depending on the player's interest and focus. These designs are relevant not only to the building of an Ivanhoe Game prototype, but may offer insight into new methods for editing texts in graphical environments — just as the game itself may point to new ways of analyzing and presenting existing (often underutilized) resources such as digital archives.

\section{B. Emergent Intersubjectivity}

But what do we mean by subjectivity in the context of a visual interface to textual activities? This is of course a loaded question, which I have answered already in part by figuring the content of an IVANHOE session as textual activity rather than as textual information or gathered resources. The Ivanhoe Game asks its users to create on-screen content as readily as they access it. Our latest interface sketches go so far in emphasizing the constructed nature of the textual universe that every request for a text view is explicitly represented — or in fact merely revealed — as a calling-forth along specified algorithmic or procedural lines. ${ }^{38}$ In the Ivanhoe Game, there is no text that simply is, that simply waits to be accessed by a disengaged reader. Every text view is instantiated as a result of collaborative actions among a player, his human cointerpreters (we shun the word “opponents," but some versions of IVANHOE may usefully twist collaboration into ludic competition), and the machine that responds to player actions by producing aesthetic provocations — algorithmically-derived 
visualizations — that feed back into game play in a strange, dialogical loop.

Any textual object which depends for its constitution as obviously on the physical as on the mental actions of a human user provides for a kind of performative subjectivity, a transformational condition in which personalized practices of use become critically $d e-$ formative and perhaps revelatory of embedded narratives, constellations, and meanings (McGann and Samuels). Of course any book whose cover you open opens itself up to a personalized interpretation in this way. And some of the most performative branches of textuality - gematria, Kabalistic text generation, and much of the procedural work that has come to be called ars combinatoria - offer suprisingly few opportunities for the insertion of subjectivity into their heavily proscribed generative practices (Zweig). ${ }^{39}$ Interface designs for the Ivanhoe Game attempt to make clear the degree to which every textual embodiment is the result of a series of performative and therefore interpretive acts, all done from a particular perspective, defined visually as subjective origins of display.

IVANHOE goes further, emphasizing the collaborative and constantly evolving nature of these displays as a means of rehabilitating the proscriptively algorithmic image of deformative and combinatorial work. Deformance is not, as its critics sometimes hold, a thing a machine can do without oversight and which produces an alien and decontextualized object. Instead, we wish to enact textual deformance in constant collaboration with a social group of interpreters, within the hermeneutic contexts (or 
“discourse fields") they define through their actions, interactions, and subjective positionings. The machine provides nothing more than a facilitating environment, offering computational and visual aids to scholars intimately familiar with the interpretive practices of their craft. Its responses are not answers.

So subjectivity in the Ivanhoe Game emerges as a consequence of actions and interactions, all of which derive their meaning from the self-conscious positioning of players relative to their peers and to the arranged documents in play. The simplest form of this positioning comes about when players choose roles - fictive personalities or perspectives through which to offer all their contributions to play - at the outset of a game. (This open-ended precondition is in itself a form of ars combinatoria, resulting in games in which minor characters from the novel at hand might challenge the new readings of dead literary critics and appeal for support to a weird conglomeration of printer's devils, Catholic schoolgirls, and other forces of Nature.) The requirement that players make their moves from the defined perspective of a particular role is meant to demonstrate the perspectival or subjective character of all interpretive acts; no interpretation is uninflected, disembodied, or without context. Roles do not, however, enter the game as simple substitutions for the points of view of their owners. The Ivanhoe Game's ruleset and interface emphasizes the self-consciousness of interpretation by maintaining tension between player and role. Perspectives (and therefore visual subjectivities) must remain bifurcated in order to force the kind of reflection IVANHOE is meant to promote. 
In terms of the game's basic functionality, the overlaying of roles onto a player's (sometimes-secret) self-identity requires a separation between public and private spaces. Players maintain private "role-journals," in which they document and articulate the inner logic that compels their public performance. Masks are worn in public - but not too tightly — where player moves are attributed both visually and textually to their roles and where most forms of direct engagement among players are mediated through the concept of the role. That is to say, in typical gameplay (where one textual contribution or move responds to another), players act entirely in the voices of their roles. The game does, however, provide opportunities for out-of-character interaction in the form of challenges and evaluations. Like the required role journals, special fields for challenging others' moves and evaluating the contributions of one's co-interpreters reinforce through their very presence a perspectival difference between a player and his role.

How might perspectival difference be usefully expressed in a visual environment like that called for by IVANHOE? First we must differentiate among types of perspective or points of subjectivity. The Ivanhoe Game implicitly asks players to differentiate themselves from their co-interpreters by presenting them with a space for social textual production. We might imagine a digital environment in which contributions to a shared interpretive project are unattributed, and in which the emergent text almost seems to evolve of its own accord. IVANHOE adopts the opposite strategy, marking 
individual contributions to a communal interpretive field so heavily that the field itself seems to be inflected by personality and subjective response. Each individual's game-play (or interpretive response) is therefore of necessity shaped by the actions of his peers, as he struggles to escape or gamely submits to the gravity wells of their subjective contributions. But IVANHOE's game interface makes explicit calls on players as well, as it asks them to differentiate themselves from their adopted interpretive roles. I will revisit this brand of perspectival difference in a later discussion of the evolving avatar system.

We therefore have three types of subjectivity which could support visual expression in the game: players must differentiate their acts and embodiments in the gamespace from the influencing presence of other players', must differentiate their roles' perspectives from those of other roles, and (perhaps most interestingly) must differentiate themselves as interpretive forces from the subjective, performative positions of their own adopted roles. Does this suggest that three display modes should be implemented for each player, essentially three lenses through which the discourse field might be viewed? Depending on the development of play in any game instance, each of these views might offer useful information (read: aesthetic provocation) to the player, which could feed back into his next interpretive action in an unexpected, even delightful way. But I return to the problem of defining subjectivity in the context of IVANHOE's visual matrix. Thus far we have established that subjectivity in the Ivanhoe Game emerges through embodied action and perspectival differentiation, which suggests that subjectivity, as it has 
long been figured in game studies and by theorists of virtual environments, is contingent on a participant's sense of “presence.” Is presence a necessary precondition to the generation of subjective viewpoints? Might we, in a visual and computational environment as readily as in pedagogical contexts, attempt to define concepts in question with reference to their opposites? How, in this case, might we understand the opposite of presence? The opposite of visualized subjectivity?

It's not hard to play.

There's a moment when a screen glows bright, and all those books and papers (over which you've hovered motionless, a painful curve to your neck, sporadically scribbling) seem at once to pale away and lift themselves up into icons, schematic planes for pages, squares where quartos were and rectangles now all speak octavo.

You know this instant. It's a field, now, where a desk once stood, or a blueprint in your mind that spells the library, leads you straight through unnoticed stacks to unnoticed books that house the text you do take notice of. (I knew I'd find you here.)

When it happens in the game - the smaller game, I mean - this microcosm of the Game you've been playing hard for many years — when it happens in the game, you know it as the advent of the discourse field, a manifestation, a place to be. 


\section{A Coded Message}

Insert into the discourse field now nascent around the poem you were reading - it was a

poem: “Jenny," yes? — the following passage from that other book on your desk, from

McGann's Dante Gabriel Rossetti and the Game That Must Be Lost. Careful, friend.

Note well that you transcribe here page 102 of the first edition. We're not abandoning

all convention.

“Working from Browning's monologues and Poe’s stories (especially the

hoaxes), Rossetti here produces a portrait of the inner fantasy life of a

contemporary young man, a bohemian artist. What is so remarkable about this

work - so original, as Pater would insist — is the investment its reverie makes

in realistic materials. The poem is littered with things of all kinds. It develops

an imagination of the world of the imaginary as that world is, necessarily, a

scene of desire materially reified and alienated to an extreme of facticity. The

easy conversational manner is the work's principal index of that order of the

ordinary. It carries a coded message, just as the poem is headed with a coded

title. This is not a poem about "Jenny," a prostitute, but about a young man

whose desire is drawn to her images - to her person, her imaginary self, and to

the paraphernalia that constellate around her, like the ornaments that decorate

portraitures, not least of all Rossetti’s.

"It is crucial to realize that we are led to see the whole from a Rossettian inner 
standing point, that is, from within the fantasy-space projected by the young man. It all appears to be a "real" scene, and is taken as real by the young man, as it must be: for these illusions, as a Marxian analysis would show, are precisely real. For the young man, or the poem, to see them more critically would be to break the spell of the images, to break the illusion that constitutes their reality. The man's sympathetic attitude, including his self-ironies, are not critical moves, they are blocking devices that serve to maintain the illusion of objectivity. And so it happens: unawakened from its dream of life, the poem draws the lineaments of that supreme world of illusion we like to call "the objective world," the world as will and representation, "reality.” “

$\sim$ a move made by ISP Industries, 18 August $2003 @$ 9:48:09 AM

\section{Computational Strategies}

Software engineers and the designers of virtual environments have developed a twofold approach to the problem of subjectivity, which has become increasingly vexing as realtime, networked, collaborative computing and the development of distributed suites of integrated applications grow in prevalence. The approach (as is common) splits itself along the line of the interface, and drives development on the one hand of computationally subjective structures within or in parallel to the object-oriented systems which predominate software engineering. On the other hand, VR theorists and 
designers work to create subjective interfaces for collaboration in virtual environments, interfaces that expand on the inherent perspectival sensitivity of VR to enable truly individualized experiences in which one participant's vista may differ from another's in more than physical point-of-view. I will combine an overview of the issues confronting interface designers in computer supported cooperative work with a discussion of computational subjectivity within and in resistance to the object-oriented paradigm.

The classical model informing object-oriented systems holds that the behavior and properties (sometimes called the state) of an artifact is inherent or intrinsic to itself or to the class of objects to which it belongs. All users of objects and all system components must share a single view or definition of each object, which supports a useful kind of global predictability about behavior. That is to say, a particular invocation or command made upon an object will result in the same behavior regardless of the source of the call or the contexts, classes, and states of the object and its invoker. However, situations exist in which subjective views of and calls on shared objects are desirable. Not all of these involve, as does IVANHOE, human collaboration; instead, the users may be system components or a single machine or human user at different points in time. The first workshop on subjectivity in object-oriented systems, a joint 1994 effort by IBM and Sun Microsystems, cited four such potential uses for subjective views of shared objects: 1) the construction of growing suites of applications leads to individualized needs for extrinsic properties and behaviors that cannot be derived efficiently from 
intrinsic qualities of the object; 2) multiple views of shared data have become increasingly desirable, and display routines need not always be based on intrinsic properties when, in fact, they can be written as separate applications; 3) versioning, or different states associated with a single object, could be considered as a form of temporal subjectivity if users could store a single reference without a need to update it to account for the latest (or some important past) version; and 3) debugging and software development would be enhanced by subjectivity if different perspectives on an application could be safely stored and accessed (Harrison et al.). The potential benefits of subjective orientation extend to our philosophical model of objects as well. Bent Bruun Kristensen points out that "subjective behavior would support more autonomous and evolutionary objects" because it not only enables but assumes multiple and changing states of objects, their contexts, and the users or applications that invoke them (Kristensen).

Software engineers focused on the problem of subjective enhancements to objectoriented systems are sensitive to philosophical and historical understandings of subjectivity. They appreciate that "allowing objects to present multiple views to different clients" is only a "first step towards subjectivity... True subjectivity requires that separate views contribute towards or even constitute the definition of the object" (Harrison et al. 132). This realization is in keeping with "non-classical philosophical trends that emphasize the idea that subjective perception is more than just a view filtering some objective reality," but rather that the perception "adds to and transforms 
that reality so that the world as perceived by a body of perceptive agents is more than the world in isolation" (Harrison and Ossher 143). Such concepts of subjective perception assume the recursive involution of an observer with the external objects he observes, a notion supported by our current understanding of the functioning of our senses as physical organs, and by the autopoietic theories of Maturano and Varela which have come to be central in the development of the Ivanhoe Game and its interface (Maturana and Varela).

It's not hard to play.

But when you do, you might just wonder who you are here in our field, for it's all so schematized - yet visible (near and far) - that you feel you must now be some strange new wireframe, too. No, you're not disembodied, deep inside the game. This is not, after all, your father's cyberspace.

\section{ISP Industries}

I'll help. Let's carefully insert this letter, a fiction, into the shimmer of the discourse field and link it securely to your primary move, that first foray you so cryptically called "A Coded Message."

Dear Sir, 
Thank you for your recent inquiry. Our technicians report that work proceeds on schedule, and that you may expect the experience to be available for download no later than Saturday of next week. We are pleased to have been able to incorporate the images and dimensional scan (Nell.img.zip and Nell.ds) you provided us with no measurable delay to shipment. It was fortunate that they so closely matched our pre-fabricated model — practically two sister vessels! Please don't hesitate to inform us if you have any further requests or requirements.

Sincerely,

Ivy Bannishe-K’weto

Director of Customer Service, ISP Industries

And aren't you clever, with those "sister vessels?" Better marry them (the line, your words) to the source text on which all this builds. So draw your link now, a steady streak through the discourse field, stretching from this move to Rossetti's repetitions - lines 184 and 205 - and make a goblin of the sun.

a move made by ISP Industries 18 August $2003 @$ 5:46:40 PM

\section{Subjectivity in Virtual Environments}

Interface designers who wish to incorporate subjective features into shared virtual 
environments are likewise becoming more sensitive to the relationship between vision and perception. Contrivance of an apparently objective perceptual experience is the central task confronting most branches of VR research and development; therefore, potential inconsistencies between users' internal mental models and the "self-evident," visible world (inconsistencies which in fact constitute our daily experience) have traditionally been treated as problems to be overcome. Instead, conventional VR systems "attempt to model a fundamentally objective shared reality, and to present that reality to its users as being synchronized and definitive" (Pettifer and West 170). Pettifer and West point out the fundamental unattainability of such a goal, which falters even when confronted by network lag times that prevent perfect synchronization (let alone differences in hardware and external environmental factors that contribute to subjective understanding of a communal environment). To some extent, then, designers must accept and could perhaps even exploit “ostensibly ‘shared and common’ worlds that are actually different within certain tolerances for each participant" (Pettifer and West 170).

In geographically-oriented virtual spaces — spaces that seemingly replicate real-world environments - the temptation has been to assume that simple, independentlycontrolled viewpoints which result in perspectival differences based on pitch and proximity can fully express user subjectivity. Early literature on virtual reality congratulated the technology for advancing beyond the WYSIWIS ("what you see is what I see") paradigm that dominated two-dimensional multi-user interfaces based on a 
shared desktop or whiteboard metaphor. The notion of "relaxed WYSIWIS" promoted by Stefik really amounted to basic user customization, in which a shared interface could be tailored to personal preferences through a small number of optional commands (Stefik et al.). Gareth Smith suggests that a small measure of such visual customization (choosing the shapes, visibilities, and colors of entities, for example), when coupled with the inherent perspectival nature of spatialized VR, is sufficient to meet the needs of users for subjective yet shared experience (Smith 392ff.). In this way, all users employ a broadly identical representation of a dataset and "thereby trade flexibility for the ability to collaborate in the use of the information" (Snowdon and JääAro, "How Not to Be Objective" 1.3).

Yang and Olson take a similarly moderate approach to the problem of subjectivity in VR interfaces, concentrating on issues of navigation. They identify a danger to collaboration involved in "taking subjectivity to the extreme," and warn that the destruction of shared contexts is an almost-inevitable result of viewer-dependent visual representation (Yang and Olson 2.1). Despite acknowledging tensions between collaboration and individualized perspective, the results of their study of cooperative tasking in a multi-user environment do favor the use of what they term "egocentric perspective displays." These findings about collaborative navigation are important for the development of the Ivanhoe Game in that they address enabling a variety of desirable approaches to a shared dataset: a "divide-and-conquer" approach, in which users exploit subjective views in order to complete a shared task; the practice of space- 
partitioning, in which areas of action or influence are identified and boundaries are maintained; and the assigning of different roles to individual users, from which perspectives they participate in a group task (Yang and Olson 3). The critical prerequisite to enabling personalized or subjective views that do not hinder users' ability to function collaboratively is that the system support awareness of the actions and perspectives of others.

Typical aids to perspectival understanding in shared environments have taken the form of graphical markers on the avatars of one's collaborators. In this model, avatars are represented within a common, bird's-eye view of the digital space. Headlights, view cones, or nose rays indicate the spatial orientation or gaze of the collaborators. A convergence of headlights indicates a convergence of interest (Benford, Greenhalgh et al.). Sometimes, users are given the option of switching among their own subjective perspectives, a third-person shared view of the space, and (less commonly) the private view of a peer. All of these tactics are thought to bear on the problem of navigation, which has become the chief interest of much subjective and perspectival research in collaborative virtual environments.

How, in such contexts, is navigation defined? Spence proposes a generalized framework for navigation which becomes, fundamentally, "the creation and interpretation of an internal mental model” (Spence). Wickens is more specific about the spatial processes involved in navigating a three-dimensional space, but "leaves no place for an 
internalized model of the environment such as a cognitive map.” Situation

awareness or consciousness about virtual geography or environment is merely a "byproduct of the navigation process," not an essential constituent of that process (Yang and Olson 3.1; Wickens). Fundamental disagreement about the interpretive and perspectival nature of navigation contributes to confusion in the VR literature about the role of subjectivity in the design of virtual environments.

A primary point of contention involves the extent to which situation awareness or the generation of an internal mental model depends on geographical orientation and the stability of a virtual landscape. Yang and Olson cite four basic requirements for enabling collaborative navigation, defined as information-seeking or -using among a group of mutually aware subjects: a) each participant requires an independentlycontrolled view of the environment; b) participants need the ability to converge on a common location; c) collaborative work is enhanced by the ability to understand the perspectives of one's peers; and d) participants need to remember and recognize stable spatial features of the environment, or landmarks (Yang and Olson 3). Most of these requirements fundamentally assume that the shared environment is actually (objectively) the same environment for all users. In fact, the solution to the problem of multiple perspectives (and note that it is consistently figured as a problem rather than an opportunity) is to offer some means for each user of switching seamlessly from his own view to that of a partner. Yang and Olson suggest that "it is harmful to correlate views across sites in a way that requires real-time effortful mental operation such as mental 
rotation" to appreciate the points-of-view of one's peers (Yang and Olson 5.2).

Their best solution is to minimize "effortful mental operation" by animating the transitions between visual perspectives in the form of a camera pan from one participant's point of view to another's. The feasibility of this technique demonstrates the extent to which the shared virtual environment is in fact modeled as an objectively identical, populated space. It's only the geographical positions of participants that differ; their internal mental model is presumed to remain the same.

But is cooperative work in and navigation of a shared environment possible if that environment is not modeled as an identical space for all users? What if my view of the world actually constitutes a different world from the one you inhabit? Can we still build the kind of "shared mental model" that is thought to underlie all collaboration in digital environments under these circumstances? Are there circumstances (like those at play in the Ivanhoe Game) in which "effortful mental operation" to negotiate our conflicts is desirable precisely because it reveals perspectival and cognitive differences? A shared virtual environment that acknowledges truly different subjective experiences and the degree to which these perspectives do not merely reflect but actually constitute the space of play would not only extend but also take full advantage of Benford's concept of the populated information terrain, or PIT (Benford and Snowdon). PITs, in which both users and information are explicitly embodied in the same space, are designed to "support people in working together within data as opposed to merely with data." The explicit embodiment of users in the virtual environment means that they are "not relegated to 
the status of external agents whose presence is merely implied as a side effect of their actions” (Snowdon and Jää-Aro, "A Subjective Virtual Environment for Collaborative Information Visualization” 2). This close relation between agency and embodiment is in keeping with our understanding of the constructive role of perspectival difference. The very character of the IVANHOE project implies that it is subjectivity that makes embodiment meaningful, and a producer of meaning.

There are clear advantages to be gained by modelling a virtual environment differently to different users, or even by offering different perspectives on the same space to a single user. Some of these (including the desirability of perspectival experimentation) become most evident when we imagine a data set with no apparently "natural" representational convention, unlike a CAD model of an architectural site or a VR representation of an airplane's cockpit. For abstract data sets, the most advantageous representation may depend on user idiosyncrasies or the particular tasks being undertaken by individual members of a group. The sort of landmarking advocated by Yang and Olson is perfect for geographically- or architecturally-oriented spaces in which stable landscape features not only make navigation and agreement among collaborators easier, but may (when artfully designed) help to promote certain types of actions. (The addition of virtual seating in a MOO environment in order to increase the likelihood of conversational encounters is one such example.) But appropriate visualization strategies for an abstract database may differ radically from the landscape model. Dave Snowdon and Kai-Mikael Jää-Aro rightly indicate that, although the 
underlying relations that constitute a database "may be fairly stable, their use is not — information is continually dynamically combined in new ways and the mappings from data to $3 \mathrm{D}$ space cannot be known beforehand” (Snowdon and Jää-Aro, "How Not to Be Objective” 1.3.3).

This is clearly the situation in the Ivanhoe Game, as the actions of players in creating, calling forth, and classifying new components of the discourse field and in defining and redefining the relations among these components (the central interpretive task of the game) actually constitute the evolving and emergent abstract landscape of the discourse field. The work of Snowdon and Jää-Aro in supporting subjectivity within collaborative virtual environments provides an instructive example of the directions in which an IVANHOE interface might go. Their extension of the PIT concept to allow subjective views of data and of other users addresses two central problems: the virtual environment must be "capable of displaying different representations of the same artefact to different users"; and (critical for the issue of collaboration) it must exploit "appropriate techniques for representing users to each other in the case where the users are experiencing the virtual environment in quite different ways" (Snowdon and JääAro, “A Subjective Virtual Environment for Collaborative Information Visualization” 3). These techniques and capabilities are fostered by the introduction of body-centered configuration and artifact-centered coordinates.

Body-centered configuration is a technique that allows participants to configure individual 
preferences for the behavior and display of objects they encounter in a multi-user environment. This strategy differs from relaxed WYSIWIS approaches to customization in that the users' preferences are figured as properties of their virtual embodiments, through which they themselves are "represented" to the applications that continually inspect and evaluate them as they move through an environment. No "explicit intervention" is required on the part of the user in order to specify or arrange displays as he encounters applications resident in new parts of the virtual space (Snowdon and Jää-Aro, "How Not to Be Objective" 1.4.2). User embodiments carry with them a "subjectivity flag set" which essentially generates for them new, private copies of the artifacts with which they interact. Interpreted in one sense, this approach fits perfectly with the philosophical stance of the Ivanhoe Game: it is the embodied perspective that impinges on the modeled environment to change it actively through its very gaze. In another sense, however, it runs counter to the desire of the IVANHOE interface to impress on users their own agency in shaping visualizations by forcing them to make their calls on texts and spatializations perfectly explicit.

Artifact-centered coordinate systems, on the other hand, offer a less philosophically-loaded solution to a problem facing IVANHOE's interface designers: how can we enable subjective views of a shared space so radically divergent that they in fact constitute different worlds, while still making possible intersubjective collaboration and an understanding of the convergence of these perspectives into a shared, N-dimensional universe? Snowdon and Jää-Aro suggest that such a virtual environment model the 
positions and orientations of users relative to the artifacts that populate the shared world, rather than to some absolute system of spatial coordinates. No common metric is supported; instead, each user will see his peers positioned appropriately in a landscape of his own construction. In simple terms, the task involved here is to compute a given user's awareness of a set of objects, determine which of these objects appear in the subjective world-view of his collaborator, and then place a representation (or "pseudobody") of the user in an appropriate position and gaze orientation relative to the configuration of objects that has entered the awareness of the collaborator. These computations are made using a variant of the Spatial Awareness Model introduced by Benford in 1995, in which, for each communications modality relevant to a collaborative virtual environment, an object lends itself to user awareness through its focus and nimbus (Benford, Bowers et al.). The focus of an object is a representation of its presence in virtual space, and an object's nimbus is a spatial calculation of the field of attention surrounding it. When one object's focus intersects the nimbus of another object (which may be a human user), the object enters a scope of awareness that makes interaction possible. The “Aether Extension” to Benford’s Spatial Awareness Model usefully introduces time as an additional coordinate in the awareness calculation, allowing the number or frequency of interactions and encounters with objects to figure into a computation of awareness (Sandor, Bogdan and Bowers). This extension seems critical to an Ivanhoe Game imagined not only as a prolonged collaborative project in which users might, over time, allow their focus on specific objects to dissipate and reconstitute, but also as a contextualizing activity which frequently and creatively broaches the 
historical character of the documentary record.

Despite their arguments about the inherent subjectivity of relational databases on which abstract data visualization in collaborative virtual environments can be based, Snowdon and Jää-Aro still assume some objective reality in terms of the properties of objects (Snowdon and Jää-Aro, "How Not to Be Objective” 1.3.4). For instance, display values in their body-centered model are contingent on and resident with the user's embodiment and not intrinsic to the objects that user encounters, but the fundamental classes and properties of objects underlying the display remain consistent despite the vagaries of perspective. In this way, an objective common reality for the shared virtual environment is reinforced, and perspectival display becomes mere smoke and mirrors. Can we not imagine the embodiment of more deeply embedded forms of subjectivity in IVANHOE and other networked or multi-user systems, made possible by marrying interface advances to similar advances in the computational models that underlie them?

It's not hard to play, no not now that you have your role in hand and have been mining all that data — Jenny, Jenny, Jenny — for almost a day, mining it and mulling it over until you see a pathway edging clear. There are other players now, little flashes at the peripheries, light from their spyglasses, but they don't matter much as yet. (I mean, they are your audience, but you hold no illlusions; you're peripheral to them as well.)

It's the space that influences you, you recognize, the space and being in it that calls this 
reading of the poem out. It's not a thing you could have done so well in other modes.

\section{Code Fragment}

$<!-$ Ivy, here's the bit you wanted to see. Just my first pass at it, based on the client-interview transcripts you forwarded. I think this covers all his specific environmental demands, but I'm still not happy with the overall effect. My new graphics tech, B. E. Otney, did the lion's share, and it's a little Victorian for my taste. I've put all the assets in job_1881/VR/enviro/temp/, so you can check it out for yourself. Login=“Jenny” (that's what I'm calling the babe) password="hotbed." Good luck with this guy! He seems fairly self-involved, and you know they're the hardest to please. Coffee later? - Ivan Whisk $\longrightarrow$

$<$ environment $>$

<audible $>$

$<$ interior $>$ $<$ sound type="clock" source="shelf_2" sequence="ambient" / > $<$ sound type="cage-bird"

source="misc_o0798745.241__1" sequence="post-dawn5" />

$</$ interior $>$

$<$ exterior $>$ 


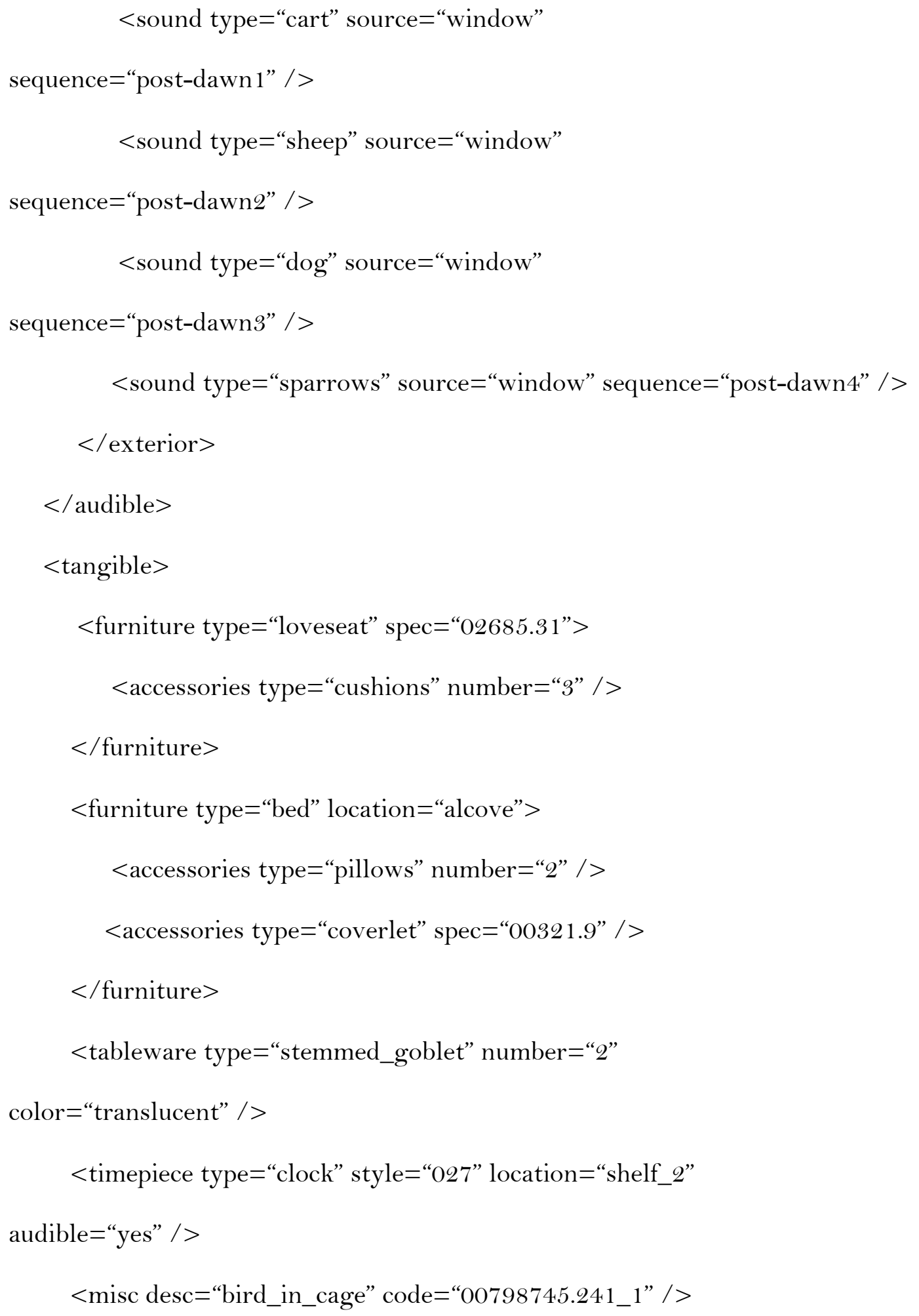




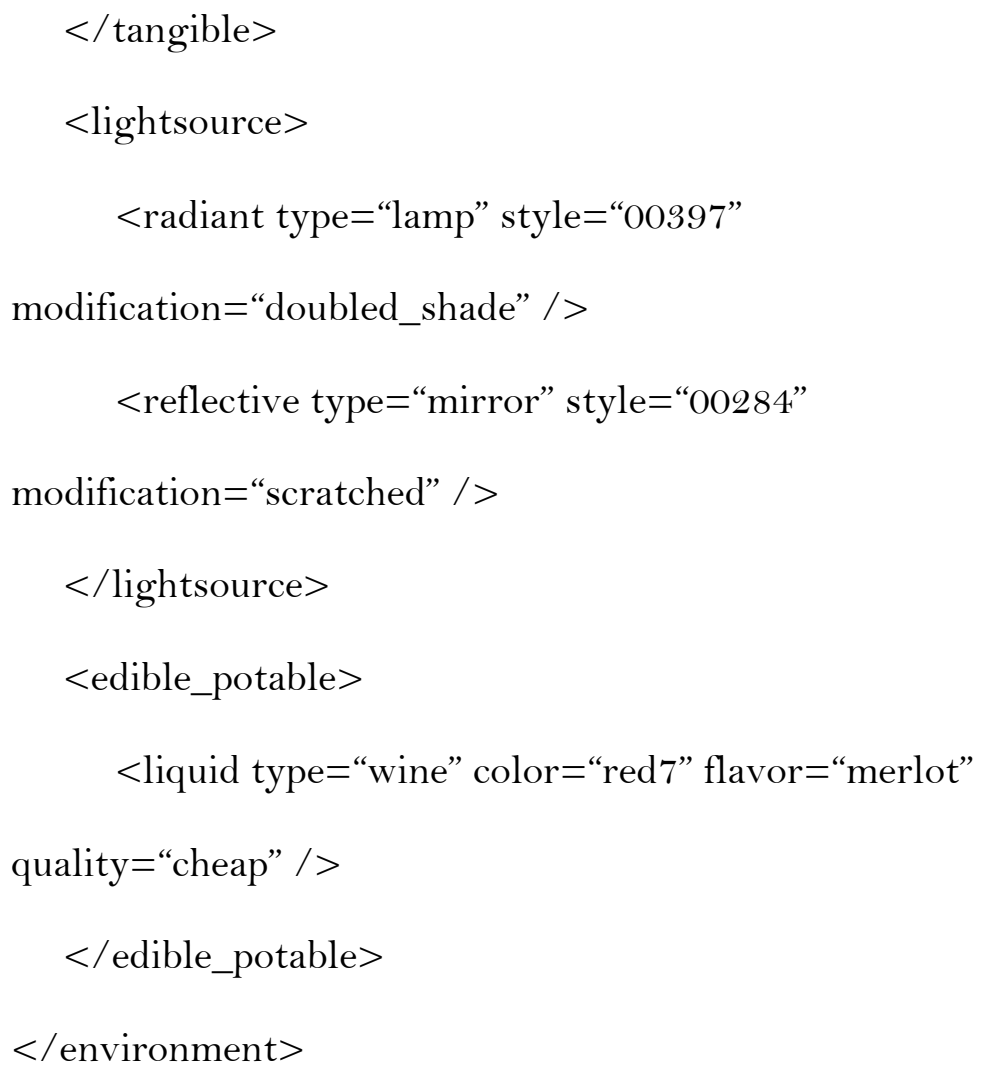

You've written it. Now watch yourself link this move with strong elastic bands to your last two moves, “A Coded Message," and "ISP Industries." Don't neglect to sketch out delicate white tendrils to tie "Code Fragment" to those lines in "Jenny" where its sounds and objects, carefully gleaned, appear. (What's fragmentary, Porlock, still can play quite whole.) 


\section{E. Subject-Oriented Programming}

Jää-Aro and Snowdon acknowledge that subjectivity is not only relevant to user interface, but could also be useful in programming environments, for example to support group programming activities without code conflicts or to enable better management of capabilities or permissions intrinsic to a particular user (Snowdon and Jää-Aro, "How Not to Be Objective" 1.3.2). In this light, Harrison and Ossher advocate a relaxed emphasis on the object in object-oriented technology in order to enable, through the exploitation of subjectivity, the development of integrated suites of applications, each of which may have different needs from and understandings of a shared collection of objects: "subject-oriented programming" (Harrison and Ossher). ${ }^{40}$ In order to understand the applicability of this concept to the Ivanhoe Game or other humanities computing projects, for "integrated suites of applications" we should read “collaborative groups of users," each of whom may formulate and express radically different and evolving ideas about a shared set of documents. The goals of subjectoriented programming articulated by software engineers are also applicable to the development of electronic archives and interfaces in this way:

Subject-Oriented Programming: "It must be possible to develop applications separately and then compose them" (Harrison and Ossher 412).

Ivanhoe Game: It must be possible for users to develop interpretations separately and then combine their expression with the collaborative project of a group. 
SO: "The separately developed applications should not need to be explicitly dependent on the other applications they are to be composed with” (Harrison and Ossher 412).

Ivanhoe: User interpretations, as they are expressed in the shared digital space through structured data and visualizations, should be able to evolve as independently of the common terminology and constraints of the established system as possible.

SO: "The composed applications might cooperate loosely or closely, and might be tightly bound for frequent, fast interaction, or be widely distributed" (Harrison and Ossher 412-13).

Ivanhoe: Close user collaboration (either with or without sharing of terminologies and typologies) should be enabled, just as private spaces and display functions are maintained.

$S O$ : "It must be possible to introduce a new application into a composition without requiring modification of the other applications, and without invalidating persistent objects already created by them... Unanticipated new applications, including new applications that serve to extend existing applications in unanticipated ways, must be supported" (Harrison and Ossher 413).

Ivanhoe: New players, player roles, interpretive shifts, and user-created documents should be able to be introduced at any time during play, without invalidating preexisting perspectives and documents. We must accept as a condition of development 
that the interpretive and creative uses to which IVANHOE software will be put are by definition unpredictable.

From the subject-oriented programming perspective, meeting these conditions requires that "no special status [be] accorded to the intrinsic properties" of objects (Harrison and Ossher 414). In other words, subjects (or the users or applications that embody subjective attitudes toward common artifacts) need only share knowledge about a given object's identity. They can separately define and operate on the object set they share, without fully understanding other subjects' object definitions and certainly without keeping their own definitions uniform over time. This means that there is no longer a "state of the object" that is in some sense true or objective. An object is no longer defined as the union of its properties and behavior, which instead reside with the interpreting subject that invokes an object-identifier. Once subjects acknowledge the existence or non-existence of an object and associate a rudimentary identifier or name with the object, they are free to extend and develop their own interpretations and perform actions or invocations without disrupting those of others.

But how, given such a separate peace, might users collaborate in or share a common view of a document or discourse field? A primary challenge to the IVANHOE development team (evident even long before the game version was played in a software environment) has been to combat the solitude or solipsism inherent in the scholarly endeavor. Humanities scholars, much more so than architects, social scientists, or 
engineers, are unaccustomed to working in teams and sometimes find it difficult to integrate their private interpretations, traditionally expressed (appropriately) in monographs, with the evolving work of a peer group. True, we attend conferences and contribute to colloquia and special-topic journal issues, but how often do we consciously and deliberately combine our perspectives on a text and produce joint interpretative work with a potentially fluid physical instantiation? In this way, IVANHOE has more in common with the pedagogical experience and with experiments in dialogue as literary criticism (viz. those of McGann and Rockwell) than with the print-based structures and institutional methodology validated by the academy. Still, this connection with team-based work, the value of which is demonstrated by current humanities-computing practices, and our conviction that the traditional tools of pedagogy can be usefully applied to the work of serious scholarship, drives us to develop an Ivanhoe Game which negotiates a rich middle-ground between contemplative isolation and cooperative creation.

To a great degree, just as in subject-oriented programming and collaborative virtual reality, this middle ground locates itself at the interface. We can understand interface as a point of interaction, whether between an application and the larger system, a user and the data underlying a particular visual manifestation, or among multiple users and the programs and displays they employ jointly and separately. In its most simple form, any shared, subjective interface is a "point of agreement between separate subjects as to the operations that are available" on an object — or more properly, on its identifier 
(Harrison and Ossher 415). The concept of agreement (which implies and enables interaction and intersubjectivity) is crucial. In essence, the classical model underlying object-oriented systems is a view of objects as seen by a single subject. This model would remain adequate if all subjects operated in isolation from one another; however, if we wish to enable interaction and collaboration, multiple subjectivities must be supported in a common virtual space.

Multiple subjects are able to interact in a shared field (or to be "composed with one another in a universe") because of that field's artfully-designed composition rule, an algorithm which amounts to an "abstract specification of the semantics of inter-subject interactions" (Harrison and Ossher 415, 23). These guidelines and constraints have little to do with the individual subjective assessments of objects that users will make and re-make throughout their work in the discourse field. Instead, they come into play when a particular operation is invoked that might provoke other users to change their interpretations about objects or to perform certain operations themselves. This, of course, is the whole point of IVANHOE as a collective interpretive experience, and such operations must be enabled in our game. Appreciating the need for flexibility that any system acknowledging subjectivity must support, Harrison and Ossher specify that, "within a subject-oriented model, there [be] freedom to craft and use different composition rules" (Harrison and Ossher 415). Chandler Sansing, a secondary-school teacher involved in the early (pre-software) testing of the Ivanhoe Game concept, concurs. He strongly advocates development of a ludic IVANHOE prototype that 
permits the instructors who may initiate games to specify the composition rules applicable to their own content and curriculum. In this way, he suggests that the Ivanhoe Game would be a superior tool (or, more properly, "environment”) to most dominant educational software applications, which present students with eternally-fixed content or, at best, a fixed set of rules and constraints which may not remain relevant to their evolving understanding of a discipline (Sansing).

I have suggested elsewhere that the Ivanhoe Game has strong natural ties to Peter Suber's thought experiment (since become a playable game itself), Nomic (Nowviskie, Ivanhoe and Game Design). The concept and preliminary set of constraints for Nomic, which Suber terms “a game of self-amendment," first appeared in Douglas Hofstadter's column in Scientific American in 1982. There, Suber addressed a central paradox in the American legislative system, in which an amendment to a law can be interpreted as applying to itself, and therefore cancel itself out or otherwise alter its own meaning (Suber).

Nomic is a game in which the design of the composition rule becomes a joint, intersubjective effort and is, in essence, the whole of the playable world. All participants begin with and share a very simple, rudimentary rule set. The rule set does nothing more than legislate, in the broadest strokes possible, the role of the rules in enforcing player interaction and the initial methods by which rules may be amended. Every socalled move made by a player is an amendment to the very rules of the game, which 
makes every participant both a player and a game designer. Even more, this game makes every player-action adhere to an existing algorithm (the prior rule set), while at the same time imagine and embody a resistance, and interpret the algorithmic consequences of any action. So Nomic is a game of pure constraint, the object of which is to think your way out of and into algorithms, algorithmically. Player moves are interpretive and active at the same time, both reflective and procedural in nature. The unstated goal of most games of Nomic is never to end, to play a continual game, delighting in the emergence of global strategies and local patterns, in the expansion of the interpretive horizon, in the relation of the parts to the whole, and (above all) in your own inventiveness in building and living in the system.

IVANHOE's development process, in which an evolving SpecLab team generates an evolving software specification and game model, bears many similarities to a prolonged session of Nomic. The mechanisms that may eventually support the implementation of those customizable rulesets Sansing suggests could, too, function like Nomic in bringing multiple perspectives and agendas in line with a single, overarching system of constraint. Shane Liesegang, a cognitive science student at the University of Virginia, is currently engaged with the IVANHOE team in an independent thesis project to develop a Nomic server, which (despite Suber's own warnings about the difficulty of computer implementation of his thought experiment) seeks to record and in a limited sense enforce the composition rules and amendments which constitute both the playing field and procedural imperative of Nomic. 
Development of an adequate composition rule (or set of interchangeable rules) to support either a subject-oriented technology or an abstract collaborative game involves a series of points of agreement among multiple subjectivities. The first of these, especially critical to the Ivanhoe Game or other electronic archives that wish to facilitate user contributions, involves object creation. Other points of agreement must converge around the nature of operations and the very nature of objects themselves.

Object creation begins when one user produces a new object (in the case of IVANHOE, this is likely to be a piece of text) and classifies it according to his own perspective (perhaps as a letter alleged to have been written by the author of the novel at hand, commenting on a particular episode in the work). In an object-oriented context, the new object would thenceforth be a letter written by the author, commenting on that passage, and all users of the object would obviously treat it as such. The various invocations they might make of the object would necessarily depend on that identity, because no other invocations would be possible, given the fixity of the object's properties and behaviors. The subject-oriented and hermeneutical nature of the Ivanhoe Game, however, specifies that users be able to treat that very object in ways different from and perhaps contradictory to those suggested by the object's creator. In other words, a second user might wish to suggest that the letter be read as a chapter in the novel, rather than as an external comment on it. 
The brand of subject-oriented programming advocated by Harrison and Ossher nicely enables this approach to object creation and modification. Two options for the integration of newly-created objects are presented. In the first, which has the advantage of conceptual simplicity, all subjects or users are required immediately to acknowledge and initialize a new object, understanding its creator's interpretation but ultimately classifying it according to their own perspectives. Since, however, understanding in collaborative contexts rarely proceeds in such an orderly fashion, the second option seems as preferable for IVANHOE as it is for distributed computing systems. In the “deferred initialization" model, a new object appears as an uninterpreted, blank slate to all users except for its creator. Only as the other users or subjects have need of the object, either because they have invoked its inclusion in a visualization or because they wish to manipulate it in some other way, are they asked to accept (albeit tentatively) its creator's interpretation or formulate their own, new classification. Deferred initialization has performance advantages in terms of both time and space requirements, but even more importantly, it "facilitates graceful introduction of new subjects that extend existing objects" - something that IVANHOE's emphasis on multiple, evolving subjectivities makes a necessity (Harrison and Ossher 417).

Further points of agreement touch on the nature of the operations or invocations available to users, and enable cooperative work with objects in spite of (or perhaps through) the varying interpretive slants given them. Clearly — as any IVANHOE session or committee meeting must indicate — " two arbitrary subjects cannot 
necessarily be composed with any expectation that they will cooperate effectively" (Harrison and Ossher 417). Limited agreement on the operations available to be called and their meaning (that is, on the actions permitted in the game space and the general results of a given action), contributes greatly toward cooperation among diverse subjects. I must be reasonably sure that my command to delete a line of poetry will function in the same way as yours in order to trust my own actions and comprehend the actions I witness. This certainty is called interface matching. Interface matching does not mean that users must share an identical workspace or that personal preferences and subjective views cannot be taken into account as actions are executed. It merely requires that some acceptable level of congruence exist in the commands available to different members of a collaborative group.

Class matching, the mechanism by which appropriate correspondences are developed among different users' interpretations of objects, is more involved. Harrison and Ossher hold that the simpler strategies for class matching dictate too great an emphasis on object and subject identity, as they require broad and centralized agreement on a set of interfaces and object-classes for the entire suite of cooperating subjects (Harrison and Ossher 417). Subjects (more particularly for the Ivanhoe Game, the critical perspectives users may wish to develop) then must be composed with that set of global definitions as constraining parameters. The very presence of multiple subjectivities, however constrained, makes this mode more suited to the needs of humanities computing than many object-oriented systems. However, the more flexible the matrix through which 
multiple users are able to match their own classification schemes with those of their peers, the greater the potential for highly diverse subjectivities to be formed and gain expression in a shared space.

One promising option for this brand of flexible matching is largely action-oriented and may be well suited to the style of interface we imagine building for the Ivanhoe Game. According to this method, when one subject manipulates an object that another subject has (through deferred initialization) not yet defined at all, the second subject may base his classification of the object on what he observes his collaborator doing with it. This may take the form of explicit class matching, in which I classify the object based on my (perhaps limited or colored) understanding of your classification scheme and the object's place in it. Alternately (or in concert), I may apply interface-based class matching, in which I make close observation of the operations you perform on the object and base my classification on them, assuming that your use of these operations, while not necessarily identical to mine, implies a system that can be mapped onto my own (Harrison and Ossher 417-20). In this way, points of agreement develop that are interface- and observation-dependent, individualized, and yet collaborative. Philosophically, this method supports the idea that the reality of the game (or discourse field, or software system, or object of academic study) is a shared construct, and that one user's interpretations depend less on the intrinsic properties of a given object than on his subjective observation of it and of other users' actions and interpretations relevant to it. Performative understanding of the type supported here is a central tenet of the 
philosophies of textuality and interpretation that give rise to IVANHOE.

Subject-oriented class matching techniques of this sort verge on the problem (or perhaps the undeniable reality) of overlapping hierarchies which plagues text encoding and markup in the context of humanities computing. With the continued development of text archives that attempt to encode creative or poetic (rather than informational) material, hierarchical markup methods like those articulated by the Oxford Text Encoding Initiative (TEI) become difficult to apply. The inadequacy generally does not become evident in marking up sentences, paragraphs, chapters, and other units that seem to express a natural hierarchy of organization - except, for instance, in those cases where a scheme for denoting page breaks may conflict with a separate scheme for marking poetic stanzas which, in a few cases, vexingly extend from the bottom of one page to the top of the next. More often, the problem manifests itself when you want to do something more patently interpretive with your markup, to express deliberately those out-of-sync creative structures which co-exist happily in the mind but less happily when parsed against an XML schema or SGML document type definition. In other words, conventional markup systems share with conventional object-oriented systems the fetters of a single, constraining hierarchy. For text markup, this hierarchy is generally informational and organizational. In object-oriented systems, the hierarchy appears as a global, inherited definition of objects and their classes. In both cases, a subject-oriented approach shows great potential in "removing the restriction of having a shared definition of the inheritance [or nesting] hierarchy" (Harrison and Ossher 426). 
Just as a subject-oriented approach to electronic text interface and encoding could permit different markup and display schemes to be applied (perhaps even concurrently) to the same document, subject-oriented systems for collaborative computing allow multiple users or subjectivities to express themselves against a shared body of objects, without damaging the interpretations of others or altering irrevocably the objects on which they act. In fact, the subjective orientation places a premium on allowing these interpretations to talk to each other in the evolving formal languages their creators articulate. The divergent, overlapping hierarchies of classification such collaborative work necessarily engenders are (in an ideal implementation) supported exactly to the degree their users desire, as points of agreement emerge based on the performative actions of the group.

These points of agreement represent a much deeper intersubjectivity than that provided by access to multiple optical perspectives (based on height or proximity, for example) in virtual reality environments. In a proposed alternative to subject-oriented approaches to programming, Shilling and Sweeney offer a similar "object-oriented paradigm exploiting views, in which an object is seen through a multiplicity of interfaces to the object. Each interface determines the visibility and sharing of operations and instance variables" (Shilling and Sweeney). In a shallow sense or for an application less conceptually tied to the notion of free interpretation than the Ivanhoe Game, such an illusion of subjectivity might suffice. But "true subjectivity," in the sense that multiple 
perceptions constitute rather than give access to the world, even (or especially) when they function in conflict, would remain elusive in a system like this. The subjectoriented approach, on the other hand, "emphasizes the ability of different subjects to form different behavioral hierarchies over objects, rather than consolidating them within a single class hierarchy" (Harrison and Ossher 426). As the Ivanhoe Game is meant to be a performative environment for the interpretive actions of multiple subjectivities, different behavioral and classificatory hierarchies must exist in concert, just as they define, for players, the textual and documentary world that (always almost) exists.

\section{F. Visualizing Interpretation}

From this review of general strategies for implementing subjectivity in digital environments, I turn now to a discussion of specific designs and concepts for the Ivanhoe Game. Although these designs - centering as they do around the interface take visual form and draw in some cases on existing practices and technologies, they embody an understanding of visualization that differs from most humanities computing projects. Visualizations in humanities computing are typically positioned as endproducts, static and algorithmically (seemingly automatically) generated artifacts representing a culmination of the interpretive processes that went into the development of a digital collection. A prime example of this is "Rossetti Spaghetti," a visual expression of links among files in the Dante Gabriel Rossetti Hypermedia Archive at the University of Virginia (Figure 2). Rossetti Spaghetti was generated by 
programmers Stephen Ramsay and Kirk Hastings using simple GraphVis algorithms, similar to those UVA doctoral candidate Andrea Laue employs in her IVANHOE-related work, and it astounds even the Archive's creators with its complexity and utility in making visible the carefully-specified yet often-obscured relationships among documents, images, and scholarly commentary in their established data structure. The image bears analysis and yields insights into the assumptions and interpretive acts that fueled the Rossetti Archive's creation. It comes, however, too late in the Rossetti development process to impact significantly the critical and interpretive work of archive-building. The content modelling phase, in which document types were defined and relational possibilities among them were opened up, is over. At best, Rossetti Spaghetti can lend us a new perspective on what already exists within the Archive, and serve as aesthetic provocation to new interpretations, which can be manifested outside of the Archive, in the form of essays, conversations, or even further visualizations. 


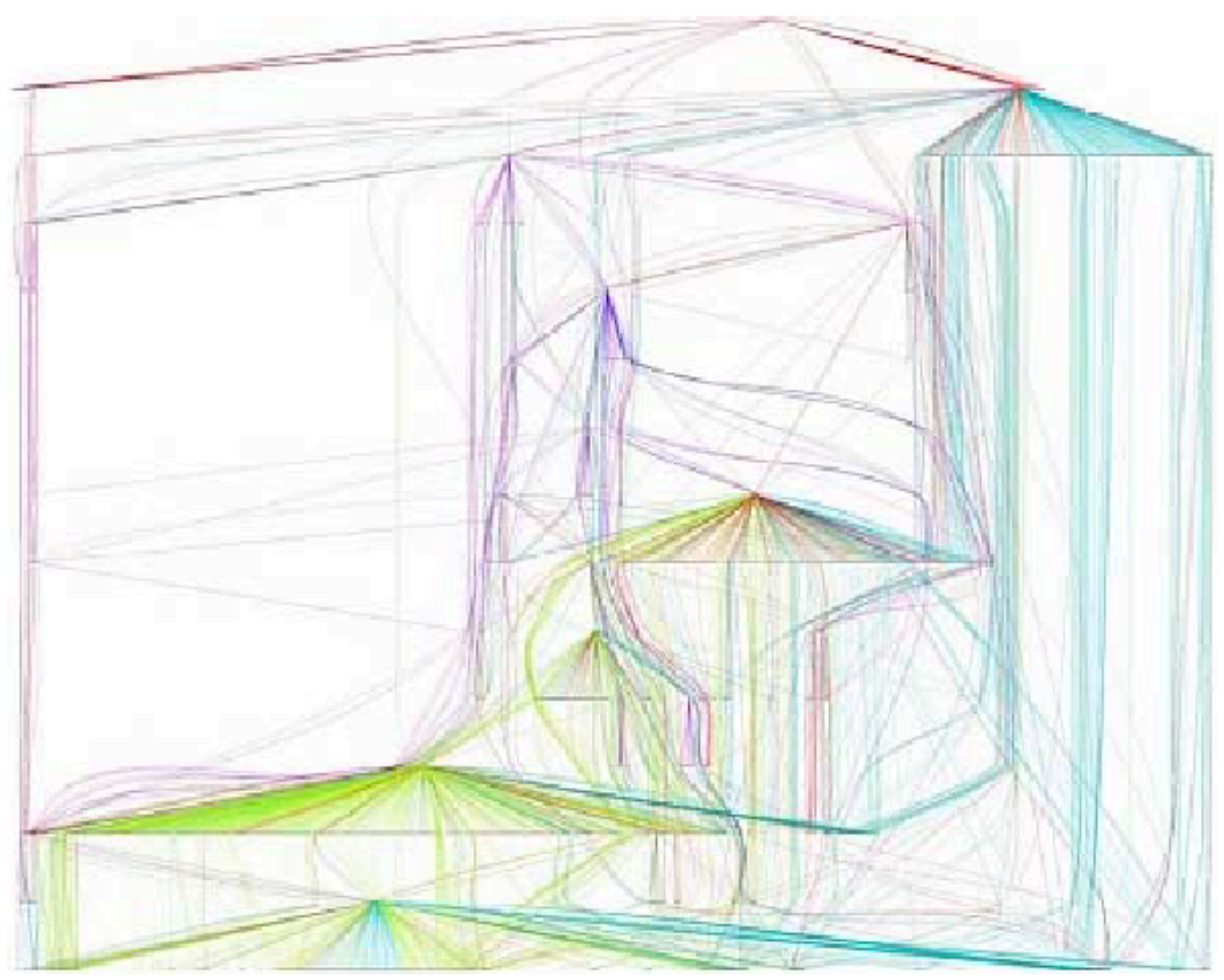

[ Figure 2. Rossetti Spaghetti $(I A T H)]$

Visualization in IVANHOE (as in the Temporal Modelling project) is meant to be part of an ongoing and environmentally internalized process of scholarship, not a snapshot of its progress or one of its external products. That is to say, every visual expression of a data structure in the Ivanhoe Game opens itself not only to interpretation by players, but also to active intervention within the very contexts in which it resides and evolves. We may still characterize these visual expressions as "aesthetic provocation," but in this case they provoke and permit interpretive responses that feed immediately into the 
computational matrix from which they emerge. Here, display is not merely generated, but also generative - of new interpretations, new data structures, new visualizations that are resident within the gamespace.

The Ivanhoe Game interface is being developed in the wake of another SpecLab project with a similar outlook on the role of the computer in enabling interpretation: Temporal Modelling (described in detail above). Temporal Modelling takes a less technologicallysophisticated but more direct approach to the involvement of visualization in content modelling, by translating user-created graphical diagrams and time-line sketches into a generalizable XML schema for temporal relations. This XML expression is a formalization of the intuitive, experimental, and even ludic brand of sketching the project enables in a paintbox-like "PlaySpace." Users may export this formalization to aid them in the development of content models for digitization and database building, or may elect to expand on their PlaySpace interpretions by working internally, in the project's graphical and text-based markup environments in tandem. No matter what the user's choice, the Temporal Modelling project demonstrates the degree to which visualization can become an integral part of a computer-aided interpretive process (Nowviskie, Temporal Modelling: Update 2003).

Similarly, IVANHOE seeks to employ advanced techniques for data visualization within the context of ongoing textual and graphic activity. This activity both occurs in and constitutes a performative space, which maps the work of multiple subjectivities in 
dialogue with each other and with a collection of documents and objects.

Furthermore, its embodiment opens itself to response by players in the very visual language in which the space itself is expressed, in order to emphasize the fluid and emergent qualities that visualization and interpretation hold in common.

Perhaps the most central of these commonalities is the shaping role of subjectivity. Despite their occasional opacity and an internal tendency in both to present themselves as having an a priori existence outside the vagaries of time and space, graphic visualization and scholarly interpretation alike are produced through and contingent on perspective. Depending on the social and aesthetic trends at work in their composition, they resist or embrace perspectival understanding to greater or lesser degrees. Regardless, IVANHOE wishes to demonstrate that the same manifestations of subjectivity evident in graphic forms can illuminate the subjectivity at work in interpretive acts, and that interpretation from explicit perspectives or roles is usefully manifested through visual techniques. I will now describe two general areas of the developing Ivanhoe Game interface that express and enable interpretive subjectivity and intersubjectivity in a ludic context, before turning once again to the wider problem in digital archive and text collection development of melding rich visual environments with rigorous computational structures.

These two areas of the Ivanhoe Game interface - the avatar system and the discourse field — involve twin challenges: how can we best represent the subjectively-understood 
textual activities of a single player or role? and how can we best represent the multiple points of view that, taken together, constitute a shared matrix of interpretive play?

It's not hard to play when you are playing with your friends, and the work you're doing here to wring out their points of view has opened up your own in some surprising ways.

She styles herself quiotl, which makes no real sense to you, and you are following her moves to find the pattern that you know is there, against which you must already weave. Old leonardo's not so tough, though he's sure to twist the warp and woof a bit before it's over. (And the metaphor, if tortured, still is apt.)

You turn back to "Jenny" now. Is this the thing to provoke them both?

Let the thoughts pass, an empty cloud!

Suppose I were to think aloud,-

What if to her all this were said?

Why, as a volume seldom read

Being opened halfway shuts again,

So might the pages of her brain

Be parted at such words, and thence

Close back upon the dusty sense.

For is there hue or shape defin'd

In Jenny's desecrated mind,

Where all contagious currents meet,

A Lethe of the middle street? 
Nay, it reflects not any face,

Nor sound is in its sluggish pace,

But as they coil those eddies clot,

And night and day remember not.

\section{Bring Out Number Weight and Measure in a Year of Dearth}

Link the following to quiotl's "heat" move (which reads "Replace line 14 with this new line 14: When the hot arm makes the waist hot"), leonardo's "Second Move" (Dear Mr. Buchanan... Blake's diabolic aphorism could not be more aptly applied than to the question you raise in your letter...) and to lines 163-170 of the source text.

From: Noah Kevin Bitwyse < bitwyse@ispindustries.com>

Date: Wednesday Aug 20, 2103 08:47:56 AM US/Eastern

To: B. E. Otney <otney@ispindustries.com>

Cc: Ivy Bannishe-K’weto <ivy@ispindustries.com>, Ivan

Whisk<whisk@ispindustries.com>

Subject: Re: heat

Otney, I understand you're doing graphics for Ivan on this "Jenny" project.

We're running into some trouble down here in Sensorimotor Apps. Maybe you can cast some light. 
The issue is that heat transfer (ie. from the user's body to the vessel) is causing some artifacting. We can't figure out if the problem is in the vessel's "body heat" programming or if it's a graphical glitch, but whenever - for instance - the user's hot arm makes the vessel's waist hot, we're getting a weird cycling of day/night ambient light from the window, and it's completely throwing the vessel off. She's basically not sure whether to wake up or drift off. And hey, I don't need to tell you that the client isn't paying for her to sleep!

Could you check into this ASAP? What I specifically need are your ambient numbers, some data on how the graphics handle weight (it could possibly be the pressure on the vessel and not heat transfer), and whatever probability measure for artifacting you're working from. - Noah

$\sim$ a move made by ISP Industries 20 August $2003 @$ 8:47:56 AM

\section{G. Evolving Avatars}

The first challenge (how to represent the subjectively-understood textual activities of a single player or role) is, in part, addressed through the Ivanhoe Game's novel approach to player avatars. Generally speaking, avatars are the embodiments of players or player-roles in games and virtual environments, and are usually thought of as "soul-less bodies for which the user acts as mind” (Sengers, Penny and Smith). We know them 
through non-digital manifestations (the top hat in a Monopoly game is a simple, tangible avatar) and through electronic manifestations (the voluptuous Lara Croft character of the Tomb Raider franchise is a much-discussed digital avatar) (Flanagan). An avatar typically functions as a placeholder or token that represents the position of the player in a virtual space. While it is grossly manipulable in the sense that it may be moved about or, particularly in digital role-playing games, clothed and equipped like a paper doll, the avatar is a fundamentally stable representation of the player - easily recognized, largely unchanging in form. Only recently has the game design community begun to question this stability. A yet-unreleased digital fantasy RPG (Big Blue Box's Fable) promises that a player's moral and physical actions throughout the course of the game will irrevocably alter the appearance of his avatar. Excessive sword-fighting, for instance, will result in the over-development of the avatar's arms, while an unhealthy interest in the occult will result in a pale and haunted aspect. Avatar appearance in turn will feed into the responses and actions of non-player characters in the game, provoking fear or admiration among a village's inhabitants in some cases, closing doors or opening up plot-lines in others. The game's development code-name, Project Ego, suggests the importance of this feature in the minds of its creators and in the evolution of the digital role-playing genre (Molyneux, Carter and Carter).

Clearly, the Ivanhoe Game is meant to be a kind of "project ego," in which the force of player decisions, through the subjective lens of the role and the intersubjective lens of the interpretive community, colors and even shapes the world of play. Like Fable, our 
game advances the concept of the avatar by making its physical manifestation both a product of and an instigator to player action, always figured as an embodiment of a self made real through activity in a social and artifactual universe. Our "evolving avatar system" therefore runs counter to the longstanding concept of the avatar as a stable representation of the player and as a mere token or placeholder in a virtual space. In fact, instability is the hallmark of this system, in which each player's avatar changes from move to move and serves as a dynamic map of his actions in the gamespace.

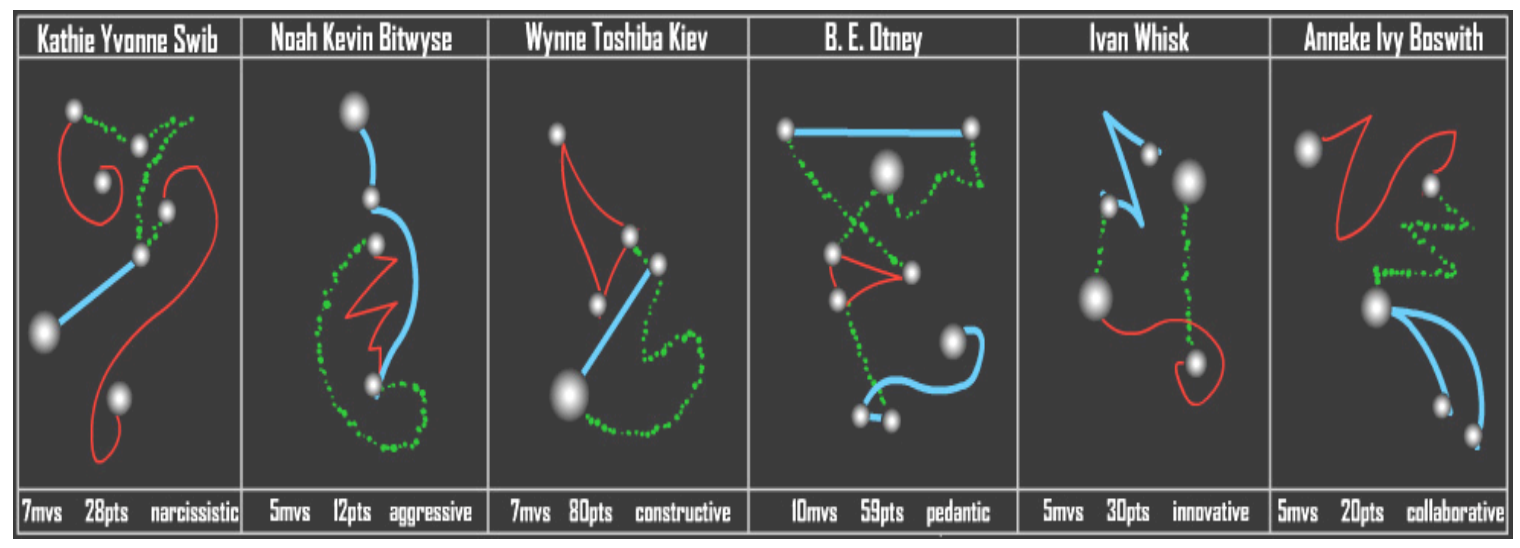

\section{[ Figure 3. Evolving Avatars ]}

In the design sketch shown here, silver nodes representing the player's textual contributions are joined by lines representing links or relationships expressed in the discourse field (Figure 3). The size of the nodes indicates the length of the textual contribution, while the colors and qualities of the lines correspond to a typology of linking moves either established by each player individually or agreed upon by the group. An animated sketch, available on the SpecLab website, shows how the form of an 
avatar morphs over time, depending on the actions of the player or role to which it is bound (Nowviskie, Future Ivanhoe Visualizations: Evolving Avatar). The metric and style of representation in play matter very little, and the exact specifications for the activities to be modeled are likewise adjustable, so long as the avatars are made to depict gameplay consistently and in an aesthetically provocative way. (Of course, a style of avatar representation that matches other data visualizations in the game would best promote the sense of response-in-kind that I earlier posited as a goal of interaction in the IVANHOE interface.)

Like Rossetti Spaghetti, an Ivanhoe Game avatar will emerge as a visualization of marked data, perhaps even taking form as a similar network of nodes and connections. This kind of information visualization differs from the Rossetti example, however, in two important ways. First, while it is meant to be publicly visible, an Ivanhoe Game avatar is personally-directed. Each avatar is to be understood by its player as a manifestation of selfhood and a reflection of personal behavior in a social space — an ego projection. The abstract, non-anthropomorphic form of the avatar helps to emphasize that avatars are not simple extensions of users or evidence of a user's wholesale transportation into virtual space. Instead, the Ivanhoe Game design participates in what Simon Penny has called a "semi-autonomous avatar paradigm," in which the avatar is not meant directly to represent the user, but rather to be seen as "the part of the system which is intimately connected to the user" (Penny, Smith and Sengers 22). Semi-autonomous avatars are revelatory, through unexpected behaviors or 
responsive physical manifestations, of the inner workings of the computational and social structures in which they reside. They partner with users and ask to be understood as separate but connected entities. Fundamentally, the avatar intrudes into gameplay as an interpreted representation — interpreted by the system, by the player, and by the player's peers.

The close proximity, in our Ivanhoe Game interface sketches, of other players' avatars encourages comparison and reflection, leading to a greater understanding of the relevance of visualization to play and embodied action. The design reproduced here goes so far as to propose addition of some subjective or qualitative verbal assessment of the style of play each avatar comes to represent, indicating gameplay that is "aggressive," for instance, or "charitable." These assessments could be generated algorithmically through analysis of the avatar's visual features, solicited from other players, or filtered from the prose evaluations of peer-moves the Ivanhoe Game requires. The addition of qualitative assessments in textual form to the graphic assessment that is the avatar is meant to emphasize players' ability to "read" the images that come to represent their roles and those of their co-interpreters. Like everything else in the gamespace, player avatars are meant to be interpreted subjectively and socially, no matter how seemingly "objective" their mapping of player action may appear.

The other way in which Ivanhoe Game avatars differ from data visualizations like 
Rossetti Spaghetti involves their embedding in a dynamic space open to transformation with every user action. While, at any given moment in gameplay, the avatars do function like snapshots, showing a frozen view of the community's interpretive project, they are best understood as evolving or emergent reflections. While they do not enable the kind of direct action typical to most game avatars (running, jumping, shooting), Ivanhoe Game avatars participate more actively than most in the constitution of the digital space by representing it to players and reorienting it toward their subjective points of view or their roles. In this way, they are reminiscent of Michael Mateas' “subjective avatar” artwork, likewise meant to challenge the conventional notion of the avatar as a "passive puppet, providing unmediated agency within the virtual world" (Mateas, "Expressive AI: A Hybrid Art and Science Practice”). Mateas' avatars are designed to emphasize the mediation of virtual experiences, by filtering "objective" environments and occurrences through an "autonomous personality model which reacts to events in the world and maintains an emotional state and narrative context relative to these events." The individualized state of the avatar becomes a lens through which the world is modeled, a "magic pair of glasses which allows the participant to inhabit an alien subjective position" (Mateas, "Expressive AI: A Hybrid Art and Science Practice” 147). Mateas characterizes all of his work in the field he calls "expressive AI" as having more to do with artistic performance and cultural production than with traditional concerns of the artificial intelligence community, such as task competence and objective measurement (Mateas, "Expressive AI: A Hybrid Art and Science Practice” 149). But the performance, in his model, is all on the part of the 
expressive AI artist, the programmer of the subjective avatar. Users who inhabit the avatar have some level of agency in navigating the virtual world, but they are fundamentally members of an audience, not performers themselves: "the avatar becomes an additional artistic resource for authorial expression" (Mateas, "Not Your Grandmother's Game: AI-Based Art and Entertainment”). In contrast, Ivanhoe Game players are able to respond directly to information gleaned from their avatars and, in a desire to re-shape their physical manifestations in the gamespace, may engage in shaping that very space in ways which would not have otherwise occurred to them.

Through its emphasis on shaping and re-shaping, our avatar design complements the work of Kristine Deray in modelling notational avatars for collaborative work in information spaces. Deray confronts problems of analysis and display by embodying information in her avatars just as the Ivanhoe Game does. She proposes an avatar design that represents gained knowledge about the contents and organization of a database in the "geometry and structure of the avatar visualized at the interface. In this manner, the design and form of the avatar functions as a content analysis tool representing interaction between users and an information space” (Deray). Different visible patterns in the avatars emerge based on rules interpreting user actions and interactions. These rules, however, apply to activities largely limited to searching and navigating a stable database, whereas Ivanhoe Game avatars map more creative and subjective interaction with an evolving space, centering around the creation of new material and the relationships that individual interpreters forge among documents and 
each other. Where Deray's avatars are outward-focused, indicating learned information about the location of material and the movement of peers in order to facilitate navigation, our avatars demand introspection and become ultimately less about the shape of the gamespace than about the shape of the player's role.

Player avatars are one strategy for visualizing and even embodying subjectivity in the Ivanhoe game. The degree to which these avatars are best understood in juxtaposition with each other demonstrates the centrality of the concept of intersubjectivity to the wider IVANHOE project. The Ivanhoe Game is designed for collaborative play and its interpretive products are those of a community of users. This brings us back to the second challenge outlined above: how can the Ivanhoe Game best represent multiple points of view constituting — like scholarship generally — a shared field of interpretive play?

\section{H. Intersubjective Discourse Fields}

We call the "populated information terrain" in which both IVANHOE documents and users are embodied a discourse field. This term emphasizes the dialogic nature of action in the space, where texts and images become more than static research resources to be accessed in the aid of scholarship that happens elsewhere. IVANHOE players, through their work in creating, interpreting, classifying, and linking bits of data, place documents in conversation as surely as they position their own roles within cooperative or competitive matrices. While the Ivanhoe Game itself exists as something of a 
hothouse, this conversation is meant to invoke a wider, wilder range of scholarship possible in the context of a rich documentary environment. Therefore the discourse field, as it is visually expressed, should imply extent beyond the objects (texts, sounds, and images, all centering around the primary document in play) that have been defined and brought into the game by players. The field itself is meant to be understood as a realm of possibility, which could support any document a user might wish to call or create and which is capable of representing any constellation of objects, references, and links. This is not to say that the discourse field is completely nebulous, either conceptually or in its visual manifestation on the screen. The practice of algorithmic calling-forth which the Ivanhoe Game demands as part of bringing any piece of data into view demonstrates the procedural constraint inherent in all textual spaces, in archives as surely as in leaves and gatherings. Although the discourse field seems unbounded in scope, it is still constrained by our inherited procedures for defining and interpreting documents and works, and the expectations those procedures engender. Fostering awareness of the constraints we often unconsciously accept as a precondition of interpretation is a central goal of IVANHOE.

A further mode of constraint at work in the discourse field is perspective. The interface (which in this case should be understood as more than the configuration of tools available to a user, and instead as all the graphic structures through which data is presented and made accessible) is made as personalizable as possible. This means that the visual organization of the space is relative to each player's role, and players may 
construct arrays of documents and reposition discourse field elements in patterns most suitable to their own interpretive enterprises. These fluid and idiosyncratic organizational schemes would be, in a primary mode, visible to their creators' roles alone, despite the fact that the discourse field remains a shared space in which many roles are working and - because they are embodied within the information terrain actually have presence. Snowdon's notion of artifact-based configuration (as outlined above) is one option for making this openness to individually-defined patterns of constraint possible, without precluding the active presence of other users within a personalized view (Snowdon and Jää-Aro, “A Subjective Virtual Environment for Collaborative Information Visualization”). In this way, the Ivanhoe Game could allow users to define their own dimensions for display, just as they define (or inherit) the “dementians” through which literary interpretation always occurs (McGann). The difference here is that these dementians are made explicit through action in a visual field, in which relational and transformative performance is necessarily defined along user-created axes. Perspective, so often thought of as an abstract quality at work in scholarship, is here made visible.

But just as our evolving avatars become most meaningful in comparison or dialogue with each other, so do the visually-manifested interpretive assumptions and hypotheses of individual players of the Ivanhoe Game. In the case of avatars, comparison is a simple matter so long as a shared set of parameters for display can be applied to the most basic activities of gameplay: move- and link-making. But if the discourse field 
really enables multiple free and subjective organizational schemes to be laid over a shared data set, how is it possible that these schemes be reconciled? How is it even possible that one player be made to appreciate the relation of his own scheme to that of another user?

We require visual and computational strategies, working in tandem, in order to address these problems. One tactic would be to adapt Yang's practice of transitioning shifts in point-of-view from one player to another via graphic animation (Yang and Olson). Imagine watching your careful configuration of discourse-field objects slowly morph into an unexpected shape, representing (and perhaps, in its motion, revealing) new interpretive hypotheses or assumptions as individual documents slide fluidly into different user-specified positions on the screen. It would be possible to run this perspectival morph once (even repeatedly), for careful comparison between two roles, or as a long sequence, showing the discourse field configurations in use by all players of the game. A temporal element, too, could be introduced, as players compare their own prior organizational schemes with current ones, or run a view-morphing animation that accounts for multiple subjectivities over time. This perhaps jarring shift in subjectivity might even become a regular, provocative element of gameplay: a random occurrence, or a strategy to be employed by players eager to understand the hidden connections between their own work and that of their co-interpreters.

Another possibility involves treating user-defined interpretive dimensions/dementians 
in the same way that subject-oriented programming handles class matching among users with radically different notions of object classes and properties (Harrison and Ossher $417 \mathrm{ff}$.). Would it be possible to use class matching techniques to synchronize in tolerable ways the visual dimensions either defined by users explicitly as they organize their views of the discourse field or which emerge implicitly from similarities in their patterns of action? In other words, could the similarities between individual interpretive views that are a necessary component of computational comparison arise, as in interface-based class matching, as a result of observation - either on the part of the machine or its users - of the practices of gameplay? Or could users be asked to define and compare their own interpretive strategies against those of their peers, as they see both of them manifested within a similar visual matrix and constraining ruleset? In this case, the tactics required by explicit class matching could become part of the consciousness-raising project of the Ivanhoe Game, in which all the processes of gameplay (calling documents, arranging views, articulating roles, etc.) contribute to making players aware of their own agency as interpreters. Either scenario would permit the use of transparent overlays of individual views, carefully coordinated to share axes of interpretation, in order to construct a communal view representing the hermeneutic project of the entire playing community.

The construction of such overlaid views of the discourse field makes it possible for the Ivanhoe Game to offer as clear a visual expression of communal work as it does of the inner standing point of an individual player. The graphic manifestation of an inner 
standing point, or a subjective origin of both interpretation and display, is an important contribution of IVANHOE’s sister-project, Temporal Modelling, to visualization in the humanities. The notion is relevant here, too. In Temporal Modelling, users define an inner standing point for each timeline they construct as an interpretive hypothesis about human perception of history. Using a specially-designed tool, the nowslider, they position that standing point (perhaps understood as one person's or group's perspective on a series of events) at different intervals along the line, using its motion to reveal an evolving subjectivity. That is, they explicitly define a subjective view and configure a visualization around it and its perspective (Nowviskie, “Temporal Modelling: Visualizing Temporal Relations for Humanities Scholarship (Part Two: Composition Tool Design)”). In contrast, the Ivanhoe Game allows each user to define his own inner standing point implicitly as his role is articulated through textual activity in the course of cooperative play. Jointly, all these individual perspectives converge in contributing to a shared interpretive stance vis-a-vis the central document at hand and its procedurally-specified discourse field. This shared stance will change from game to game (even from moment to moment), constituting an important, visually manifested record of one community's engagement with a scholarly problem. Saved records make it possible to compare these defined dementians across several games using the same core text or shared genre, focus, or interpretive task, in order to reveal embedded assumptions that emerge when different groups approach similar issues. Likewise, an individual user could compare his own responses (mediated through a role common to several games) to a variety of texts and interpretive 
situations. This makes the navigable dimensional display of the IVANHOE

discourse field as reflective on a large scale as evolving player avatars are on a small scale.

\section{IVANHOE and Digital Scholarship}

I return now to a question with which I opened this essay: "What visual cues might players need in order to engage with the Ivanhoe Game in a manner appropriate to its own theoretical stance regarding the social and autopoietic instantiation of creative works?" This work - partly speculative and partly based on our own prototypes and research in interface and database design — has addressed those aspects of that central theoretical stance related to subjectivity and intersubjectivity. The idea has been to organize both the computational structure of the game (for example, through a judicious use of subject-oriented programming) and the game's visual structure, through features like evolving avatars and comparative discourse field displays, in such a manner as to emphasize that IVANHOE hinges on the free play of interpretation even within environments that normally — unfortunately — seem fixed and objective, like a digital archive or algorithmically-generated data display. Furthermore, these proposed designs assume that interpretation is always of subjective origin, and they demonstrate that such subjectivity can take visual, iterative, emergent form, feeding back into the computational matrix from which it has been coaxed. The ludic attitude of the Ivanhoe Game toward documents and subjectivities in juxtaposition is not as far-removed from 
the conventional scholarly arena as it might appear. IVANHOE is, at least, a thought experiment in the application of gamelike and intersubjective features to digital archives and collaborative work. At its best, we can see IVANHOE and its ludic subset as revelatory of the inherent nature of networked scholarship. Digital resources are not static, uninflected spaces in which information is stored. The Ivanhoe Game suggests that subjective interpretation, not objective access, is always-already the primary activity of a user of any discursive resource or digital environment so imagined.

Imagine, then, applying the game's interface-design strategies to existing, hierarchically-organized digital collections such as the Blake or Rossetti Archives. What role might there be among them for a strong subjective emphasis and our brand of openness to interpretation as an integral part of the constitution of a digital resource? That is, how might the structures that support these archives, and the methods that display them on a screen, be opened up to users in such a way that every encounter with a resource becomes transformative and constructive, rather than informative and - despite the early rhetoric of interactivity surrounding hypermedia — fundamentally passive?

My ongoing work in designing and now redesigning the interface to the Rossetti Archive addresses these issues (see Appendix $\mathrm{C}$ below). In brief, I see a subjective and intersubjective approach as critical to making the case that digital resources (like bibliographical resources generally) should be understood differently by the academy. We need to show that these objects and collections open themselves up to interpretive 
scholarship in their very assembly and structure, and that they can be made more fluid and responsive to the activities of scholarship — that work within them can be less like navigating an archive and more like building an archive, less like using a scholarly edition than like constructing one. This fundamental and explicit shift in the role of users of digital resources - toward the performative, reflective, subjective, and constructive activities that characterize scholarship generally — should be highly salutary to the field of humanities computing. The relevance of visualization and text technology to the academy at large may depend on our ability to bring emergent intersubjectivity into play.

$$
\text { | And, you know, it's not so hard at all. }
$$

\section{Licensing Device}

Link the following, printed on a small cardboard box, to this sentence in leonardo's third move: ("The Valentinian sonnet that so troubles you is a licensing device that opens wilderness territories, an imaginative version of what the Americans are doing these days to open their westward lands.") Link it to the discussion in this very dissertation (Did you think I wasn't reading along? Were you?) on "deferred initialization." Link it also to lines 135-138 in “Jenny," your old source text, a thing now burning at the center like a star, casting shadows everywhere:

Jenny, you know the city now.

A child can tell the tale there, how

Some things which are not yet enroll'd 
In market-lists are bought and sold.
Attention: Mr. D. G. Rossetti, Esq. -

Only a few bureaucratic details now stand between you and your desired experience! As you are no doubt aware, Inner Standing-Point Industries must comply with UN regulations concerning the use and disposal of anthropomorphic vessels and their cognitive encoding systems. With your purchase of expo01881-2 (project code-name: Jenny), that responsibility passes formally to you.

The talented team here at ISP has pioneered a new method of transferring and regulating the use of our vessels and their “personalities," by further exploiting the nanotechnology that is so integral to our success in producing high-quality experiences for valued customers like you. In this package, you will find a small vial of pleasant-tasting liquid labeled "licensing device." We ask that you ingest the liquid no more than 24 hours before you intend to commence your new experience. Compliance with this procedure will ensure that exp001881-2 is licensed to you (or more properly, to your DNA) as sole owner.

Enjoy! 


\section{IMAGINARY SOLUTION \#3: The Somehow May Be Thishow}

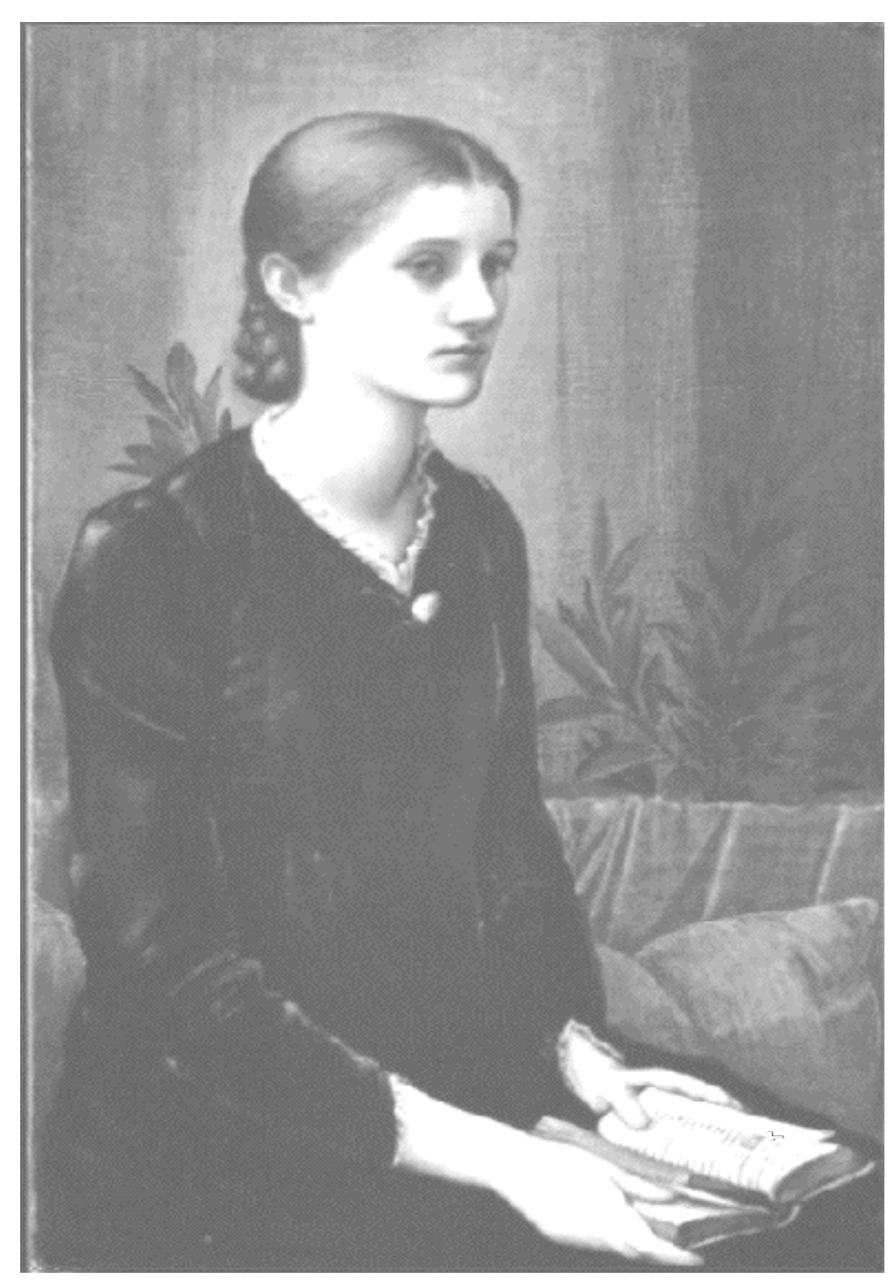

In Edward Burne-Jones's portrait of Caroline Fitzgerald (1884), the clue to the young model's oddly candid yet withdrawn gaze appears to lie in the undisclosed subject of her book... The admiration of this young and aspiring scholar-poet for Robert Browning is sadly prophetic. Like Browning's Pompilia and his Duke of Ferrara's last Duchess, Caroline is the victim of a domestic tragedy that would make the subject of a dark but fascinating dramatic monologue... Caroline Fitzgerald's tenuous but intriguing connection with Robert Browning, who dies the year she dedicates her poems to him, invites us to compare BurneJones's portrait with Browning's art of writing dramatic monologues... Since BurneJones's model is gazing fixedly at an object in space, and the observer is looking at her from a different angle, we seem to be eavesdropping on her, gazing at her through a keyhole. What we see is relative to our position in the room. Sharing the premise of some dramatic monologues, the portrait refuses to provide a privileged angle from which to observe its subject. Nor is it any longer possible, as in an epic poem, for every observer to see everything. Because our eye is no longer placed at a privileged focal point, as in the perspectival painting of the Renaissance, where everything converges on a single observer, the portrait's meaning appears to be the total sum of all possible views we might take of it. But to view the picture so inclusively, an observer would have to perform the visual equivalent of reading a series of monologues like Browning's The Ring and the Book... Like a monologue or candid snapshot, BurneJones's portrait recognizes that each viewing is a private act: there is no unique or privileged centre.

W. David Shaw, "Edward Burne-Jones and Pre-Raphaelite Melancholy.” University of Toronto Quarterly, 66:2 (Spring 1997) 
11 algebraic representation of some Robert Brownings, some historical and some purely historiographical "men and women," and the great work in which they are all bound up: The Ring and the Book. Morse code, effectively a chart of compromise, interpretation, and the subjective positionings of poet and poetic character, looks like this

B...@< cB@[ $[(\mathbf{a} @ \mathbf{x})+(\mathbf{b} @ \mathbf{x})+(\mathbf{c} @ \mathbf{x}) \ldots]>$

and is always, always left as an exercise to the reader. ${ }^{41}$

Mastering his matrix, Robert Browning satisfied all preconditions of an Ivanhoe Game. The rest of us (and not least Caroline) would like to play along. How to set the stage?

A discourse field, densely populated. The temporal axis: 1698 Romana Homicidiorum $\sim 1860$ Do you see this square old yellow Book? (I.33) 1868 The Ring and the Book 1884 somber She sits for Burne-Jones 1889 Venetia Victrix $\sim 1915$ Hodell facsimile edition 1968 B...@<cB@ $[(\mathrm{a} @ \mathrm{x})+(\mathrm{b} @ \mathrm{x})+(\mathrm{c} @ \mathrm{x}) . .]>.\sim 2004$ and yes, the somehow may be thishow (I.707). 
Against it all the things.

Eighteen pamphlets and a secondary source, a diagram or stemma, but this must be a living library in that they all fall loose when touched, slide from their moorings, hover,

hum between

book. A book, comfort us,

The thing's

touch and

But the pages

there's our

in a word

avatars, where

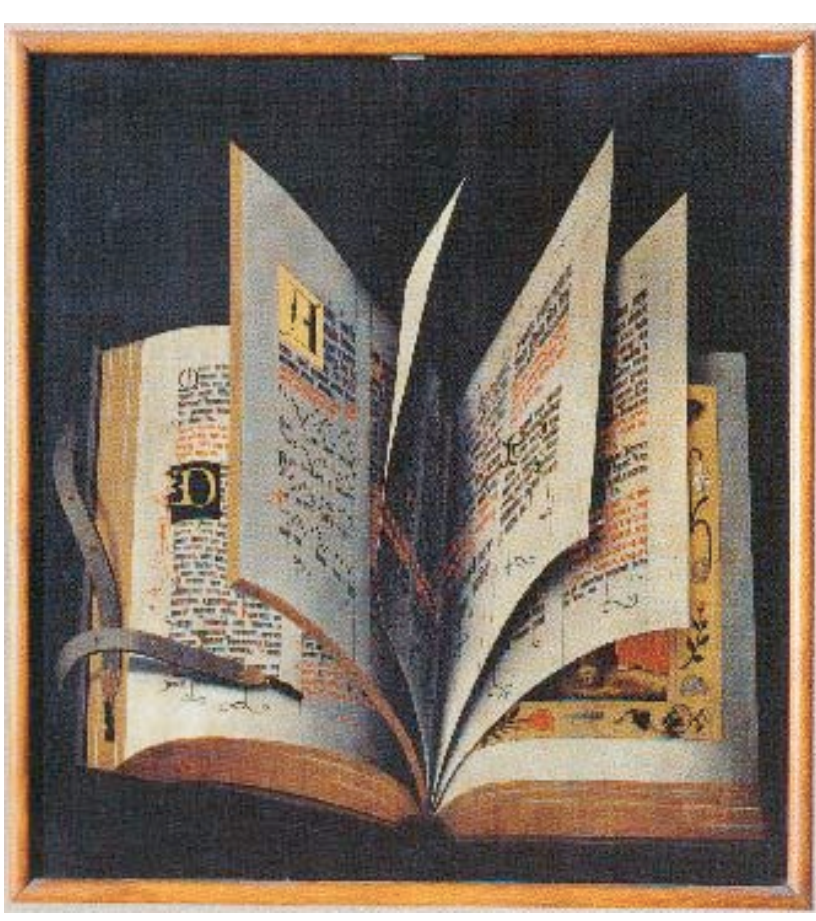

the chart and the

it's true, may

unmoored, you know

restorative / I' the

sight (I.89-90).

aren't the point, for

Count / Charactered

(I.188-9), a set of

all of us are taking

shape: Half-Rome and still the other half and tertium quiddity. Guido $\sim$ Pompilia, Caponsacchi. Archangeli Bottinius \& Browning(s) \& the Pope. Morse, Caroline, Shaw, the book her book (this book). Burne-Jones, paint our portraits, keyhole views!

For how else know we save by worth of word? (I.837) 
One answer holds: beyond such laws lie other "recognized machines" (I.1110).

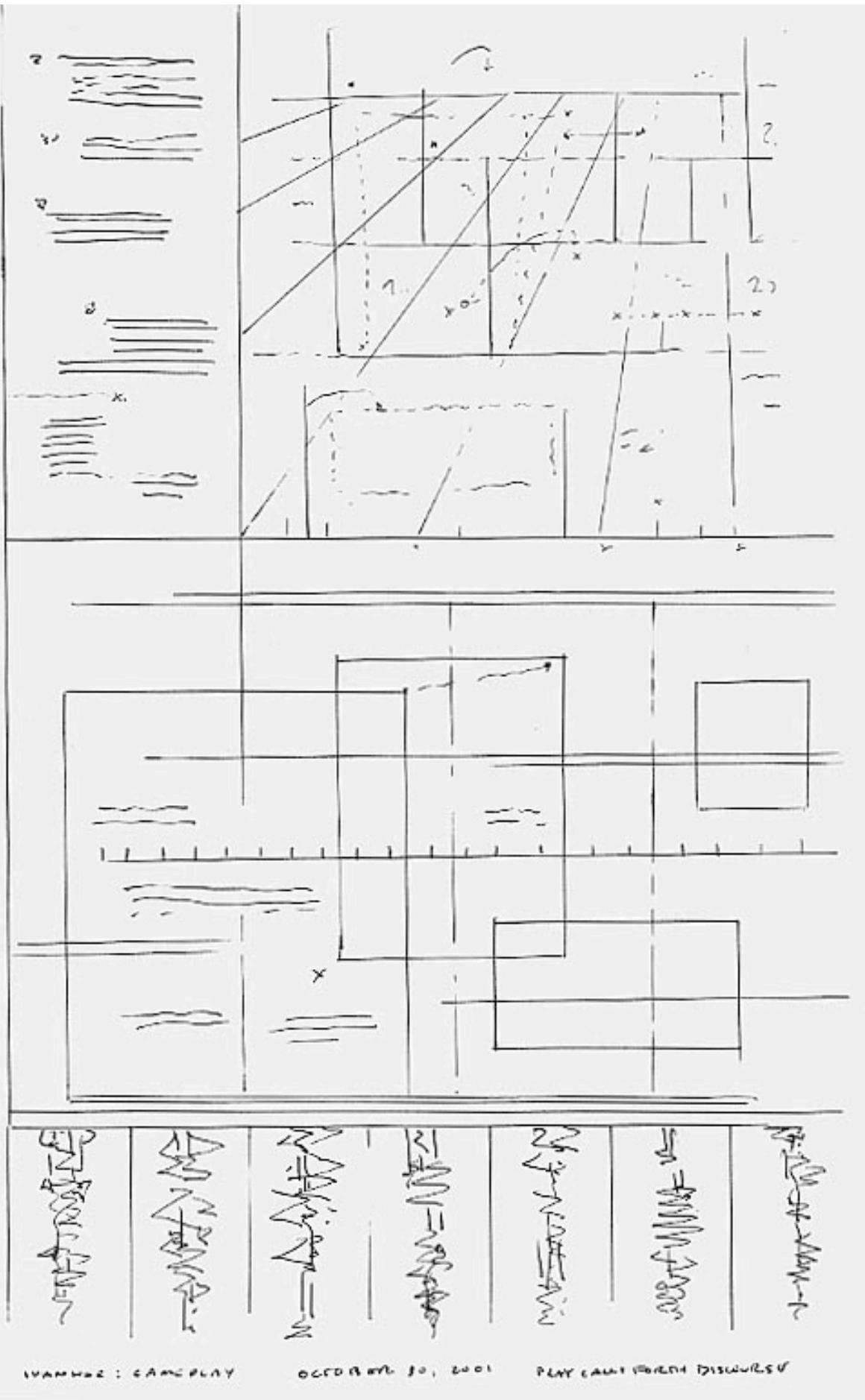


This has been too hard by half, but Espen A. defines ergodic literature against the primrose path: "nontrivial effort is required for the reader to traverse the text" (Aarseth 1). Half Rome wants to know, What's not ergodic?

Stop me if you've heard this one: "Are there circumstances (like those at play in the Ivanhoe Game) in which ‘effortful mental operation' to negotiate our conflicts is desirable precisely because it reveals perspectival and cognitive differences?” One’s Browning is a testament.

So the solution to imagine is much more than this

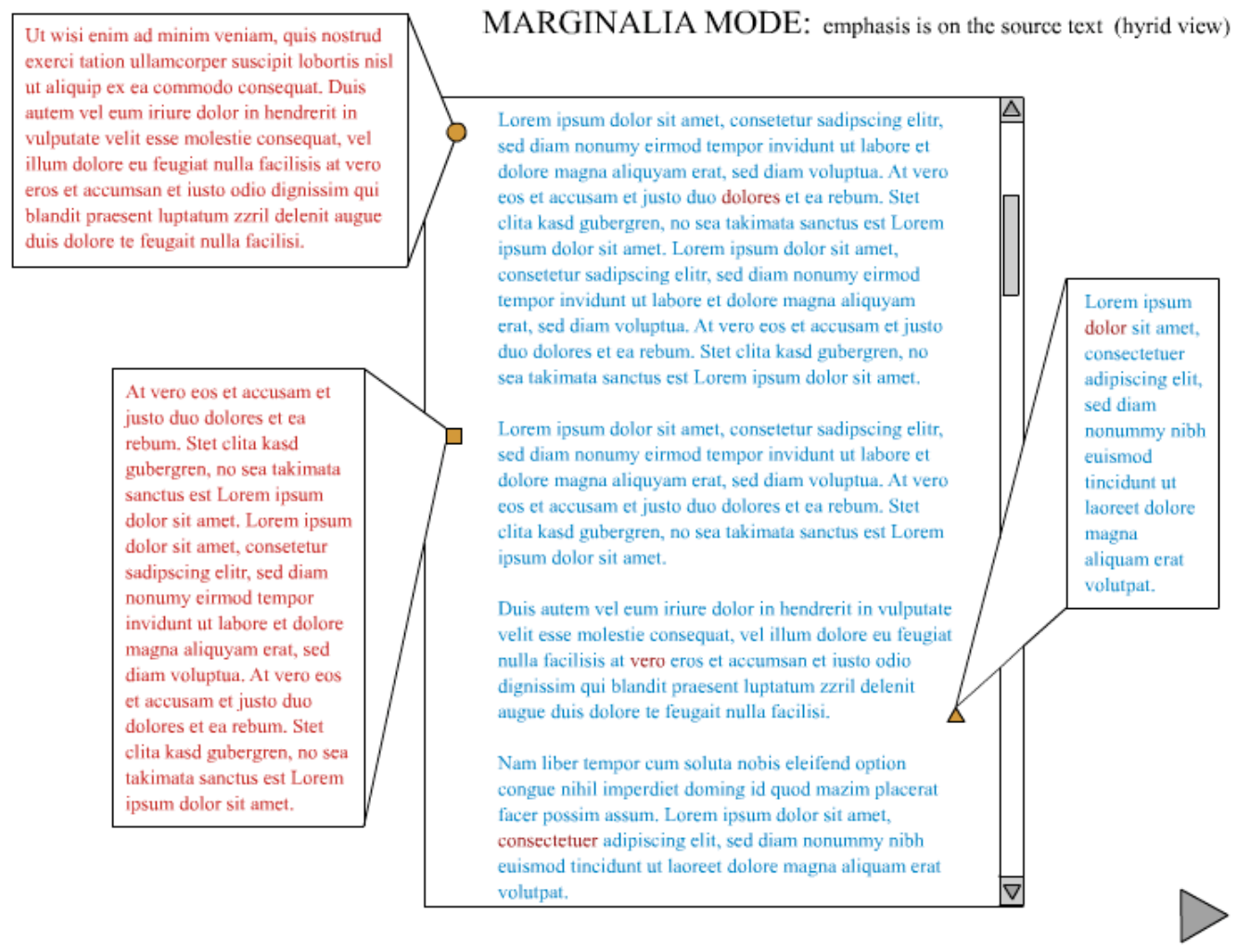


or that.

SPATIAL DISPLAY MODE:

Lorem ipsum dolor sit amet, consetetur sadipscing elitr, sed diam nonumy eirmod tempor invidunt ut labore of emphasis is on total game play

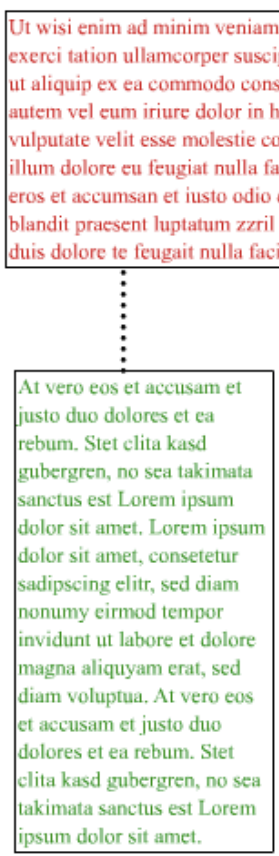
dolore magna aliquyam erat, sed diam voluptua. At ver eos et accusam et justo duo doloreswet ea rebum. Stet clita kasd gubergren, no sea takimata satnous est Lorem ipsum dolor sit amet. Lorem ipsum dolor sit arrexconsetetur sadipscing elitr, sed diam nonumy eirmod tempor invidunt ut labore et dolore magna aliquyam erat, sed diam voluptua. At vero eos et accusam et justo duo dolores et ea rebum. Stet clita kasd gubergren, no sea takimata sanctus est Lorem ipsum dolor sit amet.
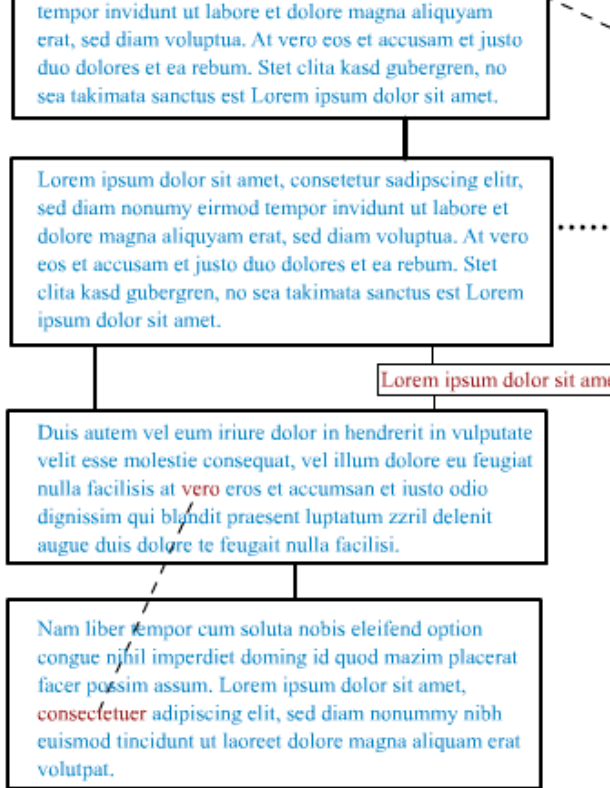
acilisi. 


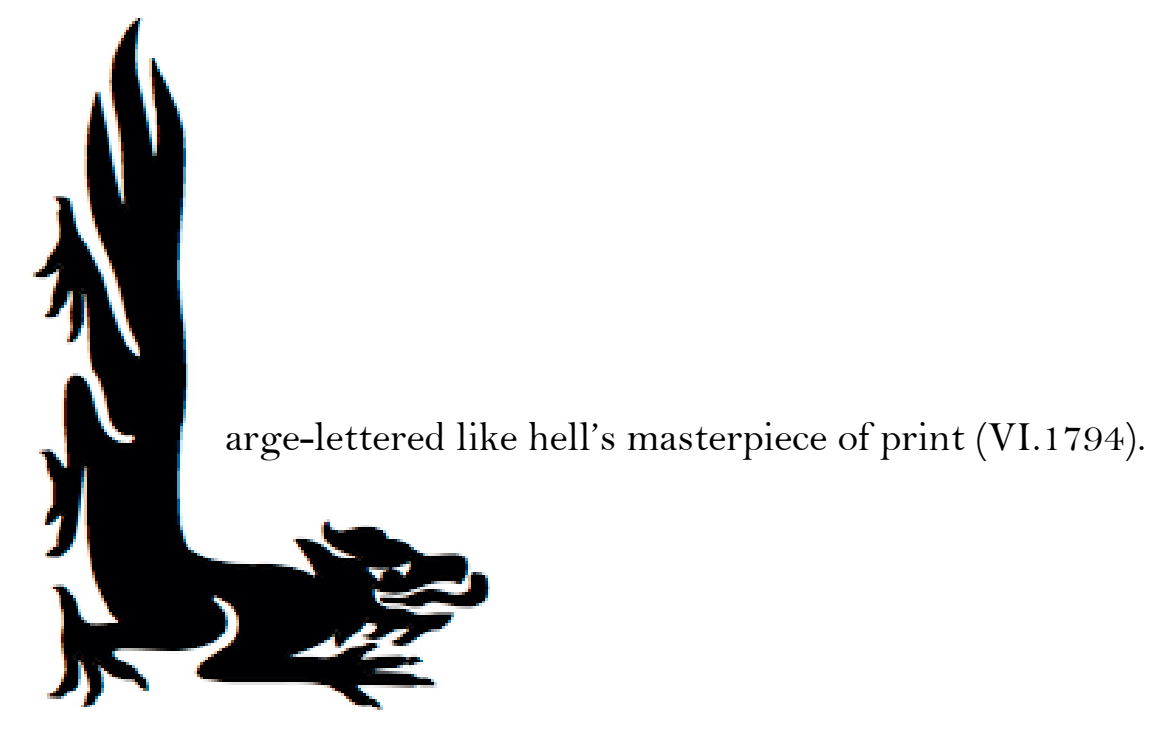




\section{Coda: Speculative Computing}

By now the term "speculative" has slipped into my writing in several different contexts: first when I cite Swift's satire of a Llullian combinatorial device busily cranking away in cloudy Laputa (a "Project for improving speculative Knowledge by practical and mechanical means"), and then in Ada Byron's early realization that algorithmic devices like Babbage’s Analytical Engine have subtle, extracurricular benefits:

For, in so distributing and combining the truths and the formulae of analysis, that they may become most easily and rapidly amenable to the mechanical combinations of the engine, the relations and the nature of many subjects in that science are necessarily thrown into new lights, and more profoundly investigated. This is a decidedly indirect, and a somewhat speculative, consequence of such an invention. (Lovelace, "Note G")

It returns later, when I describe and interrogate the notion of aesthetic provocation and speculate forward from the subjective and intersubjective premises of IVANHOE to its possible manifestation as Ivanhoe Game software. And of course every branching past or future expressed through our Temporal Modelling nowslider tool is a concretelyimagined, interpretive speculation.

Speculation is the first denizen of the curious realm of the "patacritical, that "science of 
exceptions" which seeks to expand our scope of thinking about ordinary and extraordinary problems through the proposal of "imaginary solutions," solutions which crack open the assumptions through which those very problems are framed. ${ }^{42}$ It is, perhaps, a strange bedfellow for the pragmatic interests that shaped my work on the projects outlined here — but then, pragmatism itself (as, in William James's terms, the “attitude of looking away from first things, principles, 'categories,' supposed necessities; and of looking towards last things, fruits, consequences, fates”) makes an odd counterpoint to the inward motions of subjective structuring that characterize this work and to its frequent backward glances in deriving and interrogating scholarly primitives (James).

Like 'patacriticsm, speculation is almost instantly reviled by some (certainly stalwart) souls who point out its negative connotations; speculators act without thinking through all possible outcomes, they take uncalculated risks for incalculable rewards, they are the teenagers of the academy, they are dot-bombers.

When I proposed the moniker "Speculative Computing Laboratory" — SpecLab — for an as-yet unnamed group coalescing at the University of Virginia at the turn of the 21 st century around a set of risky, wacky, brazen projects in digital humanities, I did so with full knowledge of these uses, and more — with a nod toward a highly technical application of the phrase. Computer scientists define "speculative computing" in terms familiar to us from Ada Byron's observations: 
Speculative computing is a technique to improve the execution time of certain applications by starting some computations before it is known that the computations are required. A speculative computation will eventually become mandatory or irrelevant. In the absence of side effects, irrelevant computations may be aborted. However, a computation which is irrelevant for the value it produces may still be relevant for the side effects it performs. (Osborne)

It is this particular technological burden on the notion of the speculative that most attracted me - how the preoccupations of projection, without regard for the cost of relevancy, could become active: embodied in real work, real artifacts, real happenings and doings, in a digital environment. The resolute notion, in Osborne's definition, of speculative efforts — our "imaginary solutions" — becoming either "mandatory" or perfectly "irrelevant" also appealed, and I must confess to enjoying a certain deformative reading of the paragraph: s/irrelevant/irreverent.

Of course, irreverence (like the ergodic, "non-trivial effort" that may characterize a reading of this dissertation) was never a real goal. My own career as a graduate student in humanities computing has been shaped by a necessary radiance, inherent in the projects about which I care most, outward from the archival impulses of IATH, through the hermeneutic designs of SpecLab, and into a fringe which is at once the center: ARP, a group for Applied Research in 'Patacriticism, which labors steadily, at this writing, to embody elegant speculations about humanities scholarship in practical tools and 
working institutional structures for what comes next.

In a review of Jerome McGann's Radiant Textuality, Johanna Drucker (a key instigator of speculation in digital humanities $)^{43}$ contextualizes our goals in terms of inevitabilities in the outlook for aesthetic theory and criticism:

The world is changing. Just as dramatically and radically as it did under the influence of post-structuralism and deconstruction. But the changes being wrought are taking place in day to day activities that are, for the most part, far from the seminar rooms that spawned theoretical activity in the past. These changes are being enacted and performed in the making of electronic instruments whose premises will change the way humanities is done... Making things as a way of doing theoretical work pushes the horizons of one's understanding "because poiesis-as-theory makes possible the imagination of what you don't know.” (Drucker, “Theory as Praxis”)

Enactment and performance are watchwords of the interpretive environments described here. I'm always conscious as a designer, and as a collaborator in a design community, that the instruments and environments I help bring into being embody a doubled relationship with the notion of poiesis-as-theory. Drucker and McGann discover the first strand of that helix: that we practitioners, we tool-builders, open ourselves to a fresh kind of critical engagement with the materials and ideas and histories of arts and letters. I write "fresh" rather than "new," because, as both these theorists have shown, 
our work has a rich ancestry in critical and creative lines that, at times, have seemed to die out or be subsumed into other cultural and intellectual lineages. In my own experience, iterative development of software and systems — the making and refining of things that go (“I go, I go, look how I go!") — teaches reams and realms more than mere abstract interrogative thought. The fringe benefits of constructive and pragmatic work are sometimes so great as to be a monumental distraction; this is the reason we often joke that the making of the Ivanhoe Game is the whole of the game.

The second, closely-wound strand of the poiesis helix involves both the practical and rhetorical goals of our undertaking: we wish to share the wealth, by making it possible for the users of SpecLab toolsets and environments to participate in the same fresh engagement with theory that making things makes possible. Every one of the interpretive environments I've described asks its inhabitants ("users" is sometimes too pale a term) to produce digital artifacts or perform mediated actions and manipulations in a representational landscape. The degree of abstraction of those representations varies, but they all embed a pragmatic understanding of critical and hermeneutic operation. For each concept, they supply a set diagrammatic or algorithmic or combinatorial tools for interpretive making and doing. Of each product of those instruments — whether a model or a performance or a set of embodied relations - they ask its maker one instrumental question: does it work? (Does it parse? Does it go?)

This is, perhaps, the ultimate brand of speculative computing — a close kin to the risky 
speculation we teachers and mothers engage in as we invest our energies in pedagogy, or in the bringing up of babies. We make, and make plain, worlds of opportunity with only the slightest notion of what their new inabitants will make of them, and in them. But we frame those worlds as active, and populate them with objects and agents and ideas, and we makers watch and learn. 


\section{Appendix A. “Temporal Modelling: Conceptualization and Visualization of Temporal Relations for Humanities Scholarship” by Johanna Drucker and Bethany Nowviskie}

(Draft Article submitted to Information Design Journal, 29 November 2001; reproduced here with permission of Johanna Drucker)

\section{A. Overview and premises}

Self-conscious awareness of time as an aspect of the human experience is an age-old aspect of culture. Despite wide cultural and historical differences, a surprisingly unified array of conventions has arisen for the representation of time and of temporal relations. These conventions for recording the shape and passage of time and the ordering of events in temporal sequence are cross-disciplinary. However, their grounding in areas of inquiry based in empirical research, particularly the natural and social sciences, has meant that they are premised on common assumptions of time as uni-directional, neutral, and homogenous.

From the outset of our Temporal Modelling Project, we have asserted that certain counter-assumptions are essential for the modelling of temporal relations within humanities scholarship, extending, rather than contradicting existing conventions.44 
Documents specific to the humanities are often fraught with complexities and contradictions regarding the ordering of elements in a temporal scheme, and their interpretation relies upon acknowledgement of subjective perspectives. The simple fact that any human-authored document represents an individual and inherently fragmentary point of view from within events, rather than an objective record from a presumed external stance, necessarily suggests that our counter-assumptions are integral to humanistic inquiry.

Our challenge is to create a framework for graphically representing such elusive and subjective seeming phenomena. What notation scheme allows us to map anticipation or regret, both ways of conceiving of future and past in modes that inherently involve transformation of past record and future events? Consider the unfolding of multiple narratives simultaneously with contradictory accounts - another standard feature of historical record - or of individual memory against the backdrop of official history. How may we create a graphical communication scheme that allows these concepts clear enough representation to be useful? What metaphors and templates are capable of presenting a conceptualization framework within which interpretation of such events may take place according to these mutable and inflected timescales in a way that may nonetheless prove useful as a research tool for interpretation, analysis, and display of temporal data? How may we diagrammatically represent the inner standing-point as a concept within temporality? 
The conceptual premise of our project is framed, therefore, within these assumptions:

time may be experienced as a uni-directional flow within human perception, but the interpretive ordering of events has forward-branching (prospective) and backward-branching (retrospective) options; time is inflected by emotions, mood, atmosphere, rather than being neutral; the shape of time intervals (granularity, scale, and metric) varies according to subjective perception. It is heterogeneous, not homogeneous.

The technical problem we set ourselves as a result of these premises was to create an interactive tool-set for representing and modelling temporal relations in humanities data, in advance of the content modelling necessary for development of a database, DTD, or XML mark-up scheme. Our assumption is that a tool-set for visualization of temporal relations would provide a useful framework for interpretation of documents, accounts, narratives - the complex and fragmentary information typical of human records. Designed with sufficient constraints, this tool-set could be used to give rise to a formal knowledge representation scheme. Though the established model of humanities computing has become accustomed to the development of content models first, with display of information following as a second phase (with all awareness of the interpretive nature of display), recent developments in visual computing have begun to demonstrate the promise of composition spaces, such as the one we are proposing, as primary sites for input and modelling. ${ }^{45}$ 
Our goal is to devise a set of interactive tools for visualizing subjective, inflected, nonhomogeneous temporal relations. The composition space we are creating will effectively model temporal relations within the formal constraints of schema development. To inform our project, we followed the following research agenda. First we set about reading through a considerable variety of works from different disciplines that describe models of temporality. These included humanities fields (philosophy, narratology, structuralist discourse analysis, history, knowledge representation), social sciences (particularly anthropology and religious studies), informatics (formal logic, linguistic analysis, temporal database development), the natural sciences (biology, geology, physics and relativity theory), and visual design (art history as well as graphic methods for information design). In our next phase of research, the focus will be on topological mathematics and the spatial modelling of events, the analysis of temporal elements in narrative and linguistics (including deixis and tense modalities), and the field of diagrammatic reasoning and semantics. These three areas - more specific than the broad range we investigated at the outset - describe the intersection of our concerns to call out content from documents, register the complexity of temporal events, and exploit formally constrained systems of visual representation within the composition space.

Because we are humanities scholars with digital skills, rather than computer scientists or information architects primarily, we worked from our literature review towards the 
distillation of a set of conceptual primitives for the representation and modelling of elements in temporal relations. We have followed a few key guiding principles: to adopt established terminology where possible, to keep our system as visually and conceptually simple as possible, to be as general (rather than idiosyncratic) as possible in the creation of our interactive tool set, and to keep in mind that we want a system that allows for subjective experience of temporality to be graphed with formally constrained tools that are compatible with computational methods.

The reason for this final point is that merely "picturing" temporal relations, though extremely useful for pedagogic or illustrational purposes within interpretive activity, doesn't provide the desired technical outcome - a formal expression of the user's interpretive stance toward his or her data, a content model which could be used to mark that data in XML or place it in a thoughtfully designed database.

\section{B. Summary from the literature review}

The literature on time and temporality cuts across humanities, social sciences, natural sciences, and informatics. However, a surprisingly concise set of terms and basic concepts emerges from examining this range of disciplines. Since mapping so large a terrain in detail would be an ambitious undertaking, our discussion is intended only as a schematic overview. We will focus on crucial concepts within three areas. The first, philosophy, is concerned with issues of ontology and metaphysics in the conception of 
time and temporality in ways that underpin assumptions for many fields of human inquiry. The second, logic, is concerned with formal systems for determining time and temporality for fields such as informatics and their requirements for instrumental and practical applications. The third, discourse analysis, is concerned with the thematic description and material encoding of concepts of time and temporality in natural language. With the exception of 20th century developments in relativity, the concepts which emerge in these three areas were understood and established by early centuries of the Common Era. ${ }^{46}$ More specialized terminology and more elaborate scholarly schemes of analysis have emerged in recent decades, but the fundamental conceptual underpinnings have remained remarkably consistent across historical periods and intellectual fields of inquiry.

In almost every discipline, an important distinction can be made between absolute and relational time. Absolute time is an a priori given, conceived as a structural container of events, while relational time emphasizes temporality as a product of the relative sequence of events within a frame of reference where order and duration create the temporal structure. These distinctions, however, are not always clearly made since the assumptions on which they operate are often inherent in a disciplinary perspective. In particular, the idea that time pre-exists events has a strong foothold in the natural sciences, where the ontological existence of time goes largely unquestioned. Even the most intuitive interpretations of the subjective experience of temporality are often framed in relation to this a priori concept and the empirical premises it reinforces. To 
shift this ground we have to jettison the idea that time or temporality in themselves are being modelled in our system. Since our goal is to create an interface for interpretation of temporal relations in humanities data, we will want to be aware of these assumptions but focus on the ways temporality is understood thematically and is encoded in representations such as language and other symbolic forms.

\section{Philosophy: Ontology and Metaphysics}

To begin, we will situate our inquiry within what Fabio Schreiber terms the study of temporal ontologies or "the major issues in the nature and structure of time."47 The empiricist bias is evident in the simple assumptions that one may describe "the nature" or structure of time as a unified, homogenous singular entity. Working within the field of informatics, Schreiber is motivated by a pragmatic desire to establish the parameters for temporal considerations such as the synchronization of distributed computational systems, but he begins by surveying literature at the intersection of philosophy, history, and informatics. Schreiber provides a useful list of descriptive approaches to understanding what time "is" in its inherent form. A fairly comprehensive set of intuitive insights in clarified form can be culled from his literature review. ${ }^{48}$ These begin with a distinction between linear and circular conceptions of time. The linear conception reinforces the idea of the uni-directional flow of time's arrow, while the circular suggests the repetition of life cycles, of circadian rhythms and patterns, or of other apparently identical replications of temporal sequences. Ontological understandings of time are conflated with representations in that opposition, since there 
is almost no intellectual support for the idea that absolute time has a circular or cyclic form. ${ }^{49}$ The other basic concepts Schreiber lists are more self-evident: the contrast between a belief in infinity and the human experience of the finiteness of time; an experience of discrete moments or units of time as against its perceived continuity and flow; an absolute sense of time described as past, present, and future; and a relative sense of time described in terms like before, after, or concurrent.

Schreiber makes clear that the flow of time is considered an objective feature of the physical world in much of western thought, providing scientists with philosophically supported assumptions. This flow can be understood in the language of formal logic and linguistics, in order relations, or charted against various metrics. Even the idea that temporal measures are arbitrary (hours, minutes, seconds) reinforces rather than undercuts the conviction that time "itself" exists as a container for events. Conventions for measuring time, marking its divisions and subdivisions according to named intervals, follow calendrical, horological, and other extrinsic time-keeping systems, each of which is bound to historical and cultural realms. Thus extrinsic reference frames may be sidereal, physical, biological, time-stamped and dated, or cultural. Religious and sacred times overlay and interpenetrate secular calendars even when the same system of dates is used as a scaffolding for both. Anthropological research offers evidence of temporal schemes that mark complex multi-phase systems in parallel to each other, but no matter how many different patterns are marked out, the system's premises don't challenge the a priori existence of time or its unidirectional flow. 
The wide-ranging scholarship of J.T. Fraser, who made the study of time his life's work, provides another useful framework. ${ }^{50}$ Rather than providing descriptions of temporal ontologies, Fraser's prolific scholarship systematically examines the ways time has been understood from various disciplinary perspectives. His list of descriptive rubrics includes:

eotemporality: the rational progression of temporal events in an apparently sequential form;

nootemporality: time as experienced by the human mind;

psychotemporality: perceived time, psychologically inflected;

sociotemporality: time proper to a specific social system or condition;

biotemporality: temporal distinctions operating with a continuous, organic present (with apparently cyclic and other purely linear patterns);

atemporality: the temporality of physics in which the universe is simultaneous, unordered, chaotic;

prototemporality: undirected, discontinuous, primary.

In Fraser's discussion, these concepts assume time as an a priori condition, available to description either as a sequence of events in human experience or as events that may be ordered within a descriptive schema. Even such a subjectively-oriented concept as psycho-temporality is defined against the idea that its subjectivities be measured and marked against a normative, a priori extrinsic temporality. Fraser describes these subjective systems of perception as a contrast with "time" as an absolute. Fraser's 
approach disregards the linguistic, visual, or symbolic systems in which concepts of time are encoded through their representation, taking the concepts and systems as descriptions of time itself, rather than as intellectual constructs to be analyzed. Therefore, we would suggest a key addition to Fraser's approach corresponding to the final category of this survey (see below, final section): discursive temporality: the representation of time in discourse. We would further suggest modifying Fraser's discussion with a clear distinction between the assumption of an objective perspective (in either metrics for charting time or the assumption of time as an a priori given) and the recognition of subjective experience within temporal dimensions as a point of departure for their apprehension and representation. By distinguishing the intellectual representation of concepts of time and temporality from a conviction of the a priori ontological existence of time as something in itself, we are establishing the foundation of our work on a self-conscious attention to representation, rather than a presumption of external realities and their absolute form of existence.

Fraser provides a useful panorama of approaches to the characterization of temporal schemes to suit the requirements of individual disciplines. The convention of the timeline is the overwhelmingly familiar representation of this idea, with its apparent ordering of empirically observed data against an extrinsic metric. Whether used as a simply linear ordering device, or as a means of organizing documents, evidence, or measured data, or as a method of record keeping, time lines can be used to represent 
simple and complex relations of information. In graphic and visual terms (see discussion below), the linear form of this temporal axis has proved serviceable for temporal record keeping in fields as diverse as informatics, biological sciences, medical observation, economic and meteorological graphs, and other fields in which data has temporal extension or duration.

\section{Logic}

In formal logic and informatics a precise terminology describing the relations of intervals and events with a linear system emphasizes the relational or relative ordering of temporal events. This work is distinct from that which we are terming "discursive" temporality in one significant respect: the formal logicians, exemplified in the muchcited work of James Allen, are "de-tensers" who enunciate a vocabulary for describing relations among time intervals rather than focusing on the language in which temporal experience is marked and represented. ${ }^{51}$ In Allen's much-cited essay, "Time and Time Again," relational diagrams lay out a logical framework for all possibilities of order and sequence of events -- if such events are taken to exist outside of their representation. These temporal logics cannot always be correlated to an absolute or extrinsic dating system (calendar or clock time) and they may be adequately constrained by an intrinsic dating system as pseudo-dates. In either case, the formality of Allen's logical system allows for a fully dis-ambiguated description of the temporal relations while accommodating forward branching options, a desirable feature for computational situations in which a single, determinate past might have a multiplicity of future 
options. Allen's logical relations include these terms (and their complements):

before

overlaps

during

starts

meets

finishes

equals

Each is capable of distinct visualization as sets of arrows whose formal relation precisely matches the temporal relation and corresponds to its verbal description. For Allen, the concept of tense is cast entirely within formal language, which makes it compatible with the requirements of informatics. The concepts of temporality needed for time-stamped database operations make use of similarly formal logic in making the distinctions between moments at which a fact is stored in a database, moment of a query, or moment at which a fact might be true within a modeled reality. These systems depend upon internal clock mechanisms, intrinsic systems of highly formal, unambiguous temporal relations. They therefore lend themselves to formal description rather than either correlation with extrinsic systems or subjectively inflected and ambiguous tense modalities. $^{52}$

\section{Discourse analysis}

In contrast to the formal approach provided by logic, the work of linguists and scholars 
of language in literature and narrative meets other challenges in assessing the representation of time in the tense modalities that are the foundation of fictional, historical, or other documentary narratives. This approach focuses on the encoding of assumptions about temporality in symbolic representation in natural language whether in an utterance, document, or narrative. The first problem is that of identifying the linguistic markers of tense or other temporal feature. The next resides in understanding the cultural, psychological, or other symbolic value by which the temporal system is inflected.

In "The Productions of Time, " Mark Steedman provides an extensive study of tense modalities or tense logic in language incorporating the classic work of A.Prior in discussion of speech, reference, and event points within linguistic representation, as well as a summary of contemporary work in this area. ${ }^{53}$ Rather than attempt a description of events grounded in formal relations of intervals, Steedman and his colleagues seek to elucidate the semantic implications of distinctions embedded in linguistic terms. Achievements, measured at or in a particular moment are contrasted, for instance, with accomplishments, which are extended in time, and activities, which endure for a set period. These descriptive categories clarify the means by which natural language encodes cognitive concepts about time and temporal relations.

In narrative theory, the creation of constraint logic programming as an analytic and interpretive tool allows narrative elements to be defined within a system of internal 
references for temporal relations. In such a system, each element is analyzed and its temporal identity constrained within a formal system in order to extract an ordered sequence of referenced events out of the language of experience, action, or descrption in the narrative. ${ }^{54}$ These approaches are dependent upon the careful analysis of tense indicators in syntax and discourse structure. Pamela Jordan, a linguist studying narrative uses of tense markers, addresses the distinctions among narrative reference frames. ${ }^{55}$ Tense markers such as "here" and "now" not only describe relative time frames, but also link the representation of time to individual subjectivity. The concept of deixis, derived from structural linguistics and applied to narrative theory, refers to the way subjectivity (individual speaker identity and position) is structured in language. Though classical narrative, as defined by Aristotle's unities of character, action, and location, assumes that time and space are universal, continuous, and coherent, such assumptions are not part of all narrative frameworks. Self-conscious manipulation of these unities is part of 20th century literature and its theoretical and interpretive approaches -- which also extend to the analysis of linguistic documents in historical studies.

Historians and anthropologists note that ideological and cultural values often inflect time systems. Herbert Bronstein, in "Time Schemes, Order, and Chaos: Periodization and Ideology," points to the repetitive cyclic conceptions inherent in a notion of eternal being and radically contrasting ways this concept operates within Jewish and Christian approaches to historical chronology ${ }^{56}$ The difference between an anticipated or already 
acknowledged appearance of the Messiah serves as an organizing feature of all historical events, casting a markedly non-neutral interpretive frame on the description of human experience. Any such historical scheme embodies a world-view laden with a sense of progress towards or away from a culturally sanctioned goal of progress, salvation, enlightenment, rebirth and other defining idea. The very division of history into discrete epochs or periods, such as ancient, medieval, or modern, marks assumptions about shifts in cultural paradigms along an irreversible temporal axis.

Cross-cultural perspectives demonstrate the bias inherent in concepts of temporality that are taken to be intuitive or to organize social relations into a network of cultural activities. Most conspicuously evident in the use of various time-keeping schemes, these differences extend to notions of dream-time, ideas of floating points of the present within a non-linear concept of past and future, and other alternatives to the rational system of logical, uni-directional order in western time concepts. ${ }^{57}$

A final contribution to the study of tense and alternatives to linear, uni-directional timearrow frameworks comes from the study of relativity and its influence across a wide spectrum of cultural activities. ${ }^{58}$ Fiction and narrative, as well as scientific discussions of event modelling, historical patterns, and events within the realms of physics, all lend themselves to description according to models derived from what is termed spacetime. ${ }^{59}$ Scientific debates about the absolute existence of a time arrow focus on the second law of thermo-dynamics (the tendency of chaos to increase in the physical 
universe along an apparently asymmetrical temporal axis). But in narrative imagination, the theory of relativity provides suggestive starting points for the reordering of perception. ${ }^{60}$ The multiple temporalities available in such systems fragment the unity of time as well as its illusory order in human experience.

As a summary of this brief survey, then, we can see that the apparent order of time as a given, a priori container for experience has a counterpoint in the conception of temporality as created and shaped by the ordering of events, objects, elements, and effects in increasing layers of complexity. Fundamental differences are also marked between objective and subjective conceptions of temporality, and among variously inflected interpretations of the value of events within temporal orderings. From this literature review, we elaborated a stable nomenclature of concepts. These in turn informed our elaboration of "temporal primitives" that comprise the elements of our conceptual scheme. As a parallel to this literature review, we conducted a survey of visual conventions for the representation of time and temporally marked information.

\section{Graphic forms for representing time and temporality}

In assembling a visual archive on which the Temporal Modelling Project could draw, we became conscious that a limited set of graphic conventions has been deemed effective by Western cultures for picturing data in time. These conventions are made meaningful by shared assumptions that time is uni-directional, neutral, and homogenous.

Two fundamental elements of any temporal diagram are the reference frame through 
which it is structured and the notational vocabulary with which temporal relations are expressed. Reference frames make the expression of temporal relations possible by defining the rules under which the visual system operates. These frames are either extrinsic to the data (assuming an objective time framework against which the absolute temporal position of an element can be measured) or intrinsic to it (based solely on the relations among the elements themselves but traditionally assuming a linear chronology). In some instances, reference frames present a combination of intrinsic and extrinsic measures (e.g. the perceived time of an experience and the actual time of the event measured against a standard time-keeping device). Conventional temporal notation vocabularies contain three types of markers: for points or discrete instants in time; for intervals or segments of time; and for events, which are occurrences in time. These primary notations are expanded and refined by text labels and shape- or colorcoding.

Diagrammatic representations of temporal relations fall into three basic categories: linear, planar, and spatial. Linear diagrams, or timelines, are by far the most simple and prevalent forms. Traditionally, they consist of a single axis on which is laid a stable metric and a sequence of markers or labels representing the linear progression of events in time. The timeline becomes in effect a linear spectrum from early to late, predicated on a homogenous granularity and through which data can exhibit only three relative temporal conditions: earlier than, later than, or (sometimes awkwardly) simultaneous with. (See figure 1.) 
Planar diagrams chart temporal relations on two axes. Sometimes, as in calendar grids, which mark days against the larger structure of weeks, both of these axes are temporal. Often, however, time is marked according to a uniform metric along a single axis and data is charted both temporally and in some other quantitative way against the other axis. These diagrams can take the form of the familiar bar or line graph and may present information about a single data type (bivariate graphs) or about multiple information streams (multivariate graphs). In most cases, this form does not emphasize temporal relations, but rather the evolution of a specified attribute of the charted data over time. Like linear diagrams, the planar form presents the flow of time as unidirectional and asymmetric. (See figure 2.)

A spatial diagram, the least common representational scheme, attempts to map data on multiple axes - sometimes literally tracking events as they move in time and through geographical space, and sometimes modelling data in a three-dimensional format in which none of the coordinates measure literal space. In print media, these diagrams often take ingenious forms in their attempt to overcome the limitations of the page. Digital spaces offer new opportunities for three-dimensional diagrams. (See figure 3.) The visual conventions used for schematically representing data in temporal relation generally share the assumptions we have cited above: time is unidirectional, neutral, and homogenous. The design we propose challenges all three of these assumptions and creates a visual scheme in which alternative approaches may be represented for purposes 
of basic research and visual display. Clearly, these assumptions have produced adequate ways of interpreting or presenting interpretations of time, but we suggest that they are not the only way to model temporal relations and that they have no greater validity or objectivity than our system for representing subjective temporality. Humanities scholars deal with many variables in the temporal relations expressed in documents and accounts and have need for a less rigidly empirical and more flexible system of representing these relations.

\section{Contribution of our project in conceptual terms}

The first assumption on which conventional temporal representations rest is often referred to as the time arrow, a familiar notion that time has a unidirectional, irreversible flow in which the past is unchangeable, the future unknowable, and the present elusive but all-present. The very concept of the time-arrow is itself premised on a second assumption - that time is unified, a pre-existing whole that simply is. According to this notion, events are contained in time in much the same manner as objects are contained in space. Finally, the conventional conception of time used for measuring phenomena in objective, empirically-based time measures, is that time has a single metric. Though degrees of granularity may differ in their usefulness for assessing various phenomena or different parts of complex events, the underlying assumption is that there is a single unified temporality within which such granular differences can be reconciled (as different scales of the same measure). 
We suggest that all three of these are assumptions, and that the vocabulary of interpretive possibilities may be extended through addition of alternatives that modify each of these assumptions.

The conventional idea that time is an arrow, and that events follow each other in fixed sequence such that past events are unchanged by those that succeed is the first assumption that is contradicted by the way temporal relations are experienced within individual human perception and within the broader context of documents that form the basis of humanities research. The frames of interpretation common within history or narrative, for instance, show that a map of past events may change dramatically according to new information, or occurrences, that do not merely recast our interpretation of events but alter our conviction about what actually occurred. (The development of theories of the geological history of the earth in the 19th century offer a very clear example of such a transformation, since biblical measures of past events, taken quite seriously as metrics by historians as well as theologians, were subject to radical reconfiguration in order to conform to empirical evidence offered in the physical record.) Similarly, anticipation of future events and the degree to which this anticipation shapes the present, a major aspect of narrative practice in prose and drama, is difficult to chart on a standard time-line.

The idea that time is neutral and that it provides a bland, container-like setting for events outside of their individual existence is countermanded by the realization that 
temporality is constructed precisely by the relations among elements. Tensions and pressures exerted by such events inflect all temporality with subjective qualities. The idea of "the distant future" or "someday" or "after my lover comes back" - all quite logically compatible with subjective experience of temporality - are not able to be absorbed into a neutral concept of time with a stable metric that exists independent of events. The relations among events separated by time, rather than an experience of time itself, is the focus of such experience.

This leads directly to our final alternative to the idea of time as a single, linear, scale of temporality that is homogenous and consistent. In much humanities-based research and in lived human experience, subjective notions of time are distinctly different depending upon circumstances and emotional or other investments. Not only is the perception of granularity different among various areas of particular events or phenomena, but the relation of parts to each other, parts to a whole, or metric scales to each other are not always able to be unified within a single homogenous frame. (Clearly the appropriate granularity for a historian documenting the burning of Atlanta during the Civil War, for instance, is quite different from that used in the narrative of Gone with the Wind in either its film or book versions). Breaks, ruptures, inequities and discrepancies in pacing are all elements of lived experience of time and its record in humanistic documents. These ruptures or lacunae are often the periods of greatest interest to the humanities scholar and lay user of time-based digital media alike. 
With this conceptual framework in place, our work became focused on the elaboration of an effective visual design for the interactive toolkit and composition space.

\section{E. Contribution of our project in technical terms}

\section{Conceptual primitives}

As a first step in designing an interactive graphical system for temporal modelling, we established a working list of conceptual primitives, the least common denominators of our model. This classification exercise was meant to contribute to the later development of the formal schema for subjective temporality that would define and constrain our notational system. It resulted in a table covering the range of objects, relations, and actions we hoped to combine in a software prototype. (See Figure 4.) Objects are the basic graphic elements of the composition and display spaces, such as timelines, points, intervals, granularity markers, and events. Objects are by definition manipulable, and users may manipulate them both by position (placement within an established line, grid, or other metric frame) and through the application of subjective inflections (a set of graphical markers to indicate values from a pre-established set or in a user-defined mode). Relations are the fundamental structures that may be expressed using our system, such as order, rupture, certainty of temporal position, and boundedness of events or intervals. Actions encompass all the operations on objects and relations that we want to enable within the software prototype. Positioning and labeling elements, ordering and re-ordering them, attaching and detaching metrics, and 
inflecting temporal relations are all examples of defined actions in our model of conceptual primitives.

\section{Special interface features}

We have developed three special interface features to enable important actions or operations humanities scholars may wish to perform on their data. Each of these facilitates display and interpretation of user-created diagrams in the composition space and highlights the utility of our prototype for thoughtful analysis and content modelling. The first feature involves draggable, transparent layers on which whole systems may be placed for interactive overlay comparison. We have discovered that layering - when coupled with a carefully designed color palette for user-labelled points, intervals, and events - is a simple but effective method for pattern matching in composition space sketches. It is possible to intensify color on overlay for those graphic elements that are found to repeat and blend color to alert the user to repetitions with a difference. Best of all, we can permit completely unrestricted dragging of the layers so that users can compare intervals on two or more timelines which may be far separated by time's arrow but exhibit similarities in the pattern of objects they contain. A second interface feature also emphasizes patterns in temporal data. We are experimenting with the use of embedded dial interfaces to reveal and compare cyclical patterns or factors in the temporal compositions users may devise. By wrapping into concentric circles what may, in an initial model, have been figured as linear progressions of events, and by interactively turning and adjusting those embedded dials, our users 
will be able to experiment with cycles and repetitions in their own data. A possible added feature to the software would enable the mapping of linearly-conceived and interpreted user data against extrinsic cycles stored in the system, such as phases of the moon, tides, harvest almanacs, holiday cycles, or train tables.

The final special interface feature under development is the nowslider, an interactive editing system and display device which demonstrates that the inner standing-point is a critical element of any model of temporal relations, that it is figurable in diagrammatic terms, and that graphic presentation of the "now" facilitates the interpretation and display of human information. The nowslider is a control mechanism which firmly fixes a locus of interpretation within the progression of events of a narrative or history. By sliding the mechanism forward or backward "in time," the user can display alternate iterations of the future and past from the perspective of any given moment. These iterations can be figured as one person or entity's reinterpretation of past events based on new information, or as that person's changing expectations of the future in the light of the present. In this way, the nowslider paints reconsideration and retrospect as a dynamic force shaping history, and demonstrates that the future is as much an object of interpretation as the past. By editing with and for the nowslider, users may categorize the same events as both planned and unplanned, foreseen and unforeseen, or may inflect a single object or system on a timeline in a variety of different ways, from different temporal points of view. The nowslider concept supports our counter-assumption to the time-as-arrow convention: prospective and retrospective branching is a central 
feature of experienced temporality.

\section{Subjective inflections}

The visual and programmatic inflection of temporal relations is a fundamental component of our model, meant to reflect our second and third counter-assumptions: that time is not neutral and homogenous, but rather inflected and altered according to subjective perception. By enabling the display and analysis of temporal subjectivity we hope to engage humanists and facilitate their own research. Inflections are graphic modifications to the basic objects and relations of the display and composition spaces. We subjected our thinking on modes of temporal inflection to the same process of classification that resulted in our list of conceptual primitives, and produced a chart of inflections divided into two fundamental types: vocabulary or semantic inflections and grammatical or syntactic inflections. (See Figure 5.)

Semantic inflections are defined as those graphic modifications necessary for demonstrating temporal subjectivity, but which are not at all positional, not able to be dealt with through actions like layering and nowsliding, and are applied to objects requiring more than a text label for differentiation and evocative display. A semantic inflection is applied to a single graphic element on the screen and does not require the presence of other inflected elements to have meaning. Syntactic inflections, on the other hand, serve more than a labeling function. Like semantic inflections, they are not purely positional and are not able to be expressed through actions like layering and 
nowsliding. These inflections are grammatical in the sense that they deal wholly with temporal relations between and among elements, and therefore require a small system of inflected elements in order to be comprehensible. An example of a semantic inflection would be the color-coding of an event on a timeline to indicate its userdefined type or source, or the surrounding of that event with a graphical symbol or wash to indicate that it was emotionally "colored" by a certain mood or atmosphere. A syntactic inflection would, for example, alter the appearance of the timeline's metric to indicate a subjective sense of change in granularity. Time might be pictured as passing more quickly or more slowly depending on the user's adjustment of granularity tics. Event foreshadowing, a common element in literature and interpretation, is a syntactic inflection involving at least two objects in temporal relation: a precursor or Biblical type and the event it prefigures. Our visual notation set provides adjustable graphic systems for foreshadowing, causality, anticipation and dread, weighted and delayed impact, and other syntactic inflections useful for modelling humanities data.

\section{Challenges and Opportunities}

We have experienced the design of our visual notation system and the adaptation of conventional time-representation modes to our prototype as a series of challenges and opportunities, many of which we imagine are common to most information visualization projects which aim to establish new models of representation. Problems in building a graphic vocabulary centered around the balance between aesthetics and diagram semantics. For example, our early symbols for point objects - meant to represent 
isolated, brief moments in time - took up far too much temporal "space" on a timeline, despite being of a proper size for manipulation and display. The limited amount of screen real estate in our composition space prototype makes problems of clarity and clutter loom large, and we are constantly working to produce elegant, readable, and yet semantically dense visualizations. We are also working with a group of beta testers to refine our notational vocabulary and ensure that we produce symbols with clear and intuitive functions. The subtle implications of certain shapes, colors, and interactions only become clear when they are tested by a variety of people in modelling different types of data to different ends. An object of consideration at present is our system for indicating temporal uncertainty and overlap by positioning elements above and below the "line" of a conventional timeline. To some degree, such arbitrary systems can be made clear by labeling and become a matter of habit for the user, but the complexity of our model means that we must also consider the impact of subjective inflections on any established pattern of use. For instance, one proposed inflection alters the shape of the line itself, placing some events in troughs and others at peaks. How might this affect a user's perception of “above" and "below" as meaningful categories?

We've also been pleased to discover special opportunities in designing for an interactive, web-based medium and with tools such as Macromedia Flash. For example, Flash smart-clips and symbol libraries allow us to work on many different elements at once in a modular fashion. Small adjustments to symbols can be made at any point, and any 
late-stage changes ripple effortlessly through all our designs. We can build and test individual elements of interactivity (the "actions" of our conceptual model)

independently of our work on the notation system for objects and relations. Of course, the interactive medium alone permits several features of our prototype which we consider fundamental to the representation of temporal subjectivity and interpretation of humanities data - such as draggable layers, adjustable dials, and the nowslider - and will in future enable experiments in three-dimensional and time-based, animated interfaces. Our goal of producing a composition space which humanities scholars might employ as a first step in formal content modelling has spurred us to develop a rich yet carefully regularized and constrained graphic vocabulary for time and temporal relations.

\section{F. In-progress summary and conclusions}

The project aims to produce two related interfaces, which will draw on our specialized visual vocabulary for the representation of temporal relations and subjectivity: an interactive "play" or composition space and a more static, data-driven display space. The display space will enable users to produce large-scale visualizations from existing machine-readable data - generally material from humanities archives that has been marked up, with attention to its temporal qualities, in terms and with structures that can be understood by our own model. Beta tests of the display space will involve material from two University of Virginia digital archives: the Salem Witch Trials Archive (sponsored by the Institute for Advanced Technology in the Humanities) and 
the Yancey Family Papers (part of the Virginia Center for Digital History’s "Race and Place Project”).

Because of its interactive nature, the structure of the composition space lends itself to testing and refining our visual vocabulary even as we develop it. We have therefore begun work on the composition space first, and hope to see it through to a formal beta testing phase in the coming year. We are presently working with graphic designer to establish a workable set of Flash symbols for each type of marker and subjective inflection called for by our model. ${ }^{61}$ A Flash programmer has been involved in the development of the underlying schema that defines and constrains our composition space, and will soon begin to script elements of interactivity using our Flash symbol set. We anticipate beta testing the composition space against concepts drawn from the Salem Witch Trials and Yancey Family projects, as well as with data reflecting the interests of a variety of local scholars and students.

As we have suggested, more research and development is necessary before we can consider enabling the composition and display modes for three-dimensional multivariate diagramming. Beta testing and further research into tense modalities and deixis may reveal a need for a specialized symbol set for modelling linguistic temporality. Indeed, many such "plug-in" symbol sets may be called for, and as we develop our prototype we hope to critique the success of our model's level of generality and the degree of freedom it lends to scholars through built-in user configurable elements. 
The work involved in making possible on-the-fly translation of composition space diagrams into regular expressions and parseable data models is not insignificant. Our research in the field of diagrammatic reasoning and semantics will be critical to this part of the project, as will a long process of trial-and-error testing. However, we feel confident that the production of a visual composition space as a primary tool for content modelling is not only possible, but salutary for the field of humanities computing. The richness and inherent complexity of any formal knowledge representation schemeparticularly any graphic scheme - for time and temporality make this project a perfect testing ground for the rehabilitation of visual thinking and interface into the early developmental stages of humanities computing projects.

\section{G. Select Bibliography}

Allen, J.F. “Time and Time Again: The Many Ways to Represent Time.” International Journal of Intelligent Systems, vol. 6, no. 4 (July 1991), pp. 341-355.

Bronstein, H. "Time Schemes, Order, and Chaos: Periodization and Ideology"” in Time. Order. Chaos: The Study of Time IX. Ed. J.T. Fraser. International Universities Press: Madison, CT., 1998.

Burg, J. et al . "Using Constraint Logic Programming to Analyze the Chronology in A Rose for Emily” Computers and the Humanities 34 (4):377-392, December 2000.

Fraser, J.T. Time, The Familiar Stranger. Massachusetts UP, 1987. 
----. "From Chaos to Conflict," in Time. Order. Chaos: The Study of Time IX. International Universities Press: Madison, CT., 1998.

Gray, M., et al. “Coupling Application Design and User Interface Design.” Proceedings of the ACM Conference on Human Factors in Operating Systems, May 1992; pp. 658 658.

Hoffman, B. and Minas, M. “A Generic Model for Diagram Syntax and Semantics.” In Proceedings of the ICALP2000 Workshop on Graph Transformation and Visual Modelling Techniques, 2000.

Jensen, C. S., et al. “A Glossary of Temporal Database Concepts.” Proceedings of ACM SIGMOD International Conference on Management of Data 23, 1 (March, 1994).

Jordan, P.W. Determining the Temporal Ordering of Events in Discourse. Unpublished masters thesis for Carnegie Mellon Computational Linguistics Program, 1994.

Koeth, O. and Minas, M. "Generating Diagram Editors Providing Free-hand Editing As Well As Syntax-directed Editing.” In Proceedings of the International Workshop on Graph Transformation, 2000.

Kraus, S. et al. Representing and Integrating Multiple Calendars. University of Maryland Technical Report CS-TR-3751, 1996.

Kuhn, W. "Are Displays Maps or Views?" In Proceedings of ACSM-ASPRS Auto-Carto 10, in Baltimore. American Congress on Surveying and Mapping, Vol. 6, 1991; pp. 261 274. 
Kullberg, R. Dynamic Timelines: Visualizing Historical Information in Three Dimensions. Master's thesis, Massachusetts Institute of Technology Media Laboratory, 1995.

Meyer, B. “Constraint Diagram Reasoning.” In Proceedings of the Fifth International Conference on Principles and Practice of Constraint Programming, October 1999.

Meyer, B. et al. “Automatic Construction of Intelligent Diagramming Environments.” In HCI'99: International Conference on Human/Computer Interaction, Munich, Germany, August 1999.

Minas, M. Translating Diagrams. Department of Computer Science II of the University of Erlangen-Nürnberg, Germany. Technical Report 1999.

Narayanan, N.H. and Huebscher, R. "Visual language theory: Towards a Human Computer Interaction Perspective.” In Marriott, K. and Meyer, B. (eds.), Visual Language Theory. Springer, New York; 1998.

O'Toole, M.A. “The Theory of Serialism in The Third Policeman.” Irish University Review. 18(2):215-225. 1988.

Plaisant, C. et al. "Life Lines: Visualizing Personal Histories.” In Proceedings of CHI '96 (Vancouver, Canada, April 1996), ACM Press, 22 1-227.

Price, H. “The View from Nowhen” in Time's Arrow and Archimedes' Point. Oxford UP: New York, 1996.

Renolen, A. "Temporal Maps and Temporal Geographical Information Systems (Review of Research)". Department of Surveying and Mapping (IKO) The Norwegian Institute of Technology. November, 1995; revised February 1997. 
Reynolds, Teri. “Spacetime and Imagetext.” Germanic Review. 73(2):161-74. 1998 Spring.

Schreiber, F.A. "Is Time a Real Time? An Overview of Time Ontology in Informatics" in Real Time Computing, 1992.

Steedman, M. “The Productions of Time.” (draft tutorial notes 2.0: University of Edinburgh. ftp://ftp.cis.upenn.edu/pub/steedman/temporality/).

Theodoulidis, C. et al. "A Conceptual Modelling formalism for Temporal Database Applications.” Information Systems, 16(3):401--416, 1991.

Theodoulidis, C. and Loucopoulos, P. "The Time Dimension in Conceptual Modelling." Information Systems, 16(3):273--300, 1991.

Tufte, Edward, Envisioning Information, Graphics Press: Chesire, CT,1990.

Vila, L. and Schwalb, E. "Revisiting Time and Temporal Incidence.” In Proceedings of the AAAI'96 Workshop on Spatial and Temporal Reasoning, 1996.

Zhou, Q. and Fikes, R. "Reusable Time Ontology". Knowledge Systems Laboratory Technical Report KSL-OO-O 1; Stanford University, February 2000. 


\section{H. Figures}

1a. Linear diagram: John David Miller and John Maeda's Grand Canyon:

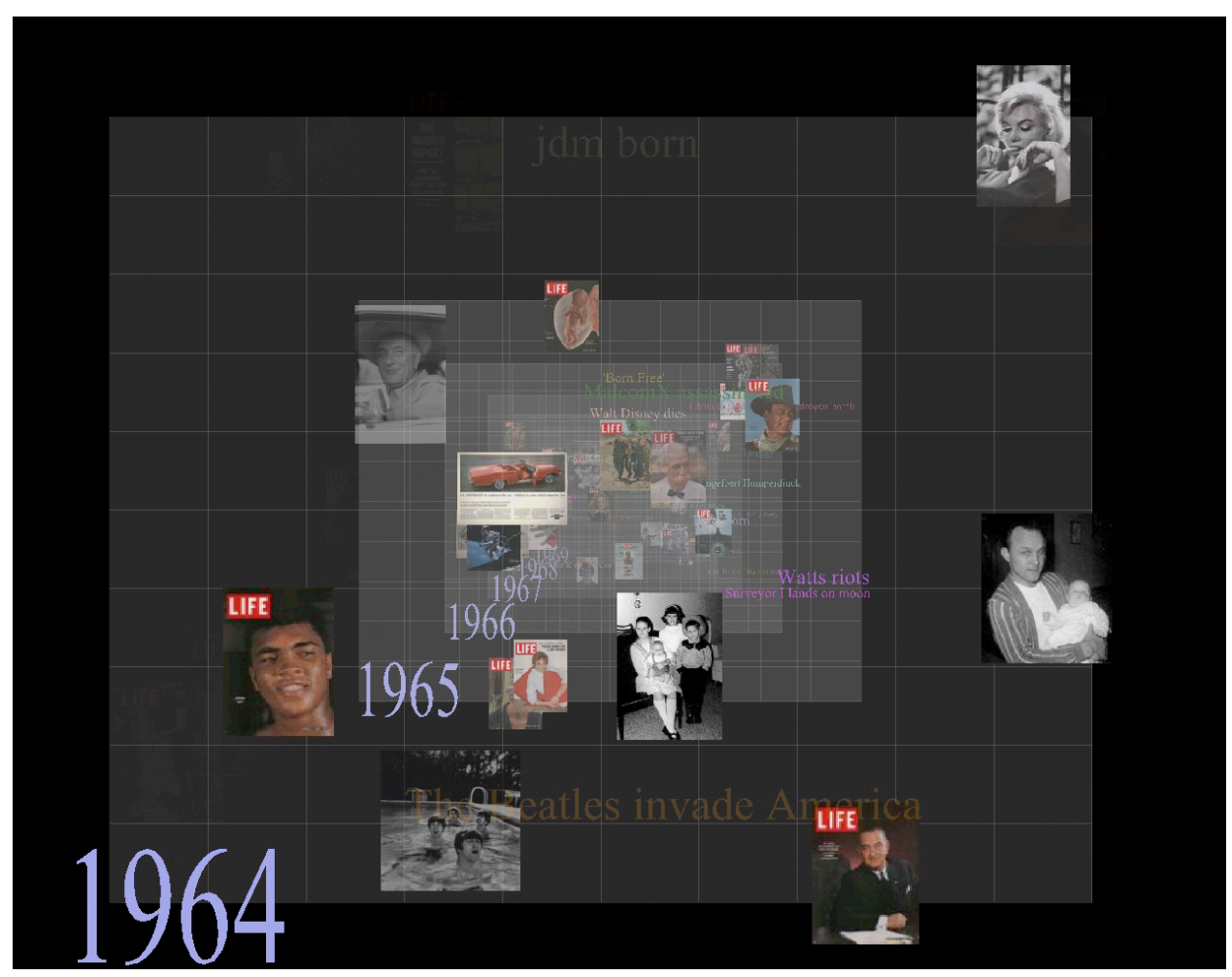


1b. Linear diagram, generated by Bruce Robertson's Historical Event Markup and Linking (HEML):

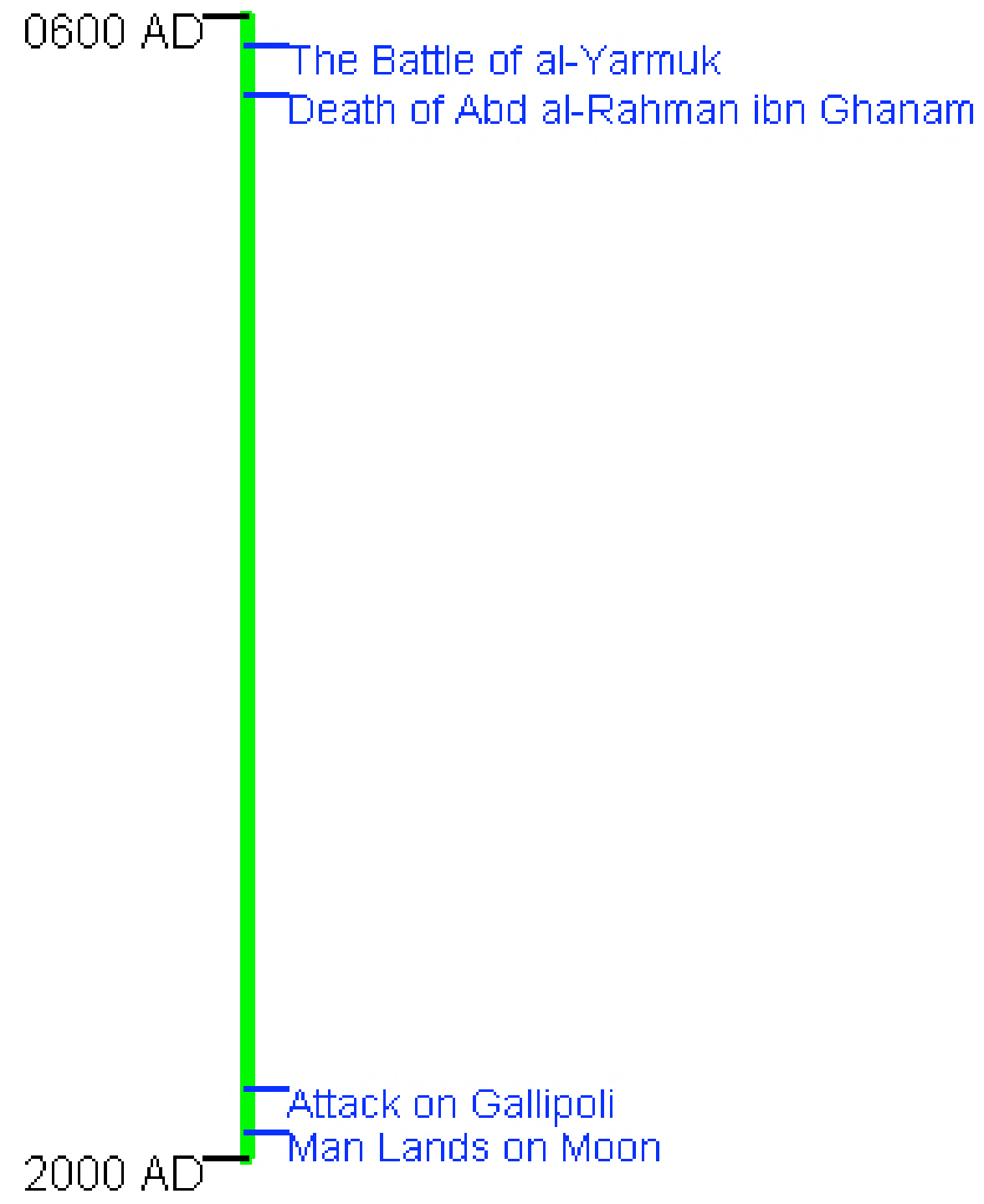


1c. Linear diagrams with Judeo-Christian emphasis. Linear time, 2-dimensional, but inflected by ideological considerations. Time frame is largely event driven. From Bronstein.

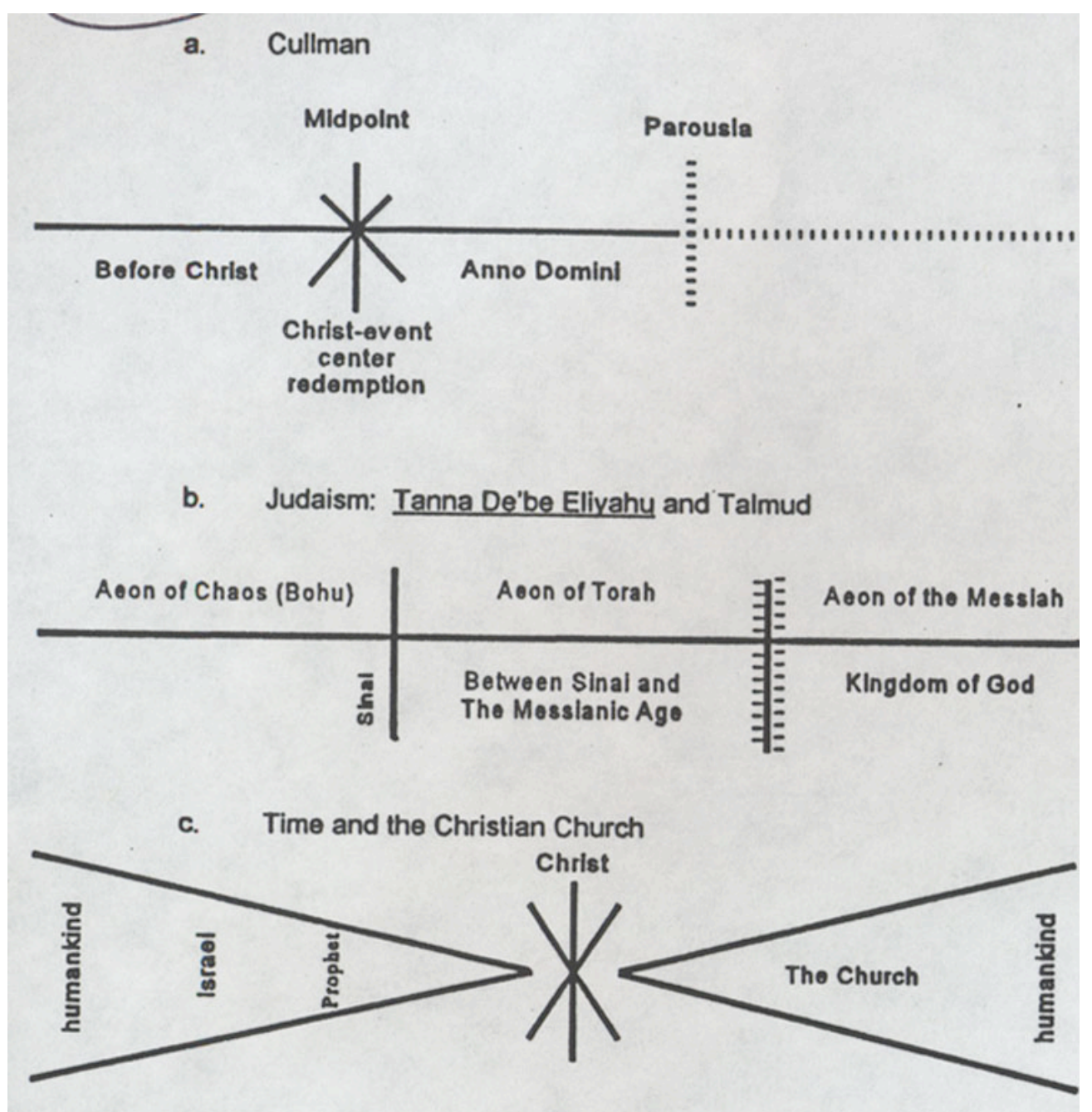

Figure 3.2 Christian and Judaic Periodizations. 
2. Multivariate planar diagram, from the New York Times. Multivariate table using one axis for correspondences (horizontal) and multiple values on horizontal axis for precipitation, temperature, humidity data. Precipitation indicated in discrete units, temperature and humidity in continuous mode. Extrinsic time frame.

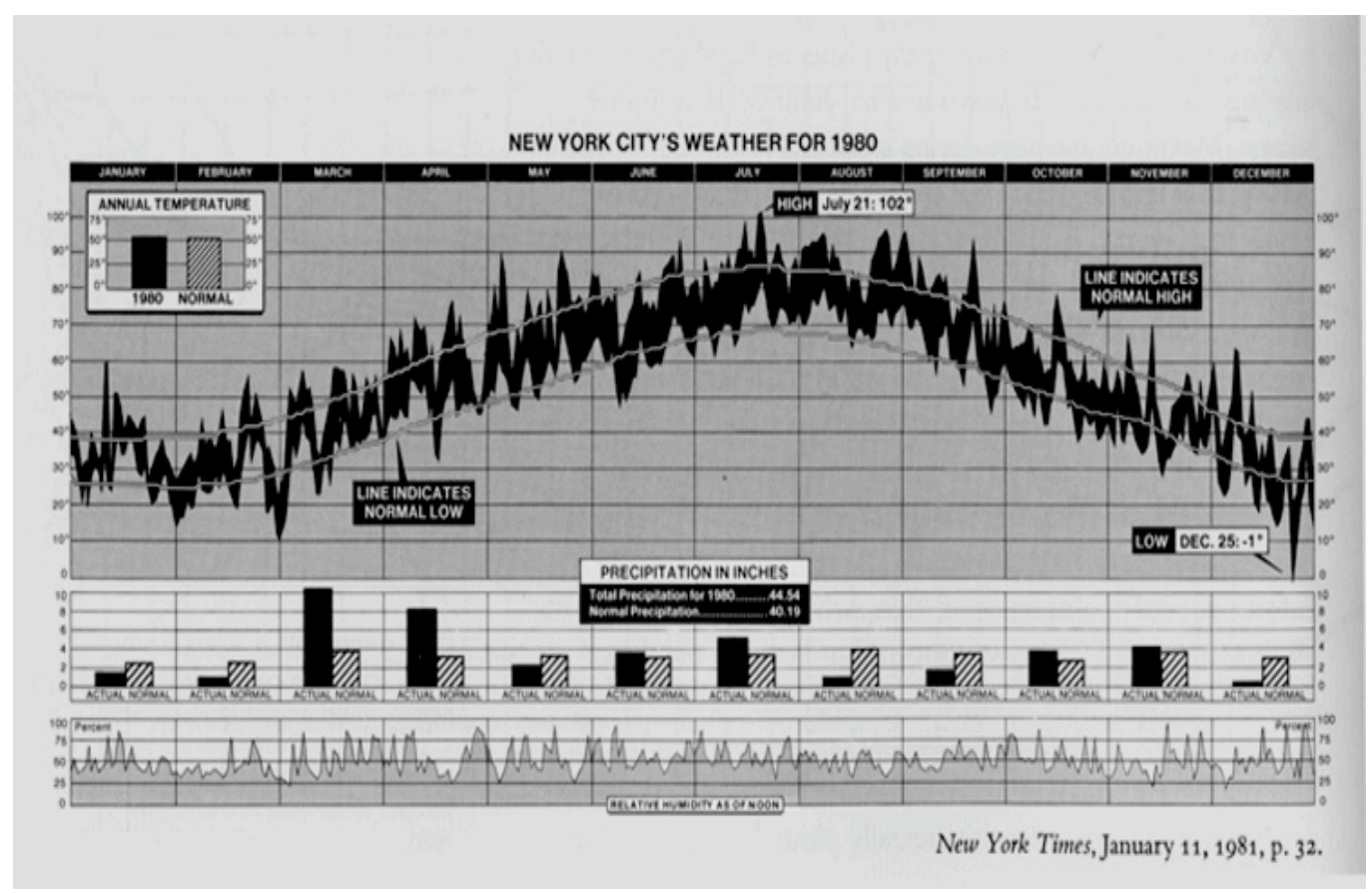


3. Spatial diagram: Hydrocarbon emissions in Southern California. Linear time line in 2.5 dimensions (orthographic). Data shown in continuous display cut snapshot mode. Information is mapped onto a topographic plane. Extrinsic time frame. From Tufte.

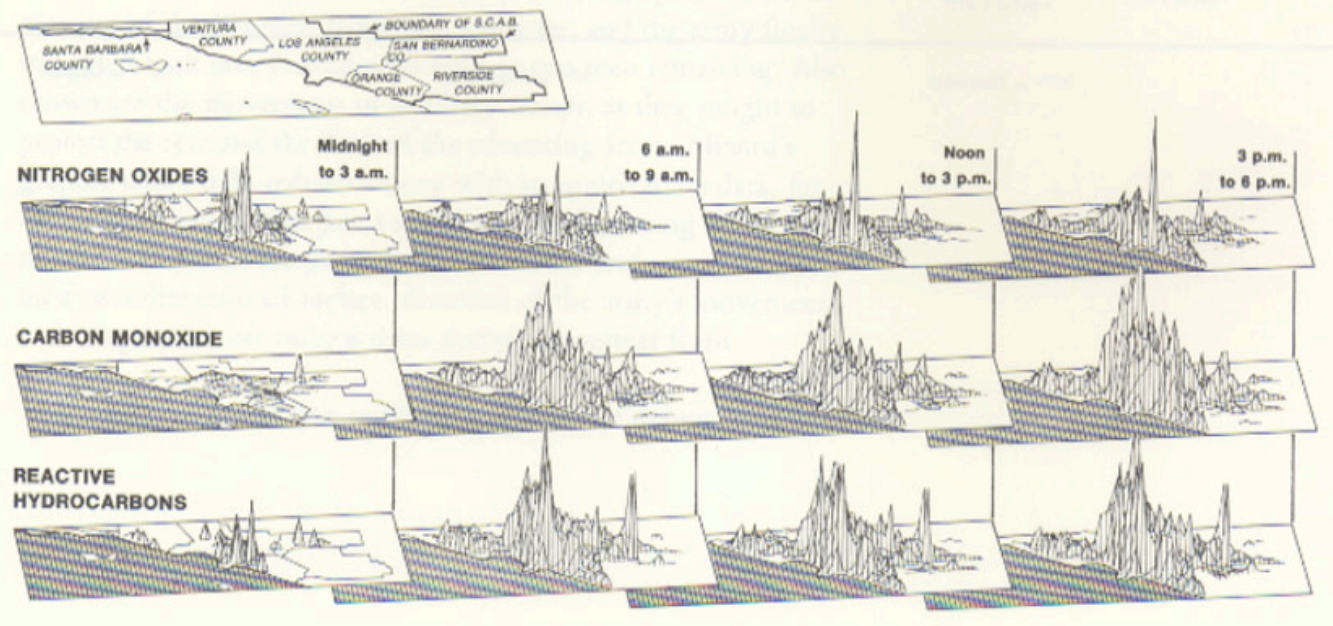




\section{Appendix B. Original Temporal Modelling Software Proposal}

\section{TEMPORAL MODELLING PROJECT Design Specifications}

11 April 2001: BPN

These are the preliminary specs for a complex set of tools being envisioned by the Temporal Modelling project. In essence, the tools would allow a user to design an intuitive, interactive visualization of the temporal relations in any set of data, generate a content model (or DTD) for the data FROM the visualization, mark up and parse the data according to this DTD, and then put the marked data back into the same matrix to generate new visualizations. These tools will be designed in Flash and will utilize the XMLsocket. A rhetorical aim of the project is to promote visualization and interface design as an analytical component of data content modelling.

Steps involved:

A. Work to categorize and define a limited number of visual metaphors for representing time, and analyze the ways they could be placed in juxtaposition in a graphic space. (Examples: linear, cyclical, subjective vs. mechanical time, duration, sequence, 
simultaneity.) This will, in part, be the work of the summer seminar, 18-22 June 2001.

B. Build a paintbox-like set of tools or widgets in Flash using these metaphors. Make it possible for a user to click and drag these widgets onto a stage and design a visual representation of temporality in his subject of study. Allow the user to name the elements he chooses and define their relationships to his data. (Note: At this point, the widgets will have no connection to a dataset in the computer. That is, the user's visualization will be intuitive - meant to aid in markup, not to process and represent marked data: i.e. "this is how it seems to me the temporal aspects of this novel should appear." This is a conscious alternative to the usual process of markup or database building, in which visualization comes as a last step.)

C. Add to this preliminary toolbox a chat application (can be done in Flash over the XMLsocket; see an example at www.moock.org) for collaborative work and the ability to store a set number of visualizations on the server for each user. Set up password accounts with a certain quota of disk space.

D. As a next step, allow the user to export his visualization and its labelled elements in the form of an XML DTD. We should allow some tweaking of the DTD (maybe even design a Flash editor so that this can be done in the same environment as the visualizations), as long as it continues to parse and conform to certain standards that 
our visualization widgets can handle.

E. Send the user off to tag his data according to the visually-generated DTD. Again, it may be possible for us to design an editor so that the tagging can be done on-site and stored via the socket on our server.

F. The second visualization step involves uploading the user's tagged, parsed data and automatically applying the same paintbox set to generate a visualization. This time, the process will work in the conventional way, but the user will be able to compare this visualization with his first, intuited picture to troubleshoot and study the differences. All of these components work together to make a tool applicable for teaching and interpretation, database-building, visualization research, and data analysis. 


\section{Appendix C. Rossetti Archive Redesign Proposals ${ }^{62}$}

\section{A. MODES/TOOLS PROPOSAL}

This is a proposal for a Rossetti interface based on tools or modes of inquiry rather than on types of content, presented to the redesign team on 3 August 2003 by Bethany Nowviskie.

\section{Background:}

The Rossetti Archive interface is currently organized according to content type (ie. poem, prose, painting, doublework). Simple chronological and alphabetical lists and a rudimentary search engine make individual RAWs and RAPs/RADs accessible within those categories. RAPs and RADs are subordinated to RAWs. This over-arching organizational scheme of content types (like many other features of the Archive, such as filenames) reflects in part a structure set up to aid the Archive's creators in locating and creating files in the absence of a user interface. It does not necessarily reflect our understanding of our users' needs and desires. Just as, over time, we've come to appreciate that the RA is more an interpretive/interpreted collection than an objective archive, we are coming to the realization that its real work lies in facilitating user interpretations and that its structures must support that work explicitly. 


\section{Problem:}

A content-based organizational scheme assumes that users come to the Archive with some pre-knowledge about Rossetti's works, so that they can look in the proper categories for files, or that they already know work/doc titles or other information when using the search engine. It otherwise assumes that users unfamiliar with Rossetti have a high tolerance for browsing and reading on the screen.

It also assumes that they come to the Archive in search of DGR content - that they wish to use the RA like a library's reading-room (where one's access to documents is good, but where options for working with them are limited). Such assumptions imply that the interface needs to be nothing more than a card catalog.

This model is not working. Too few users have the requisite level of familiarity with DGR and his works (or the desire simply to access RA files) to make this organizational scheme useful. More importantly, the scheme runs counter to our desire to demonstrate the applicability (and even enhancing quality) of digital resources to traditional interpretive practices in the humanities. We don't want to give the impression that we are only a storehouse for content, or that all RA markup only contributes toward simple searches and HTML formatting on the screen.

\section{Proposal:}

We should base the primary Rossetti Archive interface on interpretive tools and modes 
of inquiry rather than on types of DGR content, as a means of demonstrating the richness of the Archive and its ability to be applied to innovative and traditional academic methods. The visualization tools and clever indexing methods we've previously imagined as solutions to the problem of the current interface's opacity only go so far, as they are meant to make a statement about the Archive (namely, that this collection is large and complex). Rather than making statements, we should be provoking questions.

Some primary tool-based and modal interface divisions (based on projects and efforts or interests currently in play at SpecLab) might look like this:

\section{Temporal Modelling}

The Iconic Page

Discourse Fields

Ludic Method

Traditional Modes

Temporal Modelling could house ways of searching, visualizing, and theorizing about DGR works and docs in terms of their temporal qualities: relation of artifacts in the Archive to Victorian history, DGR's biography, the Pre-Raph scene, relation of individual docs and pics to the evolving concept of a work, calendrical and genealogical/stemmatic views of composition/printing histories, user-constructed 
models of all of the above and critical (internal) interpretations of imaginative works using PlaySpace tools and inflections.

The Iconic Page could make use of the 'Patacritical Demon concept and other imagebased tools to facilitate the study of DGR's pictorial works and of the graphical features of textual documents. Perhaps we can also enable and publish user-interpretations that take graphical form.

Discourse Fields could include tools based on Ivanhoe's (eventual) solution to the problem of discourse field display, navigation, and editing/annotation. In addition, it might include features of use to an interpretive community, like discussion lists (perhaps in partnership with ITC/Toolkit). Andrea Laue's work may be relevant in this section.

Ludic Method is a catch-all category for more experimental, game-like interpretive tools, including a link to the Ivanhoe Game (preferably a way to import and play with RA texts in Ivanhoe) and other experiments (possibly including Llullian wheels, kenning games, a Nomic engine, and other Glass Bead variants, etc.). Steve Ramsay’s deformance engines would be appropriately placed here.

Traditional Modes (in need of a better name) is the place where users will find (and be able to contribute to, through the vetting mechanism of an editorial board) more familiar, largely prose-based interpretations. Curator-made Archive exhibits, occasional 
essays, related journal articles, maybe even Jerry’s top-level commentaries would all belong here. We could also make the pre-existing content-type organizational scheme available to users in such a space.

\section{Needs:}

Patience: Many of these tools are currently in the works or pre-exist in other forms and would need extensive adaptation for our purposes. This will take time. We'll need either to tolerate a long period with little progress to show on the screen or (my preference) to make some usability modifications to the content-based interface while the tools initiative is underway. This won't be wasted work, if we intend to make content-based access possible among the "Traditional Modes."

Search Engine: Critical to the success of this idea is a flexible search engine, which could make RA content (RADs, RAPs, RAWs) accessible as appropriate in each of the tool/mode categories. We can't sacrifice content accessibility in this modal approach; it's not a modes-over-content choice, but rather a demonstration of the flexibility of the content. We should explore Cerisent and Clara Yu's semantic indexing engine. Many of the tools proposed here will require not only access to RA files, but also the ability to import data for manipulation and display.

Specifications: Each mode/division needs to be thoroughly theorized, and we must write clear specifications (and use cases?) for each tool. 
Expertise: We'll want to identify skills and interests we have in house and hiring needs as soon as possible. We should think about reasonable partnerships, for example with ITC, Romantic Circles, and the University Press of Virginia.

\section{B. DEVELOPMENT TRACKS PROPOSAL}

This proposal, outlining two development tracks (pedagogical/community-building and speculative) was presented to the Rossetti redesign team by Bethany Nowviskie in January of 2004.

A Rossetti Archive Redesign will make existing materials more usable and relevant in classroom, research, and networked discussion contexts, while simultaneously demonstrating through a speculative showcase that digital resources like the Archive can move beyond pre-critical problems of access and dissemination by responding to the interpretive needs of scholarship in flexible and provocative ways.

\section{Redesign Job One: Supporting Pedagogy and the Scholarly Community}

This work will be our highest priority. Its goal is to increase the usability of what we've already got and respond with new tools to the current perceived needs and desires of the scholarly community, especially as concerns classroom use of the Archive and new 
models of academic collaboration and communication. Ultimately, the redesigned Archive will mesh better with the established social practices of literary scholarship and be enlivened by user participation.

\section{Some First Steps:}

Solicit feedback from local faculty and maybe even Victoria list readers on the current state of the Archive and the suggested new features below.

Clarify role of the editorial board and its willingness to be involved in selecting

\& assisting curators and in peer-reviewing user-generated materials.

Flesh out feature list below and specify programming, design, and

social/organizational tasks and degree to which these features overlap with 9s efforts.

Examine current ARP \& Rossetti staff commitments and determine whether new hiring is necessary and also what new equipment and software is needed. Determine quickly which of the efforts outlined below can run concurrently and whether any minimal refinements to RA markup will be necessary for Job One features. (These will take precedence over any tweaks on subsets of Archive material for "Speculative Work" described on the next page.)

\section{Some Features:}

The curator concept: we will invite certain scholars to make and publish annotated, selected arrangements or "interfaces" to the Archive in order to 
highlight different interpretive perspectives on the same body of material.

Critical resource gathering: we should publish occasional essays ourselves (either invited or peer-reviewed by the Rossetti editorial board), and assemble and reprint as possible (or link to) related journal articles, CFPs, conference proceedings, discussion groups, print bibliographies etc. We should also include a mechanism for solicting resource suggestions from users toward the making of a grand, annotated (and user-annotable) bibliography.

Sophisticated discussion tools: we will build or incorporate existing tools to make online discussions in both informal and formal venues (as desired: chat, personal scholarly blogs, threaded discussion forums, email lists, sponsored or “edited” discussions, etc.) more rigorous, archived, searchable, and citable.

Basic organizational work: we must analyze and address problems in the presentation of RAD/RAP/RAW relations and organizational indices (alphalists, etc), and evaluate the need for a tools/modes-based approach to organization as opposed to a content-based scheme.

Improved search engine: minimally, we will implement novice and expert modes and address the extent to which our existing metadata is being exploited, but the design of more sophisticated browsing or "card-catalog" features are also desirable here.

The marginalia concept: we will create a means for users to annotate or comment on Archive files and for those notations to be made (optionally) visible either as sponsored, peer-reviewed additions or in an open, free-for-all types 
way.

Configurable editions: the reader should be able to choose how tex ts are displayed - over-arching organizational schemes, display of transcripts or images, edited, unedited, showing/hiding variants \& commentary, etc. through what is essentially an arrange-your-own archive feature. These preferences and schemes must be stored \& editable by logged-in users. We also will desire simple ways to make vetted user-edited versions (preferences, comments, readings) public and able to be incorporated into other users' versions (this may involve simple "cloning" of models or necessitate complex inheritance rules).

Screen-to-Print initiative: we will partner with print-on-demand services (or at least enable nice PDF formatting for private printing) of user-specified arrangements, commentaries, etc, primarily so that instructors can make course packets. (This is something 9s should do and Rossetti may showcase on a small scale — because the demand for Rossetti-only packets will be small.)

Calculating Variants: Even displaying a simple codex-like apparatus that could appear in a print edition would be an advance for the Archive, which currently takes what Peter Robinson describes as a backward editorial step by just presenting multiple texts and leaving readers to collate them on their own. The 9s Juxta tool may be meant to solve this problem, but a preliminary Rossetti redesign could do with in-line notation $\&$ links to variant readings. 


\section{Speculative Work: Showcasing Toolsets for Interpretive Application}

An important sideline to Job One tasks, this work may not necessarily produce finished tools, but may instead take the form of testbed or showcase applications - open-source examples and "imaginary solutions" meant to provoke further development. Specialized editorial, visual, and transformative tools fall in this category.

\section{Some First Steps:}

Clarify for all Redesign participants the distinction between Job One and Speculative efforts, then make discussion of the latter a regular feature of SpecLab meetings.

Likewise solicit and refine ideas through periodic contact with our extended SpecLab family and Humanist listmembers, etc (running on steam from ACH/ALLC “New Directions”).

Determine degree to which Rossetti Archive will partner with or showcase outside efforts (for example, 9s/ARP work on the Ivanhoe Game, Juxta, and the Page Turner) and the related SpecLab Temporal Modelling and Demon projects.

Build and publish an open-house space on the RA site for ongoing speculative work and incorporate discussion tools here as they emerge from Job One. Collect some readings and reviews on the subject and publish or annotate 
references to them.

Cultivate partnerships with other groups and individuals doing similar work (Sinclair, Ramsay, TAPoR, LOOKSEE, HEML, Fry, Maeda, etc.) and explore possibility of hosting a bibliography or clearinghouse.

\section{Some Possible Directions:}

specialized editoral applications for highlighting variants and manipulating texts (like our own Juxta Render and Juxta Author)

implement a real image-based edition? utilize the page-turner interface? (my sense is that the latter may have theoretical problems that could be best addressed in a showcase environment)

problems in finding and illuminating patterns in large text archives, and in making pattern-finding tools available to users

addressing the temporal axis: can we show a work's text changing over time? can we display or analyse the changing graphic character of its witnesses? experiments with overlapping hierarchies, even just as illustrations of the problem and explorations of existing proposed solutions (Concur, MECs, etc.) implement a Temporal Modelling-like PlaySpace for making timelines and other (possibly non-temporal) diagrams, then exporting a re-purposable XML rendition from visualizations: these might basically be tools for glass bead games, but we could also take Temp Mod's “modelling” architecture further. 
incorporating tools and ideas from our work on the 'Patacritical Demon and

Ivanhoe Game

visualization of internal relations in the archive and of patterns of use

incorporation of other pre-existing text analysis and deformative tools (Stephan

Sinclair, Ben Fry, etc.) 


\section{NOTES}

${ }^{1}$ Some seventy medieval manuscripts survive of the Ars Brevis, a shortened expression of Llull's tools and methods, alone, and Anthony Bonner records 24 Renaissance editions of this popular work (Bonner 577). For a succinct reception history, see Bonner's introductions to "Llull's Thought" and "Llull's Influence" in his Selected Works of Ramon Llull (1232-1316). vol 1. Princeton UP: 1985.

${ }^{2}$ All images of Llull's figures and mechanisms presented here are taken from Bonner's reproduction of the Ars Brevis, (Escorial, MS f-IV-12, folios 3, 4, 6, and 7) in the Selected Works.

${ }^{3}$ Other notions of "visualization" are possible. My work on the Temporal Modelling project is an extended examination of the viability of one of these alternatives: that we may think of visualizations not as revelatory of abstract information somehow already "embedded" in data, but instead as part of a process of composition and experimentation with data graphics.

4. This state of affairs is changing, although a brief flurry of activity centering around the LOOKSEE project and other online venues seems to have subsided. See Matthew 
Kirschenbaum's special issue of Computers and the Humanities (36.1: 2002) on "ImageBased Humanities Computing” (to which I contributed a selected bibliography) and Johanna Drucker's “Digital Ontologies: The Ideality of Form in/and Code Storage -orCan Graphesis Challenge Mathesis?” in Leonardo. 34.2 (2001): 141-145.

${ }^{5}$ See Figure III.1 and a brief discussion of "Rossetti Spaghetti" in Section F of Chapter III, on the Ivanhoe Game. Any number of algorithmically-generated visualizations in humanities computing contexts bear these observations out.

${ }^{6}$ Algorithmic text analysis tools such as those designed by Stephan Sinclair in an OuLiPian mode are now being aggregated (among less consciously ludic tools) under the auspices of TAPoR, the Canadian Text Analysis Portal for Research, directed by Geoffrey Rockwell. See http://huco.ualberta.ca/Tapor/. Paley’s TextArc project is available at http://www.textarc.org. Benjamin Fry's work at the MIT Media Lab and elsewhere is available at http://acg.media.mit.edu/people/fry/. See especially his genomic cartography and organic information design projects, both of which have been applied to text analysis (but, unhappily, only in art installation contexts largely ignored by "serious" textual scholars).

${ }^{7}$ Interestingly, Florian Cramer points out that Friedrich Schlegel, in the 1790s, defined Romanticism in terms of recursion and formal self-reflexivity - the same terms under 
which contemporary algorithmic and combinatorial digital art (of which Cramer himself is a Lullian practitioner) takes shape. See Cramer's talk, “On Literature and Systems Theory,” of April 2001 online:

http://userpage.fu-berlin.de/ cantsin/homepage/\#theory

${ }^{8}$ And it is no new notion; see the discussion of constraint in the work of Dante Aligheri in Jerome McGann and Lisa Samuels' seminal essay, "Deformance and Interpretation.”

${ }^{9}$ This idea is closely tied to the biology of autopoiesis as articulated by Varela and Maturana. See their Autopoiesis and Cognition. The Realization of Living(D. Reidel Publishing Co.: Boston, 1980) and a brief discussion in Chapter III.

${ }^{10}$ The difficulties involved in the rigorous analysis of this quality of poetic production have been framed as a "problem of overlapping hierarchies" by the humanities and linguistic computing communities. See for instance Sperberg-McQueen’s comments at the Extreme Markup Conference 2002 ("What Matters?" http://www.w3.org/People/cmsmcq/2002/whatmatters.html) and discussion by Renear and McGann at ACH/ALLC 1999 ("What is Text?").

${ }^{11}$ See Marjorie Perloff's discussion of aleatoric and generative procedures in John Cage's Roaratorio for a more nuanced view of this artist's work (Radical Artifice 150- 
$161)$.

12 The important notion that any algorithm's output, its text, naturally opens itself up to multiple subjective interpretations and acts of complicity or resistance by the readers who (re)constitute it played no part in OuLiPo's critique, which instead came down on exclusively methodological lines. It is also important to note that other critics of Dada and Surrealism see more subjective license in the production of procedural art (cf. Dawn Ades on collage and photomontage, Robert Motherwell on Dada, Marjorie Perloff s wide-ranging work on Cage and others, and Gregory Battcock’s anthology of minimalism).

${ }^{13}$ Often, but not always. Conceptual artists like Sol LeWitt provide us with explict instructions - algorithms — for producing works they may never execute on their own. See LeWitt's recent installation at the Tate Modern, "Fifteen part Drawing using four Colours and all Variations (straight parallel Lines, each Colour in a different Direction) 1970," which was executed in absentia from a simple set of combinatorial and constructive rules.

${ }^{14}$ On the relation between hermeneutics and design, a developing interest in the architectural community, see especially Schön, Snodgrass ("Is Designing Hermeneutical?”), Coyne, and Chalmers. See also Cross's “Designerly Ways of 
Knowing” and Winograd and Flores:

We also consider design in relation to systematic domains of human activity, where the objects of concern are formal structures and the rules for manipulating them. The challenge posed here for design is not simply to create tools that accurately reflect existing domains, but to provide for the creation of new domains. Design serves simultaneously to bring forth and to transform the objects, relations, and regularities of the world of our concerns. Understanding Computers and Cognition: A New Foundation for Design, 12)

15 The NINES project now emerging at the University of Virginia (a "networked interface for nineteenth-century electronic scholarship") promises to address this problem, and may ultimately include versions of the Temporal Modelling and Ivanhoe tools described here. (See http://faustroll.clas.virginia.edu/nines.) See also Appendix C and rossetti (re)design (at http://faustroll.clas.virginia.edu/mt-static/rossetti/design) for materials relating to my work in redesigning the Rossetti Archive to promote interpretive engagement and to make evidence of that engagement manifest in the Archive's data structure.

${ }^{16}$ Chris Crawford, author of the first major handbook for computer game design, contends that all great designers must think algorithmically, concentrating on process over fact and on trend over instance. The antithesis of "algorithmic thinking," he 
writes, is "instantial thinking," which always leads to poor interactive designs. The instantial thinker "comes up with great cut scenes," the passive, movie-like animations that close chapters or levels in many digital action games, "but lousy interactions," which are the heart of gameplay, and "when he designs an adventure game, [the instantial thinker] loves to cook up strange dilemmas in which to place the player, but the idea of a dilemma-generating algorithm is lost on him" (Crawford).

${ }^{17}$ See, for instance, Peter Suber's "Nomic” a game described in section E of Chapter III and on which Imaginary Solution \#1: Dr. Kremlin's Disc draws.

${ }^{18}$ Bonner points out that the word art was the "usual scholastic translation" for the Greek $\tau \varepsilon \chi v \eta$. Llull's work is best understood as a "technique; it was not a body of doctrine, but a system. Or to put it in contemporary terms, it was a structure" (Bonner $62)$.

19 Anthony Bonner suggests that Llullian alphabetic ciphers are constants rather than variables (“What Was Llull Up To?”). Clearly, the wheels and their primitives open themselves to adjustment by a human user or artista. I therefore take this assertion to mean that the letters, once placed in the practical matrix of Llull's wheels and charts, are best understood as having a one-to-one relationship with the objects or ideas they represent, the better to enable the sort of dynamic, performative interaction of an artista 
with a diagram Llull favored.

${ }^{20}$ Figure 8 was adapted by Anthony Bonner from Palma, Bibl. Publ. MS 1029, fol. $23^{\mathrm{v}}$ and reproduced in his Selected Works of Ramon Llull(vol.2, plate xix).

${ }^{21}$ For an expanded discussion of visualization and diagrammatic reasoning in the context of the Temporal Modelling Project see section D 1 of chapter II.

${ }^{22}$ It is for this reason that I prefer the terms "environment," "instrument," and "mechanism" to "tool" when positing mechanical or algorithmic aids to humanities interpretation. An environment is by definition an inhabitable space. Instruments are played as well as used. And a mechanism is a system that can be opened up for analysis and adjustment. A “tool," on the other hand, seems too self-contained and inviolable an object for my taste.

${ }^{23}$ Johanna Drucker's ongoing "Metalogics of the Book" project is one attempt to address this problem.

${ }^{24}$ A notable exception to this older trend is the work of maverick analytical bibliographer Randall McLeod, who comfortably straddles empirical and interpretive genres in the same way that writers like Susan Howe blend poetic practice and criticism. 
${ }^{25}$ For more on the inner standing point, see here section D.3 of Chapter II and section H of Chapter III as well as the discussion of “Jenny” in Jerome McGann's Dante Gabriel Rossetti and the Game that Must Be Lost. An excerpt from the latter appears in the first of a set of Ivanhoe Game moves reproduced in Chapter III.

${ }^{26}$ The storyboard, prepared to provoke discussion at the summer seminar, is available among other historical documents at the Temporal Modelling Project's website: http://www.iath.virginia.edu/time. The software specification, which I drafted in response to conversations with Johanna Drucker and others, and later refined in consultation with IATH staff members Worthy Martin, Steve Ramsay, Kirk Hastings, Chris Jessee, and John Unsworth, is reproduced in Appendix B. Some aspects of this original plan later proved unfeasible or undesirable (such as the automatic generation of a DTD), but it is important to note that both the rhetorical and practical goals of the project were clear even at this early stage.

27 The full seminar packet is available on the Temporal Modelling Project's website, at http://www.iath.virginia.edu/time/research/seminar.html. University of Virginia readers may also access many of our selected readings in their entirety through the ITC Toolkit page for the seminar. 
${ }^{28}$ Although we were conscious of the richness of other structuring metaphors (for instance, the inhabited “canyon” of Maeda and Miller's Grand Canyon software offers a more compelling vision of lived temporal relations), we decided to begin practical implementation of the PlaySpace with the simplest structure possible: a linear axis, or timeline, hoping later to expand into cyclical, calendrical, geospatial, and other time systems. Imaginary Solution \#2: Temporal Modelling at Sea exposes some limitations imposed on PlaySpace models by such early decisions.

${ }^{29}$ Cf. Appendix A, Figure 4 for the version of this table submitted to Information Design Journal in an article co-authored with Johanna Drucker, November 2001.

${ }^{30}$ For more on modelling in the humanities (especially diagrammatic modelling), see Willard McCarty’s online resources, hosted by King’s College, London.

${ }^{31}$ I term this ability "illusory" only because, in fact, the system of affordances and constraints built into the PlaySpace ensures that all visual models are constructed simultaneous with their XML representation. No AI construct is necessary to "extract" predicates from a visualization - the PlaySpace user provides that fundamental brainwork in his interaction with the tool.

32 On the 'Patacritical Demon, see Jerome McGann’s “Marking Texts of Many 
Dimensions" in the forthcoming Blackwell's anthology, A Companion to Digital Humanities.

33 The subjective nature of temporal perception is a recognized problem in subject areas which may seem far removed from humanities interpretation, but which in fact make use of the concepts and counter-assumptions regarding time outlined in Appendix A. For instance, University of Virginia professor Stephanie Guerlain’s students undertake design exercises in subjective temporal visualization of medical procedures as part of their HCI engineering curriculum.

${ }^{34}$ Working prototypes of these nowsliders can be examined and manipulated at http://www.iath.virginia.edu/time/storyboard. Nowsliders only become meaningful as networked visualizations of temporal relations when they are in motion, so static screenshots like those in Figures 15 and 16 are not particularly expressive. The reader is, however, enfranchised to take scissors to this dissertation and assemble an animated flip book from the dozen slices he can make of Figure 16.

${ }^{35}$ SpecLab, the University of Virginia’s Speculative Computing Lab, came into being as a research collective affiliated with the UVA Media Studies Program at the intersection of the two major humanities computing projects outlined here: Temporal Modelling and the Ivanhoe Game. For more information, including a list of affiliated researchers, links 
to related projects, and a statement of purpose, see:

http://www.speculativecomputing.org.

${ }^{36}$ See especially the conclusion and appendix to Jerome McGann’s Radiant Textuality, the SpecLab web page for Ivanhoe (http://www.speculativecomputing.org/ivanhoe) and a forthcoming special issue of Text Technology on the game, edited by Geoffrey Rockwell, and with contributions by Rockwell, McGann, Drucker, Sansing, and Nowviskie. McGann's essay in this latter collection outlines most clearly the distinction between the larger IVANHOE concept and its manifestation as the Ivanhoe Game.

37 "Jenny" is a dramatic monologue in verse, first composed in 1848 and revised for publication in Rossetti's 1870 Poems. It is a meditation, from an "inner standing point," on the condition of urban prostitutes in Victorian England. The speaker is an intellectual - at once contemplative, guilty, and diffident - whose evening's companion, Jenny, has fallen asleep in his lap. He muses on her resemblance to his beloved cousin, Nell, ("Two sister vessels. Here is one. / It makes a goblin of the sun.”), examines her apartments minutely, imagines her past and future, and ultimately leaves her with her hair full of coins: "I think I see you when you wake, / And rub your eyes for me and shake / My gold, in rising, from your hair, / A Danaë for a moment there." My own moves in the "Jenny" game played in the summer of 2004 with Jerome McGann and 
Andrea Laue are reproduced throughout this chapter, as they bear on themes integral to the discussion, including the notion of a specifically-imagined, subjectively-inflected virtual reality. The text of the game in its entirety is available on SpecLab's Ivanhoe web page: http://www.speculativecompting.org/ivanhoe.

${ }^{38}$ See especially Johanna Drucker's “aesthetic provocation” to the development group, Spring 2003, available in PowerPoint format on the SpecLab site: http://www.speculativecomputing.org/ivanhoe/design.html

39 See my discussion in Chapter I above, section B ("Performance and Interpretation").

40 The subject-oriented approach first developed by Harrison and Ossher and other IBM researchers has more recently evolved into a better-established practice of "aspectoriented programming," of which the AspectJ language is a primary tool. An identical interest in multiple purposes and perspectives, or "cross-cutting concerns," is evident in both subject- and aspect-oriented work, and an argument can be made that they are competing names for the same technology. See Elrad et al, “Aspect-Oriented Programming: Introduction” in Communications of the ACM 44:10 (2001) pp 28-32.

${ }^{41}$ Thanks are due here to Professor Chip Tucker for the reference and the pun. 
42 According to Alfred Jarry, 'Pataphysics is:

above all, the science of the particular, despite the common opinion that the only science is that of the general. Pataphysics will examine the laws governing exceptions, and will explain the universe supplementary to this one; or, less ambitiously, will describe a universe which can be — and perhaps should be envisaged in the place of the traditional one, since the laws that are supposed to have been discovered in the traditional universe are also correlations of exceptions, albeit more frequent ones, but in any case accidental data which, reduced to the status of unexceptional exceptions, possess no longer even the virtue of originality.

(Alfred Jarry, Exploits and Opinions of Doctor Faustroll, 'Pataphysician, Book II, Chapter 8 (1907) tr. Simon Watson-Taylor)

In the same passage, he offers the following definition: "Pataphysics is the science of imaginary solutions, which symbolically attributes the properties of objects, described by their virtuality, to their lineaments." I have punctuated the chapters of this dissertation with three such specifically-imagined "imaginary solutions."

${ }^{43}$ See her essay, "Speculative Computing: Aesthetic Provocation in Humanities Computing" (to which I contributed a discussion of Temporal Modelling) in Blackwell's A Companion to Digital Humanities (forthcoming 2004), eds. Unsworth et al. 


\section{NOTES TO APPENDIX A (by Johanna Drucker and Bethany Nowviskie)}

44 Temporal Modelling is an Intel Sponsored Research Project of the Speculative Computing Lab and Media Studies at University of Virginia. Demonstrations, work in progress, and research reports are available at http://www.iath.virginia.edu/time.

${ }^{45}$ For an overview of some of these issues: Stuart K. Card, Jack D. Mackinlay, Ben Shneiderman, Readings in Information Visualization, Using Vision to Think, Morgan Kaufmann Publishers, Inc., San Francisco, 1999.

46 Fraser, J.T. Time, The Familiar Stranger. Massachusetts UP, 1987 and Schreiber, F.A. "Is Time a Real Time? An Overview of Time Ontology in Informatics” in Real Time Computing, 1992.

47 Schreiber, op.cit.

48 Schreiber, op.cit.

49 Patterns of human activity, even belief systems grounded in cyclic progression towards enlightenment, prove, on examination, to be time arrows "wrapped" on a circular form.

50 Fraser, op.cit.

51 Allen, J.F. “Time and Time Again: The Many Ways to Represent Time.” International Journal of Intelligent Systems, vol. 6, no. 4 (July 1991), pp. 341-355.

52 Jensen, C. S., et al. “A Glossary of Temporal Database Concepts.” Proceedings of ACM SIGMOD International Conference on Management of Data 23, 1 (March, 1994).

53 Steedman, M. “The Productions of Time.” (draft tutorial notes 2.0: University of Edinburgh. ftp://ftp.cis.upenn.edu/pub/steedman/temporality/).

54 Burg, J. et al. "Using Constraint Logic Programming to Analyze the Chronology in A Rose for Emily" Computers and the Humanities 34 (4):377-392, December 2000.

55 Jordan, P.W. Determining the Temporal Ordering of Events in Discourse. Unpublished masters thesis for Carnegie Mellon Computational Linguistics Program, 1994.

56 Bronstein, H. “Time Schemes, Order, and Chaos: Periodization and Ideology"” in Time. Order. Chaos: The Study of Time IX. Ed. J.T. Fraser. International Universities Press: Madison, CT., 1998.

57 Ira Bashow, Seminar Presentation, University of Virginia, June 2001.

58 Price, H. "The View from Nowhen” in Time's Arrow and Archimedes' Point. Oxford UP: New York, 
1996.

59 Reynolds, Teri. “Spacetime and Imagetext." Germanic Review. 73(2):161-74. 1998 Spring.

60 O'Toole, M.A. “The Theory of Serialism in The Third Policeman.” Irish University Review. 18(2):215225. 1988 .

${ }^{61}$ Petra Michel is the graphic designer working on the project. Jim Allman is the Flash programmer.

${ }^{62}$ See also my "Rossetti (re)Design" weblog, an ongoing reflective and practical journal at http://faustroll.clas.virginia.edu/mt-static/rossetti/design. News and updates are available at the "Rossetti Report:" http://faustroll.clas.virginia.edu/mtstatic/rossetti/design/report. 


\section{BIBLIOGRAPHY}

Aarseth, Espen. Cybertext: Perspectives on Ergodic Literature. Johns Hopkins UP: 1997.

Ades, Dawn. Photomontage. New York: Pantheon, 1976.

Allen, J.F. “Time and Time Again: The Many Ways to Represent Time.” International Journal of Intelligent Systems, vol. 6, no. 4 (July 1991), pp. 341-355.

Bacon. De Augmentis Scientiarum. Of the Advancement and Proficience of Learning; or, The Partitions of Sciences. (Gilbert Wats, trans.) Oxford, Lichfield: 1640.

Barringer, H. et al. The Imperative Future: Principles of Executable Temporal Logic. Research Studies Press Ltd. and John Wiley and Sons: 1996.

Barwise, J. and J. Etchemendy. "Information, Infons, and Inference," in Cooper, Mukai, and Perry (eds), Situation Theory and its Applications, vol. 1, Stanford: CSLI Publications, 1989.

Barwise, J. and A. Shimojima. "Surrogate Reasoning." Cognitive Studies: Bulletin of the Japanese Cognitive Science Society, 4(2): 1995. (7-27).

Bashow, Ira. Temporal Modelling Seminar Presentation, University of Virginia, June 
2001.

Battcock, Gregory. Minimal Art: A Critical Anthology. EP Dutton: 1968.

Beaugrande, Robert de. "Writing and Meaning: Contexts of Research.” Writing in Real Time: Modelling Production Processes. Ed. A. Matsuhashi. Norwood, NJ: Ablex, 1987.

Benford, S., et al. “Networked Virtual Reality and Cooperative Work.” Presence 4.4 (1995): 364-85.

—. "User Embodiment in Collaborative Virtual Environments.” Proceedings of CHI'95. Denver, Colorado: ACM Press, 1995. 242-48.

Benford, S., and D. Snowdon. "Populated Information Terrains: First Steps.” Virtual

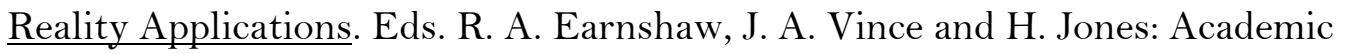
Press, 1995. 27-39.

Bense, Max. "The Projects of Generative Aesthetics." Computers in Art. Ed. Jasia Reichardt. London: Studio Vista, 1971.

Bexte and Künzel, Allwissen und Absturz: der Ursprung des Computers. Insel Verlag, Frankfurt am Main: 1993.

Bickhard, M. H. “Representational Content in Humans and Machines.” Journal of Experimental and Theoretical Artificial Intelligence, 5, pp. 285-333.

Bickle, J. Psychoneural Reduction: The New Wave. MIT Press, Cambridge: 1998. 
Boden, Margaret A. Minds and Mechanisms: Philosophical Psychology and Computational Models. Itaca, N.Y.: Cornell University Press, 1981.

—. The Creative Mind: Myths \& Mechanisms. New York, N.Y.: Basic Books, 1991.

Bonner, Anthony. "Llull's Thought." in Selected Works of Ramon Llull (1232-1316). (trans. Bonner). vol. 1. Princeton UP: 1985.

—. "What Was Llull Up To?" Instituto Brasiliero de Filosofia e Ciencia Raimundo Lulio (available: http://www.geocities.com/Athens/Forum/5284/compbon.html).

Bronstein, H. "Time Schemes, Order, and Chaos: Periodization and Ideology"” in Time. Order. Chaos: The Study of Time IX. Ed. J.T. Fraser. International Universities Press: Madison, CT., 1998.

Burg, J. et al . "Using Constraint Logic Programming to Analyze the Chronology in 'A Rose for Emily"' Computers and the Humanities 34 (4):377-392, December 2000.

Cameron, Charles. "Hipbone Games." (available: http://home.earthlink.net/ hipbone/).

Chalmers, Matthew. "Informatics, Architecture, and Language." Social Navigation in Information Space. Eds. A. Munro, K. Hook and D. Benyon: Springer, 1999.

—. "Structuralist Informatics: Challenging Positivism in Information Systems." Conference of the UK Academy for Information Systems, 1999. 13-22.

- Hermeneutics and Information Representation. Draft. 2001. PDF. Available: 
www.dcs.gla.ac.uk/ matthew/papers/hermeneutics.pdf2002.

Chandrasekaran, B. "Diagrammatic Representation and Reasoning: Some Distinctions," AAAI Symposium Series on Diagrammatic Reasoning: Boston: 1997.

Christensen, W.D. and C.A. Hooker. "Representation and the Meaning of Life." in Clapin, et al. Proceedings of the International Conference on Representation in Mind: New Theories of Mental Representation, 27-29th June 2000, University of Sydney.

Clapin, H., Staines, P. \& Slezak, P. Proceedings of the International Conference on Representation in Mind: New Theories of Mental Representation, 27-29th June 2000, University of Sydney. To be published by Elsevier.

Corelli, Marie. The Soul of Lilith, Richard Bentley and Son: London, 1892.

Coyne, Richard. Designing Information Technology in the Postmodern Age: from Method to Metaphor. Cambridge, Mass.: MIT Press, 1995.

Cramer, Florian. "On Literature and Systems Theory." (8 April 2001). Available: http://userpage.fu-berlin.de/ cantsin/homepage/\#theory

Cramer Florian and Ulrike Gabriel. "Software Art." (15 August 2001). Available: http://userpage.fu-berlin.de/ cantsin/homepage/

Crawford, Chris. The Art of Computer Game Design. Macmillan/McGraw-Hill: 1984. (available: http://www.vancouver.wsu.edu/fac/peabody/gamebook/Coverpage.html) 
Cross, Nigel. "Designerly Ways of Knowing." Design Studies 3.4 (1984): 221-27.

Dalton, O. M. "A Portable Dial in the Form of a Book, with Figures Derived from Ramon Lul.” Archaeologia vol. 74. Oxford (1925). 89-102.

Davis, E. Representations of Commonsense Knowledge. San Mateo: Morgan Kaufmann Publishers, 1990.

Deray, K. “Avatars: A Shifting Interaction.” Selected Papers from the 2001 Pan-Sydney Workshop on Visual Information Processing. Sydney, Australia: Conferences in Research and Practice in Information Technology, 2002. Vol. 11.

Johanna Drucker. "Digital Ontologies: The Ideality of Form in/and Code Storage -orCan Graphesis Challenge Mathesis?” in Leonardo. 34.2 (2001): 141-145.

—. Ivanhoe game development sketch (JPG format, 2001). Available: http://www.speculativecomputing.org/ivanhoe/design.html

—. "Theory as Praxis: The Poetics of Electronic Textuality." Modernism / Modernity 9.4 (2002): 683-691.

—. Ivanhoe interface proposal (PowerPoint format, 2003). Available: http://www.speculativecomputing.org/ivanhoe/design.html

—. email: "Re: time mod inflections." (Personal communication to the author). October 2003. 
Drucker, Johanna, and Bethany Nowviskie. "Temporal Modelling: Visualizing Temporal Relations for Humanities Scholarship (Part One: Literature Review and Conceptualization).” IDJ: Information Design Journal (forthcoming). 2003 draft available: http://www.iath.virginia.edu/time/.

Drucker, Johanna, with Bethany Nowviskie. "Speculative Computing: Aesthetic Provocations in Humanities Computing.” A Companion to Digital Humanities. Eds. John Unsworth, Ray Siemens and Susan Schreibman. Oxford: Blackwell Publishers. (forthcoming 2004).

Elrad, Tzilla, et al. "Aspect-Oriented Programming: Introduction." in $\underline{\text { Communications }}$ of the ACM 44:10 (2001): 28-32.

Elster, Jon. “Conventions, Creativity, and Originality.” Rules and Conventions : Literature, Philosophy, Social Theory. Ed. Mette Hjort. Baltimore, Md.: John Hopkins University Press, 1992.

—. "Fullness and Parsimony." Explanation and Value in the Arts. Eds. Ivan Gaskell and Salim Kemal. New York: Cambridge University Press, 1993.

Euler, L. Lettres à une Princesse d'Allemagne. St Petersburg: L’Academie Imperiale des Sciences, 1768 .

Flanagan, Mary. “Mobile Identities, Digital Stars, and Post Cinematic Selves.” Wide Angle 21.1 (1999): 77-93.

Foster, K. I . "Lexical Processing.” Language: An Invitation to Cognitive Science. Eds. D. N. Osherson and H. Lasnik. Vol. 1. Cambridge: MIT Press, 1990. 95-131. 
Fraser, J.T. Time, The Familiar Stranger. Massachusetts UP, 1987.

Fry, Benjamin. Valence. (available: http://acg.media.mit.edu/people/fry/valence/).

Gardner, M. "The Ars Magna of Ramon Lull." Logic Machines and Diagrams. Chicago UP, 1982.

Glasgow, J.I. and D. Papadias. "Computational Imagery." Cognitive Science 3: 1992. (355$394)$.

Goel, V. Sketches of Thought. Cambridge: MIT Press, 1995.

Hammer, E. "Reasoning with Sentences and Diagrams.” Notre Dame Journal of Formal Logic 35(1): 1995. (73-87).

Harrison, William, and Harold Ossher. "Subject-Oriented Programming: A Critique of Pure Objects.” Proceedings of the Eighth Annual Conference on ObjectOriented Programming Systems, Languages, and Applications. Washington, DC, 1993.

Harrison, William, et al. "Subjectivity in Object-Oriented Systems Workshop Summary.” Proceedings of the Ninth Annual Conference on Object-Oriented Programming Systems, Languages, and Applications. Portland, Oregon, 1994. Addendum.

Hesse, Hermann. The Glass Bead Game (Magister Ludi). trans. Winston, R. and C. Picador USA: 2002. 
Iwasaki, Y. “Session Report.” NSF Workshop on Visual Cognition and DecisionMaking in the Spatial Domain, May 15-17, 1997.

James, William. Pragmatism: A New Name for Some Old Ways of Thinking. New York: 1909. (52-61).

Jarry, Alfred. Exploits and Opinions of Doctor Faustroll, 'Pataphysician, Book II, Chapter 8 (1907) tr. Simon Watson-Taylor.

Jensen, C. S., et al. “A Glossary of Temporal Database Concepts.” Proceedings of ACM SIGMOD International Conference on Management of Data 23, 1 (March, 1994).

Jonker, C. et al. “A Temporal Modelling Environment for Internally Grounded Beliefs, Desires, and Intentions.” Cognitive Systems Research Journal, vol. 4 (3), 2003, pp. 191-210.

Jordan, P.W. Determining the Temporal Ordering of Events in Discourse. Unpublished masters thesis for Carnegie Mellon Computational Linguistics Program, 1994.

Kirschenbaum, M. (ed.) “Special Issue: Image-Based Humanities Computing.” Computers and the Humanities 36.1 (2002).

—. (moderator). "What is Text?" at ACH/ALLC 1999. Participants: McGann and Renear. others? in proceedings?

Kristensen, Bent Bruun. "Subjective Method Interpretation in Object-Oriented 
Modelling." Proceedings of the 5th International Conference on Object-Oriented Information Systems. Paris, France, 1998.

Künzel, The Birth of the Machine: Raymundus Lullus and His Invention.

Lagus, Krista. Automated pagination of the generalized newspaper using simulated annealing. Master's thesis, Helsinki University of Technology. Otaniemi, Finland: 1995.

Leibniz, Dissertatio de Arte Combinatoria. Leipzig, 1663.

Lemon, O., de Rijke, M. and Shimojima, A. "Efficacy of Diagrammatic Reasoning." Journal of Logic, Language, and Information 8(3): 1999. (265-271).

Leyton, Michael. Symmetry, Causality, Mind. MIT Press: 1999.

—. Process Grammar. (online). 4 HTML pages beginning at: http://www.rci.rutgers.edu/ mleyton/freeform 1.htm

Llull, Ramon. Selected Works of Ramon Llull (1232-1318). trans. Anthony Bonner. (2 vols.). Princeton UP: 1985.

Lovelace (Countess of), Augusta Ada Byron King (translator). "Sketch of the Analytical Engine invented by Charles Babbage, Esq. By L. F. MENABREA, of Turin, Officer of the Military Engineers.” Scientific Memoirs 3 (1843), 666-731.

Luca, Gerashim, and Dolfi Trost. Dialectique de dialectique. Message addressé au mouvement surréaliste international. Bucharest, 1945. 
MacEachren, Alan. How Maps Work. New York: Guilford Press, 1995.

Mateas, M. "Expressive AI: A Hybrid Art and Science Practice.” Leonardo: Journal of the International Society for Arts, Sciences, and Technology 34.2 (2001): 147-53.

—. "Not Your Grandmother's Game: AI-Based Art and Entertainment." Proceedings of the 1999 AAAI Spring Symposium on Artificial Intelligence and Computer Games. Menlo Park: AAAI Press, 1999. 64-68. Vol. SS-99-02.

Maturana, Humberto R., and Francisco J. Varela. uutopoiesis and Cognition: The Realization of the Living. Dordrecht, Holland; Boston: Reidel Publishing, 1980.

McGann, Jerome. Dante Gabriel Rossetti and the Game That Must Be Lost. Yale UP: 2000.

—. "Texts in N-Dimensions and Interpretation in a New Key." Unpublished Paper.

—. "Imagining What You Don't Know: the Theoretical Goals of the Rossetti Archive." (available: http://www.iath.virginia.edu/ jjmef/chum.html).

—. Radiant Textuality. Palgrave/St. Martin's Press: 2001.

McGann, Jerome, and Lisa Samuels. “Deformance and Interpretation.” New Literary History 30.1 (1999): 25-56.

Miller, John David and John Maeda. “A Stitch in Time: Visualizing History Through Unit Forms and Repetition Structires.” (unpublished submission to CHI98.) 
Molyneux, Peter, Dene Carter, and Simon Carter. Project Ego: Big Blue Box. 2003. Available: http://www.bigbluebox.com.

Motherwell, Robert. The Dada Painters and Poets: An Anthology. (reprint) Belknap Press, 1989.

Nowviskie, Bethany. Future Ivanhoe Visualizations: Evolving Avatar. 2002. SpecLab. Available: http://www.speculativecomputing.org/ivanhoe/design/demo/.

—. "Ivanhoe and Game Design." 2001. SpecLab; presented at Virginia Humanities and Technology Association Conference 2000. Available: http://www.speculativecomputing.org/ivanhoe/framework/design.html.

—. "Temporal Modelling: Visualizing Temporal Relations for Humanities Scholarship (Part Two: Composition Tool Design).” IDJ: Information Design Journal (forthcoming). 2003 draft available: http://www.iath.virginia.edu/time/.

—. Temporal Modelling: Update 2003. SpecLab. Available: http://www.iath.virginia.edu/time.

O’Brian, Patrick. H.M.S. Surprise, New York: W.W. Norton \& Company, 1991.

O’Toole, M.A. “The Theory of Serialism in The Third Policeman.” Irish University Review. 18(2):215-225. 1988.

Osborne, Randy. "Extending the Multilisp Sponsor Model." Proceedings of the 1992 Parallel Symbolic Computing Workshop at MIT in Springer-Verlag Lecture Notes on Computer Science, November 1993. 
Paley, W. B. TextArc. (available: http://www.textarc.org).

Peckham, Morse. "Historiography and The Ring and the Book." Victorian Poetry. (6:3-4). 1968. (243-258).

Peirce, C.S. Collected Papers. Cambridge, MA: Harvard UP, 1933.

Penny, Simon, Jeffrey Smith, and Phoebe Sengers. "Traces: Embodied Immersive Interaction with Semi Autonomous Avatars." Convergence: The Journal of $\underline{\text { Research into New Media Technologies }} 7.2$ (2001).

Marjorie Perloff, Radical Artifice: Writing Poetry in the Age of Media (Chicago and London: University of Chicago Press, 1992

Pettifer, Steve, and Adrian West. "Subjectivity and the Relaxing of Synchronization in Networked Virtual Environments.” Proceedings of the ACM Symposium on Virtual Reality Software and Technology. London: ACM Press, 1999.

Port, R. F. and Gelder, T. van (eds.) Mind as Motion: Explorations in the Dynamics of Cognition. MIT Press, 2001.

Price, H. “The View from Nowhen” in Time's Arrow and Archimedes' Point. Oxford UP: New York, 1996.

Queneau, Raymond. Entretiens avec Georges Charbonnier. Paris: Gallimard, 1962.

—. Exercises de Style. Paris: Gallimard, 1979. 
Ramsay, Stephen J. Algorithmic Criticism. Dissertation, University of Virginia, 2003.

Ray, Benjamin. Salem Witch Trials Project. Institute for Advanced Technology in the Humanities (IATH). (available: http://www.iath.virginia.edu/salem).

Reynolds, Teri. "Spacetime and Imagetext." Germanic Review. 73(2):161-74. 1998 Spring.

Roubaud, J. "Mathematics in the Method of Raymond Queneau. in Motte, W. (ed). OuLiPo: A Primer of Potential Literature. Lincoln: Nebraska UP, 1986. (79-96).

Russell, Bertrand. "Vagueness" (1923). in J. Slater, ed., Essays on Language, Mind, and Matter 1919-26 (The Collected Papers of Bertrand Russell). London: Unwin Hyman. (145-154).

Sales, Ton. "Llull as Computer Scientist, or Why Llull Was One of Us." Instituto Brasiliero de Filosofia e Ciencia Raimundo Lulio. (available: http://www.geocities.com/llull_brazil/compsale.html).

Sandor, O., C. Bogdan, and J. Bowers. "Aether: An Awareness Engine for CSCW.” Proceedings of the Fifth European Conference on Computer Supported Cooperative Work. Eds. H. Hughes and Prinz. W. Lancaster, UK: Kluwer, 1997. 221-36.

Sansing, Chandler. "Case Study and Appeal: Building the Ivanhoe Game for Classroom Flexibility" (forthcoming in Text Technology).

Scaramalia, Marlene, and Carl Bereiter. "Literate Expertise." Toward a General Theory 
of Expertise: Prospects and Limits. Eds. K. Anders Ericsson and Jacqui Smith. New York: Cambridge University Press, 1991.

Schön, D. "Designing as a Reflective Conversation with the Materials of a Design Situation." Research in Engineering Design 3 (1992): 131-47.

Schreiber, F.A. "Is Time a Real Time? An Overview of Time Ontology in Informatics" in Real Time Computing, 1992.

Sengers, Phoebe, Simon Penny, and Jeffrey Smith. Traces: Semi-Autonomous Avatars: Paper E0169: Center for Virtual Environments, University of Salford, 2000.

Shaw, W. David. "Edward Burne-Jones and Pre-Raphaelite Melancholy.” University of Toronto Quarterly, 66:2 (Spring 1997).

Shilling, John, and Peter Sweeney. "Three Steps to Views: Extending the ObjectOriented Paradigm.” Proceedings of the Conference on Object-Oriented Programming, Systems, and Languages, and Applications. New Orleans: ACM Press, 1989. 353-61.

Shimojima, A. On the Efficacy of Representation. Ph.D. Thesis, Indiana University, 1996.

Shin, Sun-Yoo. The Logical Status of Diagrams. Cambridge UP, 1994.

Shin, Sun-Yoo, and Oliver Lemon. “Diagrams.” Stanford Encyclopedia of Philosophy (online).

Shubik, Martin. "On the Scope of Gaming." Management Science 18.5 (1972): 20-36. 
Sinclair, S. HyperPo: Text Analysis and Exploration Software. (available: http://qsilver.queensu.ca/QI/HyperPo/).

Smith, Gareth. "Cooperative Virtual Environments: Lessons from 2D Multi User Interfaces.” Proceedings of the 1996 ACM Conference on Computer Supported Cooperative Work. Boston, Mass.: ACM Press, 1996. 390-98.

Snodgrass, Adrian, and Richard Coyne. "Is Designing Hermeneutical?" Architectural Theory Review 1.1 (1997): 65-97.

—. "Models, Metaphors, and the Hermeneutics of Designing." Design Issues 9.1 (1992): $56-74$.

Snowdon, Dave, and Kai-Mikael Jää-Aro. "How Not to Be Objective.” Collaborative Virtual Environments: Digital Places and Spaces for Interaction. Eds. Elizabeth F. Churchill, Alan J. Munro and David N. Snowdon. New York: SpringerVerlag, 2001. 143-59.

—. "A Subjective Virtual Environment for Collaborative Information Visualization." Proceedings of Virtual Reality Universe '97. Santa Clara, California, 1997.

Speculative Computing Laboratory. SpecLab materials online (design: Nowviskie; various authors). Available: http://www.speculativecomputing.org/

Spence, Robert. “A Framework for Navigation.” International Journal of HumanComputer Studies 51.4 (1999): 919-45. 
Sperberg-McQueen, C. M. "What Matters?” (Extreme Markup 2002) Available: http://www.w3.org/People/cmsmcq/2002/whatmatters.html.

Steedman, M. “The Productions of Time.” (draft tutorial notes 2.0: University of Edinburgh.) Available: ftp://ftp.cis.upenn.edu/pub/steedman/temporality/.

Stefik, Mark, et al. "Beyond the Chalkboard: Computer Support for Collaboration and Problem Solving in Meetings.” Communications of the ACM 30.1 (1987): 32-47.

Suber, Peter. The Paradox of Self-Amendment. Peter Lang Publishing, 1990.

Swift, J. Gulliver's Travels. (Rivero, A. J. ed.) W.W. Norton \& Co: 2001.

Symes, Colin. "Writing by Numbers: Oulipo and the Creativity of Constraints." Mosaic 32.3 (1999): 87-107.

Tufte, Edward. Envisioning Information. Cheshire, CT: Graphics Press: 1990.

Turing, Alan. “Computing Machines and Intelligence," in MIND, A Quarterly Review of Psychology and Philosophy, Vol. 59, No. 236, October, 1950, pp. 433-460.

Unsworth, John. “The Importance of Failure.” Journal of Electronic Publishing. 3(2): 1997. (available: http://www.press.umich.edu/jep/o3-02/unsworth.html).

—. "Tool Time: or, 'Haven't We Been Here Already?"' Transforming Disciplines: The Humanities and Computer Science. NINCH conference: 18 January 2003: Washington, DC. (available: http://www.iath.virginia.edu/ jmu2m/carnegieninch.03.html). 
Venn, J. Symbolic Logic. London: Macmillan, 1881.

Wickens, C. D. “Frames of Reference for Navigation.” Attention and Performance. Eds. D. Gopher and A. Koriat. Vol. 17. Cambridge, Mass: MIT Press, 1999. 113-44.

Winograd, Terry, and Carlos F. Flores. Understanding Computers and Cognition: A New Foundation for Design. Language and Being. Norwood, N.J.: Ablex Pub. Corp., 1986.

Winograd, Terry, ed. Bringing Design to Software. Addison-Wesley, 1996.

—. "From Programming Environments to Environments for Designing." Communications of the ACM 38.6 (1995): 65-74.

Yang, Huahai, and Gary Olson. "Exploring Collaborative Navigation: The Effect of Perspectives on Group Performance.” Proceedings of the 4th International Conference on Collaborative Virtual Environments. Bonn, Germany: ACM Press, 2002. 135-42.

Yates, Frances. The Art of Memory. Routledge: 1999.

Zweig, Janet. "Ars Combinatoria: Mystical Systems, Procedural Art, and the Computer." Art Journal 56.3 (1997): 20-29. 